\title{
Regulation of clade I TGA transcription factors of Arabidopsis thaliana during salicylic acid-mediated defense response
}

\author{
Dissertation \\ for the award of the degree \\ “Doctor rerum naturalium" \\ of the Georg-August-Universität Göttingen
}

within the doctoral program IRTG 2172 PRoTECT

of the Georg-August University School of Science (GAUSS)

\author{
submitted by \\ Jelena Budimir \\ from Sinj, Croatia
}

Göttingen 2020 


\section{Thesis Advisory Committee}

Prof. Dr. Christiane Gatz, Department for Plant Molecular Biology and Physiology, Albrechtvon-Haller-Institut, Georg-August-Universität Göttingen

Prof. Dr. Petr Karlovsky, Department of Crop Sciences, Molecular Phytopathology and Mycotoxin Research, Georg-August-Universität Göttingen

Prof. Dr. Xin Li, Department of Botany, University of British Columbia

\section{Members of the Examination Board}

$1^{\text {st }}$ Reviewer

Prof. Dr. Christiane Gatz, Department for Plant Molecular Biology and Physiology, Albrechtvon-Haller-Institut, Georg-August-Universität Göttingen

$2^{\text {nd }}$ Reviewer

Prof. Dr. Petr Karlovsky, Department of Crop Sciences, Molecular Phytopathology and Mycotoxin Research, Georg-August-Universität Göttingen

Prof. Dr. Andrea Polle, Department of Forest Botany and Tree Physiology, Georg-AugustUniversität Göttingen

Prof. Dr. Volker Lipka, Department of Plant Cell Biology, Albrecht-von-Haller-Institut, GeorgAugust-Universität Göttingen

PD Dr. Marcel Wiermer, Department of Plant Cell Biology -Molecular Biology of PlantMicrobe Interactions, Albrecht-von-Haller-Institut, Georg-August-Universität Göttingen

Date of oral examination: $12^{\text {th }}$ December 2019 
Za Joshuu, hvala za ljubav. 


\section{Contents}

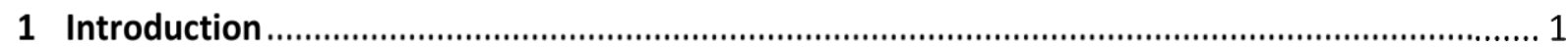

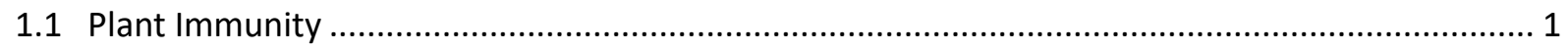

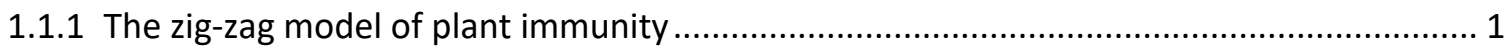

1.1.2 The plant immune response can be activated locally and systemically.............................. 2

1.1.3 The plant immune response is guided by plant defense hormones ................................... 2

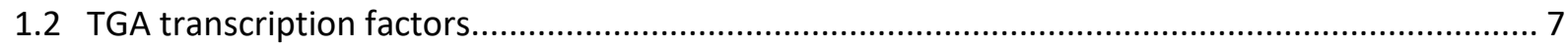

1.2.1 Clade I TGA transcription factors have a broad spectrum of activity ................................. 7

1.2.2 Clade II and III TGA transcription factors are involved in plant immunity .......................... 11

1.2.3 Clade IV and V TGA transcription factors are involved in plant development................... 11

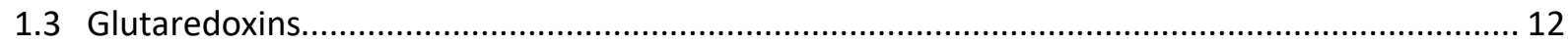

1.3.1 Glutaredoxins in model plant Arabidopsis thaliana ......................................................... 12

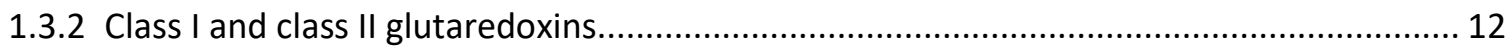

1.3.3 Class III CC-type glutaredoxins interact with TGA transcription factors ............................. 13

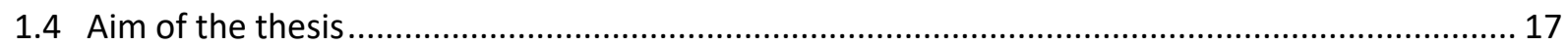

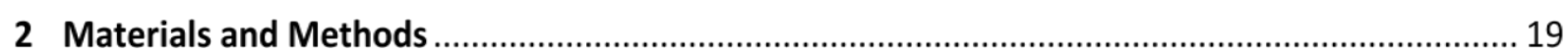

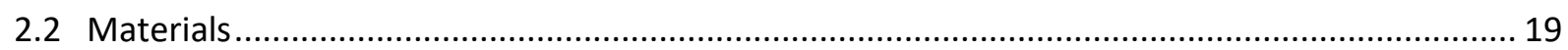

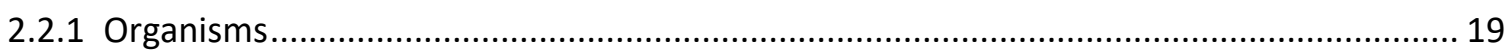

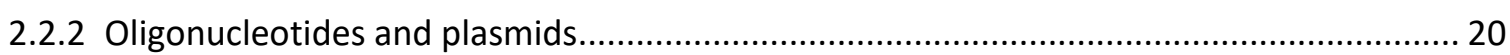

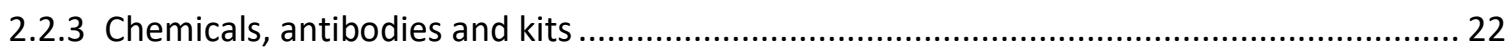

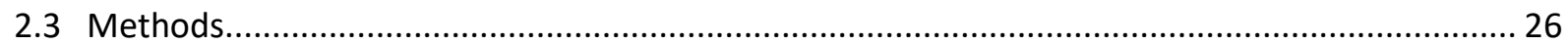

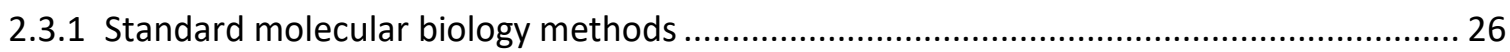

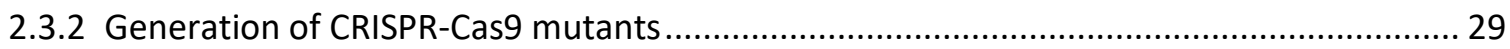

2.3.3 Reporter gene assay in Arabidopsis protoplasts ............................................................ 31

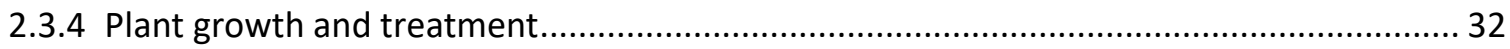

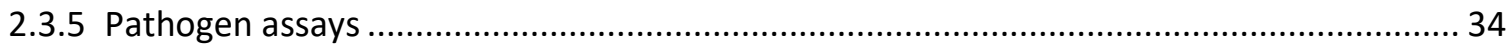

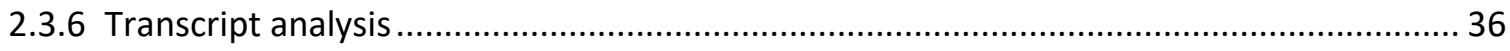

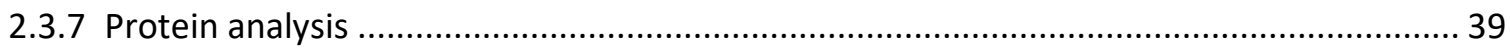

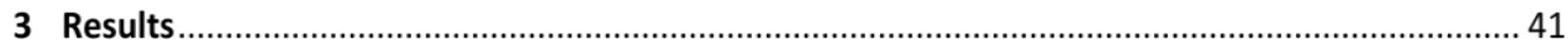

3.1 RNA sequencing data revealed that TGA1/TGA4 activate a subset of genes downstream of SA. 41

3.1.1 SA treatment induced transcriptional changes in sid2 and sid 2 tga1 tga4 mutant ........... 41

3.1.2 A group of 207 SA-inducible genes was TGA1/TGA4-dependent ...................................... 43

3.1.3 Induction of SARD1 is TGA1/TGA4-independent after SA treatment ................................ 46

3.1.4 The promoter regions of SA-induced genes are enriched in TGA-binding sites ................. 48

3.2 The group of TGA1/TGA4-dependent genes belongs to the classical SA signaling pathway........ 51 
3.2.1 TGA1/TGA4-dependent regulation of SA-inducible genes can be observed also in the wildtype background.

3.2.2 Induction of TGA1/TGA4-regulated genes is TGA2/TGA5/TGA6- and NPR1-dependent ... 52

3.2.3 DLO1 is a potential direct target of TGA1/TGA4, TGA2/TGA5/TGA6 and SARD1 53

3.3 The redox state of the four critical cysteine residues of TGA1 is not important for its transcriptional activity.

3.3.1 The redox state of the four critical cysteine residues is not important for induction of TGA1/TGA4-dependent genes downstream of SA.

3.4 Induction of TGA1-regulated genes after pathogen infection is dependent on clade I and II TGA transcription factors and NPR1

3.4.1 TGA1/TGA4 are important for induction of the target genes after infection with hemibiotrophic pathogen Pseudomonas syringae pv. maculicola.

3.4.2 The redox state of the four critical cysteine residues is not important for induction of TGA1/TGA4-dependent genes upon pathogen attack in SAR experiment

3.5 TGA1 protein is predominantly expressed in roots and vascular tissue of Arabidopsis thaliana 60

3.5.1 TGA1 protein is abundant in roots of Arabidopsis thaliana. 60

3.5.2 TGA1 promoter is expressed in vascular tissue and in roots of Arabidopsis thaliana 62

3.5.3 TGA1 does not accumulate in the leaves or the midrib after SA treatment, but does after Pseudomonas syringae infection in the systemic tissue

3.6 Transiently expressed TGA1, TGA2 and SARD1 activate DLO1 promoter in Arabidopsis mesophyll protoplasts

3.6.1 DLO1 expression is reduced in mutants of clade I and clade II TGA transcription factors .66

3.6.2 TGA1 activates DLO1 promoter in the mutant of clade I and clade II TGA transcription factors

3.6.3 TGA1, TGA2 and SARD1 activate DLO1 promoter in the mutant of clade II TGA transcription factors

3.6.4 Mutation in the C-box but not in the A-box element abolishes TGA1- and TGA2-induced DLO1 promoter activity

3.7 Highly expressed CC-type glutaredoxins are not responsible for the low expression of SAinducible genes in tga1 tga4 mutant.

3.7.1 Expression of glutaredoxin-like proteins ROXY11, ROXY12, ROXY13, ROXY14 and ROXY15 was increased in sid2 tga1 tga4 mutant

3.7.2 CRISPR-Cas9 was used to knock out a gene cluster

3.7.3 Elevated ROXY11-15 levels in tga1 tga4 mutant do not repress TGA1/TGA4 dependent genes after SA treatment.

3.7.4 Elevated ROXY11-15 levels in tga1 tga4 mutant are not important for induction of TGA1/TGA4-dependent genes upon pathogen attack in SAR experiment

3.7.5 Elevated ROXY11-15 levels in tga1 tga4 mutant are not important for susceptibility of tga1 tga4 mutant

3.8 ROXY9-modulated repression of TGA1 is not released in roxy6 roxy7 roxy8 roxy9 mutant 
3.8.1 Overexpression of ROXY9 mimics tga1 tga4 mutant after SA treatment.......................... 78

3.8.2 Using CRISPR-Cas9 to knock-out ROXY6 and ROXY7 in roxy9 mutant background ............ 78

3.8.3 ROXY9-mediated repression of TGA1 is not released in roxy6 roxy7 roxy8 roxy9 mutant

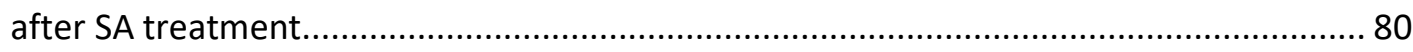

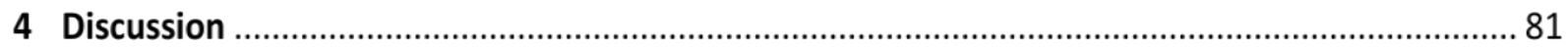

4.2 TGA1 is modulated by SA but what is its role downstream of SA? .......................................... 82

4.2.1 Identification of TGA1/TGA4-dependent genes downstream of SA ................................... 82

4.2.2 TGA1/TGA4-dependent genes are inducible through NPR1-TGA2/TGA5/TGA6 and

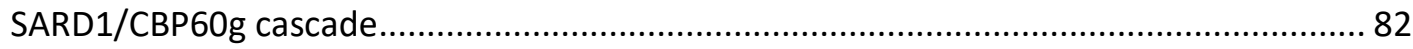

4.2.3 TGA1, TGA2 and SARD1 activate DLO1 promoter in Arabidopsis mesophyll protoplasts and this is dependent on TGACGTCA motif in DLO1 promoter ................................................ 84

4.3 Are critical cysteine residues important for TGA1/TGA4 role downstream of SA? ..................... 87

4.3.1 Critical cysteine residues are not important for induction of TGA1/TGA4-dependent genes downstream of salicylic acid

4.3.2 The critical cysteine residues are not important for induction of TGA1/TGA4-dependent genes after Pseudomonas syringae infection

4.3.3 Induction of TGA1/TGA4-dependent genes after pathogen infection depends on NPR1TGA2/TGA5/TGA6 and SARD1/CBP60g.

4.4 Do highly expressed glutaredoxins in tga1 tga4 mutant suppress the activity of TGA2/TGA5/TGA6?

4.4.1 ROXY11-15 are not repressors of TGA2/TGA5/TGA6 in tga1 tga4 mutant

4.5 Because TGA1 is repressed by ectopically expressed ROXY9, does ROXY9 loss-of-function have a reverse effect?

4.5.1 ROXY9-mediated repression of TGA1/TGA4 is not released after SA treatment 91

5 Summary 93

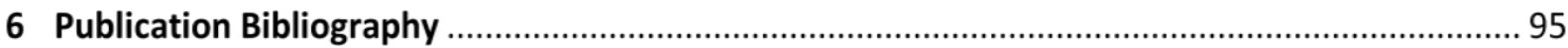

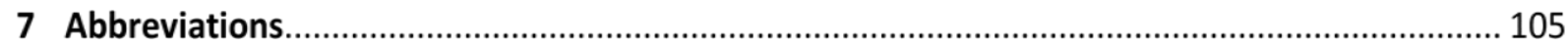

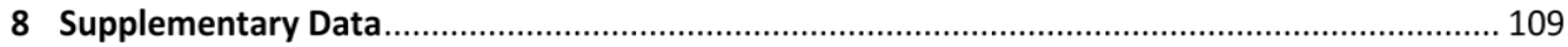

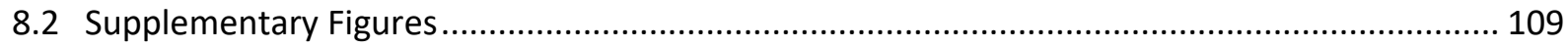

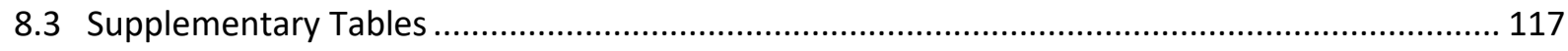

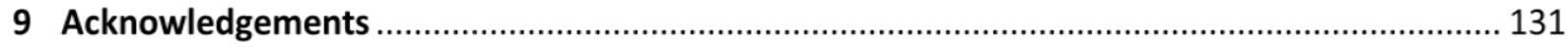




\section{Introduction}

\subsection{Plant Immunity}

Plants are generally resistant to pathogens, partly due to the presence of two physical barriers at the surface, the first being the cuticle and the second the cell wall. However, a handful of fungal pathogens can penetrate these layers using a combination of mechanical rupture and enzymatic degradation (Yeats and Rose 2013). Others, such as bacteria, use natural openings, most importantly stomata, to enter the plant (Katagiri et al. 2002). Once it the plant, a very sophisticated immune system is ready for the invaders.

\subsubsection{The zig-zag model of plant immunity}

The plant immune response, often described by the zig-zag model, consists of four levels, that have evolved during the evolutionary competition between hosts and pathogens (Jones and Dangl 2006). They describe the robustness of the immune response against the pathogen and the subsequent suppression of the immune response by the pathogen.

The first level is characterized by host's recognition of molecules which are derived from the pathogen propagating in the intercellular space. Perception of the so-called microbe- and pathogen associated molecular patterns (MAMPs and PAMPs) takes place at the plasma membrane, which harbors pattern recognition receptors (PRR). Alerted PRRs initiate signaling cascades leading to the activation of the first layer of plant defense response, PAMP triggered immunity (PTI). Physiological changes caused by PTI restrain the growth of the pathogen.

At the second level, successful pathogens have evolved specific types of effector molecules to counteract PTI. Pathogens inject effector molecules in the plant using the bacterial type 3 secretion system (T3SS) (Alfano and Collmer 2004). After injection, effectors modulate plant defense responses to support propagation and survival of the pathogen. In the host plant, this leads to effector triggered susceptibility (ETS) (Hauck et al. 2003).

At the third level, effector molecules are recognized by the intracellular plant immune receptors, formerly called RESITANCE (R) proteins. Recognition of specific pathogen effectors 


\section{Introduction}

leads to the activation of the second layer of the plant immune response, effector triggered immunity (ETI). Similar as PTI, ETI leads to restriction of pathogen growth.

The fourth level describes a long time period during which effector and resistance genes of pathogens and host plants, respectively, evolved due to the strong selective pressure. The pathogens improved their effector collection through either (i) evolution of new and changed effectors or (ii) elimination of the ones which can be recognized by the host plant. Meanwhile, plants evolved novel RESISTANCE $(R)$ genes, that can recognize new or changed effectors and activate ETI. The evolution and selection of effector and resistance genes is interdependent and often referred to as gene-for-gene hypothesis (Flor, 1975).

\subsubsection{The plant immune response can be activated locally and systemically}

The two layers of plant immunity, PTI and ETI, are important for the activation of defense responses at the site of infection. Upon activation, plants go through physiological changes such as generation of reactive oxygen species (ROS), production and secretion of antimicrobial compounds, calcium influx, biosynthesis of defense hormones and activation of downstream immune responses. Locally, these changes limit the propagation of pathogen (Bigeard et al. 2015).

Along with local immunity, plants have evolved a broad spectrum and long-lasting immune strategy to combat pathogens in the unchallenged tissue. This part of the immune response is called systemic acquired resistance (SAR) (Durrant and Dong 2004). Certain types of immune molecules, which accumulate at the site of infection, can be transported via the phloem to the uninfected distal tissue. In this tissue, sensing of the so-called mobile signals triggers a set of biochemical, molecular and physiological changes. This phenomenon is known as priming and it enables fast and robust reaction to the secondary pathogen attack.

\subsubsection{The plant immune response is guided by plant defense hormones}

Plants have multiple defense hormones that modulate immune responses against different types of pathogens. The most important defense hormones are salicylic acid (SA), jasmonic acid (JA) and ethylene (ET). 


\section{Introduction}

Dependent on their feeding strategy, pathogens can be biotrophs, necrotrophs or hemibiotrophs. As their name suggests, biotrophs thrive on the living host and necrotrophs eliminate the host and feed on its cell content. Hemi-biotrophs exert both lifestyles depending on the stage of their life cycle (Glazebrook 2005). Defense against biotrophic and hemibiotrophic pathogens is coordinated by SA. JA and ET are important for defense responses against necrotropic pathogens (Beckers and Spoel 2006).

Activation of defense mechanisms is extremely costly for the plant and results in growth defects, often observed in autoimmunity mutants (van Wersch et al. 2016). Therefore, biosynthesis of defense hormones is tightly controlled and involves a complex regulatory machinery. In this thesis, we were investigating processes specific for the SA signaling pathway in model plant Arabidopsis thaliana.

\subsubsection{SA biosynthesis is transcriptionally regulated after a pathogen infection}

SA is biosynthesized either via the PAL (PHENYALANIN AMMONIA-LYASE) or the ICS (ISOCHORISMATE SYNTHASE) pathway. The latter pathway is the major contributor to SA production after infections (Dempsey et al. 2011). Although it has been researched for more than two decades, the ICS pathway was only recently elucidated. The first step of SA biosynthesis takes place in plastids and involves the enzyme ICS1 which generates isochorismate (IC) from chorismic acid (CA). Transport of IC from plastid to cytosol is executed by the EDS5 (ENDANCED DISEASE SUSCEPTIBILITY5) transporter (Nawrath et al. 2002; Rekhter et al. 2019). In the cytosol, IC is converted to IC-9-gutamate by amidotransferase PBS3 (avrPphB SUSCEPTIBLE3) (Rekhter et al. 2019). Finally, IC-9-glutamate spontaneously decomposes to SA and the glutamate byproduct in the cytosol.

Transcriptional regulation of the genes encoding for the enzymes of the ICS pathway is guided through calcium signaling (Seyfferth and Tsuda, 2014). Members of a plant-specific group of transcription factors, CBP60g (CAMODULIN-BINDING PROTEIN 60-LIKE g) and SARD1 (SYSTEMIC ACQUIRED RESISTANCE DEFICIENT1) are the most important activators of ICS1 and PBS3 (Zhang et al. 2010; Sun et al. 2015). However, in the uninduced state, CBP60g and SARD1 are negatively regulated by calcium-sensitive repressors CAMTA1/CAMTA2/CAMTA3 (CALMODULIN-BINDING TRANSCRIPTION FACTOR 1/2/3) (Kim et al. 2020; Sun et al. 2020). The 


\section{Introduction}

repression is released upon pathogen infection due to the increase of intracellular calcium concentration. The surge of calcium levels is sensed by calcium receptors such as calmodulin (CaM) (Seyfferth and Tsuda, 2014). When activated, CaM binds CAMTA1/CAMTA2/CAMTA3 proteins and activates their auto-repressive function, hence releasing the repression of SA biosynthesis (Kim et al. 2017). Transcription of SARD1 and CBP60g is activated by transcription factor TGA1 (TGACG-BINDING FACTOR1) (Sun et al. 2018). In addition to the repression of CAMTA1/CAMTA2/CAMTA3 proteins, CaM activates the CBP60g protein. Therefore, CBP60g is essential for the induction of SA biosynthesis genes in the early phase, while SARD1 is more important in the late phase of infection (Wang et al. 2009, Wang et al. 2011).

\subsubsection{Signaling downstream of SA is modulated through NPR1}

Downstream of SA biosynthesis, NPR1 (NON-EXPRESSOR OF PR GENES1) is an essential regulator of SA-induced transcriptional reprograming. NPR1 is a founding member of a small gene family in Arabidopsis thaliana, consisting of six NPR1-like genes. The main characteristics of all members are two protein-protein interaction domains. The first is a BTB/POZ (for BroadComplex, Tramtrack, and Bric-a-Brac/POX virus and Zinc finger) domain and the second is a series of four ankyrin repeats. While NPR1 and its closest homologues NPR3/NPR4 are characterized with respect to their involvement in plant immunity, the other two members BOP1/BOP2 (BLADE-ON-PETIOLE1/2) are critical for flower development (Hepworth et al. 2005). Although they are all involved in gene regulation, they lack a DNA-binding domain and were ruled out as transcription factors.

NPR1 loss-of-function mutants were identified independently by several groups while screening for mutants which were deficient in chemically induced SAR and/or SA-induced transcriptional changes (Cao et al. 1994; Delaney et al. 1995; Jyoti Shah et al. 1997). The mutant accumulated wild-type-like SA levels but was unable to induce classical SA-responsive genes such as PR1 (PATHOGENESIS RELATED1) and BGL2 (6-1,3-GLUCANASE2). Therefore, the role of the protein was placed downstream of SA biosynthesis.

\subsection{NPR1 protein is sensitive to redox changes in the cytosol}

As the master regulator of SA signaling, the NPR1 protein is tightly regulated. In the uninduced state, it is retained in the cytosol through oligomerization by intermolecular disulfide bridges 


\section{Introduction}

(Kinkema et al. 2000; Mou et al. 2003). After pathogen attack, the cytosolic environment shifts to the more reducing conditions. This leads to the activation of thioredoxins TRX3 and TRX5, which in turn reduce the intermolecular disulfide bridges of NPR1. When reduced, NPR1 is monomerized and transported to the nucleus where it induces $P R$ gene transcription (Kinkema et al. 2000; Mou et al. 2003; Tada et al. 2008). In addition to these regulatory events at the protein level, transcription of the NPR1 gene is induced by SA treatment and pathogen challenge.

\subsection{NPR1-activated gene transcription is regulated by SA and NPR3/NPR4}

Currently, there are two models explaining NPR1-induced gene regulation. The first model proposes regulation of NPR1 at the protein level by the high- and low-affinity SA receptors, NPR4 and NPR3, respectively (Spoel et al. 2009; Fu et al. 2012). In the uninduced state, the nuclear fraction of NPR1 is targeted for proteasomal degradation by NPR4 (Fu et al. 2012). Pathogen infection causes increase of SA concentration which is sensed by the high-affinity SA receptor NPR4. When bound by SA, it is inactivated and dissociates from NPR1. Consequently, the free NPR1 activates transcription of defense genes, leading to a greater accumulation of SA. This is sensed by the low-affinity SA receptor NPR3. Once it binds SA, it targets NPR1 for proteasomal degradation, therefore suppressing the NPR1-regulated immune response (Fu et al. 2012). When the levels of SA decrease, repression of NPR4 is released and NPR1-activated gene transcription can be swiftly turned off.

The second model proposes NPR1, NPR3 and NPR4 as SA-sensitive transcriptional regulators. (Ding et al. 2018). In the uninduced state, NPR3/NPR4 repress SA-inducible genes at their promoters. With the increase of its concentration, SA binds to NPR3/NPR4 and releases their repressive function. At this stage, NPR1 monomers are already accumulating in the nucleus. In order to activate $P R$ genes transcription, NPR1 must bind SA, a notion previously reported by $\mathrm{Wu}$ and colleagues ( $\mathrm{Wu}$ et al. 2012, Ding et al. 2018). Therefore, changes in SA concentration are sensed by both types of receptors and the transcription of defense genes consequently turned on or off. 


\subsubsection{N-hydroxypipecolic acid is essential for establishment of systemic acquired resistance}

It is clear that SA is indispensable for local and systemic immunity (Wildermuth et al. 2001). However, SA is not transported to the distal tissue, but rather synthesized there upon secondary infection (Vernooij et al. 1994). The transport of a specific mobile signal, an amino acid derivative N-hydroxypipecolic acid (NHP), is essential for the robust activation of SA biosynthesis pathway in the systemic tissue (Mishina and Zeier 2006; Návarová et al. 2012; Chen et al. 2018; Hartmann et al. 2018).

The characterization of the NHP biosynthesis pathway came from a series of studies which aimed to identify mutants that are deficient in SAR (Mishina and Zeier 2006; Návarová et al. 2012; Ding et al. 2016). Like SA biosynthesis, NHP biosynthesis is separated between plastids and the cytosol. It involves three reactions starting from the amino acid L-Lysine. The first step is a transamination catalyzed by ADL1 (AGD2-LIKE DEFENSE RESPONSE PROTEIN1) (Song et al. 2004). This is followed by a reduction by SARD4 (SYSTEMIC ACQUIRED RESISTANCE DEFICIENT4) (Ding et al. 2016, Hartmann et al. 2017). The product of the first two steps, pipecolic acid (Pip), is transported from plastids to the cytosol by EDS5 (Rekhter et al. 2019b). The final step involves the N-hydroxylation of Pip to NHP by FMO1 (FLAVIN-DEPENDENT MONOOXYGENASE1) (Chen et al. 2018; Hartmann et al. 2018).

Interestingly, transcriptional regulation of the NHP biosynthesis genes strongly overlaps with the regulation of SA biosynthesis genes. Transcription factors SARD1 and CBP60g have been demonstrated to bind to the promoters of ALD1, SARD4 and FMO1 upon infection with a pathogen (Sun et al. 2015; Sun et al. 2018). As mentioned before, SARD1 and CBP60g are negatively controlled by CAMTA factors (Kim et al. 2020; Sun et al. 2020) and positively by transcription factor TGA1 (Sun et al. 2018). Furthermore, the latest findings showed that the SA and NHP pathways can mutually amplify each other (Kim et al. 2020; Sun et al. 2020).

The future research will provide more insight into NHP-regulated immunity, especially how the information from NHP is further converted and which are the signaling components downstream of it. 


\subsection{TGA transcription factors}

TGA factors belong to the family of basic leucine-zipper transcription factors characterized by a basic domain, which enables binding to DNA, and a hydrophobic leucine-zipper domain, which enables dimerization of proteins. They were first described as factors binding to the activating sequence1- (as1-) element of Cauliflower Mosaic Virus (CaMV) 35S promoter, which contains two name-giving TGACG motifs (Katagiri et al. 1989). With the release of the Arabidopsis thaliana genome, ten members of the TGA family were assigned (Jakoby et al. 2002). The ten members are grouped according to their sequence similarity into five clades.

The members of clade I have a broad spectrum of activity, while the other clades are associated with either defense responses or plant development (Gatz, 2012). In some of those functions TGA factors are closely linked to their interaction partners from NPR1-like protein family.

\subsubsection{Clade I TGA transcription factors have a broad spectrum of activity}

Clade I TGA factors are represented by two members, TGA1 and TGA4. They were primarily associated with defense responses, but more recently their role has expanded to regulation of nitrate uptake, hyponastic response and plant development.

\subsubsection{TGA1 and TGA4 are redox sensitive transcriptional regulators}

The most important feature of clade I TGA factors are the four redox-sensitive cysteine (Cys) residues. These residues go through redox changes upon treatment with either SA or Snitrosoglutathione (GSNO). The two inner cysteines, Cys260 and Cys266, form a disulfide bridge which is reduced after SA treatment. This brings about the interaction of TGA1 with master regulator of SA signaling, NPR1 (Després et al. 2003). (Figure 1A). Because the reduced form of TGA1 interacts with NPR1 and the interaction stabilizes its binding to as1-element, it was postulated that reduction is a prerequisite for the activation of the protein.

The treatment of TGA1 with GSNO, which serves as donor of nitric oxide (NO), enables interaction with NPR1 in vitro (Lindermayr et al. 2010). Under these conditions, the four cysteines of TGA1, Cys172, Cys260, Cys266 and Cys287, are either S-nitrosylated or S- 


\section{Introduction}

glutathionylated (Figure 1B). The same goes for the cysteines of NPR1 protein (Lindermayr et al. 2010). This interaction of modified proteins enhanced binding affinity of TGA1 to as1element (Després et al. 2003; Lindermayr et al. 2010).
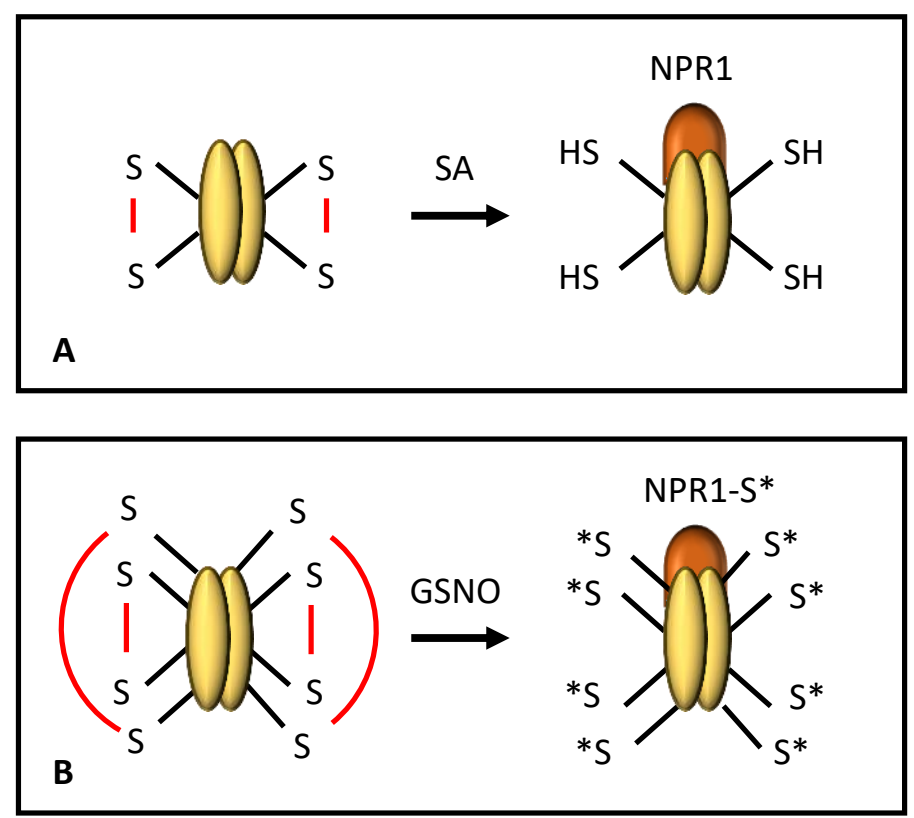

Figure 1 TGA1 is sensitive to redox changes after SA or GSNO treatment.

A In the uninduced state, the two cysteine residues of TGA1 form an intramolecular disulfide bridge. After treatment with SA the disulfide bridge is reduced, and this facilitates interaction with NPR1 (Després et al. 2003)

B In the uninduced state, the two inner and the two outer cysteine residues of TGA1 form an intramolecular disulfide bridge. After treatment with GNSO the four cysteines of TGA1 and cysteines of NPR1 are either S-nitrosylated or S-glutathionylated. The modifications facilitate interaction of the two proteins (Lindermayr et al. 2010).

TGA1 protein is shown in yellow and transcriptional coactivator NPR1 in orange. The black lines represent either reduced (SH) or oxidized (S) sulfhydryl group and the red lines represent disulfide bridge between cysteine residue. The stars represent S-nitrosylated or S-glutathionylated sulfhydryl groups. SA-salicylic acid, GSNO-S-nitrosoglutathione.

However, it was not clear if the redox state of the four critical cysteine residues had any physiological relevance for TGA1 function. To test this, tga1 tga4 mutant was complimented with either wild-type or redox-insensitive version of TGA1 (Li et al. 2019). Surprisingly, both versions of TGA1 protein equally complemented the analyzed phenotype of tga1 tga4. It is important to mention that in the redox-insensitive TGA1 version only the two inner cysteine 
residues were mutated ( $\mathrm{Li}$ et al. 2019). Therefore, the question remains if the additional mutation of the two outer cysteines will have impact on the TGA1 activity.

\subsubsection{TGA1 and TGA4 are important for local and systemic immunity}

Functional characterization of the clade I mutants revealed their importance for induction of $P R$ genes. The single tga1 and the double tga1 tga4 mutant was more susceptibility than wildtype to infection with hemi-biotrophic pathogen Pseudomonas syringae pathovar maculicola ES4326 (Psm) (Kesarwani et al. 2007). Additionally, tga1 tga4 mutant accumulated less SA and Pip than wild-type plant after Psm infection, both locally and systemically (Sun et al. 2018). This was in line with lower induction of genes encoding for regulatory components of SA and Pip biosynthesis, SARD1 and CBP60g. Moreover, TGA1 was shown to bind to SARD1 promoter, which contains multiple TGACG motifs, and directly control the transcription of this gene in Arabidopsis mesophyll protoplast. However, the same was not seen for CBP60g promoter and therefore TGA1 is indirectly responsible for transcription of this gene (Sun et al. 2018).

\subsubsection{TGA1 is proposed to act both upstream and downstream of SA biosynthesis}

TGA1 was initially described as an SA-switchable transcription factor which is activated by reduction of intramolecular disulfide bridge and subsequent interaction with NPR1 (Després et al. 2003). This model puts TGA1 downstream of SA biosynthesis and implies there is a subgroup of NPR1-inducible genes which are TGA1-dependent. In order to find those genes, transcriptome analysis of SA-treated npr1 and tga1 tga4 mutant plants was performed (Shearer et al. 2012). Surprisingly, no such genes were detected. Quite the opposite, a subgroup of differentially regulated genes of npr1 and tga1 tga4 showed reciprocal behavior, meaning that the up-regulated genes in npr1 were down-regulated in tga1 tga4 and vice versa. Therefore, authors questioned the first model and proposed that TGA1 serves as a repressor of NPR1 downstream of SA biosynthesis (Shearer et al. 2012).

However, the latest data described TGA1 and TGA4 as activators of SARD1 and CBP60g, which means they are important upstream of SA biosynthesis and NPR1-dependent signaling (Sun et al. 2018). If they are acting upstream the same pathway, then the expression of NPR1- 


\section{Introduction}

regulated genes should be influenced by the lack of TGA1 and TGA4. As mentioned above, data from Shearer and colleagues does not support this model.

\subsubsection{TGA1 and TGA4 are involved in nitrate uptake, hyponastic response and development}

In addition to their role in the plant immune responses, clade I TGA transcription factors were identified as regulators of nitrate uptake (Alvarez et al. 2014). Transcriptome analysis of hydroponically grown Arabidopsis roots, showed that $97 \%$ of genes differentially expressed in the root of tga1 tga4 mutant were also nitrate-responsive. Moreover, TGA1 was found at the promoters of two nitrate transporters NRT2.1 and NRT2.2 where it presumably activates their transcription (Alvarez et al. 2014).

Furthermore, clade I TGA transcription factors are important for the establishment of hyponastic growth, perceived as the ability of a plant to lift petioles and leaves after treatment with low light (Li et al. 2019). This function was again connected with SA signaling, where SA acts as a negative regulator of hyponastic growth. Because TGA1 can be redox regulated through SA, this phenotype was explored to address the importance of TGA1-redox state. The double tga1 tga4 mutant plants were complemented with either wild-type TGA1 or redoxinsensitive TGA1, with the two inner cysteine residues mutated to mimic the reduced form of the protein (Després et al. 2003; Lindermayr et al. 2010). The two types of complementation lines equally restored wild-type-like hyponastic growth. Additionally, there was no difference in response to SA-application (Li et al. 2019). This data indicates that the redox state of TGA1 is not important for its function in hyponastic growth and SA-repressed hyponastic response.

Recently, interaction of clade I TGA factors with BOP1 and BOP2 was described (Wang et al. 2019). BOP1 and BOP2 fall within the same protein family as NPR1-4 proteins and they play a role in plant development (Hepworth et al. 2005). TGA1/TGA4 and BOP1/BOP2 proteins were shown to have an overlapping expression pattern in organ boundaries in the inflorescence. Moreover, TGA1/TGA4 were found to directly activate transcription of ATH1 (ARABIDOPSIS THALIANA HOMEOBOX GENE1) gene, which is important for BOP1/BOP2-dependent regulation of development (Wang et al. 2019). 


\subsubsection{Clade II and III TGA transcription factors are involved in plant immunity}

Clade II, TGA2, TGA5 and TGA6, and clade III, TGA3 and TGA7, constitutively interact with NPR1, which stabilizes their binding to the as1-element (Zhang et al. 1999; Després et al. 2000; Shearer et al. 2009). Characterization of mutants of clade II and clade III revealed their involvement in basal (TGA3) and systemic (TGA2/TGA5/TGA6) immunity (Kesarwani et al. 2007). TGA3 loss-of-function mutant was more susceptible than wild-type to local infection with Psm, while the triple tga2 tga5 tga6 mutant had npr1-like defects in SAR (Zhang et al. 2003; Kesarwani et al. 2007). The immune deficiency was in line with the mutant's inability to induce $P R 1$ gene to the wild-type levels after SA treatment. The basal levels of $P R 1$ were lower in tga 3 than in the wild-type plant which went in hand with the susceptibility phenotype (Kesarwani et al. 2007). However, tga2 tga5 tga6 mutant had higher basal levels of PR1 than the wild-type plant (Kesarwani et al. 2007; Zhang et al. 2005). This implied that clade II TGA factors can also be negative regulators of $P R 1$ gene expression, a function which was later assigned to TGA2 protein. TGA2 is constitutively recruited to the $P R 1$ promoter where it binds both positive and negative region of as1-element (Rochon et al. 2006). The latest model proposes that TGA2 recruits either NPR1 or NPR3/NPR4 to the NPR1-induced promoter regions, dependent on the SA concentration (Ding et al. 2018). At the promoter, NPR3/NPR4 repress and NPR1 induces expression of $P R$ genes, respectively.

In addition to their role in SA-regulated defense signaling, clade II TGA transcription factors are involved in hormonal crosstalk between SA and JA/ET pathways (Zander et al. 2010; Zander et al. 2014) and clade III TGA transcription factors in cytokinin-mediated SAR (Choi et al. 2010).

\subsubsection{Clade IV and V TGA transcription factors are involved in plant development}

Members of clade IV and $\mathrm{V}$ are important for development of reproductive organs of Arabidopsis thaliana. The two members of clade IV, TGA9 and TGA10, are redundantly required for anther development, and the absence of the factors results in male sterility (Murmu et al. 2010). The sole member of clade V, PAN, is a negative regulator of petal development (Chuang et al. 1999). The loss of PAN leads to changes in the regular Arabidopsis flower pattern from tetramerous to pentamerous. The repression of petal development is established through interaction between PAN and BOP1/BOP2 (Hepworth et al. 2005). 


\subsection{Glutaredoxins}

Glutaredoxins (GRXs) are small ubiquitous proteins functioning as either oxidoreductases or iron sulfur complex binding proteins (Ströher and Millar 2012; Gutsche et al. 2015). They form the thioredoxin protein superfamily together with thioredoxins, protein-disulfide-isomerases, glutathione peroxidases and glutathione transferases (Martin 1995). All members of the superfamily contain conserved structural thioredoxin fold, which is characterized by four stranded $\beta$-sheets surrounded by three $\alpha$-helices. Additional features characteristic to GRXs are (i) a CXXC or CXXS active site motif (where $\mathrm{X}$ is any and $\mathrm{S}$ is serine amino acid residue) and (ii) a specific binding site for a glutathione (GSH) tripeptide (Gutsche et al. 2015).

In general, these proteins maintain the free cysteine residues of intracellular proteins in a reduced state. Under unfavorable conditions such as oxidative stress, the thiol group of a cysteine residue is oxidized by the reactive oxygen or nitrogen species (ROS or NOS). Because the thiol group is involved in intra- and intermolecular disulfide bridge formation, sulfenylation, nitrosylation and further oxidations, changes of this group can alter the activity of a protein. Therefore, cysteine residues are protected through formation of mixed disulfides with GSH in the process termed glutathionylation (Ströher and Millar 2012). Once the oxidative stress has passed, cysteine residues are reduced through deglutathionylation by GRXs.

\subsubsection{Glutaredoxins in model plant Arabidopsis thaliana}

GRXs are usually divided into three classes according to the active site motif which is crucial for their redox and FeS cluster binding functions. Class I (CPYC-type) and class II (CGFS-type) GRXs are found in almost all pro-and eukaryotes, while class III (CC-type) is restricted to land plants (Meyer et al. 2008; Couturier et al. 2009; Ströher and Millar 2012).

\subsubsection{Class I and class II glutaredoxins}

When describing the function of GRXs, it is usually the function of class I and class II glutaredoxins that is described. As stated above, their main function is deglutathionylation of mixed disulfides and formation of iron-sulfur clusters. Although majority of the described class I and II GRXs belong to organisms other than plants, their function seems to be rather conserved (Ströher and Millar 2012). 


\section{Introduction}

The main differences between class I and II GRXs are mechanisms of deglutathionylation reaction. Class I consists of GRXs which use both mono- and dithiol mechanism while class II exclusively contains monothiol GRXs (Ströher and Millar 2012). The monothiol mechanism requires only $\mathrm{N}$-terminal Cys of the active site to reduce the mixed disulfide. On the other hand, the dithiol mechanism, which involves a formation of intramolecular disulfide bridge in the active site, requires both $\mathrm{N}$ - and $\mathrm{C}$-terminal Cys residues of the active site.

\subsubsection{Class III CC-type glutaredoxins interact with TGA transcription factors}

The family of CC-type glutaredoxins, also known as ROXYs, consists of twenty-one members in model plant Arabidopsis thaliana. The gene family expanded since the appearance of the first land plants, indicating that these proteins might execute land plant-specific functions. Following the nomenclature from Li and colleagues, members of CC-type GRXs were named according to the sequence similarity to the pioneer ROXY1 (Figure 2) (Xing et al. 2005; Li et al. 2009).

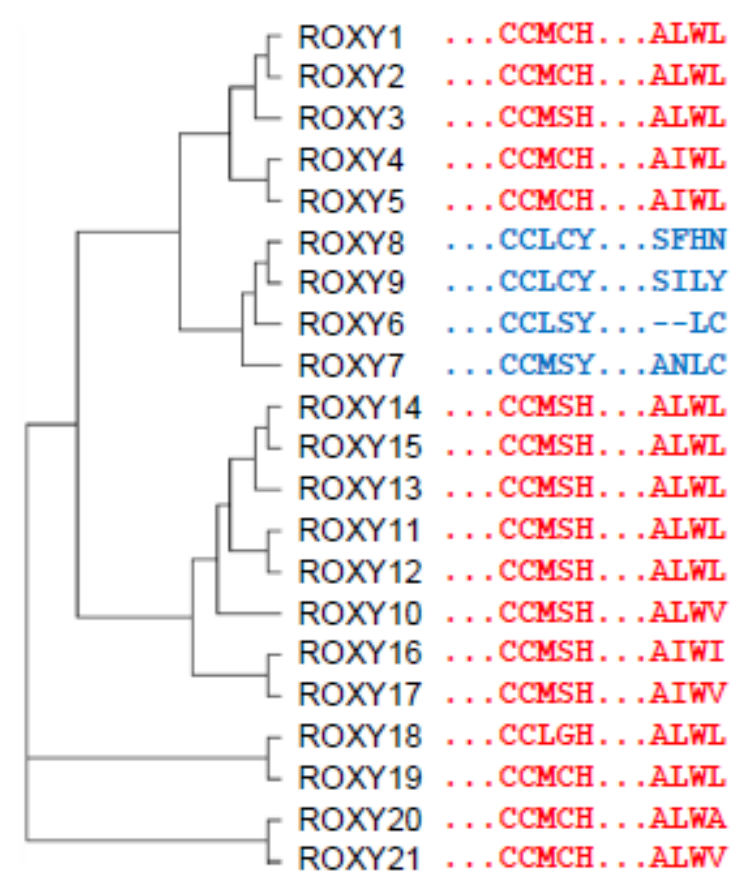

Figure 2 Alignment of the active sites and the C-terminal four amino acids of ROXYs.

ROXYs $6,7,8$, and 9 (shown in blue) are distinguished from all other ROXYs by the $Y$ following the active site and by the lack of ALWL-related sequences at C-terminal site (Taken from Li et al, 2018). 
As shown in the Figure 2, the ROXYs differ in respect to their active site and C-terminal motif. Most of the information about ROXYs was gathered from genetic studies using either loss-offunction mutants or ectopically expressed proteins. From this data it was noticed that ROXYs functions are tied to their interaction partners, TGA transcription factors. Therefore, this clade of GRXs will be described in more detail.

\subsubsection{ROXY1 and ROXY2 interact with class IV and V TGA transcription factor to regulate flower and anther development}

The first information about CC-type GRXs came from the study of the ROXY1 gene (Xing et al. 2005). The roxy1 mutant was defective in flower development and this was explored to map functional groups of the protein. It was shown that the $\mathrm{N}$-terminal cysteine residue of the active site (CCMC) and the glycine residue of the putative GSH-binding site is important for ROXY1 protein activity ( Xing et al. 2005; Xing and Zachgo 2008). Since the protein is localized in the nucleus and this is essential for its function, the screen for potential nuclear localized interaction partners was performed. Four members of TGA protein family, namely TGA2, TGA3, TGA7 and PAN, were identified in the yeast two-hybrid analysis (Li et al. 2009). Out of the four, only PAN had an overlapping expression domain as ROXY1. As mentioned before, PAN was described as a negative regulator of flower development (Chuang et al. 1999). Further analysis showed that ROXY1 serves as a repressor of PAN transcription factor, and its function enables development of a typical tetramerous flower pattern in Arabidopsis thaliana (Chuang et al. 1999; Li et al. 2009).

In addition to its role in flower development, ROXY1 is, redundantly with ROXY2, required for proper anther development (Xing et al. 2005). This defect in anther development is also observed in tga9 tga10 double mutant. Although the interaction between ROXY1/ ROXY2 and TGA9/TGA10 transcription factors was described, the mechanism which leads to the anther defects remains unclear (Murmu et al. 2010). However, both double mutants shown the same phenotype, therefore it is plausible to think that ROXY1/ ROXY2 act as positive regulators of TGA9/TGA10. 


\subsubsection{ROXY-type glutaredoxins interact with class II TGA transcription factors}

The CC-type glutaredoxin ROXY19 contains ALWL motif on its C-terminal site and it interacts with TGA2, TGA5 and TGA6 (Ndamukong et al. 2007). As mentioned above, clade II TGA factors are involved in hormonal cross-talk between SA and JA/Et pathway. When ectopically expressed, ROXY19 mimics the TGA2/TGA5/TGA6-dependent cross-talk between the SA and the JA/Et pathway (Ndamukong et al. 2007). Because ROXY19 gene is induced by SA, it was hypothesized that this protein is responsible for TGA2-dependent JA/Et pathway suppression. This phenomenon can be observed as repression of JA/Et-responsive ORA59 promoter and was therefore utilized to test redundancy of the CC-type GRXs. Although TGA2 interacted with all seventeen tested ROXYs, only ROXYs which contained ALWL motif on the C-terminus were able to suppress ORA59 (Zander et al. 2012). As expected, mutation of ALWL to ALWA in ROXY19 led to a loss-of-function phenotype and ALWA to ALWL in ROXY20 led to gain-offunction phenotype in plant protoplasts.

The ALWL motif was later shown to be important for interaction of ROXYs with a well-known transcriptional co-repressor TPL (TOPLESS) (Uhrig et al. 2017). TPL and TPR (TPL-RELATED PROTEINS) are characterized by conserved sequences which are responsible for interaction with EAR domain of transcription factors or adaptor proteins (Pauwels et al. 2010). Although ROXYs lack the EAR domain, it was shown in yeast-two-hybrid that they interact with TPL through ALWL motif. Therefore, it was proposed that ROXY19-mediated transcriptional repression is established through ternary complex consisting of TGA2-ROXY19-TPL (Uhrig et al. 2017). However, it remains unclear why the repression is also dependent on the active site, which is dispensable for both TGA and TPL binding.

\subsubsection{ROXY type glutaredoxins interact with class I TGA transcription factors to repress their functions}

ROXY9 does not contain an ALWL motif and it interacts with clade I TGA transcription factors (Li et al. 2019). Ectopically expressed ROXY9 mimics tga1 tga4 double mutant's susceptibility to biotrophic pathogen and its defects in hyponastic growth (M. Muthreich PhD thesis. 2014; Li et al. 2019). The latter phenotype was used to address the importance of the active site cysteine residues for the ROXY9 protein function. Because ROXY9 has three cysteine residues 


\section{Introduction}

in the active site, three different active site mutants were prepared. The hyponastic growth phenotype was lost when the first (CCLC to SCLC) or the second (CCLC to CSLC) active site cysteine was mutated to serine residue. The mutant where the third cysteine residue was mutated (CCLC to CCLS) behaved as an ectopically expressed wild-type ROXY9. Nevertheless, the interaction of either mutant with TGA1 was not disturbed (Li et al. 2019). Therefore, it was shown that the first and the second Cys residue of the active site motif is important for the ROXY9-mediated repression of TGA1/TGA4. However, the underlying mechanism of repression is not yet known (Uhrig et al. 2017; Li et al. 2019).

In order to better understand the function of ROXY9, a ROXY9 loss-of-function mutant was generated. The mutation was expected to release ROXY9-mediated repression of TGA1/TGA4. However, this was not observed, and the mutant had a wild-type-like behavior. As an explanation, it was proposed that the other ROXYs that lack ALWL motif, namely ROXY6, ROXY7 and ROXY8, act redundantly to ROXY9. 


\subsection{Aim of the thesis}

Since TGA1 is modulated by SA, what is its role downstream of SA?

TGA1/TGA4 are established regulators of SA-biosynthesis (Sun et al. 2018). Interestingly, SA accumulation changes the redox state of TGA1. Upon SA-treatment, disulfide bridge formed between the two TGA1-cysteine residues can be reduced, facilitating TGA1-NPR1 interaction (Després et al. 2003). Moreover, Cys172 and Cys287 of TGA1 can be gluthationylated and nitroslytated in vitro (Lindermayr et al. 2010). It was postulated that TGA1 reduction might lead to the activation of the protein. Nevertheless, function of TGA1/TGA4 downstream of SAsignaling has not been described so far. One of the aims of this project was to elucidate TGA1/TGA4-dependent signaling downstream of SA.

\section{Are the critical cysteine residues important for the role of TGA1 downstream of SA?}

Because TGA1 protein is redox regulated after SA treatment, we sought to investigate the importance of TGA1-redox state to complement the expression of TGA1/TGA4-dependent genes downstream of SA. To do so, we complemented tga1 tga4 mutant with either genomic clone of TGA1 or a mutant which mimics the reduced-active form of the protein. This complementation was performed by Katrin Treffon.

\section{Do highly expressed glutaredoxins in tga1 tga4 mutant suppress the activity of TGA2/TGA5/TGA6?}

CC-type glutaredoxin ROXY19 have been shown to negatively regulated class II TGA transcription factors (Ndamukong et al. 2007; Zander et al. 2014; Huang et al. 2016). The repressive function of ROXY19 has been connected to its C-terminal ALWL motif (Zander et al. 2012). The ALWL motif is important for the interaction with the transcriptional co-repressor TOPLESS, which fits to the findings that ROXYs are negative regulators of TGA function (Uhrig et al. 2017). Microarray data from tga1 tga4 mutant revealed elevated expression of ROXYs which contains ALWL motif (M. Muthreich PhD thesis, 2014). We hypothesized that they could 


\section{Introduction}

act as repressors of TGA2/TGA5/TGA6 in tga1 tga4 mutant background. To test this hypothesis, we used CRISPR-Cas9 technology to delete a gene cluster containing five ROXY genes in tga1 tga4 mutant background.

Since TGA1 is repressed by ectopically expressed ROXY9, does ROXY9 loss-of-function have a reverse effect?

ROXY9 belongs to those four ROXYs (ROXY6, ROXY7, ROXY8 and ROXY9) that do not contain an ALWL motif. When over-expressed, ROXY9 represses TGA1/TGA4-regulated defense responses (M. Muthreich PhD thesis, 2014). One of the aims of the project was to construct a roxy6 roxy7 roxy8 roxy9 quadruple mutant to test if the loss-of-function releases the repression of TGA1/TGA4 activity. 


\section{Materials and Methods}

\subsection{Materials}

\subsubsection{Organisms}

\subsubsection{Bacteria}

\begin{tabular}{|c|c|c|}
\hline BACTERIAL STRAIN & DESCRIPTION & REFERENCE \\
\hline Escherichia coli DH5 $\alpha$ & $\begin{array}{l}\text { F- } \$ 80 \text { lacZ } \Delta \mathrm{M} 15 \Delta(\text { lacZYA-argF) } \\
\text { U169 recA1 endA1 hsdR17(rk-, mk+) } \\
\text { phoA supE44 thi-1 gyrA96 relA1 } \lambda-\end{array}$ & Thermo Fisher Scientific \\
\hline Agrobacterium tumefaciens GV3101 & C58; RifR; GentR & (Koncz and Schell 1986) \\
\hline $\begin{array}{l}\text { Pseudomonas syringae pv. } \\
\text { maculicola ES4326 }\end{array}$ & RifR & (Whalen et al. 1991) \\
\hline $\begin{array}{l}\text { Pseudomonas syringae } p v . \\
\text { Tomato DS3000 } \triangle a v r P t o / P t o B\end{array}$ & 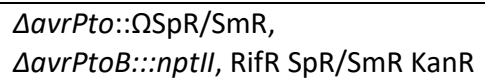 & (Lin and Martin 2005) \\
\hline
\end{tabular}

\subsubsection{Plants}

\begin{tabular}{|c|c|c|}
\hline GENOTYPE & DESCRIPTION & REFERENCE \\
\hline Col-0 & Arabidopsis thaliana Columbia-0 (Col-0) & TAIR \\
\hline $\operatorname{tga1} \operatorname{tga} 4$ & $\begin{array}{l}\text { tga1 and tga4 double mutant in Col-0 } \\
\text { background }\end{array}$ & (Kesarwani et al. 2007) \\
\hline $\operatorname{tga} 2 \operatorname{tga} 5$ tga 6 & $\begin{array}{l}\text { tga2, tga } 5 \text { and tga } 6 \text { triple mutant in Col-0 } \\
\text { background }\end{array}$ & (Zhang et al. 2003) \\
\hline npr1 & npr1 single mutant in Col-0 background & (Cao et al. 1994) \\
\hline sard1 cbp60g & $\begin{array}{l}\text { sard1 and } c b p 60 g \text { double mutant in Col-0 } \\
\text { background }\end{array}$ & (Wang et al. 2011) \\
\hline $\operatorname{sid} 2-2$ & $\begin{array}{l}\text { isc1 gene single mutant in Col-0 } \\
\text { background }\end{array}$ & (Wildermuth et al. 2001) \\
\hline sid2 tga1 tga4 & $\begin{array}{l}\text { isc1, tga1 and tga } 4 \text { triple mutant in Col-0 } \\
\text { background }\end{array}$ & M. Muthreich PhD thesis (2014) \\
\hline sid2 tga 2 tga 5 tga 6 & $\begin{array}{l}\text { ics } 1 \text { and tga } 2 \text { tga } 5 \text { tga } 6 \text { quadruple mutant } \\
\text { obtained by cross }\end{array}$ & K. Rindermann PhD thesis (2010) \\
\hline sid2 npr1 & $\begin{array}{l}\text { ics1 and npr1 double mutant obtained by } \\
\text { cross }\end{array}$ & Gatz group, 2018 \\
\hline roxy6 & roxy 6 single mutant in Col-0 background & $\begin{array}{l}\text { This work, with help of Dr. } \\
\text { Corinna Thurow }\end{array}$ \\
\hline roxy7 & roxy7 single mutant in Col-0 background & $\begin{array}{l}\text { This work, with help of Dr. } \\
\text { Corinna Thurow }\end{array}$ \\
\hline roxy9 mutant & roxy9 mutant in Col-0 background & F. Jung Master Thesis (2016) \\
\hline roxy6 roxy7 & $\begin{array}{l}\text { roxy6 and roxy7 double mutant in Col-0 } \\
\text { background }\end{array}$ & This work \\
\hline roxy6 roxy7 roxy9 & $\begin{array}{l}\text { roxy6, roxy7 and roxy9 triple mutant in Col- } \\
0 \text { background obtained by cross }\end{array}$ & This work \\
\hline
\end{tabular}




\begin{tabular}{|c|c|c|}
\hline roxy6 roxy7 roxy8 roxy9 & $\begin{array}{l}\text { roxy6, roxy7, roxy8 and roxy9 quadruple } \\
\text { mutant in Col-0 background obtained by } \\
\text { cross }\end{array}$ & Gatz group, 2019 \\
\hline $\operatorname{roxy} 11-15$ & $\begin{array}{l}\operatorname{roxy} 11, \operatorname{roxy} 12, \operatorname{roxy} 13, \operatorname{roxy} 14 \text { and roxy15 } \\
\text { pentuple mutant in Col-0 background }\end{array}$ & $\begin{array}{l}\text { This work, with help of Dr. } \\
\text { Corinna Thurow and Anna } \\
\text { Hermann }\end{array}$ \\
\hline roxy11-15 tga1 tga4 & $\begin{array}{l}\operatorname{roxy} 11, \operatorname{roxy} 12, \operatorname{roxy} 13, \operatorname{roxy} 14, \operatorname{roxy} 15, \\
\operatorname{tga} 1 \text { and tga4 heptuple mutant in Col-0 } \\
\text { background }\end{array}$ & $\begin{array}{l}\text { This work, with help of Dr. } \\
\text { Corinna Thurow and Anna } \\
\text { Hermann }\end{array}$ \\
\hline Col-0:: TGA1prom:GUS & $\begin{array}{l}\text { Expressing the GUS gene under the control } \\
\text { of } T G A 1 \text { promoter in } \mathrm{Col}-\mathrm{O}\end{array}$ & (Wang et al. 2019) \\
\hline tga1 tga4:: TGA1prom:HA-3'UTR & $\begin{array}{l}\text { Expressing the } 3^{\prime} U T R \text { of } T G A 1 \text { gene under } \\
\text { the control of the native promoter, } \mathrm{N} \text { - } \\
\text { terminal } 1 \text { x HA-tag }\end{array}$ & K. Treffon PhD thesis (2018) \\
\hline tga1 tga4:: TGA1prom:HA-TGA1g & $\begin{array}{l}\text { Expressing the TGA1 gene under the } \\
\text { control of the native promoter, } \mathrm{N} \text {-terminal } \\
1 \times \mathrm{HA} \text {-tag }\end{array}$ & K. Treffon PhD thesis (2018) \\
\hline tga1 tga4:: TGA1prom:HA-TGA1gr & $\begin{array}{l}\text { Expressing the TGA1 gene with } 4 \text { cysteine } \\
\text { residues mutated (172C-172N, } 260 \mathrm{C}-260 \mathrm{~N} \text {, } \\
266 \mathrm{C}-266 \mathrm{~S}, 287 \mathrm{C}-287 \mathrm{~S}) \text { under the control } \\
\text { of the native promoter, N-terminal } 1 \times \mathrm{HA}- \\
\text { tag }\end{array}$ & K. Treffon PhD thesis (2018) \\
\hline
\end{tabular}

\subsubsection{Oligonucleotides and plasmids}

\subsubsection{Oligonucleotides for CRISPR-Cas9 cloning}

\begin{tabular}{l|l} 
PRIMER & SEQUENCE (5' -3') \\
\hline CRISPR-ROXY11-15 A fwd & ATATATGGTCTCTGATTGAAAGATGATCTCCGAGAAGTGTTTTAGAGCTAGAAATAGCAAG \\
\hline CRISPR-ROXY11-15 B fwd & ATATATGGTCTCTGATTGAAGACTCTCTTCTTAGACCTGTTTTAGAGCTAGAAATAGCAAG \\
\hline CRISPR-ROXY11-15 C fwd & ATATATGGTCTCTGATTGATGGAGACTCATGACTTGATGTTTTAGAGCTAGAAATAGCAAG \\
\hline CRISPR-ROXY11-15 A rev & AATAATGGTCTCTAAACACTTCTCGGAGATCATCTTTCAATCTCTTAGTCGACTCTACC \\
\hline CRISPR-ROXY11-15 B rev & ATTATTGGTCTCTAAACAGGTCTAAGAAGAGAGTCTTCAATCTCTTAGTCGACTCTACC \\
\hline CRISPR-ROXY11-15 C rev & ATTATTGGTCTCTAAACATCAAGTCATGAGTCTCAATCAATCTCTTAGTCGACTCTACC \\
\hline sgRNA and 26ter rev & CGATCTGGAAAATTTTGCAAAAAAAAGCACCGACTCG \\
\hline sgRNA and 26ter fwd & CGAGTCGGTGCTTTTTTTTGCAAAATTTTCCAGATCG \\
\hline 26ter and 2 pro rev & CAGTAGTTTGGATTAATATTGGTTTATCTCATCGGAACTGC \\
\hline 26ter and 29pro fwd & CCGATGAGATAAACCAATATTAATCCAAACTACTGCAGCCTGAC \\
\hline CRISPR-ROXY6 A fwd & GAGAGAGAAGACATGATTGAATGTCGTCCGAAAAAGGGGGTTTTAGAGCTAGAAATAGCA \\
\hline CRISPR-ROXY6 B fwd & GAGAGAGAAGACATGATTGGACAAACAACAGGAGCTCTGTTTTAGAGCTAGAAATAGCAA \\
\hline CRISPR-ROXY7 A fwd & GAGAGAGAAGACATGATTGCTCGTGTTGCATGTCCTATGGTTTTAGAGCTAGAAATAGCA \\
\hline CRISPR- ROXY7 B fwd & GAGAGAGAAGACATGATTGAAAAGTACTTGGACCGCATGTTTTAGAGCTAGAAATAGCAA \\
\hline CRISPR-ROXY6 A rev & GAGAGAGAAGACATAACCCCCCTTTTTCGGACGACATTCAATCTCTTAGTCGACTCTACC \\
\hline CRISPR- ROXY6 B rev & GAGAGAGAAGACATAACAGAGCTCCTGTTGTTTGTCCAATCTCTTAGTCGACTCTACC \\
\hline CRISPR-ROXY7 A rev & GAGAGAGAAGACATAACCATAGGACATGCAACACGAGCAATCTCTTAGTCGACTCTACC \\
\hline CRISPR- ROXY7 B rev & GAGAGAGAAGACATAACATGCGGTCCAAGTACTTTTCAATCTCTTAGTCGACTCTACC
\end{tabular}




\subsubsection{Oligonucleotides for CRISPR-Cas9 genotyping}

\begin{tabular}{l|l|l} 
PRIMER PAIR & FORWARD SEQUENCE $\left(\mathbf{5}^{\prime} \mathbf{-} \mathbf{3}^{\prime} \mathbf{)}\right.$ & REVERSE SEQUENCE $\left(\mathbf{5}^{\prime} \mathbf{-} \mathbf{3}^{\prime} \mathbf{)}\right.$ \\
\hline ROXY15 & CATCCAACGCATAATGTCATAGC & CATCCTTGATTGGTTTCATGACAT \\
\hline ROXY14 & CATCGAACCCATAATATCATATCCTTACG & CGAAATCAGTACCCTGCCTCATAATC \\
\hline ROXY13 & GACTTCAAGTTCTCTAGCTTACCAATTTCAC & ACAGATTAAAATGGGAAATGGAAATCC \\
\hline ROXY12 & ATCCTCCGTGAATCACTTTCTTCAG & AATAACGTCGACGCATGTGATCTTAG \\
\hline ROXY11 & CTAATCTAGCATTTTGACCAAACACACC & TGCACGTGTATTCATTTCTAGATGC \\
\hline ROXY6 & TTTCTTGTTGCATAGTTTGGGTCAC & TAAATATGGCTTCACTAGGGGAACG \\
\hline ROXY7 & ACCCTCTTTTCTTCAAACAGGAACC & AGACAAGAAGACAAATCGTTGCCTG \\
\hline BASTA & GGTCTGCACCATCGTCAACCAC & CAGCTGCCAGAAACCCACGTC
\end{tabular}

\subsubsection{Oligonucleotides for real time PCR}

\begin{tabular}{l|l|l} 
PRIMER PAIR & FORWARD SEQUENCE $\left(\mathbf{5}^{\prime} \mathbf{-} \mathbf{3}^{\prime}\right)$ & REVERSE SEQUENCE $\left(\mathbf{5}^{\prime} \mathbf{-} \mathbf{3}^{\prime} \mathbf{)}\right.$ \\
\hline DLO1 & AATATCGGCGACCAAATGC & CGCTCGTTCTCGGTGTTTAC \\
\hline UBQ5 & GACGCTTCATCTCGTCC & GTAAACGTAGGTGAGTCCA \\
\hline PR1 & CTGACTTTCTCCAAACAACTTG & CAAACTAAACAATAAACCATACCATAA \\
\hline SARD1 & TCAAGGCGTTGTGGTTTGTG & CGTCAACGACGGTATGTTTC
\end{tabular}

\subsubsection{Oligonucleotides for real time PCR from QuantiTect}

\begin{tabular}{l|l} 
PRIMER PAIR -QuantiTect & CATALOGUE NUMBER \\
\hline BGL2 & QT00793730
\end{tabular}

\subsubsection{Oligonucleotides for GATEWAYTM cloning}

\begin{tabular}{|c|c|c|}
\hline PRIMER PAIR & FORWARD SEQUENCE (5'-3') & REVERSE SEQUENCE $\left(5^{\prime}-3^{\prime}\right)$ \\
\hline DLO1 promoter & $\begin{array}{l}\text { GGGGACAAGTTTGTACAAAAAAGCAGGCTAACTA } \\
\text { ATTTACGTGTTCTCCACCA }\end{array}$ & $\begin{array}{l}\text { GGGGACCACTTTGTACAAGAAAGCTGGGTGCCAT } \\
\text { TTAATGTGTTTGGTAATGTAATTTTG }\end{array}$ \\
\hline TGA1noSTOP & $\begin{array}{l}\text { GGGGACAAGTTTGTACAAAAAAGCAGGCTCAACA } \\
\text { ATGAATTCGACATCGACACATTTT }\end{array}$ & $\begin{array}{l}\text { GGGGACCACTTTGTACAAGAAAGCTGGGTCCGTT } \\
\text { GGTTCACGATGTCGA }\end{array}$ \\
\hline TGA2n & $\begin{array}{l}\text { GGGGACAAGTTTGTACAAAAAAGCAGGCTCAACA } \\
\text { ATGGCTGATACCAGTCCGAG }\end{array}$ & $\begin{array}{l}\text { GGGGACCACTTTGTACAAGAAAGCTGGGTCCTCTC } \\
\text { TGGGTCGAGCAAGC }\end{array}$ \\
\hline
\end{tabular}

\subsubsection{Plasmids for CRISPR-Cas9}

\begin{tabular}{l|l} 
PLASMID & SOURCE \\
\hline pB-CRISPR-AT2S3pGFP & Dr Corinna Thurow \\
\hline PB-CRISPR-ROXY11-15AC8 & This work, with help of Dr Corinna Thurow \\
\hline PB-CRISPR-ROXY11-15CA3-1 & This work, with help of Dr Corinna Thurow \\
\hline PB-CRISPR-ROXY11-15AB2 & This work, with help of Dr Corinna Thurow \\
\hline PB-CRISPR-ROXY11-15BA1 & This work, with help of Dr Corinna Thurow \\
\hline PB-CRISPR-ROXY11-15BC2 & This work, with help of Dr Corinna Thurow \\
\hline
\end{tabular}




\section{Materials and Methods}

\begin{tabular}{l|l}
\hline PB-CRISPR-ROXY11-15CB2 & This work, with help of Dr Corinna Thurow \\
\hline PB-CRISPR-ROXY6A7A -2 & This work, with help of Dr Corinna Thurow \\
\hline PB-CRISPR-ROXY6A7A -1 & This work, with help of Dr Corinna Thurow \\
\hline PB-CRISPR-ROXY6A7B -1 & This work, with help of Dr Corinna Thurow \\
\hline PB-CRISPR-ROXY6A7B -2 & This work, with help of Dr Corinna Thurow \\
\hline PB-CRISPR-ROXY6B7A -1 & This work, with help of Dr Corinna Thurow \\
\hline PB-CRISPR-ROXY6B7A -1 & This work, with help of Dr Corinna Thurow \\
\hline PB-CRISPR-ROXY6B7B -2 & This work, with help of Dr Corinna Thurow \\
\hline PB-CRISPR-ROXY7A6A -1 & This work, with help of Dr Corinna Thurow
\end{tabular}

\subsubsection{Plasmids for GATEWAYTM cloning}

\begin{tabular}{l|l} 
PLASMID & SOURCE \\
\hline pDONR207 & Gatz group, Dr. Corinna Thurow and Anna Hermann \\
\hline pDONR207-DLO1pro & This work \\
\hline pDONR207-TGA1nOSTOP & This work \\
\hline pDONR207-TGA2nOSTOP & This work
\end{tabular}

\subsubsection{Plasmids for dual-luciferase reporter assay}

\begin{tabular}{l|l} 
PLASMID & SOURCE \\
\hline pBGWL7-DLO1 & This work \\
\hline pBGWL7-DLO1-T-mut & Gatz group, Dr. Corinna Thurow and Anna Hermann \\
\hline pBGWL7-DLO1-A-mut & Gatz group, Dr. Corinna Thurow and Anna Hermann \\
\hline pUBQ10-TGA1-3HA-Strep & This work \\
\hline pUBQ10-TGA1-VP & Gatz group, Dr. Corinna Thurow and Anna Hermann \\
\hline pUBQ10-TGA2-3HA-Strep & This work \\
\hline pUBQ10-TGA2-VP & Gatz group, Dr. Corinna Thurow and Anna Hermann \\
\hline pUBQ10-SARD1-3HA-Strep & Gatz group \\
\hline pUBQ10-SARD1-VP & Gatz group, Dr. Corinna Thurow and Anna Hermann \\
\hline pUBQ10-3HA-Strep & Gatz group \\
\hline renillaLUC & Gatz group
\end{tabular}

\subsubsection{Chemicals, antibodies and kits}

\subsubsection{Chemicals}

\begin{tabular}{l|l} 
CHEMICAL & SOURCE \\
\hline 2-[N-Morpholino]-ethanesulfonic acid (MES) & Carl Roth GmbH \& Co. KG \\
\hline $\begin{array}{l}\text { 2-Amino-2-hydroxymethyl-propane-1,3-diol } \\
\text { (TRIS) }\end{array}$ & Sigma \\
\hline 2-Mercaptoethanol & Carl Roth GmbH \& Co. KG \\
\hline
\end{tabular}




\section{Materials and Methods}

\begin{tabular}{|c|c|}
\hline Acrylamide/Bisacrylamide & Sigma-Aldrich \\
\hline Agarose & Biozym \\
\hline Ammonium persulfate (APS) & Biometra \\
\hline Ammonium thiocyanate & Sigma-Aldrich \\
\hline BASTA & Bayer \\
\hline Bovine serum albumin (BSA) & Serva \\
\hline Bromophenol blue & Carl Roth GmbH \& Co. KG \\
\hline Chloroform & Carl Roth GmbH \& Co. KG \\
\hline Citric acid & Carl Roth GmbH \& Co. KG \\
\hline di-Sodium hydrogen phosphate & Carl Roth GmbH \& Co. KG \\
\hline Dithiotheritol (DTT) & Carl Roth GmbH \& Co. KG \\
\hline Ethanol & W. Krannich GmbH \& Co. KG \\
\hline Ethidiumbromide & Carl Roth GmbH \& Co. KG \\
\hline Ethylenediaminetetraacetic acid (EDTA) & Applichem \\
\hline Fat-free milk powder & commercial \\
\hline Fluoresceine & BioRad \\
\hline Formaldehyde, $37 \%$ & Sigma \\
\hline Glycerin & Carl Roth GmbH \& Co. KG \\
\hline Glycerol & Sigma \\
\hline Glycine & Carl Roth GmbH \& Co. KG \\
\hline Glycogen & Sigma-Aldrich Chemie GmbH \\
\hline Guanidinium thiocyanate & Sigma \\
\hline Hydrochloric acid & Carl Roth GmbH \& Co. KG \\
\hline Lithium chloride & Carl Roth GmbH \& Co. KG \\
\hline Magnesium chloride & Hilmer Brauer \\
\hline Murashige and Skoog medium (MS medium) & Duchefa \\
\hline N,N-Dimethylformamide (DMF) & Sigma \\
\hline NP-40 & Fluka \\
\hline Orange G & Sigma \\
\hline Peptone BD & Biosciences \\
\hline Phenol & Sigma \\
\hline Phenyl methyl sulfonyl fluoride (PMSF) & Sigma-Aldrich Chemie GmbH \\
\hline Potassium chloride & Carl Roth GmbH \& Co. KG \\
\hline Potassium chloride & Carl Roth GmbH \& Co. KG \\
\hline Potassiumferricyanide $\left(\mathrm{K}_{3} \mathrm{Fe}(\mathrm{CN})_{6}\right)$ & Sigma \\
\hline Potassiumferrocyanide $\left(\mathrm{K}_{4} \mathrm{Fe}(\mathrm{CN})_{6}\right)$ & Sigma \\
\hline Protease inhibitors & Sigma \\
\hline Select Agar & Life Technologies \\
\hline Select yeast extract & Gibco BRL \\
\hline Silwet L-77 & (Momentive) Kurt Obermeier Gmbh \& Co. KG \\
\hline Sodium acetate & Carl Roth GmbH \& Co. KG \\
\hline Sodium chloride & Carl Roth GmbH \& Co. KG \\
\hline
\end{tabular}




\begin{tabular}{l|l}
\hline $\begin{array}{l}\text { Sodium dehydrogen phosphate } \\
\text { monohydrate }\end{array}$ & Carl Roth GmbH \& Co. KG \\
\hline Sodium deoxycholate & Sigma \\
\hline Sodium dodecyl sulfate (SDS) & Carl Roth GmbH \& Co. KG \\
\hline Sodium hypochlorite & Carl Roth GmbH \& Co. KG \\
\hline Sodium salicylate & Sigma \\
\hline Sucrose & Duchefa \\
\hline Tetra methyl ethylene diamine (TEMED) & Carl Roth GmbH \& Co. KG \\
\hline $\begin{array}{l}\text { TRIS saturated Phenol : Chloroform : Isoamyl } \\
\text { Alcohol (25:24:1) (v/v/v) }\end{array}$ & Carl Roth GmbH \& Co. KG \\
\hline Tri-sodium-citrate & Carl Roth GmbH \& Co. KG \\
\hline Triton X-100 & Carl Roth GmbH \& Co. KG \\
\hline Urea & Sigma \\
\hline X-Gluc & BioTech Trade \& Service GmbH
\end{tabular}

\subsubsection{Growth media}

\begin{tabular}{|c|c|c|}
\hline MEDIUM & COMPONENTS & AMOUNT \\
\hline \multirow[t]{3}{*}{$1 / 2 \mathrm{MS}$ medium } & MS salt & $2.2 \mathrm{~g}$ \\
\hline & Sucrose & $10.0 \mathrm{~g}$ \\
\hline & $\mathrm{ddH} 2 \mathrm{O}$ & Up to $1 \mathrm{~L}$, adjust $\mathrm{pH}$ to 5.7 with $\mathrm{KOH}$ \\
\hline \multirow[t]{3}{*}{ dYT medium for bacteria } & Tryptone & $8.0 \mathrm{~g}$ \\
\hline & Yeast extract & $5.0 \mathrm{~g}$ \\
\hline & $\mathrm{NaCl}$ & $2.5 \mathrm{~g}$ \\
\hline \multirow[t]{6}{*}{ King's B medium for bacteria } & Proteose Pepton & $10.0 \mathrm{~g}$ \\
\hline & K2HPO4 & $1.5 \mathrm{~g}$ \\
\hline & Glycerol & $15.0 \mathrm{~g}$ \\
\hline & $\mathrm{MgSO}_{4}$ & $2 \mathrm{mM}$ \\
\hline & $\mathrm{ddH} 2 \mathrm{O}$ & Up to $1 \mathrm{~L}$, adjust $\mathrm{pH}$ to 7 \\
\hline & \multicolumn{2}{|c|}{ For King's B plates, add bacterial agar to $1.5 \%$. } \\
\hline \multirow[t]{6}{*}{ YEB medium } & Beef extract & $5.0 \mathrm{~g}$ \\
\hline & Yeast extract & $1.0 \mathrm{~g}$ \\
\hline & Peptone & $5.0 \mathrm{~g}$ \\
\hline & Sucrose & $5.0 \mathrm{~g}$ \\
\hline & $\mathrm{MgCl}_{2}$ & $0.5 \mathrm{~g}$ \\
\hline & \multicolumn{2}{|c|}{ For YEB plates, add bacterial agar to $1.5 \%$. } \\
\hline
\end{tabular}

\subsubsection{Antibodies}

\begin{tabular}{l|l|l} 
ANTIBODY & DESCRIPTION & SOURCE \\
\hline Anti-HA (ChIP grade) & $\begin{array}{l}\text { Monoclonal antibody against HA tag } \\
\text { from rabbit }\end{array}$ & Abcam \\
\hline Anti-rabbit & $\begin{array}{l}\text { HRP-conjugated anti rabbit IgG from } \\
\text { goat }\end{array}$ & Life \\
\hline
\end{tabular}


Anti-TGA1

Polyclonal antibody against TGA1 from

Agrisera

rabbit

\subsubsection{Antibiotics}

ANTIBIOTIC

\begin{tabular}{l|c}
\hline Ampicilin & 100 \\
\hline Rifamycin & 50 \\
\hline Spectinomycin & 50 \\
\hline Gentamycin & 25 \\
\hline Kanamycin & 50
\end{tabular}

\subsubsection{Enzymes and kits}

\begin{tabular}{|c|c|}
\hline ENZYME & MANUFACTURER \\
\hline Advantage ${ }^{\circledR} 2$ Polymerase Mix & Clontech \\
\hline BIOTAQ $^{\text {TM }}$ PCR Kit & Bioline \\
\hline Bpil & Thermos Fisher Scientific \\
\hline Bsal & New England Biolabs \\
\hline Bsp1407I & Thermos Scientific \\
\hline Cellulase Onozuka-R10 & Serva \\
\hline Cutsmart & New England Biolabs \\
\hline Eco31I & Thermos Fisher Scientific \\
\hline Macerozyme R-10 & Serva \\
\hline Nhel & Thermos Fisher Scientific \\
\hline NucleoSpin ${ }^{\circledast}$ Gel and PCR clean-up & Macherey-Nagel \\
\hline NucleoSpin ${ }^{\circledast}$ Plasmid & Macherey-Nagel \\
\hline Phusion High-Fidelity Polimerase & Thermos Fisher Scientific \\
\hline Pierce 660nm Protein Assay Reagent & Thermos Fisher Scientific \\
\hline Protein A Agarose Beads & GE Healthcare: rProtein Sepharose TM Fast Flow \\
\hline ReverstAid Reverse Transcriptase & Thermos Fisher Scientific \\
\hline Sall & Fermentas \\
\hline SuperSignal ${ }^{\mathrm{TM}}$ West Femto kit & Thermos Fisher Scientific \\
\hline T4 DNA ligase & Thermos Fisher Scientific \\
\hline T7 endonuclease 1 & New England Biolabs \\
\hline Dual-Luciferase Reporter (DLR ${ }^{\mathrm{TM}}$ ) & Promega \\
\hline
\end{tabular}

FINAL CONCENTRATION MG/L

100
50
50
25
50




\section{Materials and Methods}

\subsection{Methods}

\subsubsection{Standard molecular biology methods}

\subsubsection{Agarose gel electrophoresis}

Products resulting from PCR or plasmid restriction reactions were separated and visualized by agarose gel electrophoresis. Prior to loading, samples were combined with $6 \times$ loading dye and gels were prepared. BioReagent Agarose (Sigma Aldrich) was diluted in 1 X TAE buffer and melted in microwave to a final concentration of $1 \%$. The gel was cast and a comb was inserted to create loading pockets. Once the gel solidified, it was submerged in an electrophoresis tank filled with $1 \times$ TEA. The comb was removed and the DNA-samples were loaded. Separation was conducted under $125 \mathrm{~V}$ for 45 minutes. The gel was incubated for 10 minutes in $\mathrm{EtBr}$ solution to enable its binding to DNA strands prior to fluorescence visualization under UV-light.

\subsubsection{Measurement of DNA and RNA concentrations}

Thermo Scientific ${ }^{\mathrm{TM}}$ NanoDrop 2000 was used to quantify and assess the purity of nucleic acids. $2 \mu \mathrm{l}$ of DNA (plasmid) or RNA was used for measurement at a wave length of $260 \mathrm{~nm}$. The optimal ratio for the sample purity of $D N A$ is $O D_{260} / O D_{280} \approx 1.8$ and for $R N A$ is $O D_{260} / O D_{280} 1.9 \sim 2.0$ and $O_{230} / O D_{260} \approx 2.4$.

\subsubsection{Golden Gate cloning}

The Golden Gate technique is a system for the generation of recombinant plasmids where the vector plasmid and the fragment to be inserted are designed in a way that both molecules carry a restriction site for one specific type Ils endonuclease, e.g. Bpil (Engler et al. 2009). The principle of the method is based on the ability of the enzyme to cleave outside the recognition sequence. DNA ends of the DNA fragment of interest can be designed to be flanked by a type lls restriction site such that digestion of the fragments removes the enzyme recognition sites and generates overhang ends complementary to the overhang ends of the digested vector. Once the wanted DNA is restricted, there is no need to extract or separate products since most of the DNA is restricted. Thus, it is not necessary to separate restriction and ligation processes. Instead both are performed in one restriction-ligation step (Table 1). Plasmids were mixed with PCR products in 1:6 molar ratios, with plasmid amount of approximately $150 \mathrm{ng}$. The reaction was incubated for an hour at $37^{\circ} \mathrm{C}$. Aliquot of $10 \mu \mathrm{L}$ was used for E.coli transformation. 
Table 1 Components of "Golden Gate"

\begin{tabular}{l|c|c} 
COMPONENT & CONCENTRATION & VOLUME / ML \\
\hline pB-CRISPR-AT2S3pGFP & $150 \mathrm{ng} / \mu \mathrm{L}$ & 1 \\
\hline PCR product & $35 \mathrm{ng} / \mu \mathrm{L}$ & 1 \\
\hline ATP & $50 \mathrm{mM}$ & 0.2 \\
\hline Bpil & $10 \mathrm{U} / \mu \mathrm{L}$ & 0.5 \\
\hline T4 DNA ligase & $5 \mathrm{U} / \mu \mathrm{L}$ & 1 \\
\hline Green Buffer & $10 \mathrm{x}$ & 2 \\
\hline $\mathrm{H}_{2} \mathrm{O}$ & & Up to $20 \mu \mathrm{L}$
\end{tabular}

Table 2 Polymerase Chain Reaction program for thermocycler

\begin{tabular}{l|l|l|l|l}
\multirow{2}{*}{ PROGRAM } & STEP & TEMPERATURE $/{ }^{\circ} \mathbf{C}$ & TIME / MIN & NUMBER OF CYCLES \\
\hline \multirow{2}{*}{$\begin{array}{l}\text { PCR program for } \\
\text { cloning }\end{array}$} & Initial denaturation & 98 & 1 & 1 \\
\cline { 2 - 4 } & Denaturation & 98 & 0.25 & \multirow{3}{*}{35} \\
\cline { 2 - 4 } & Annealing & 60 & 0.5 & \\
\cline { 2 - 4 } & Elongation & 72 & $1.5 /$ &
\end{tabular}

\subsubsection{GATEWAYTM cloning}

The plasmids used for Dual-luciferase assay in Arabidopsis protoplast were constructed using the Invitrogen Gateway ${ }^{\mathrm{TM}}$ Technology (Thermo Fisher Scientific, USA) (Katzen 2007). The method is based on the sequence specific recombination system of phage $\lambda$.

In the first step, sequences for recombination are fused to the gene of interest by PCR, enabling the exchange with the donor plasmids cassette using BP clonase II. In the second step, donor plasmid containing region of interest is exchanged with Gateway ${ }^{\mathrm{TM}}$ cassette of the destination vectors pUBQ10GWHAS7 and pBGWL7. For the BP and LR reactions equimolar ratios of PCR fragment or plasmids were mixed with the kit-provided enzyme and incubated for 2 hours. The plasmids were transformed into $E$. coli DH5a.

\subsubsection{Transformation of Escherichia coli}

Transformation of competent Escherichia coli cells ( $\mathrm{DH} 5 \alpha)$ was performed by heat shock method (Hanahan 1983). Cells were incubated for 30 minutes on ice, when a plasmid generated by ligation reaction or Golden Gate cloning was added. Cells were incubated for 30 more minutes on ice followed by heat shock at $42^{\circ} \mathrm{C}$ for 90 seconds. After cooling off on ice for 3 minutes, cells resuspended in 800 $\mu \mathrm{L}$ of $\mathrm{dYT}$ medium were incubated for 45 minutes at $37^{\circ} \mathrm{C}$. Cells were harvested by centrifugation for 


\section{Materials and Methods}

1 minute at $13000 \mathrm{rpm}$ and plated on LB plate containing the selective antibiotic. Plates were incubated overnight at $37^{\circ} \mathrm{C}$.

\subsubsection{Transformation of Agrobacterium tumefaciens by electroporation}

Agrobacterium tumefacies electrocompetent cells (GV3101) were transformed according to (Mattanovich et al. 1989) and incubated on ice for 30 minutes. Meanwhile, cuvettes were washed with distilled water, $70 \%$ ethanol and $100 \%$ ethanol and left to dry. Approximately 100 ng of plasmid was added to the bacteria and incubated for 30 minutes on ice. The Gene Pulser (BioRAD) was set to 2.5 $\mathrm{kV}, 200 \Omega$ and $25 \mu \mathrm{F}$. The cuvette was filled with the cells and electroporation under described condition was conducted for 5 seconds. Immediately $1 \mathrm{~mL}$ of YEB medium was added and cells were incubated for two hours at $29^{\circ} \mathrm{C}$ and $220 \mathrm{rpm}$. Cells were harvested by centrifugation for one minute at $13000 \mathrm{rpm}$ and plated on YEB plates containing selective antibiotics. Plates were incubated for two days at $29^{\circ} \mathrm{C}$.

\subsubsection{Plasmid extraction from E. coli and A. tumefaciens}

Prior to plasmid extraction, bacteria were grown over night at $37^{\circ} \mathrm{C}$ (E. coli) in dYT medium or at $29^{\circ} \mathrm{C}$ (A. tumefacies) in YEB medium with respective antibiotics. We used NucleoSpin ${ }^{\circledR}$ Plasmid (MachereyNagel) for plasmid extraction according to manufacturer manual. Concentration of plasmid was measured by NanoDrop 2000 (peqLab).

\subsubsection{Transformation of Arabidopsis thaliana using the floral dip method}

The Agrobacterium-mediated stable transformation of Arabidopsis was performed as previously described (Clough and Bent 1998). Plants were grown at long day (LD) conditions (16h photoperiod, $22{ }^{\circ} \mathrm{C}, 100-120$ photons $\mathrm{m}^{-2}$ sec $^{-1}$ and $65 \%$ rel. humidity) for approximately four weeks before the first flowers were cut. It took another week for second flowers to grow.

In the meantime, Agrobacterium tumefacies, containing the plasmid of interest, was prepared. Glycerol-stored bacteria were grown on YEB plates on selective antibiotics for two to three days at 29 ${ }^{\circ} \mathrm{C}$ before transferring to liquid media. After two days, $5 \mathrm{~mL}$ aliquot of the first liquid culture was inoculated in $400 \mathrm{~mL}$ of fresh media and grown over night at $29^{\circ} \mathrm{C}$ and $220 \mathrm{rpm}$. The second liquid culture was harvested by centrifugation for 20 minutes at $6000 \mathrm{rpm} / \mathrm{RT}$. The pellet resuspended in 5 \% sucrose solution with $0.02 \%$ Silwet L-77. $\mathrm{OD}_{600}$ of the solution was adjusted to $0.8 \mathrm{~mL}^{-1}$ 


\section{Materials and Methods}

Arabidopsis inflorescences were dipped in the solution for 20 seconds. Plants were covered by a plastic hood and placed in the climate chamber overnight. The following day, hood was removed and plants were grown for a month at LD conditions to allow seed production.

\subsubsection{Extraction of genomic DNA}

Leaf samples from were drilled in $300 \mu \mathrm{L}$ of DNA extraction buffer, followed by centrifugation for 10 min RT/13000. The supernatant was transferred to a new tube and mixed with an equal volume isopropanol and centrifuged at the same conditions as before. The supernatant was removed with a pipette and the pellet was rinsed with $200 \mu \mathrm{L}$ of $70 \%$ ethanol. The supernatant was removed and the pellet was dried at $37{ }^{\circ} \mathrm{C}$. When dry, it was resuspended in $100 \mu \mathrm{L}$ of sterile water and incubated for $10 \mathrm{~min}$ at $65^{\circ} \mathrm{C}$. After centrifugation circa $80 \mu \mathrm{L}$ of the supernatant was transferred to a new tube and stored at $-20^{\circ} \mathrm{C}$.

Table 3 DNA extraction buffer

\begin{tabular}{l|c} 
COMPONENT & FINAL CONCENTRATION \\
\hline Tris-HCl pH7.5 & $200 \mathrm{mM}$ \\
\hline $\mathrm{NaCl}$ & $250 \mathrm{mM}$ \\
\hline EDTA & $25 \mathrm{mM}$ \\
\hline SDS & $0.5 \%(\mathrm{w} / \mathrm{v})$
\end{tabular}

\subsubsection{Generation of CRISPR-Cas9 mutants}

\subsubsection{T1 plants selection}

Selection of T1 of roxy 6 and roxy7 mutants was performed using BASTA treatment. Approximately 100 $\mathrm{mL}$ of sterile seeds were grown in a square pot for one week before three times BASTA treatment was applied for seven days. At least 60 square pots were planted per genotype. After two weeks of growth under LD conditions, surviving plants were transferred to the new pots. Leaf samples were taken from each plant and genomic DNA for genotyping was isolated. For identification of roxy6 roxy7 mutants, leaf samples were collected from individual plants.

Selection of T1 for roxy11-15 and roxy11-15 tga1 tga4 mutant was performed using the blue light filter of a stereo microscope (Zeiss). The glowing seeds which contain the construct were selected and planted on the soil. Plants were grown at LD conditions. Leaf samples were taken from each plant and genomic DNA for genotyping was isolated. Genomic DNA was prepared in pools consisting of five plants and it was subjected to PCR with primers for outermost genes of a cluster ROXY15 and ROXY11. 


\section{Materials and Methods}

The product could be generated only if the deletion occurred, thus the plants which yielded a PCR product of the expected size were allowed to set seeds.

\subsubsection{Identification of plants without the GFP genes}

T2 generation of roxy6, roxy7, roxy11-15 and roxy11-15 tga1 tga4 plants was harvested and the seeds were examined under the blue light filter of a stereo microscope (Zeiss). Approximately 200 nonglowing seeds was selected and planted on pots filled with fresh soil. Plants were grown at LD conditions. Genomic DNA was prepared. Selection of roxy11-15 and roxy11-15 tga1 tga4 mutant was performed as described above by PCR with primers for outermost genes of a cluster ROXY15 and ROXY11. The product could be generated only if the deletion occurred, thus the plants which yielded a PCR product of the expected size were allowed to set seeds. Selection of roxy6 and roxy7 mutants was performed using T7 endonuclease assay.

\subsubsection{T7 endonuclease 1 (T7E1) assay}

Genomic DNA of roxy6 roxy7 transformants that survived the BASTA selection (T1) were examined via the T7E1 assay (Mean et al. 2004). T7 endonuclease 1 recognizes and cleaves heteroduplex DNA that is formed because of heterozygosity of gene alleles. If a DNA fragment consists of two different single strands (e.g. a WT allele and a mutated fragment), it will be cut at the mutated position by T7E1 which results in two fragments. If the DNA fragment consists of identical single-strands, which can be either wild type or mutated, it will not form a heteroduplex and will not be cut.

DNA extracted from leaves was used for PCR reactions with primers for target genes (e.g. ROXY6). PCR efficiency was checked via gel electrophoresis and aliquots of $5 \mu \mathrm{L}$ were subjected to the "Heteroduplex program" (MyCycler, BioRAD). In the meantime, the master mix of T7E1 was prepared (Table 5) and $6 \mu \mathrm{L}$ was added to each tube followed by 60 minutes incubation at $37^{\circ} \mathrm{C}$.

To distinguish between wild type and homozygous plant, PCR products of both are mixed and subject to the "Heteroduplex program" (MyCycler, BioRAD). If examined plant is wild type, there will be no heteroduplexes and therefore just one high running band. If the plant is homozygously mutated, heteroduplexes will be formed and cut by T7E1 and result with three bands on the agarose gel after electrophoresis. 
Table 4 Thermocycles programs for T7E1 assay

\begin{tabular}{l|l|l|l|l} 
PROGRAM & STEP & TEMPERATURE $/{ }^{\circ} \mathrm{C}$ & TIME / MIN & NUMBER OF CYCLES \\
\hline $\begin{array}{l}\text { Heteroduplex } \\
\text { program }\end{array}$ & Denaturation & 95 & 5 & 1 \\
\cline { 2 - 5 } & Annealing & $0,1^{\circ} \mathrm{C} / \mathrm{sec}$ to $15^{\circ} \mathrm{C}$ & $\infty$ & 1 \\
\hline T7E1 assay & Incubation & 37 & 60 & 1
\end{tabular}

Table 5 T7 endonuclease 1 assay mixture for one sample with total volume of $11 \mu \mathrm{L}$ )

\begin{tabular}{l|c|c} 
COMPONENT & CONCENTRATION & VOLUME/ $\mu \mathrm{L}$ \\
\hline T7 endonuclease 1 & $10 \mathrm{U} / \mu \mathrm{L}$ & 0.2 \\
\hline T7E1 buffer & $10 \mathrm{x}$ & 1.1 \\
\hline PCR product & & 5 \\
\hline $\mathrm{ddH}_{2} \mathrm{O}$ & & 4.7
\end{tabular}

\subsubsection{Reporter gene assay in Arabidopsis protoplasts}

\subsubsection{Preparation and transformation of Arabidopsis thaliana mesophyll protoplasts}

Protoplasts were prepared accorind to (Yoo et al. 2007). Plants for protoplast isolation were grown for four to five weeks at SD conditions (12h photoperiod, $22^{\circ} \mathrm{C}, 100$ photons $\mathrm{m}^{-2} \mathrm{sec}^{-1}$ ). 10-20 expanded leaves were cut on abaxial side with scalpel and placed over night in $10 \mathrm{~mL}$ Enzyme solution. The following day, protoplasts were filtrated through a steel net and centrifuged for $2 \mathrm{~min}$ at RT $780 \mathrm{rpm}$ with a soft acceleration and deceleration. Supernatant was removed and pellet was washed and resuspended in W5. Number of protoplasts in suspension was determined under the light microscope using Fuchs-Rosenhalt counting chamber. Suspension was incubated on ice for four hours. After incubation and centrifugation, protoplast were resuspended in $\mathrm{MMg}$ solution to obtain $5 \times 10^{5}$ cells $/ \mathrm{mL}$.

Protoplasts were transformed with $5 \mu \mathrm{g}$ promoter reporter plasmid (DLO1pro::LUC), $5 \mu \mathrm{g}$ effector plasmid (UBQ10::HA, UBQ10::TGA1, UBQ10::TGA2, UBQ10::SARD1) and $1 \mathrm{\mu g}$ control plasmid containing renilla LUCIFERASE gene. In each tube $200 \mu \mathrm{L}$ of protoplast suspension was added and tubes were gently inverted several times. Gene transfer was facilitated by addition of $220 \mu \mathrm{L}$ of PEG solution and incubation for 30 minutes. After the incubation step, $800 \mu \mathrm{L}$ of W5 solution was added and samples were left at RT for 3 minutes. Samples were centrifuged and supernatant was removed and $250 \mu \mathrm{L}$ of WI solution with or without SA was added. Suspensions of protoplasts were incubated over night in the growth chamber. 


\section{Materials and Methods}

Table 6 Buffers for protoplast isolation and transformation

\begin{tabular}{l|l|l|l}
\multicolumn{2}{l}{ ENZYME SOLUTION } & MMg SOLUTION & $0.4 \mathrm{M}$ \\
\hline Celullase R10 & $1.25 \%$ & Mannitol & $15 \mathrm{mM}$ \\
\hline Macerozyme R10 & $0.3 \%$ & $\mathrm{MgCl}_{2}$ & $4 \mathrm{mM}$ \\
\hline Mannitol & $0.4 \mathrm{M}$ & MES & \multicolumn{2}{l}{} \\
\hline $\mathrm{KCl}$ & $20 \mathrm{mM}$ & PEG SOLUTION & $40 \%$ \\
\hline $\mathrm{MES}$ & $20 \mathrm{mM}$ & PEG 4000 & $100 \mathrm{mM}$ \\
\hline $\mathrm{CaCl}$ & & $\mathrm{CaCl}_{2}$ & $0.2 \mathrm{M}$ \\
\hline W5 SOLUTION & $10 \mathrm{mM}$ & Mannitol $^{2}$ & \multicolumn{2}{l}{} \\
\hline $\mathrm{NaCl}$ & $154 \mathrm{mM}$ & WI SOLUTION & $0.5 \mathrm{M}$ \\
\hline $\mathrm{CaCl}$ & & Mannitol & $4 \mathrm{mM}$ \\
\hline $\mathrm{KCl}$ & $125 \mathrm{mM}$ & MES & $20 \mathrm{mM}$
\end{tabular}

\subsubsection{Luciferase assay}

Promoter luminescence was performed using Dual-Luciferase Reporter (DLR ${ }^{\mathrm{TM}}$ ) assay system from Promega following user's instructions. Luminescence was measured and ratio of fLUC (reporter plasmid) and rLUC (control plasmid) was calculated.

\subsubsection{Plant growth and treatment}

\subsubsection{Surface sterilization of plant seeds}

Seeds were sterilized in a desiccator with a mixture of $50 \mathrm{~mL}$ sodium hypochlorite $(\mathrm{NaClO})$ solution and $2.5 \mathrm{~mL}$ of $32 \%$ hydrochloric acid. The desiccator was closed under a weak vacuum for two hours. Sterilized seeds were left to dry on the air for one to two hours.

\subsubsection{Plant growth conditions}

Individual plants were grown in round pots for the SA treatment experiments at short day (SD) conditions (12h photoperiod, $22{ }^{\circ} \mathrm{C}, 130-150$ photons $\mathrm{m}^{-2} \mathrm{sec}^{-1}$ and $65 \%$ rel. humidity) for approximately four weeks. Four days before the SA experiments, plants were transferred to a smaller growth chamber with the same conditions as in the first growth chamber. 


\section{Materials and Methods}

\subsubsection{Salicylic acid treatment}

$100 \mathrm{~mL}$ of $1 \mathrm{mM}$ solution of sodium salicylate was prepared freshly for each experiment. All the experiments were conducted 1 hour after the beginning of the light period. First, the water was sprayed until leaves were equally moisture and plants were briefly covered with a lid while SA was sprayed in the same way on the other plants. The lid was removed and the plants were left for 8 and 24 hours following the sample collection for RNA isolation.

\subsubsection{GUS staining}

GUS reporter gene constructs were used to localize target gene promoter activity. Transgenic plants were grown on SD conditions for three to four weeks prior to SAR experiment to induce reporter gene expression and accumulation of the $\beta$-glucuronidase enzyme. Tree infiltrated leaves were harvested into $5 \mathrm{~mL}$ reaction tubes and $5 \mathrm{~mL}$ GUS fixative solution was added. The tubes were rolled for $30 \mathrm{~min}$ at RT and the fixative was removed. The samples were washed two times with $50 \mathrm{~mm}$ sodium phosphate buffer ( $\mathrm{pH} 7.0$ ) for $20 \mathrm{~min}$ at RT prior to vacuum infiltration of freshly prepared GUS staining solution. Samples were infiltrated for three minutes three times. The tubes were incubated $\mathrm{ON}$ at $37^{\circ} \mathrm{C}$ and the staining solution was exchanged for $100 \% \mathrm{EtOH}$.

Table 7 GUS fixative solution

\begin{tabular}{l|c} 
COMPONENT & CONCENTRATION \\
\hline formaldehyde & $0.3 \%$ \\
\hline mannitol & $0.3 \mathrm{M}$ \\
\hline sodium phosphate buffer $\mathrm{pH} 7.0$ & $50 \mathrm{mM}$
\end{tabular}

Table 8 GUS staining solution

\begin{tabular}{l|c} 
COMPONENT & CONCENTRATION \\
\hline $\mathrm{K}_{3}\left[\mathrm{Fe}(\mathrm{III})(\mathrm{CN})_{6}\right]$ & $0.5 \mathrm{mM}$ \\
\hline $\mathrm{K}_{4}\left[\mathrm{Fe}(\mathrm{II})(\mathrm{CN})_{6}\right]$ & $0.5 \mathrm{mM}$ \\
\hline $\mathrm{EDTA}$ & $10 \mathrm{mM}$ \\
\hline Triton X-100 & $0.1 \%$ \\
\hline sodium phosphate buffer $\mathrm{pH} 7.0$ & $50 \mathrm{mM}$ \\
\hline
\end{tabular}

Add $2.5 \mathrm{mM}$ X-Gluc freshly prepared in $3 \mathrm{~mL}$ DMF 


\section{Materials and Methods}

\subsubsection{Pathogen assays}

\subsubsection{Plant growth conditions for Pseudomonas syringae infection}

Four plants were grown in square pots for the infection experiments at SD conditions (12h photoperiod, $22^{\circ} \mathrm{C}, 130-150$ photons $\mathrm{m}^{-2} \mathrm{sec}^{-1}$ and $65 \%$ relative humidity) for four to five weeks.

\subsubsection{Cultivation of Pseudomonas syringae}

Bacteria were grown on King's B plates containing appropriate antibiotics, Psm ES4326 with Rifampicin and Pst DC3000 $\triangle$ avrPto/PtoB with Rifampicin and Kanamycin. After 3 days at $29^{\circ} \mathrm{C}$ incubator, the plates were stored at $4^{\circ} \mathrm{C}$. Every three weeks, bacteria were transferred to the fresh plates. Prior to the infection, bacteria were grown in the liquid King's B media and in $29^{\circ} \mathrm{C}$ shaker.

\subsubsection{Infection with Pseudomonas syringae}

Plants were watered in the early morning and covered with the lid for 2 hours. In the meantime, bacteria, grown overnight in King's B medium with appropriate antibiotics, were centrifuged 10 minutes at $\mathrm{RT} / 4000 \mathrm{rpm}$ and washed 3 times with $10 \mathrm{mM}$ magnesium chloride $\left(\mathrm{MgCl}_{2}\right)$. The $\mathrm{OD}_{600}$ was measured and $\mathrm{MgCl}_{2}$ dilution of $\mathrm{OD}_{600} 0.0001$ for virulent strain or 0.002 for avirulent strain were prepared. Three to five leaves of each plant were syringe infiltrated with the bacteria and left for 3 days.

\subsubsection{Systemic Acquired Resistance (SAR) experiment}

Individual plants were grown in round pots for the SAR experiment at short day (SD) conditions for approximately five weeks. Three older leaves were infiltrated with Psm solution of $\mathrm{OD}_{600} 0.005$ and left for two days. Younger upper leaves were used for secondary Psm infection with $\mathrm{OD}_{600} 0.005$ and samples were harvested eight hours post infection. As a control, primary and secondary leaves were also infiltrated with $10 \mathrm{mM} \mathrm{MgCl}_{2}$ or left untreated, leading to nine different treatments in a course of an experiment.

\subsubsection{Bacterial growth assays}

In order to determine bacterial growth on the plants, three discs from three infected leaves of a single plant were diluted in $0.2 \mathrm{~mL}$ of $10 \mathrm{mM} \mathrm{MgCl}_{2}$ in $1.5 \mathrm{~mL}$ tube with a metal bead. Samples were crushed 
and dilutions from 1:1 to 1:10000 were prepared. Two replicates of $15-20 \mu \mathrm{L}$ of each sample dilution were plated on King's B plates and incubated $2-3$ days at $29^{\circ} \mathrm{C}$. Colonies were count from all samples, each genotype having at least six biological replicates. Colony forming unit (CFU) was calculated as following:

$$
\begin{array}{cl}
\mathrm{CFU}=\frac{\mathrm{N}(\mathrm{CFU}) * \text { dilution factor }}{\mathrm{A} * \mathrm{~V}} & \mathrm{~N}-\text { Number of colonies } \\
& \mathrm{A}-\text { Area of } 9 \text { leaf discs } \\
& \mathrm{V}-\text { Volume }
\end{array}
$$




\section{Materials and Methods}

\subsubsection{Transcript analysis}

\subsubsection{RNA extraction}

Three leaves of an individual plant were collected in screw-top $2 \mathrm{~mL}$ tubes containing 2 metal beads, shock frozen in liquid nitrogen and stored at $-80^{\circ} \mathrm{C}$. Samples were then ground in Tissuelyser (Retsch) with the amplitude of $20 \mathrm{~Hz}$ for 1 minute. RNA was isolated using the TRIZOL method (Chomczynski 1993). $1.4 \mathrm{~mL}$ of TRIZOL buffer was added to the ground tissue following 5 minutes of vigorously shaking. After addition of $0.25 \mathrm{~mL}$ of chloroform, samples were shaken for 10 more minutes following a centrifugation for 40 minutes at $4^{\circ} \mathrm{C} / 12000 \mathrm{rpm}$. Approximately $1 \mathrm{~mL}$ of supernatant was transferred to new tube containing $0.4 \mathrm{~mL}$ high salt buffer and $0.4 \mathrm{~mL}$ isopropanol and gently mixed by inverting several times and left still for $10 \mathrm{~min}$. The samples were centrifuged for 30 minutes at the same conditions. The supernatant was decanted and the pellet was washed two times with $0.5 \mathrm{~mL} 70 \% \mathrm{EtOH}$. Samples were centrifuges for 5 minutes under the same conditions and the supernatant was removed by a pipette. The pellet was left to dry for a half an hour and then diluted in 50 to $200 \mu \mathrm{L}$ water. The solution was incubated for $10-20$ minutes at $65^{\circ} \mathrm{C}$ and centrifuged for 5 minutes at RT/12000 rpm. Samples were stored at $-20^{\circ} \mathrm{C}$.

Table 9 High salt buffer receipt

\begin{tabular}{l|c|c} 
COMPONENT & FINAL CONCENTRATION & VOLUME \\
\hline $\mathrm{Nacl}$ & $1.2 \mathrm{M}$ & $3.51 \mathrm{~g}$ \\
\hline Tri-sodium-citrate & $0.8 \mathrm{M}$ & $11.76 \mathrm{~g}$ \\
\hline $\mathrm{ddH}_{2} \mathrm{O}$ & & Up to $50 \mathrm{~mL}$
\end{tabular}

Table 10 TRIZOL buffer components

\begin{tabular}{l|c|c} 
COMPONENT & FINAL CONCENTRATION & VOLUME \\
\hline $\begin{array}{l}\text { Phenol saturated with 1M Na-acetate, } \\
\text { pH 5.2 }\end{array}$ & $380 \mathrm{~mL} / \mathrm{L}$ & $190 \mathrm{~mL}$ \\
\hline Guanidine thiocyanate & $0.8 \mathrm{M}$ & $47.26 \mathrm{~g}$ \\
\hline Ammonium thiocyanate & $0.4 \mathrm{M}$ & $15.22 \mathrm{~g}$ \\
\hline Na-acetate 3M, pH 5.2 & $0.1 \mathrm{M}$ & $16.7 \mathrm{~mL}$ \\
\hline Glycine & $5 \%$ & $25 \mathrm{~mL}$ \\
\hline dd $\mathrm{H}_{2} \mathrm{O}$ & & $\mathrm{Up} \mathrm{to} 500 \mathrm{~mL}$
\end{tabular}

\subsubsection{2 cDNA synthesis}

The amount corresponding to $1 \mu \mathrm{g}$ of RNA was pipetted in each tube following the addition of water up to $8 \mu \mathrm{L}$. The samples were incubated for 30 minutes at $37^{\circ} \mathrm{C}$ with $1 \mu \mathrm{L}$ of DNase I together with $1 \mu \mathrm{L}$ of 10x DNAse I buffer to degrade all the residual DNA from the RNA isolation. DNase I was deactivated 


\section{Materials and Methods}

by addition of $1 \mu \mathrm{L}$ of $25 \mathrm{mM}$ EDTA and subjected to 10 minutes incubation at $65^{\circ} \mathrm{C} .1 .2 \mu \mathrm{L}$ of Master Mix 2 was added to each tube and subjected to Program 70. Later, samples were incubated for 10 minues at $70^{\circ} \mathrm{C}$ with 20 pmol oligo-dT which binds to poli-A tail of mRNA and enriches during the reverse transcription. Finally, reverse transcriptase, $20 \mathrm{nmol} d N T P s, 4 \mu \mathrm{L} 5 \times \mathrm{RT}$-buffer and water were added for 70 minute incubation at $42^{\circ} \mathrm{C}$ and 10 minutes at $70^{\circ} \mathrm{C}$. cDNA was then diluted in 1:10 ratio in new $1.5 \mathrm{~mL}$ tubes with water.

Table 11 Reaction mix for cDNA synthesis using Reverse Transcriptase

\begin{tabular}{l|c|c} 
COMPONENT & VOLUME & PROGRAM \\
\hline RNA & $1 \mu \mathrm{g}$ & \multirow{2}{*}{$37^{\circ} \mathrm{C}, 30 \mathrm{~min}$} \\
\hline $10 \mathrm{x}$ DNase buffer & $1 \mu \mathrm{L}$ & \\
\hline DNase & $1 \mu \mathrm{L}$ & \\
\hline $\mathrm{H}_{2} \mathrm{O}$ & Up to $10 \mu \mathrm{L}$ & $65^{\circ} \mathrm{C}, 10 \mathrm{~min}$ \\
\hline $25 \mathrm{mM}$ EDTA & $1 \mu \mathrm{L}$ & $70^{\circ} \mathrm{C}, 10 \mathrm{~min}$ \\
\hline $100 \mu$ M oligo-dT & $0.2 \mu \mathrm{L}$ & \\
\hline $5 \times$ RT-buffer & $4 \mu \mathrm{L}$ & $42^{\circ} \mathrm{C}, 70 \mathrm{~min}$ \\
\hline $10 \mathrm{mM}$ dNTPs & $2 \mu \mathrm{L}$ & $70^{\circ} \mathrm{C}, 10 \mathrm{~min}$ \\
\hline Reverse Transcriptase & $0.2 \mu \mathrm{L}$ & \\
\hline ddH $\mathrm{H}_{2} \mathrm{O}$ & Up to $20 \mu \mathrm{L}$ &
\end{tabular}

\subsubsection{Quantitative real time-PCR (qRT-PCR)}

qRT-PCR was performed using MyiQ ${ }^{\text {TM }}$ Real-Time PCR Detection Systems (Bio-Rad, USA). For each reaction, $1 \mu \mathrm{l}$ of 1:10 diluted cDNA was incubated in $25 \mu \mathrm{L}$ reaction with $0.4 \mu \mathrm{M}$ primers, $2 \mathrm{mM} \mathrm{MgCl}_{2}$, $100 \mu \mathrm{M}$ dNTPs, $1 \mu \mathrm{L}$ of $10 \times \mathrm{NH}_{4}$-buffer, 1:100000 dilution of SYBR Green solution, $10 \mathrm{nM}$ fluoresceine, $0.25 \mathrm{U}$ BIOTAQ polymerase and $17.2 \mu \mathrm{L}$ of water. Reaction started with 6 minutes of denaturation, followed by 35 cycles of $20 \mathrm{~s}$ at $95^{\circ} \mathrm{C}, 20 \mathrm{~s}$ at $55^{\circ} \mathrm{C}$ and $40 \mathrm{~s}$ at $72^{\circ} \mathrm{C}$.

Table 12 Reaction mix for qRT-PCR with BIOTAQ DNA Polymerase

\begin{tabular}{l|c} 
COMPONENT & VOLUME \\
\hline $10 \mathrm{x} \mathrm{NH}_{4}$ reaction buffer & $2.5 \mu \mathrm{L}$ \\
\hline $\mathrm{MgCl}_{2} 25 \mathrm{mM}$ & $1 \mu \mathrm{L}$ \\
\hline $\mathrm{dNTPs} 10 \mathrm{mM}$ & $0.25 \mu \mathrm{L}$ \\
\hline $\mathrm{RT}$ - primer pair, each 4 mM & $2.5 \mu \mathrm{l}$ \\
\hline Sybr Green, 1/1000 & $0.25 \mu \mathrm{l}$ \\
\hline Fluorescein 1 $\mu \mathrm{M}$ & $0.25 \mu \mathrm{l}$ \\
\hline BIOTAQ DNA Polymerase & $0.05 \mu \mathrm{l}$ \\
\hline cDNA template & $1 \mu \mathrm{l}$ \\
\hline ddH $\mathrm{O}_{2} \mathrm{O}$ & Up to $25 \mu \mathrm{L}$
\end{tabular}




\section{Materials and Methods}

For the melting curve analysis, samples were incubated for 4 min at $72^{\circ} \mathrm{C}$. Transcripts were normalized to housekeeping gene UBQ5 and the relative gene expression was calculated with a software iQ5 provided by Bio-Rad using $2^{\text {-CT(gene of interest)-CT(reference gene)] }}$ method (Livak and Schmittgen 2001).

Table 13 Program for qRT-PCR with BIOTAQ DNA Polymerase

\begin{tabular}{l|r|c}
\multirow{2}{*}{ CYCLE STEP } & TEMPERATURE AND DURATION & CYCLES \\
\hline Final extension & $72^{\circ} \mathrm{C}, 4 \mathrm{~min}$ & 1 \\
\hline Denaturation & $95^{\circ} \mathrm{C}, 20 \mathrm{sec}$ & 39 \\
\hline Annealing & $55^{\circ} \mathrm{C}, 20 \mathrm{sec}$ & \\
\hline Extension & $72^{\circ} \mathrm{C}, 40 \mathrm{sec}$ & 1 \\
\hline Final extension & $72^{\circ} \mathrm{C}, 4 \mathrm{~min}$ & 1 \\
\hline Generation of melting curve & $95^{\circ} \mathrm{C}, 1 \mathrm{~min}$ & 1 \\
\hline & $55^{\circ} \mathrm{C}, 1 \mathrm{~min}$ & 81
\end{tabular}

\subsubsection{RNA sequencing data analysis}

Individual sid2 and sid2 tga1 tga4 plants were grown on soil for four weeks before the treatment with $\mathrm{SA}$, as described above. Experiment was repeated four times with 5 samples per genotype and treatment. RNA was isolated using Trizol method and cDNA synthetized in order to confirm SA induction in the SA treated samples by qRT-PCR. 5 samples of the same treatment and genotype of one experiment were pooled to serve as one sample for RNA. Altogether, four independent experiments resulted in 32 samples for RNA sequencing.

RNA sequencing was performed by Transcriptome and Genome Analysis Laboratory (TAL). Reads of each sample were aligned against Arabidopsis thaliana reference sequence (TAIR10) to obtain gene counts. Statistical analysis was performed using RobiNA software (Lohse et al. 2012) with gene counts serving as an input data set. The program interface allows the user to choose groups for comparison and calculates fold change values and corresponding $p$-values. As a control of data distribution, PCA (Principal Component Analysis) and hierarchical clustering of all samples was generated. Generally, samples of the same genotype and treatment have more similar transcriptome datasets and tend to cluster together. DEG (Differentially expressed genes) were obtained using a fold change (log FC $>1$ or $<-1, p<0.05)$.

Venn diagrams were generated using BioVenn web application (Hulsen et al. 2008). Gene Ontology (GO) Enrichment analysis was performed using Gene Ontology Consortium web interface (The Gene Ontology Resource: 20 years and still GOing strong 2019; Ashburner et al. 2000). Groups of genes were sorted according to the biological processes terms using PANTHER (Protein Analysis Through 


\section{Materials and Methods}

Evolutionary Relationships, http://pantherdb.org) (Mi et al. 2019) and percentage of genes was calculated relative to a number of genes in input data set.

Promoter enrichment analysis was conducted using Motif Mapper software (version 5.2.4.01). It calculates enrichment of the motifs in input data set relative to 1000 randomly composed and equally sized motifs from reference data set (Berendzen et al. 2012).

\subsubsection{Protein analysis}

\subsubsection{Extraction of total proteins}

Leaf of root tissue was collected and frozen in liquid nitrogen. To approximately $200 \mathrm{mg}$ of ground material $200 \mu \mathrm{L}$ of urea buffer (4M Urea, $16.6 \%$ glycerol, $5 \%$ SDS, $0.5 \% \beta$-mercaptoethanol) was added and mixed for 10 minutes at $65^{\circ} \mathrm{C}$. Solution was then centrifuged for 15 minutes at RT/13000 rpm and supernatant as used for further protein analysis.

Table 14 Urea buffer form total protein extraction

\begin{tabular}{l|c} 
COMPONENT & FINAL CONCENTRATION \\
\hline Urea & $4 \mathrm{M}$ \\
\hline Glycerol & $16.6 \%(\mathrm{v} / \mathrm{v})$ \\
\hline SDS & $5 \%(\mathrm{w} / \mathrm{v})$ \\
\hline B-mercaptoethanol & $0.05 \%(\mathrm{w} / \mathrm{v})$
\end{tabular}

\subsubsection{Protein concentration measurement}

Protein concentrations were determined using $0.05 \mathrm{~g} / \mathrm{mL}$ lonic Detergent Compatibility Reagent (IDCR) solution in Pierce 660nm Protein Assay Reagent. $1 \mu \mathrm{L}$ of protein extract was incubated 5 minutes at room temperature with $150 \mu \mathrm{L}$ of the reagent solution. $\mathrm{OD}_{660}$ was measured using Synergy $H T$ plate reader (BioTek) and concentration were determined using standard curve derived from $0 \mu \mathrm{L}, 3 \mu \mathrm{L}, 6$ $\mu \mathrm{L}, 12 \mu \mathrm{L}$ and $18 \mu \mathrm{L}$ of $0.5 \mathrm{mM}$ Bovine serum albumin (BSA).

\subsubsection{SDS-PAGE}

Separation of proteins was performed using SDS-PAGE (sodium dodecyl sulfate-polyacrylamide gel electrophoresis) method described by (Weber et al. 1977). $15 \mu \mathrm{g}$ of protein extracts were loaded to the stacking gel ( $4 \%$ acrylamide, $0.125 \mathrm{M}$ Tris $\mathrm{pH} 6.8,0.1 \%$ SDS, $0.1 \%$ APS, $0.1 \%$ TEMED) and the electrophoresis was performed at $150 \mathrm{~V}$ for 1 hour and 30 minutes on $12 \%$ separating gel (12\% 


\section{Materials and Methods}

acrylamide, $0.375 \mathrm{M}$ Tris pH 8.8, 0.1 \% SDS, $0.1 \%$ APS, $0.1 \%$ TEMED). Protein size was estimated in comparison to a prestained ladder $(6 \mu \mathrm{L})$.

Table 15 Solutions for SDS-PAGE gel and protein separation via electrophoresis

\begin{tabular}{l|c|c|c} 
COMPONENT & $\mathbf{1 2} \%$ SEPARATING GEL & $\mathbf{4} \%$ STACKING GEL & RUNNING BUFFER (1 L) \\
\hline Acrylamide $30 \%$ & $8 \mathrm{~mL}$ & $1,34 \mathrm{~mL}$ & \\
\hline Tris-HCl $1.5 \mathrm{M}, \mathrm{pH} 8.8$ & $5 \mathrm{~mL}$ & $/$ & \\
\hline Tris-HCl $1 \mathrm{M}, \mathrm{pH} 6.8$ & $/$ & $1,25 \mathrm{~mL}$ & \\
\hline SDS $10 \%$ & $0,2 \mathrm{~mL}$ & $0,1 \mathrm{~mL}$ & \\
\hline APS $10 \%$ & $0,2 \mathrm{~mL}$ & $0,1 \mathrm{~mL}$ & \\
\hline TEMED & $0,02 \mathrm{~mL}$ & $0,01 \mathrm{~mL}$ & $0.025 \mathrm{M}$ \\
\hline $\mathrm{H}_{2} \mathrm{O}$ & $6,6 \mathrm{~mL}$ & $7,2 \mathrm{~mL}$ & $0.2 \mathrm{M}$ \\
\hline TRIS & & & $2 \%$
\end{tabular}

\subsubsection{Immunoblot analysis}

Proteins separated by SDS-PAGE were transferred to PVDF (Polyvinylidene difluoride, Roti $^{\circledR}$-PVDF, Roth) membrane using semi-dry blot method at currency of $0.1 \mathrm{~mA} / \mathrm{cm}^{2}$ for 1 hour 30 minutes. The membrane was then blocked with $5 \%(w / v)$ non-fat milk in TBST for 2 hours following the immunoblot with rabbit $\alpha$-TGA1 antibody (Agrisera, 1:1000 dilution). Secondary antibody against rabbit (Life, 1:8000 dilution) was incubated for 2 hours. Antibodies were diluted in $5 \%(w / v)$ non-fat milk in TBST. Chemiluminescence method was used for antigen detection with a SuperSignal ${ }^{\mathrm{TM}}$ West Femto Maximum Sensitivity Substrate kit (Thermo scientific) according to the manufacturer's protocol and the luminescence was detected in a chemocam (Intas).

Table 16 Solutions for protein transfer and detection

\begin{tabular}{l|c|c} 
COMPONENT & TRANSFER BUFFER & TBST (PH 7.5) \\
\hline TRIS & $0.05 \mathrm{M}$ & $0.02 \mathrm{M}$ \\
\hline Glycin & $0.04 \mathrm{M}$ & \\
\hline SDS & $2 \%$ & \\
\hline Methanol & $20 \%$ & $0.14 \mathrm{M}$ \\
\hline $\mathrm{NaCl}$ & & $1 \%$
\end{tabular}




\section{Results}

\subsection{RNA sequencing data revealed that TGA1/TGA4 activate a subset of genes downstream of SA}

\subsubsection{SA treatment induced transcriptional changes in sid2 and sid2 tga1 tga4 mutant}

In order to identify TGA1/TGA4 target genes downstream of salicylic acid (SA), transcriptome analysis was performed with RNA from leaves of SA-treated sid2 and sid2 tga1 tga4 mutant plants. We decided to use SA biosynthesis mutant sid2 to avoid the influence of TGA1/TGA4 on biosynthesis of endogenous SA (Wildermuth et al. 2001; Sun et al. 2018). Four-week-old plants were sprayed either with water, as a control treatment, or 1mM SA. Eight- and twentyfour-hours post treatment three leaves of five individual plants were collected and total RNA was isolated. The experiment was repeated four times with batches of individually grown plants. The RNA from five plants of the same genotype and treatment from each experiment was combined to serve as a single sample for RNA sequencing. Samples were sequenced by Transcriptome and Genome Analysis Laboratory (TAL) (University of Göttingen).

Principal Component Analysis was performed as a control of data distribution. Generally, samples of the same genotype and treatment have more similar transcriptome datasets and tend to cluster together. The SA-treated samples of both genotypes showed clear separation from water-treated ones, indicating that the SA treatment triggered transcriptional changes. In both time points clusters of the mock-treated samples of different genotypes showed stronger separation than the SA-treated ones. This would indicate that our samples differed more after the mock treatment than after the SA treatment. Eight hours after treatment all the clusters were separated while twenty-hours after treatment there was an overlap of SAtreated samples of sid2 and mock treated samples of sid2 tga1 tga4 (Figure 3-pink triangles and yellow squares). Eight hours post SA treatment samples of sid2 and sid2 tga1 tga4 clustered very close together, indicating similar responses in both genotypes (Figure 3). 


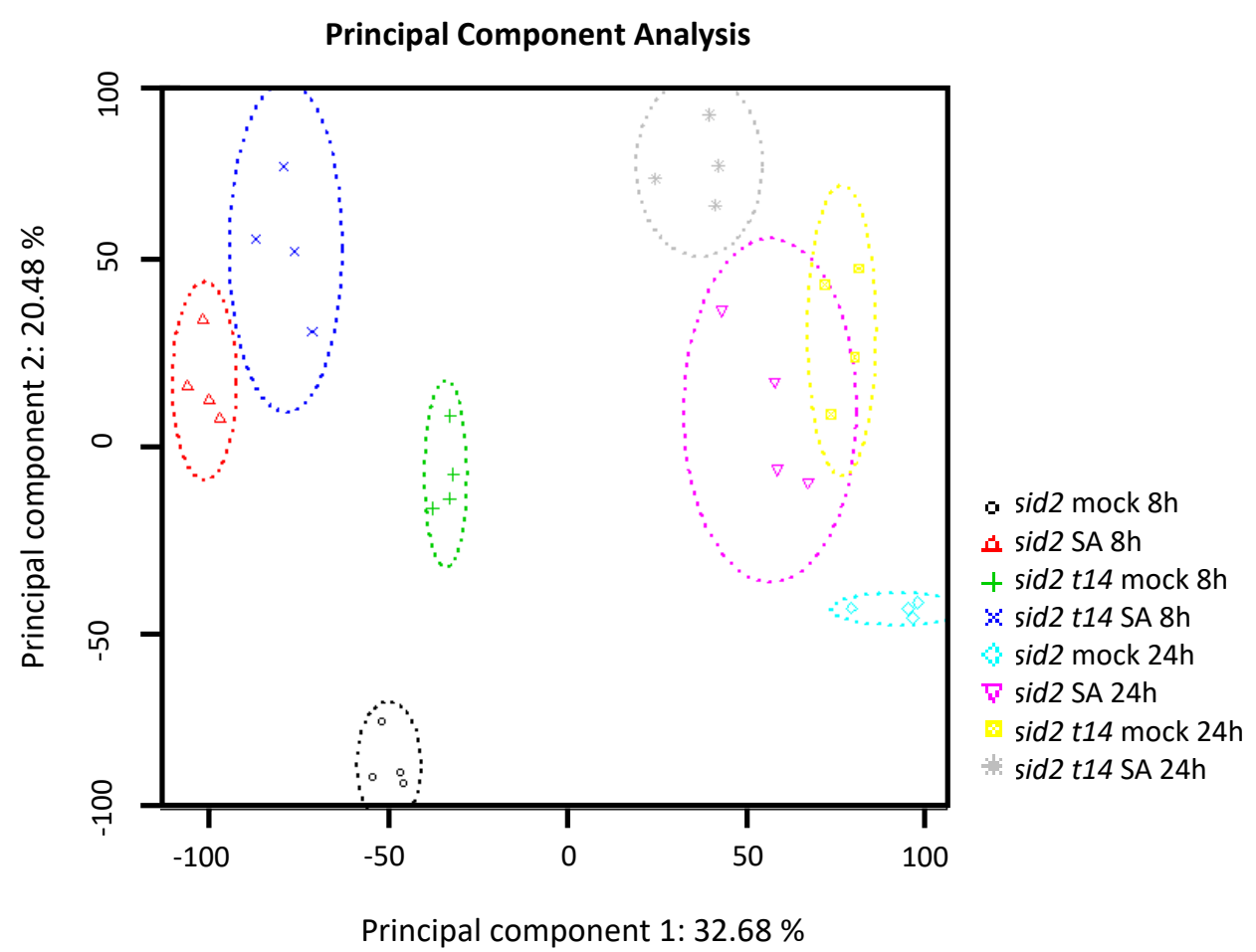

Figure 3 Transcriptional differences between sid 2 and sid 2 tga 1 tga 4 after mock and SA treatment.

PCA (Principal Component Analysis) of sid2 and sid 2 tga 1 tga 4 samples treated either with water or $1 \mathrm{mM}$ SA for 8 and 24 hours. Symbols represent individual biological replicates. Statistical analysis was performed using RobiNA software (Lohse et al. 2012).

Statistical analysis for determination of DEG (Differentially Expressed Genes) (fold change ( $\log 2 \mathrm{FC}>1$ or $\log 2 \mathrm{FC} \leq-1), \mathrm{p}<0.05$ ) was conducted using RobiNA software (Lohse et al., 2012). The number of up- and down-regulated genes after the SA treatment is listed in Table 17. Approximately $50 \%$ of sid 2 SA-inducible gene was also inducible in sid 2 tga 2 tga4 (fold change ( $\log 2 \mathrm{FC}>1), \mathrm{p}<0.05)$ after eight and twenty-four hours of treatment.

Table 17 Number of differently expressed genes is sid2 and sid2 tga1 tga4 after eight and twenty-four hours of SA treatment.

\begin{tabular}{l|l|l|l}
\multirow{2}{*}{ Genotype } & Time after treatment & SA-inducible genes & SA-repressed genes \\
\hline \multirow{2}{*}{ sid2 tga1 tga4 } & $8 \mathrm{~h}$ & 2145 & 1917 \\
\cline { 2 - 4 } & $24 \mathrm{~h}$ & 1218 & 336 \\
\hline Both genotypes & $8 \mathrm{~h}$ & 1542 & 1173 \\
\cline { 2 - 4 } & $24 \mathrm{~h}$ & 1081 & 445 \\
\hline & $8 \mathrm{~h}$ & 1116 & 898 \\
\cline { 2 - 4 } & $24 \mathrm{~h}$ & 582 & 120
\end{tabular}




\subsubsection{A group of 207 SA-inducible genes was TGA1/TGA4-dependent}

Salicylic acid causes transcriptional reprograming of the plant, primarily induction of PAHTOGENESIS RELATED (PR) genes involved in defense against biotrophic pathogens. After the SA treatment, $2145(8 \mathrm{~h})$ and $1218(24 \mathrm{~h})$ genes were induced in sid2 mutant (fold change $(\log 2 \mathrm{FC}>1), \mathrm{p}<0.05)$. In sid2 tga1 tga4 mutant 1542 ( $8 \mathrm{~h}$ ) and 1081 (24 h) genes were induced (fold change (log2 FC >1), $\mathrm{p}<0.05)$.

In order to identify TGA1/TGA4-dependent genes which are SA-inducible, we compared a group of SA-inducible genes of sid2 with the genes that were less expressed in sid2 tga1 tga4 mutant after the SA treatment. Not to exclude the possibility of TGA1/TGA4 being negative regulators of transcription, we also included genes that were higher expressed in sid2 tga1 tga4 than in sid2 after SA treatment. The same comparison was performed for both time points (Table 18) resulting in Venn diagrams for both time points (Error! Reference source not $f$ ound.A and Supplementary Figure 1A).

Table 18 DEG between sid2 and sid2 tga1 tga4 mutant after mock or SA treatment for eight and twenty-four hours.

\begin{tabular}{|c|c|c|c|c|}
\hline \multirow[b]{2}{*}{ Comparison } & \multicolumn{2}{|l|}{$8 \mathrm{~h}$} & \multicolumn{2}{|l|}{$24 h$} \\
\hline & Number of genes & SA inducible in sid2 & Number of genes & SA inducible in sid2 \\
\hline $\operatorname{sid} 2>\operatorname{sid} 2$ tgal tga4 (SA) & 554 & 207 & 1042 & 239 \\
\hline $\operatorname{sid} 2<\operatorname{sid} 2 \operatorname{tga} 1 \operatorname{tga} 4(\mathrm{SA})$ & 1044 & 144 & 1400 & 193 \\
\hline
\end{tabular}

Then, we used all the subsets of genes from the Venn diagram and analyzed Gene Ontology enrichment for biological processes (Error! Reference source not found.A and Supplementary $F$ igure 1A). For this we used an online tool (http://geneontology.org/). The provided algorithm calculates enrichment of the uploaded list of genes as compared to the chosen background set. As a background set one can use either whole Arabidopsis genome or a chosen group of genes. Because SA treatment causes transcriptional changes which are already enriched in GO terms for defense, we could not use Arabidopsis genome as a background set (Supplementary Figure 2). Instead, we used a group of SA-inducible genes in sid2 in the respective time points. This enabled us to see if this specific group is even more enriched in defense responses than all the SA-induced genes. 
The group of 207 ( 8 h) but not the group of 239 (24 h) genes was enriched in GO (Gene Ontology) biological processes terms "defense response against bacterium" and "biotic stress" (Error! Reference source not found.B). The group of genes which were less expressed $i$ $\mathrm{n}$ mutant but not SA-inducible in sid 2 was highly enriched in GO biological processes "response to auxin", "mRNA transcription" and "response to light stimulus" (Error! Reference source not found.D \& Supplementary Figure 1B). This was consistent in both time points and fit to the published data connecting TGA1/TGA4 with auxin regulation and hyponastic growth (Li et al, 2018). There was an overlap of 177 genes which were SA-inducible in sid 2 and more expressed in the triple mutant background. This group was enriched in "hormone metabolic processes", "regulation of mRNA biosynthesis" and "nitrogen compound metabolism" when the 2145 SAinducible genes were used as a background set (Error! Reference source not found.C). The group of genes which were more expressed in the triple mutant but not SA-inducible in sid2 were highly enriched in GO biological processes "response to wounding", "water deprivation" and "osmotic stress" in both time points (Error! Reference source not found.E \& Supplementary Figure 1C).

The only group enriched in GO terms "defense response against bacterium" and "biotic stress" was a group of $207 \mathrm{SA}$-inducible genes that were also less expressed in sid 2 tga 1 tga4 after SA treatment. Since we were interested in TGA1/TGA4 regulated genes downstream of SA, we used this data set for further analysis.

The data set from samples collected twenty-four hours after SA treatment showed enrichment in circadian clock regulated genes (Supplementary Figure 1B - "photosynthesis, light harvesting"). We wanted to exclude possible pleiotropic effects of circadian rhythm on the gene expression, therefore we did not use this timepoint in the following experiments. 
A

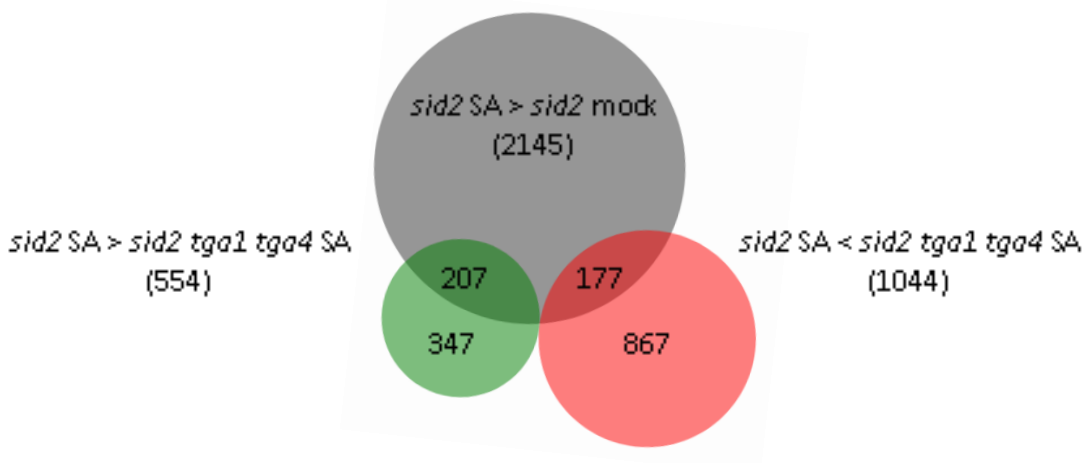

B

GO Biological Processes

response to external stimulus defense response response to biotic stimulus response to other organism response to external biotic stimulus defense response to other organism response to bacterium defense response to bacterium

Percentage of genes 0

\section{D}

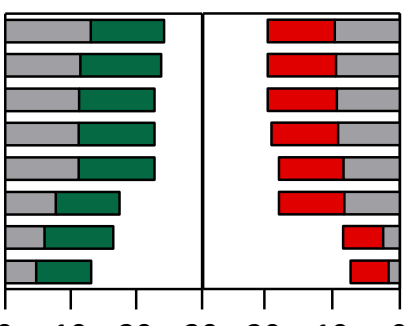

GO Biological Processes

regulation of nitrogen metabolic process regulation of biosynthetic process regulation of cellular biosynthetic process regulation of gene expression regulation of RNA metabolic process regulation of RNA biosynthetic process regulation of hormone levels hormone metabolic process

\section{\% SA inducible sid $2 \square \% 207$ genes $\square$ \% SA inducible sid $2 \square \% 177$ genes}

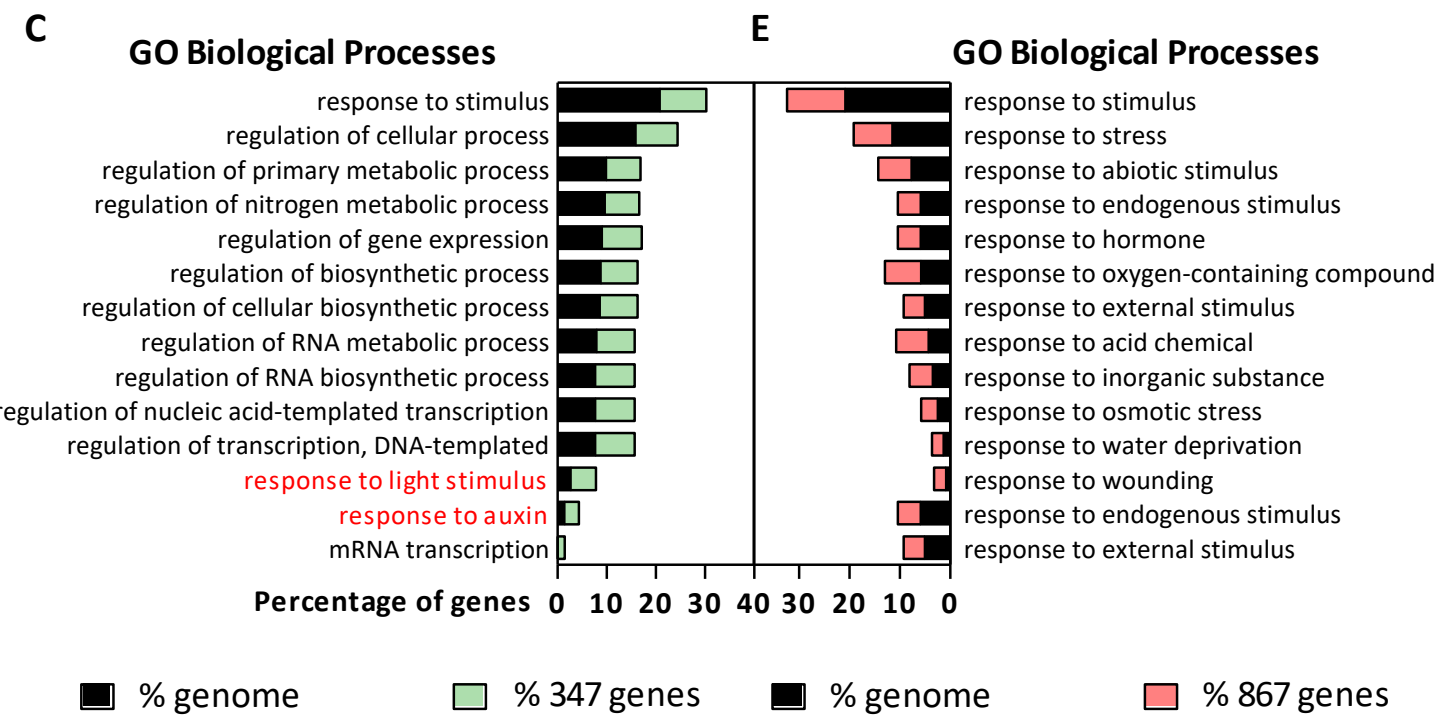

Figure 4 Analysis of genes which were less and more expressed in sid 2 tga 1 tga 4 mutant eight hours after SA treatment comparing to SA-inducible genes in sid2 background

A Venn diagram was used to analyze the relation of SA-inducible genes in sid2 (gray circle) and genes which were more (red circle) and less (green circle) expressed in sid2 tga1 tga4 mutant after SA treatment.

B The group of 207 genes, which were SA-inducible in sid2 and less expressed in the sid 2 tga 1 tga 4 mutant background was analyzed for the $\mathrm{GO}$ (Gene Ontology) enrichment in biological processes (dark green bars). The group of 2145 SA-inducible genes of sid 2 was used as a background set (grey bars). 
C The group of 347 genes, which were not SA-inducible in sid 2 but were less expressed in the sid 2 tga 1 tga 4 mutant background was analyzed for the GO (Gene Ontology) enrichment in biological processes (light green bars). The Arabidopsis genome was used as a background set (black bars). Indicated in red are GO terms which were previously found to be downregulated in tga1 tga4 mutant (Li et al, 2019).

D The group of 177 genes, which were SA-inducible in sid 2 and more expressed in the sid2 tga1 tga4 mutant background was analyzed for the GO (Gene Ontology) enrichment in biological processes (red bars). The group of 2145 SA-inducible genes of sid2 was used as a background set (grey bars).

E The group of 867 genes, which were not SA-inducible in sid2 but were more expressed in the sid2 tga1 tga 4 mutant background was analyzed for the GO (Gene Ontology) enrichment in biological processes (light red bars). The Arabidopsis genome was used as a background set (black bars).

Differentially expressed genes were determined as FC (fold change (log2 FC $\geq 1$ or $F C \leq-1), p<0.05$ ). Statistical analysis was performed using RobiNA software. Gene Ontology analysis platform was used for the GO enrichment analysis (http://geneontology.org/). The software uses Arabidopsis genome reference list consisting of 27581 genes. Statistical analysis was performed using Fisher test and False discovery rate $(F D R)<10^{-14}(B), F D R<10^{-3}(C), F D R<10^{-4}(\mathrm{D})$ and $F D R<10^{-6}(E)$. Top 8 most significant hit are shown for $\mathbf{B}$ and $\mathbf{D}$ and top 14 for $\mathbf{C}$ and $\mathbf{E}$.

\subsubsection{Induction of SARD1 is TGA1/TGA4-independent after SA treatment}

SARD1 is described as a direct target of TGA1/TGA4 after the pathogen infection (Sun et al. 2017). This transcription factor is not only important for activation of SA biosynthesis genes but also for induction of genes downstream of SA. However, it is not known how SARD1 is regulated after the SA treatment.

To address this question, we analyzed levels of SARD1 in mock- and SA-treated samples used for RNA sequencing. Transcript levels of SARD1 in sid2 tga1 tga4 mutant were lower than in sid2 mutant after the mock treatment but not after SA treatment, showing that the induction of SARD1 under described conditions is independent of TGA1/TGA4 (Figure 5A). Because SARD1 transcript is less abundant in sid2 tga1 tga 4 mutant after the mock treatment, we decided to test promoters of all the genes which were less expressed in the triple mutant under these conditions for enrichment of SARD1-binding motif. After mock treatment, there were 926 genes that were less expressed in sid2 tga1 tga4 than in sid2 (fold change (log2 FC $\leq-1), p<0.05)$. Promoters of identified genes were tested for enrichment of SARD1-binding site at 1000 base pair promoter region upstream of the Transcription Start Site (TSS) with Motif Mapper. The enrichment is calculated as compared to a background set, this being the promoter regions of either the whole Arabidopsis genome or a specific group of genes. The number of analyzed promoters from the background set is determined by the number of 
genes in the list of interest. For example, if there are 926 genes in the list of interest, the program will take 926 random promoters from the background set and analyze it. This process is repeat one thousand times. The resulting table consists of number of promoters in the compared lists, with the statistical significance of the difference.

In the first analysis, we compared the promoters of 926 genes that were less expressed in sid2 tga1 tga4 mutant after mock treatment with the promoters of the whole Arabidopsis genome. Analysis did not show significant enrichment of SARD1-binding site at the promoter regions of 926 genes (Figure 5B). To further exclude that transcriptional changes after SA treatment were affected by the low levels of SARD1 in the mock situation, we also tested promoters of 207 SA-inducible genes which were less expressed in sid 2 tga1 tga4 after SA treatment. As a background, we used promoters of 2145 SA-inducible genes in sid2. There was no significant enrichment of SARD1-binding site at the promoter regions of 207 genes (Figure 5B).
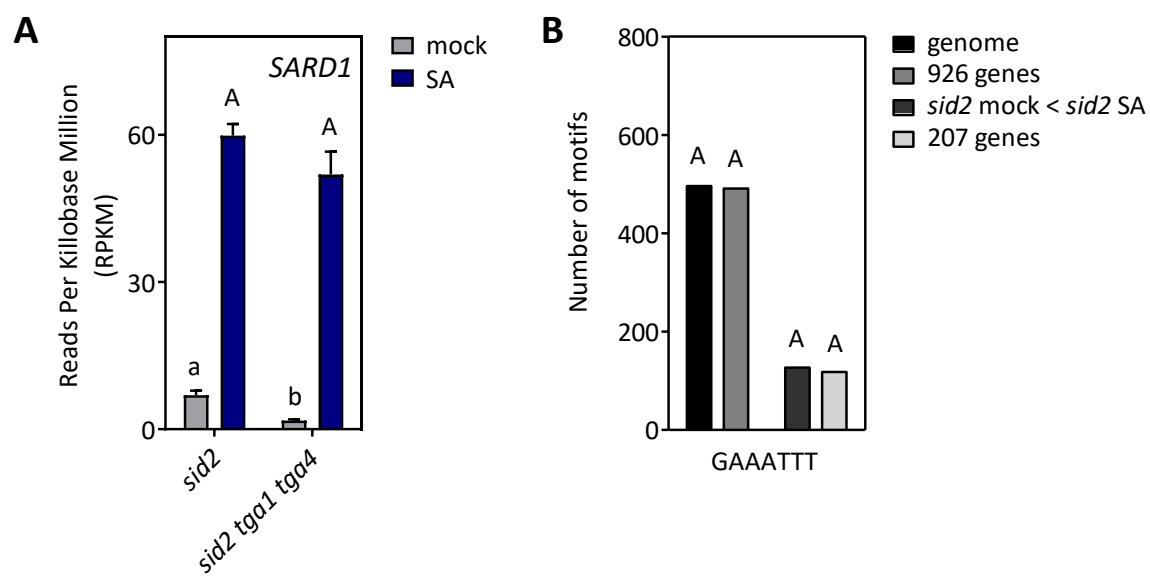

Figure 5 Transcriptional regulation of SARD1 is TGA1/TGA4-independent after SA treatment.

A Induction of SARD 1 after SA treatment in sid2 and sid 2 tga 1 tga 4 plants. Plants were sprayed with either mock or $1 \mathrm{mM} \mathrm{SA}$ at $1 \mathrm{~h}$ after the subjective dawn and further incubated for $8 \mathrm{~h}$. Transcriptome analysis was performed using Illumina sequencing. Bars represent the average of RPKM \pm SEM of four plants of each genotype.

B Promoter enrichment analysis of 926 and 207 genes that were less expressed after mock treatment (dark grey) and SA treatment (light grey) in sid2 tga1 tga 4 comparing to sid2, respectively. Enrichment of SARD1-binding sites at the 1000 kilobase promoter region was calculated using Motif Mapper software. The program compares the number of motifs in the promoter region of genes of interest with the number of motifs in the promoter region of genes taken a thousand times randomly from the background set.

Statistical analysis was performed using (A) RobiNA software. Lowercase letters indicate significant differences $(P<0.05)$ between mock-treated samples; uppercase letters indicate significant 


\section{Results}

differences $(P<0.05)$ between SA-treated samples. RPKM- Reads per Kilobase Million, mock-water, SA-salicylic acid.

\subsubsection{The promoter regions of SA-induced genes are enriched in TGA-binding sites}

The promoters of the identified 207 genes, which were SA-inducible in sid 2 and less expressed after SA treatment in sid2 tga1 tga4 mutant, were tested for enrichment of TGA-binding sites at 1000 base pair promoter region upstream of TSS using Motif Mapper software (Figure 6A). For the analysis, we chose known TGA-biding element, the extended C-box element (TGACG), the variations of the same (TGACGT(C/T)A, TGACGTAA) and the A-box element (TACGTA) (Izawa et al. 1993; Wang et al. 2019) (Table 19). The enrichment of TGA-binding sites was found when the whole Arabidopsis genome was used as a background set (Figure 6B). However, when the promoters of 2145 SA-induced genes were used as a background set, the enrichment was not observed. Thus, there was an enrichment of TGA-binding sites at the promoter regions of the SA-inducible genes in general, but no further enrichment was observed at the promoter regions of 207 genes of interest (Figure 6B and Table 19).

Table 19 Motif Mapper promoter enrichment analysis of 207 TGA1/TGA4-dependent genes

\begin{tabular}{l||r|r|l|r|l}
\multicolumn{1}{l||}{ Dataset } & \multicolumn{2}{l|}{ Background set $($ sid2 mock < sid2 SA) } & \multicolumn{2}{l}{ Background set (Genome) } \\
\hline \hline Motif & Number of motifs & Number of motifs & p value & Number of motifs & $p$ value \\
\hline \hline TGACGTCA & 6 & 4,414 & 0,227 & 2,659 & 0,019 \\
\hline TGACGT(C/T)A & 18 & 14,106 & 0,203 & 9,510 & 0,017 \\
\hline TGACGTAA & 10 & 8,147 & 0,305 & 4,231 & 0,003 \\
\hline TACGTA & 48 & 43,379 & 0,233 & 36,360 & 0,042
\end{tabular}


A

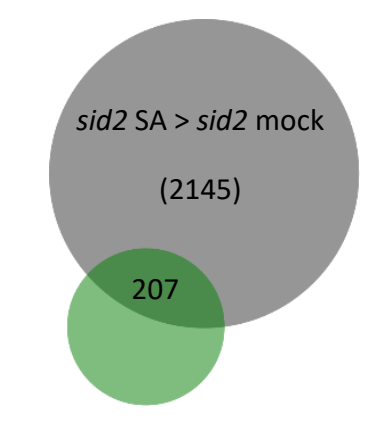

sid2 SA > sid2 tga1 tga4 SA

(554)
B

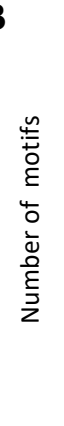

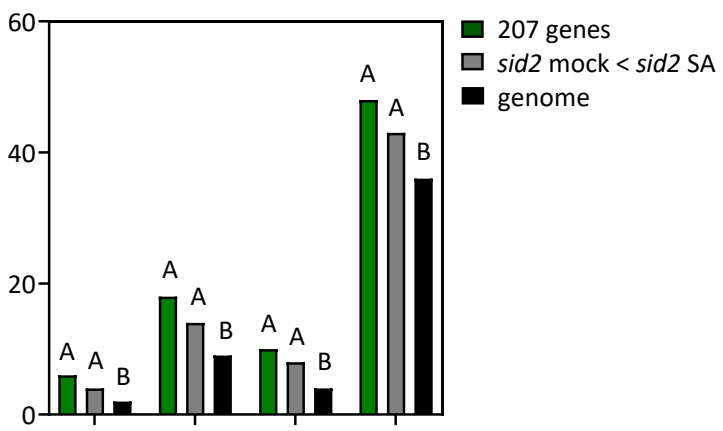

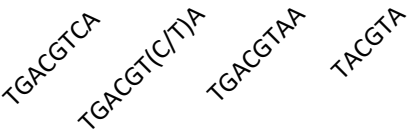

Figure 6 Promoters of 207 SA-inducible and TGA1/TGA4-dependent genes were enriched for TGAbinding sites.

A Venn diagram of 2145 SA-inducible genes in sid 2 and 554 genes which were less expressed after SA treatment in sid2 tga1 tga4 mutant than sid2. The overlapping region of 207 genes contains the TGA1/TGA4-dependent genes of interest.

B Promoter regions of 207 genes that were less expressed after SA treatment in sid 2 tga 1 tga 4 comparing to sid 2 were analyzed for the enrichment of TGA-binding sites at the 1000 kilobase promoter region. The extended C-box element (TGACG), which is a known TGA binding sequence, and the variations were analyzed for the enrichment. We also included A-box element, recently reported target of TGA1 (Wang et al. 2019).

Statistical analysis was performed using (A) RobiNA software with fold change (log2 FC $>1)$ or ( $\log 2 \mathrm{FC}$ $\leq-1)$ and $P<0.05)$ and (B) Motif Mapper software. Samples with a significant difference at $P<0.05$ are indicated with different letters. mock-water, SA-salicylic acid.

Although there was no significant enrichment in TGA-binding sites at the regions of the 207 TGA1/TGA4-dependent genes, we have identified several potential direct target genes (Error! R eference source not found.A). Due to a very strong dependency on TGA1/TGA4, we decided to use DLO1 (DOWNY MILDEW RESISTANCE 6-LIKE OXYGENASE 1) from this group as a marker gene for further analysis (Error! Reference source not found.B). DLO1 encodes for salicylic acid 3 -hydroxylase involved in SA catabolism (Zhang K. et al, 2013).

Additionally, we also chose two typical SA response marker genes which were less expressed in the absence of TGA1/TGA4, BGL2 (B-1-3-GLUCANASE 2) and PR1 (PATHOGENESIS RELATED 1) (Error! Reference source not found.B). Both genes also contain TGA binding sites at the promoter region (Error! Reference source not found.A). 
A

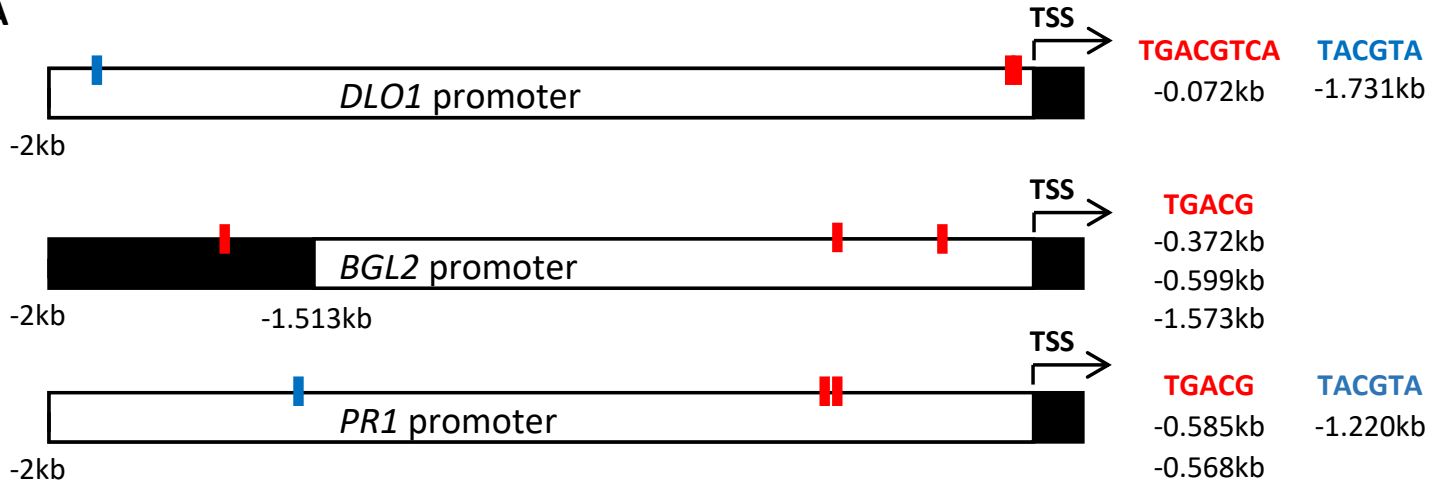

B
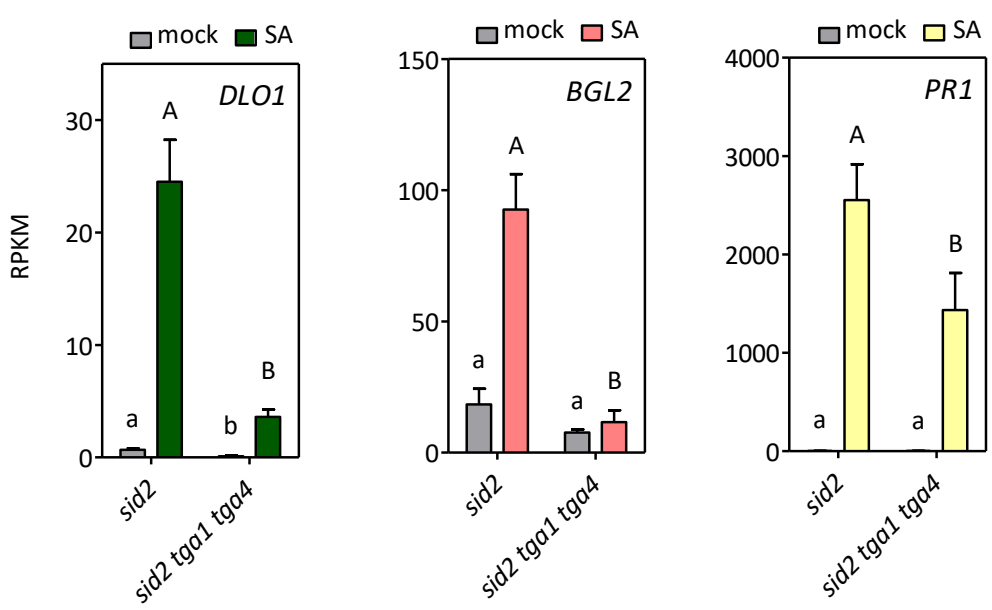

Figure 7 Expression of $D L O 1, B G L 2$ and $P R 1$ is TGA1/TGA4-dependent after SA treatment.

A Two thousand base pairs region upstream of Transcription Start Site (TSS) of DLO1, BGL2 and PR1 gene. The C-box element (TGACG) is shown in red and the A-box (TACGTA) in blue. Position upstream of TSS of the corresponding binding motif is indicated on the right side.

B Induction of DLO1 and BGL2 after SA treatment in sid 2 and sid 2 tga 1 tga 4 plants. Plants were sprayed either with mock or $1 \mathrm{mM} \mathrm{SA}$ at $1 \mathrm{~h}$ after the subjective dawn and further incubated for $8 \mathrm{~h}$. Transcriptome analysis was performed using Illumina sequencing. Bars represent the average of RPKM \pm SEM of four plants of each genotype.

Statistical analysis was performed using RobiNA software. Lowercase letters indicate significant differences $(P<0.05)$ between mock-treated samples; uppercase letters indicate significant differences $(\mathrm{P}<0.05)$ between SA-treated samples. RPKM-Reads per Kilobase Million, mock-water, SAsalicylic acid. 


\subsection{The group of TGA1/TGA4-dependent genes belongs to the classical}

\section{SA signaling pathway}

\subsubsection{TGA1/TGA4-dependent regulation of SA-inducible genes can be observed also in the} wild-type background

We were interested to test if the observations we detected in sid2 background were transferable to wild-type background (Columbia, Col-0). For this, we used Col-O wild-type plants and tga1 tga4 mutant together with sid 2 and sid 2 tga 1 tga 4 mutants and performed the SA-spraying experiment. Expression levels of marker genes were detected using qRT-PCR. The tga1 tga4 mutant had lower levels of DLO1 and PR1 then the wild-type, displaying the same trend as seen in sid2 background (Figure 8). In this particular experiment, BLG2 was not induced by SA treatment in wild-type plants but the trend of the overall lower expression in the absence of TGA1/TGA4 was still observed (Figure 8).
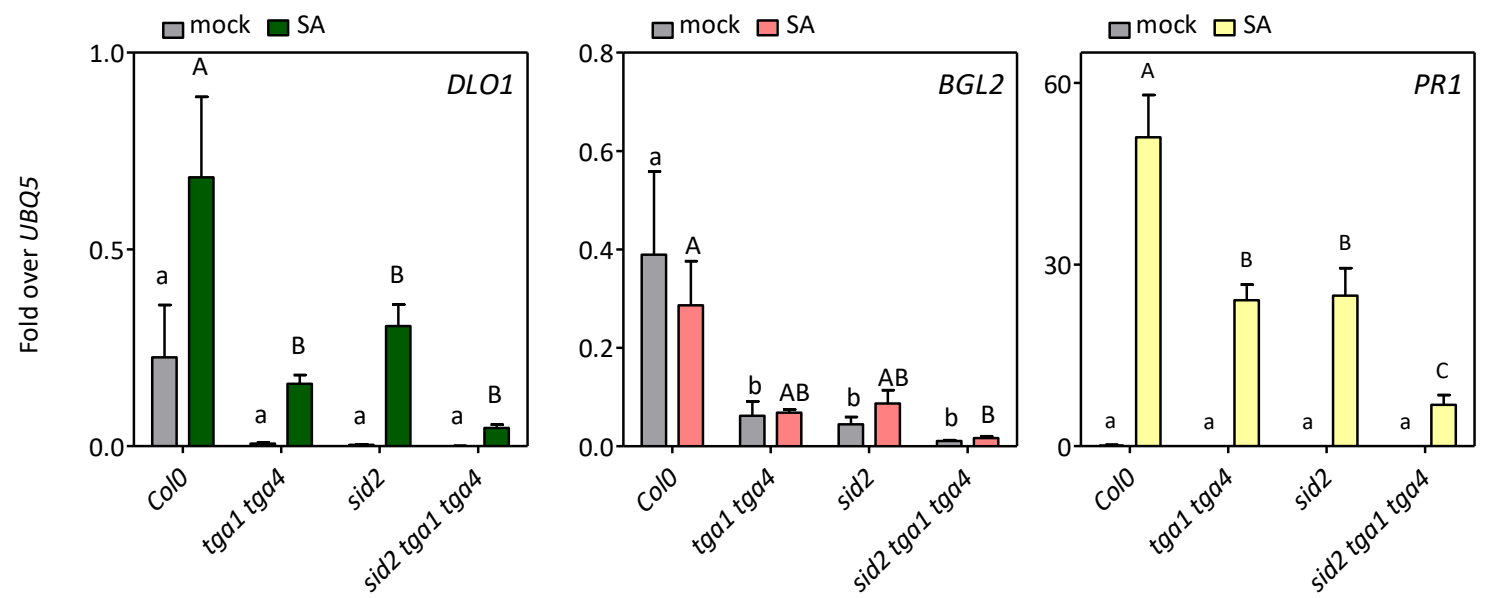

Figure 8 Induction of $D L O 1, B G L 2$ and $P R 1$ after SA treatment is TGA1/TGA4-depedent in wild-type and sid2 background.

qRT-PCR analysis of DLO1, BGL2 and PR1 transcript levels after SA treatment. Four-week-old plants were sprayed either with mock or $1 \mathrm{mM} \mathrm{SA}$ at $1 \mathrm{~h}$ after the subjective dawn and further incubated for $8 \mathrm{~h}$. Transcript levels were normalized to transcript level of UBQ5 (UBIQUITIN 5). Bars represent the average \pm SEM of four to six plants of each genotype. Experiment was performed once.

Statistical analysis was performed using two-way ANOVA followed by Bonferroni's post-hoc test. Lowercase letters indicate significant differences $(P<0.05)$ between mock-treated samples; uppercase letters indicate significant differences $(P<0.05)$ between SA-treated samples. mock-water, SA-salicylic acid. 


\subsubsection{Induction of TGA1/TGA4-regulated genes is TGA2/TGA5/TGA6- and NPR1-dependent} In order to verity that the genes which were regulated in TGA1/TGA4-depedent way belong to classical SA signaling pathway, we analyzed the mutants of genes encoding for the SA signaling components. Wild-type and mutant plants were treated with $\mathrm{SA}$, as previously described. Expression levels of DLO1, BGL2 and PR1 were determined by qRT-PCR. SAinduction which was observed in wild-type background was reduced not only in tga1 tga4 mutant but also in tga 2 tga 5 tga 6 and npr1 mutants (Figure 9). This same trend was observed in sid2 mutant background (Supplementary Figure 4).
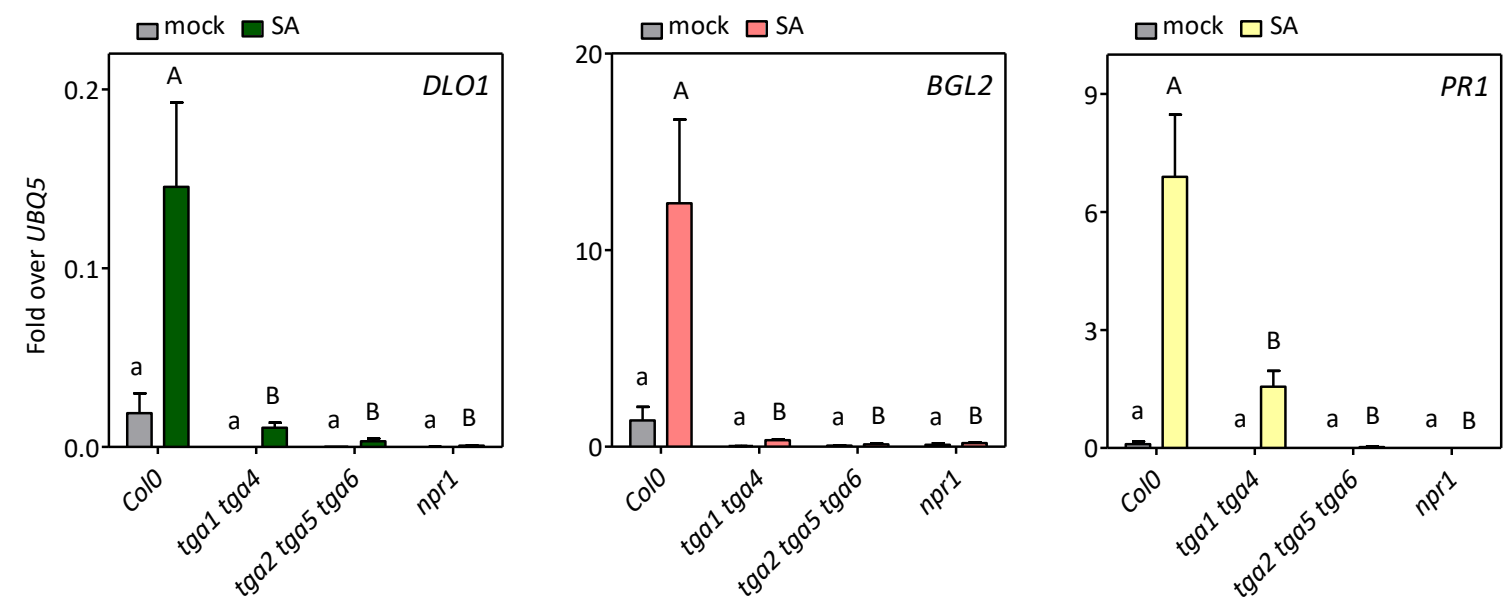

Figure 9 The group of TGA1/TGA4-dependent genes belongs to the classical SA pathway consisting of TGA2/TGA5/TGA6 and NPR1.

qRT-PCR analysis of DLO1, BGL2 and PR1 transcript levels after SA treatment of wild-type and tga1 tga4, tga 1 tga 5 tga 6 and npr 1 plants. Four-week-old plants were sprayed either with mock or $1 \mathrm{mM}$ $\mathrm{SA}$ at $1 \mathrm{~h}$ after the subjective dawn and further incubated for $8 \mathrm{~h}$. Transcript levels were normalized to transcript level of UBQ5. Bars represent the average \pm SEM of five to six plants of each genotype. Experiment was repeated once with similar results. All data shown here is from the same experiment.

Statistical analysis was performed using two-way ANOVA followed by Bonferroni's post-hoc test. Lowercase letters indicate significant differences $(P<0.05)$ between mock-treated samples; uppercase letters indicate significant differences $(P<0.05)$ between SA-treated samples. mock-water, SA-salicylic acid. 


\subsubsection{DLO1 is a potential direct target of TGA1/TGA4, TGA2/TGA5/TGA6 and SARD1}

Interestingly, DLO1 promoter contains only one TGA binding site, which can be either occupied by TGA1, TGA2 or heterodimer of TGA1 and TGA2. In order to elucidate how DLO1 is regulated by both clade I and II TGA transcription factors, which do not form heterodimers often, we went back to the promoter sequence and looked for other potential regulators (Niggerwer et al. 2000). We found a binding site for SARD1, another SA-responsive transcription factor (Figure 10A). We previously showed that regulation of SARD1 is not TGA1/TGA4-dependent upon SA treatment (Figure 10A). This would mean that TGA1/TGA4 were not responsible for indirect regulation of DLO1 through SARD1. Thus, we hypothesized that TGA2/TGA5/TGA6 act through SARD1. To test this, we analyzed levels of SARD1 in tga2 tga5 tga6 mutant plants after SA treatment. Indeed, SARD1 transcript levels were depleted in the absence of TGA2/TGA5/TGA6 and NPR1 while this was not the case in the absence of TGA1/TGA4 (Figure 10B). The same trend was also observed when sid2 mutant background was used (Supplementary Figure 5). This data implied that DLO1 expression would also be affected by the absence of SARD1. To address this question, we used double mutant of SARD1 and its closest homologue $C B P 60 \mathrm{~g}$, sard1 $\mathrm{cbp} 60 \mathrm{~g}$ and performed SA spraying experiment. The transcript levels of DLO1 were decreased in the mutant background compared to wild-type plants (Figure 10C). Moreover, expression levels $B G L 2$ and $P R 1$ are also significantly lower in sard1 cbp60g mutant compared to the wild-type plants (Figure 10C).

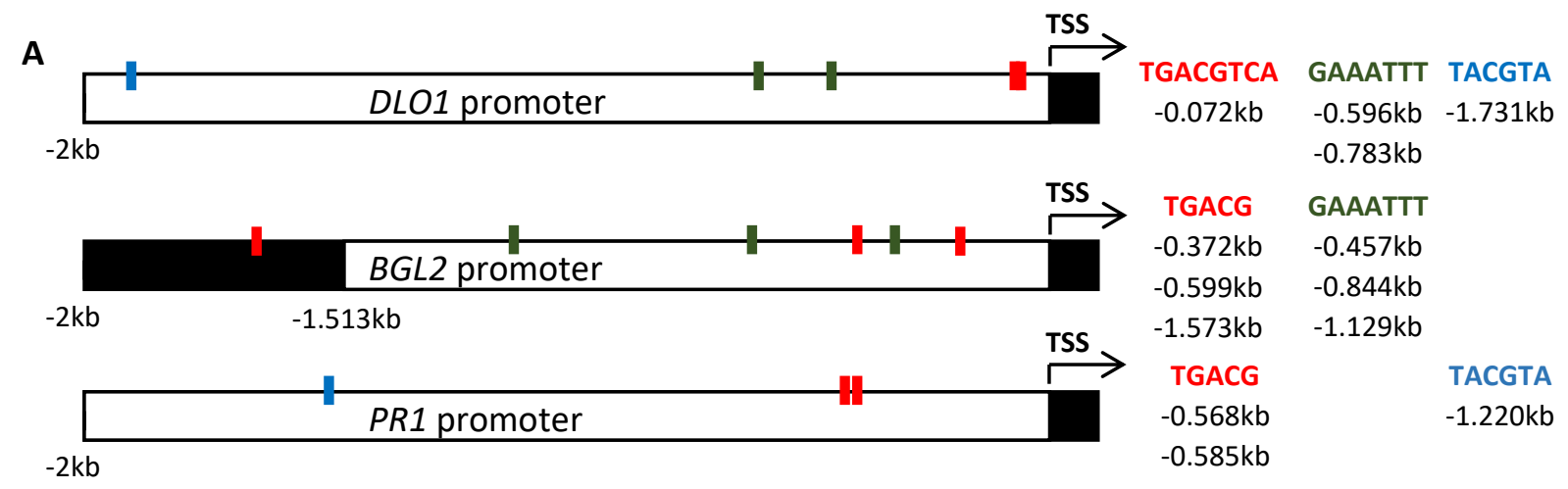


B

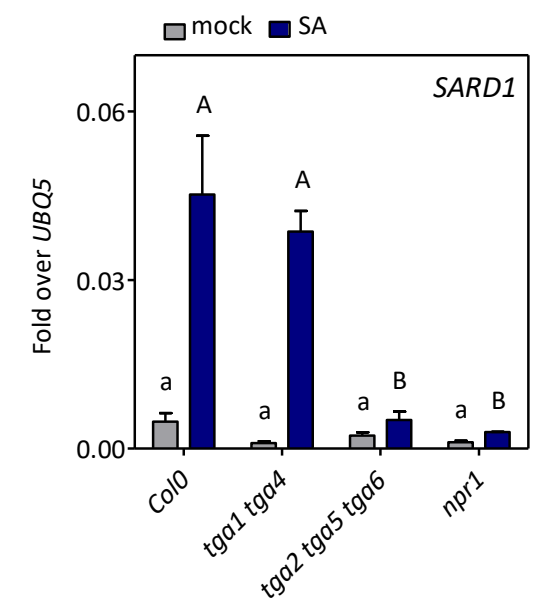

C

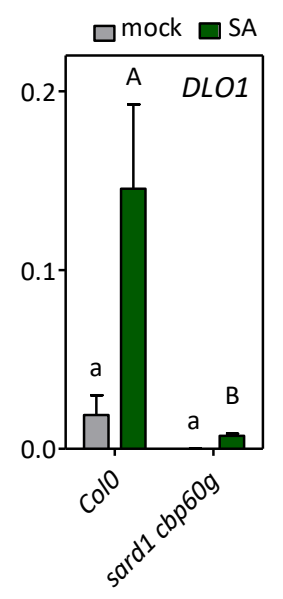

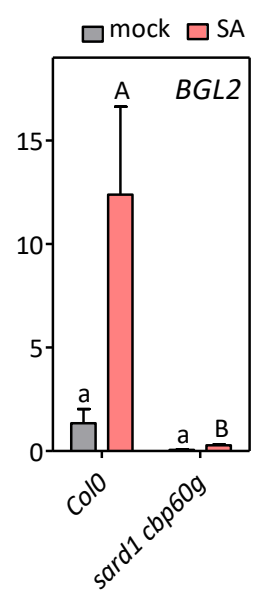

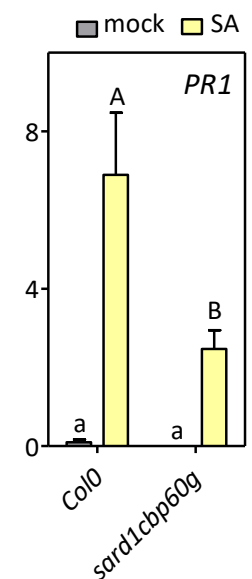

Figure 10 NPR1 and TGA2/TGA5/TGA6 indirectly activate DLO1 transcription through SARD1.

A Two thousand base pairs region upstream of Transcription Start Site (TSS) of DLO1, BGL2 and PR1 gene. The C-box element (TGACG) is shown in red and the A-box element (TACGTA) in blue. SARD1binding motif (GAAATTT) is shown in green. Position upstream of TSS of the corresponding binding motif is indicated on the right side.

B qRT-PCR analysis of SARD1 transcript levels after SA treatment of wild-type and tga1 tga4, tga1 tga5 tga 6 and $n p r 1$ plants. Four-week-old plants were sprayed either with mock or $1 \mathrm{mM} \mathrm{SA}$ at $1 \mathrm{~h}$ after the subjective dawn and further incubated for $8 \mathrm{~h}$. Transcript levels were normalized to transcript level of $U B Q 5$. Bars represent the average \pm SEM of five to six plants of each genotype. Experiment was repeated once with similar results. All data shown here is from the same experiment.

C qRT-PCR analysis of DLO1, BGL2 and PR1 transcript levels after SA treatment of wild-type and sard1 cbp60g plants. Four-week-old plants were sprayed either with mock or $1 \mathrm{mM} \mathrm{SA}$ at $1 \mathrm{~h}$ after the subjective dawn and further incubated for $8 \mathrm{~h}$. Transcript levels were normalized to transcript level of UBQ5. Bars represent the average \pm SEM of five to six plants of each genotype. Experiment was repeated once with similar results. All data shown here are from the same experiment (B and $\mathbf{C}$ ).

Statistical analysis was performed using two-way ANOVA followed by Bonferroni's post-hoc test. Lowercase letters indicate significant differences $(P<0.05)$ between mock-treated samples; uppercase letters indicate significant differences $(P<0.05)$ between SA-treated samples. mock-water, SA-salicylic acid. 


\subsection{The redox state of the four critical cysteine residues of TGA1 is not important for its transcriptional activity}

\subsubsection{The redox state of the four critical cysteine residues is not important for induction of TGA1/TGA4-dependent genes downstream of SA}

Clade I TGA transcription factors differ from the other members of the TGA family due to the presence of the four critical cysteine residues (Cys 172, Cys 260, Cys 266, Cys 287). The two inner cysteines (Cys 260 and Cys 266) form a disulfide bridge which can be reduced upon salicylic treatment in vivo (Després et al. 2003) and the two outermost (Cys172 and Cys 287) can be nitrosylated and glutathionylated in vitro (Lindermayr et al. 2010). The reduction of a disulfide bridge by increased levels of SA enables interaction of TGA1/TGA4 with NPR1 and was proposed by Després and colleagues as a regulatory mode of clade I TGA transcription factors. This model implies TGA1 as a SA-switchable protein. However, the in vivo evidence was missing. Therefore, we wanted to address if the redox state of the critical cysteine residues plays a role in the function of TGA1 downstream of SA. To do so, the tga1 tga4 mutant was either complemented with HA (Human influenza hemagglutinin) tagged genomic sequence of TGA1 (from now on TGA1g) or genomic sequence with mutations in four cysteine residues to mimic reduced form of the protein (from now on TGA1gr) under the native promoter. The tga1 tga4 double mutant was also complemented with the empty vector (HA tag-3'UTR of TGA1 from now on EV) to serve as a transformation control. All the transgenic lines were prepared by Katrin Treffon. For further analysis, we chose two pairs of individual lines which displayed similar protein levels (Figure 11A).

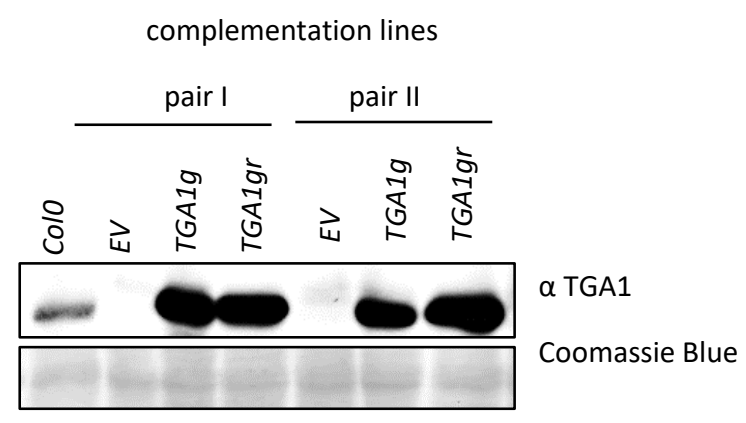

Figure 11 TGA1 protein could be detected in wild-type plants and the complementation lines. 
Western blot analysis of the root protein extracts of wild-type plants and plants expressing empty vector (EV), TGA1 genomic clone (TGA1g) or TGA1 genomic clone carrying mutations in four critical cysteine residues (TGA1gr) under native promoter. TGA1 protein was detected using TGA1 antibody.

Finally, we could use the identified TGA1/TGA4- and NPR1-dependent genes to analyze the response of the different complementation lines to SA treatment. The SA spraying experiment was performed as previously described. DLO1, BLG2 and $P R 1$ expression was strongly induced by SA treatment. Gene expression in EV control, representing the tga 1 tga 4 mutant, was lower than in the wild-type plants (Figure 12). There was no significant difference in the gene expression between TGA1g and TGA1gr complementation (Figure 12 and Supplementary Figure 3).
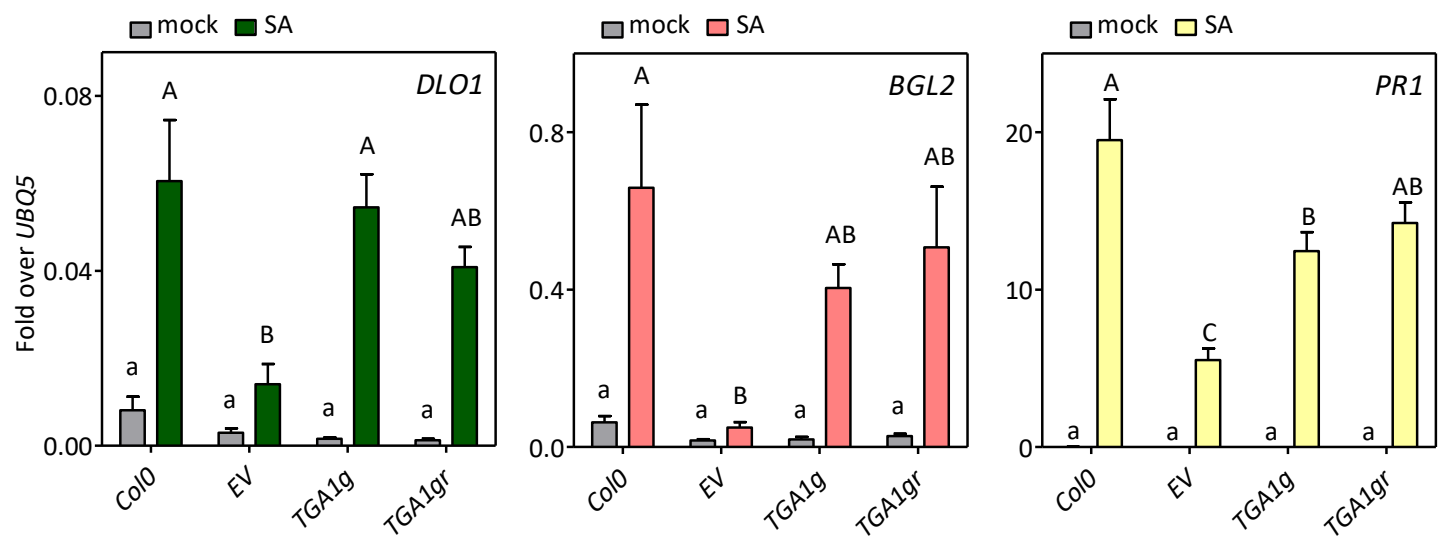

Figure 12 Complementation of tga1 tga4 mutant is not influenced by the redox state of the four critical cysteine residues.

qRT-PCR analysis of DLO1, BGL2 and PR1 transcript levels after SA treatment of wild-type and tga1 tga4 plants complimented either with empty vector (EV), TGA1 genomic clone (TGA1g) or TGA1 genomic clone carrying mutations in the four critical cysteine residues (TGA1gr) under native promoter. Four-week-old plants were sprayed either with mock or $1 \mathrm{mM} \mathrm{SA}$ at $1 \mathrm{~h}$ after the subjective dawn and further incubated for $8 \mathrm{~h}$. Transcript levels were normalized to transcript level of UBQ5. Bars represent the average \pm SEM of four to six plants of each genotype. Experiment was repeated once with similar results. All data shown here are from the same experiment.

Statistical analysis was performed using two-way ANOVA followed by Bonferroni's post-hoc test. Lowercase letters indicate significant differences $(P<0.05)$ between mock-treated samples; uppercase letters indicate significant differences $(P<0.05)$ between SA-treated samples. EV-empty vector, TGA1TGA1 genomic clone, TGA1gr-TGA1 genomic clone with four cysteines mutated, mock-water, SAsalicylic acid. 


\subsection{Induction of TGA1-regulated genes after pathogen infection is dependent on clade I and II TGA transcription factors and NPR1}

\subsubsection{TGA1/TGA4 are important for induction of the target genes after infection with hemibiotrophic pathogen Pseudomonas syringae pv. maculicola}

The tga1 tga4 mutant shows increased susceptibility to hemibiotrophic pathogen, Pseudomonaas syringae pv. maculicola (Psm) due to the defects in SA and Pip production following the infection. This was due to the TGA1-dependent regulation of two transcription factors, SARD1 and CBP60g, which are activators of SA and Pip biosynthesis genes (Sun et al. 2018). Moreover, the tga1 tga4 mutant was more susceptible to the secondary infection making it deficient in SAR (Systemic Acquired Resistance). However, the genes which are regulated by TGA1/TGA4 in systemic tissue have not yet been described. Therefore, we decided to test if the genes found as TGA1/TGA4-dependent after SA treatment, such as DLO1 and $B G L 2$, were also inducible by the infection.

The next step was to see if DLO1 and BGL2 were still TGA1/TGA4-dependent under these conditions and if this induction is established through the same signaling cascade downstream of SA including NPR1, TGA2/TGA5/TGA6 and SARD1. In order to test this, we used loss of function mutants of the signaling components (sid2, npr1, tga1 tga4, tga 2 tga5 tga 6 and sard1 cbp60g) and performed SAR experiment. For this experiment, plants were either pretreated with Pseudomonas syringae (Psm) bacteria or magnesium chloride (mock) solution. The second Psm infection was performed two days after. All samples were collected eight hours after the second treatment. As a readout, qRT-PCR analysis was performed. In addition to $D L O 1$ and $B G L 2$, we analyzed expression levels of $S A R D 1$, which was reported as a direct target of TGA1 after Psm infection (Sun et al. 2018).

As opposed to what we have observed with SA-treated tga1 tga4 mutant plants, SARD1 was strongly dependent on TGA1/TGA4 after Psm infection. Likewise, the expression levels of DLO1 and BLG2 were reduced in the absence of TGA1/TGA4. All three analyzed genes were less expressed in the mutants of the SA signaling components (Figure 13). Moreover, the induction of DLO1 and BGL2 was stronger in the plants pretreated with Psm (dark blue) compared to the mock pretreated plants (light blue) (Figure 13). 

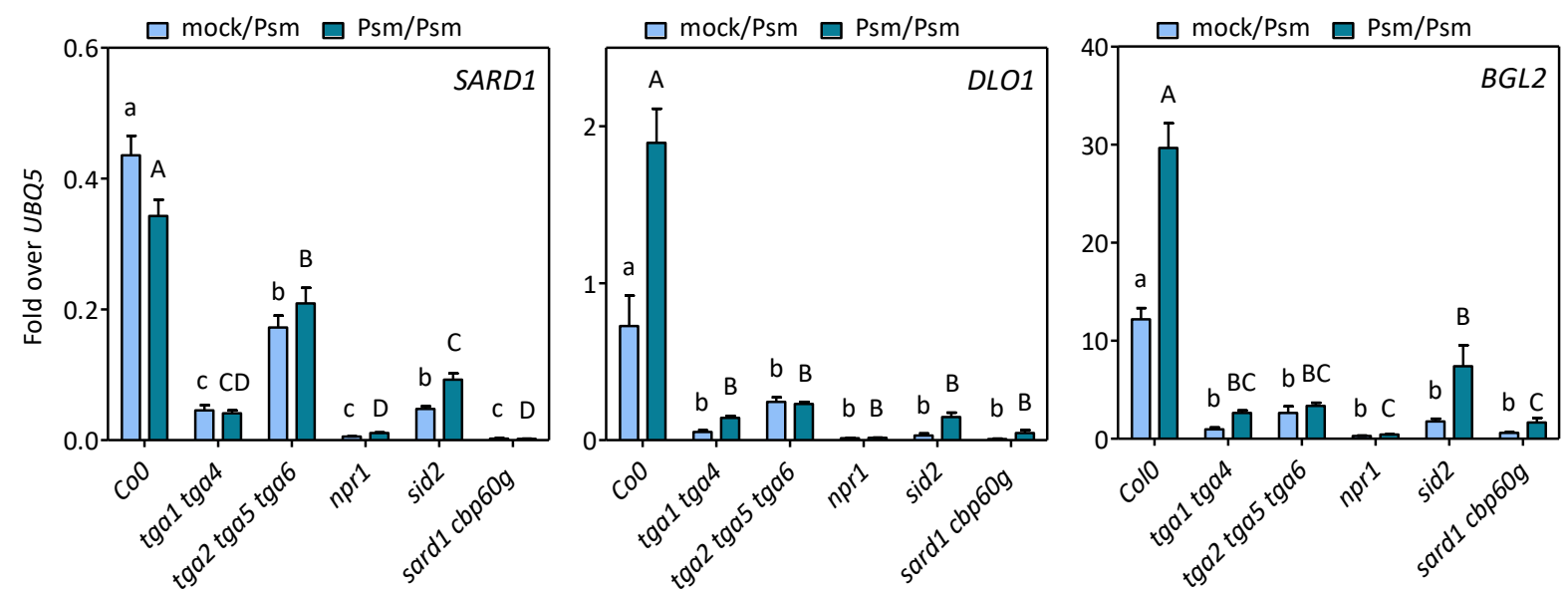

Figure 13 Expression of SARD1, DLO1 and BGL2 is induced after infection with Pseudomonas syringae and it requires TGA1/TGA4, TGA2/TGA5/TGA6, NPR1, SA and SARD1.

qRT-PCR analysis of SARD1, DLO1 and BGL2 transcript levels of wild-type, tga1 tga4, tga2 tga5 tga6, sid2, npr1 and sard1 cbp60g plants after Psm infection of plants that were either pretreated with $\mathrm{MgCl} 2$ (mock) or inoculated with Psm. Three older lower leaves of the five-week-old plants were mockor Psm-pretreated. After two days, three younger upper leaves were treated with Psm. These were collected $8 \mathrm{~h}$ after the second treatment and RNA was extracted. Transcript levels were normalized to transcript level of UBQ5. Bars represent the average \pm SEM of three to four plants of each treatment.

Statistical analysis was performed using two-way ANOVA followed by Bonferroni's post-hoc test. Lowercase letters indicate significant differences $(P<0.05)$ between mock-pretreated samples; uppercase letters indicate significant differences $(P<0.05)$ between Psm-infected samples. mockmagnesium chloride pretreated, Psm-Pseudomonas infected.

\subsubsection{The redox state of the four critical cysteine residues is not important for induction of TGA1/TGA4-dependent genes upon pathogen attack in SAR experiment}

Systemic Acquired Resistance leads to accumulation of SA which was postulated to regulate TGA1 activity (Després, 2003). Additionally, pathogen infection induces accumulation of nitric oxide (NO) which triggers transcription of defense genes and which was proposed as a regulator of TGA1 (Delledonne et al. 1998; Feechan et al. 2005; Lindermayr, 2010). Therefore, pathogen infection is a nice system to address the importance of TGA1-redox state for its activity.

We used the described complementation lines for an SAR experiment. The experiment was performed as described in the previous paragraph and we analyzed SARD1, DLO1 and BGL2 levels using qRT-PCR. There was a strong induction of all genes in systemic tissue when plants were pretreated with Psm as compared to the mock pretreatment, showing the priming effect 
(Figure 14-light blue bars versus dark blue bars). The expression of SARD1, DLO1 and BGL2 was drastically decreased in transgenic plants transformed with the empty vector (EV) control, representing the tga1 tga4 mutant (Figure 14). However, the difference in the gene expression between in the two types of complementation lines was not observed. This data confirmed the observation that the redox state of the four critical cysteine residues of TGA1 is not important for its activity.
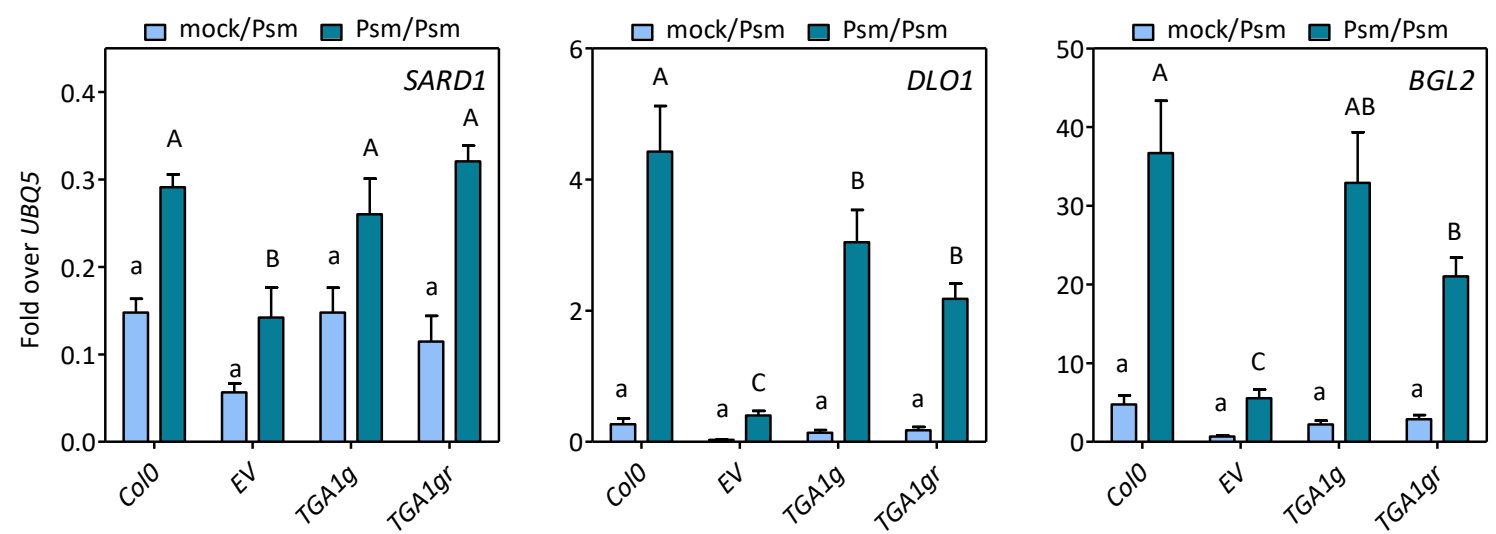

Figure 14 Complementation of $\operatorname{tga} 1 \operatorname{tga} 4$ mutant is not influenced by the redox state of the four critical cysteine residues after the infection with Pseudomonas syringae.

qRT-PCR analysis of SARD1, DLO1 and BGL2 transcript levels of wild-type and tga1 tga4 plants complimented either with empty vector (EV), TGA1 genomic clone (TGA1g) or TGA1 genomic clone carrying mutations in the four critical cysteine residues (TGA1gr) under native promoter. Plants were subjected to the SAR experiment. Three older lower leaves of the five-week-old plants were mock- or Psm-pretreated. After two days, three younger upper leaves were treated with Psm. These were collected $8 \mathrm{~h}$ after the second treatment and RNA was extracted. Transcript levels were normalized to transcript level of $U B Q 5$. Bars represent the average \pm SEM of five to six plants of each treatment.

Statistical analysis was performed using two-way ANOVA followed by Bonferroni's post-hoc test. Lowercase letters indicate significant differences $(P<0.05)$ between mock-pretreated samples; uppercase letters indicate significant differences $(P<0.05)$ between $P$ sm-infected samples. $E V$-empty vector, TGA1-TGA1 genomic clone, TGA1gr-TGA1 genomic clone with four cysteines mutated, mockmagnesium chloride pretreated, Psm-Pseudomonas infected. 


\subsection{TGA1 protein is predominantly expressed in roots and vascular tissue of Arabidopsis thaliana}

\subsubsection{TGA1 protein is abundant in roots of Arabidopsis thaliana}

During the time of this thesis, we have tried numerous times to perform Chromatin Immunoprecipitation (ChIP) experiment with the complementation lines. If successful, we could use this experiment to test if both versions of TGA1 (TGA1g and TGA1gr) would bind to the promoter of the identified potential target genes (DLO1). Unfortunately, we were not able to establish ChIP experiment. But we were not the first ones to encounter this problem. Sun and colleagues were equally unsuccessful with the ChIP from Arabidopsis leaves and they decided to use Arabidopsis mesophyll protoplast (Sun et al. 2018). The only successful TGA1 ChIP assay was performed with roots of hydroponically grown Arabidopsis plant (Alvarez et al. 2014).

Therefore, we analyzed the TGA1 protein levels and the TGA1 gene expression in the leaf and the roots extracts of Arabidopsis plant. For this, we used wild-type plants and the complementation lines. The specificity of the TGA1 antibody was confirmed using recombinant TGA1 and TGA4 protein from Escherichia coli (Supplementary Figure 6). Although we could not detect the protein in the leaf extract, the signal in the root extract was strong (Figure 15A). Likewise, the levels of TGA1 were higher in the root then the leaf extract (Figure 15B).

A

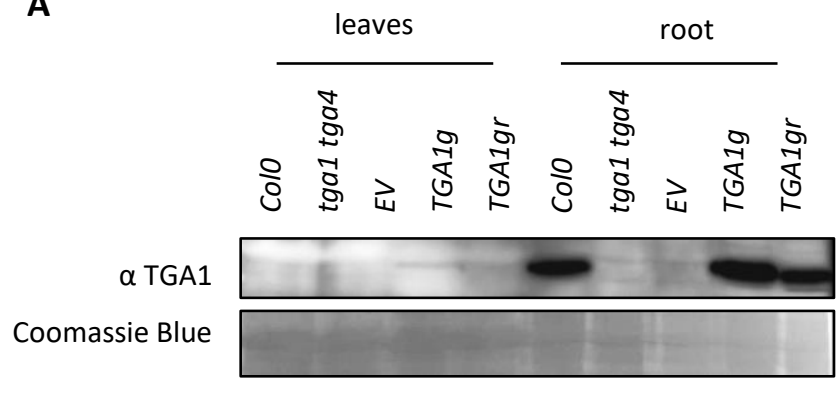

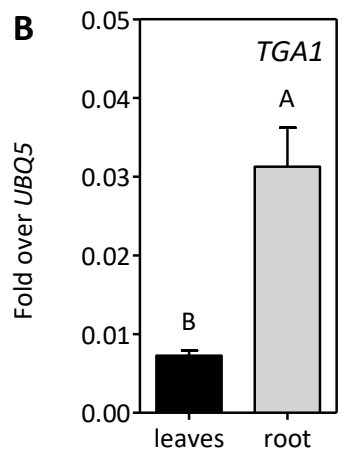

Figure 15 TGA1 is more abundant in the root than the leaves of Arabidopsis thaliana.

A Western blot analysis of protein extracts from leaves and roots of wild-type plants and plants expressing empty vector (EV), TGA1 genomic clone (TGA1g) or TGA1 genomic clone carrying mutations 
in the four critical cysteine residues (TGA1gr) under native promoter. TGA1 protein was detected using TGA1 antibody.

B qRT-PCR analysis of TGA1 transcript levels of mock treated leaves and untreated roots of wild-type plants. Leaves samples were collected from four-week-old plants sprayed with mock at $1 \mathrm{~h}$ after the subjective dawn and further incubated for $8 \mathrm{~h}$. Root samples were obtained from four-week-old soil grown untreated plants. Transcript levels were normalized to transcript level of UBQ5. Bars represent the average \pm SEM of six plants.

Statistical analysis was performed using two-tailed t-test. Samples with a significant difference at $P<$ 0.05 are indicated with different letters. EV-empty vector, TGA1-TGA1 genomic clone, TGA1gr-TGA1 genomic clone with four cysteines mutated.

As seen in Figure 15, TGA1 mRNA was detectable in the leaf extract but the protein was not. Therefore, we thought that perhaps some very abundant leaf protein, which had a similar size as TGA1, was covering TGA1 protein making it inaccessible to the antibody. In order to test this hypothesis, we performed a mixing experiment. In this experiment, the protein extract of the leaf tissue was mixed with the protein extract of the root tissue in different ratios (

Figure 16). Once again, TGA1 protein could be detected only when the root extract was added to the leaf extract (

Figure 16). We concluded that the overall amount of TGA1 protein in the leaf extract was below detectable.

\begin{tabular}{r|c|c|c|c|c|c|c|c} 
Col0 leaf $(\mu \mathrm{g})$ & 20 & 20 & 20 & 20 & 10 & $/$ & $/$ & $/$ \\
\hline Col0 root $(\mu \mathrm{g})$ & $/$ & 10 & 5 & 1 & 1 & $/$ & 5 & 10 \\
\hline tga1 tga4 leaf $(\mu \mathrm{g})$ & $/$ & $/$ & $/$ & $/$ & $/$ & 20 & 10 & $/$ \\
aTGA1 \\
\cline { 2 - 6 } \\
Coomassie Blue
\end{tabular}

Figure 16 TGA1 protein can be detected when leaf and root protein extracts are mixed.

Western blot analysis of protein extracts from leaves and roots of wild-type and tga1 tga4 mutant plants. Different ratios of leaf and root protein extract of wild-type plant were mixed together. Ratios are depicted in the table above the blot. TGA1 protein was detected using TGA1 antibody. Experiment was performed by Dyari Mohammed. 


\subsubsection{TGA1 promoter is expressed in vascular tissue and in roots of Arabidopsis thaliana} TGA1 promoter: GUS ( $\beta$-glucuronidase) protein fusion was used to address the TGA1 promoter expression. The lines were described and kindly provided by Shelley R. Hepworth (Carleton University, Ottawa, Canada) (Wang et al. 2019). The experiment is based on the activity of GUS protein to catalyze the substrate to the final product which can be visualized in the tissue where the gene promoter was active.

For this experiment, a three-week old plant including the root was used. A substrate of GUS, X-Gluc was added and incubated over night at $37^{\circ} \mathrm{C}$ in the dark. The product of the reaction can be easily visualized due to the blue color. As shown in Figure 17, strongest promoter activity was in the root, followed by the vascular tissue of older and younger leaves. As opposed to the published data where TGA1 promoter was detected only in root, vascular tissue and apices, we have detected it in the whole plant (Wang et al., 2019).

A
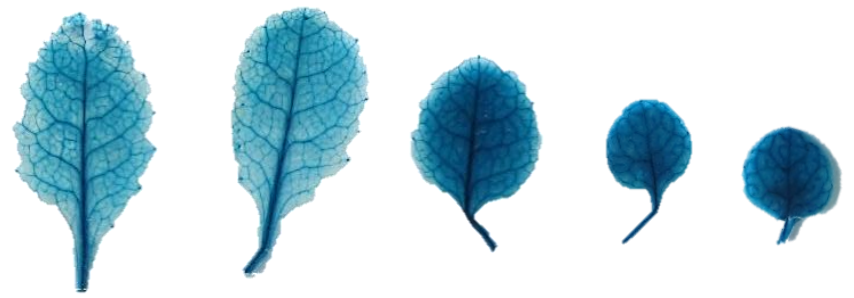

$1 \mathrm{~cm}$

B

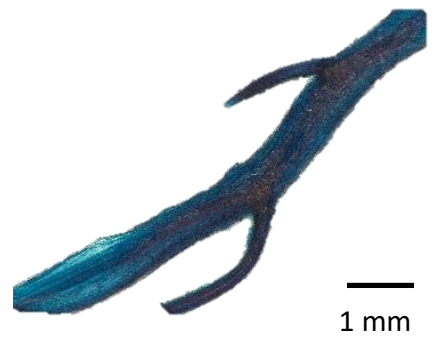

C

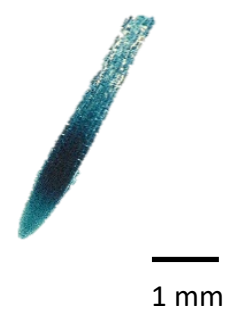

Figure 17 GUS protein under control of TGA1 promoter in shoot and root of Arabidopsis.

Three-week-old sol-grown TGA1promoter: GUS transgenic plant was collected, and the soil was washed from the root tissue. The whole plant was fixed with $0.3 \%$ formaldehyde and $0.3 \mathrm{M}$ mannitol solution. GUS staining solution with $2.5 \mathrm{mM}$ X-Gluc substate was vacuum infiltrated and plant was incubated over night at $37^{\circ} \mathrm{C}$ in the dark. Chlorophyll was washed with $100 \%$ ethanol and photos were taken.

A Protein accumulated in the vascular tissue and accumulation increased with leaf age.

B Protein was strongly expressed in root tissue.

C High expression was also noted in the root elongation zone of lateral roots. 


\section{Results}

Samples were visualized by digital microscope (VHX-500F KEYENCE) at 200 times amplification

\subsubsection{TGA1 does not accumulate in the leaves or the midrib after SA treatment, but does} after Pseudomonas syringae infection in the systemic tissue

After visualizing a very strong TGA1 promoter activity in the vascular tissue, we wanted to test protein abundance in this tissue. To do so, we divided the midrib from the rest of the leaf and extracted the protein. We also treated samples with either water or SA to see if there is any accumulation after the treatment. Again, only the protein extracted from the root was detected with TGA1 antibody (Figure 18A).

Because TGA1 was important for induction of SARD1, DLO1 and BGL2 after the Psm-infection, we were interested to see if we can detect the protein after the infection. For this analysis, we used the systemic tissue from the SAR leaves of the complementation lines. A band corresponding to the TGA1 protein band was detected (Figure 18B). We did not know if this observation was due to the induction of TGA1 gene or the stabilization of TGA1 protein. Thus, we analyzed TGA1 mRNA levels of the complementation lines from the SA spraying and SAR experiment using qRT-PCR. We have seen induction of TGA1 after SA (Figure 18C) as well after pathogen infection (Figure 18D). However, the protein was only detected after the SAR experiment (Figure 18B). The levels of TGA1 after SAR were approximately 20 times higher than after SA spraying.

A

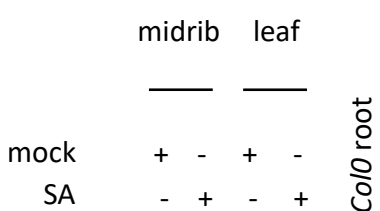

$\alpha$ TGA1

Coomassie Blue
B

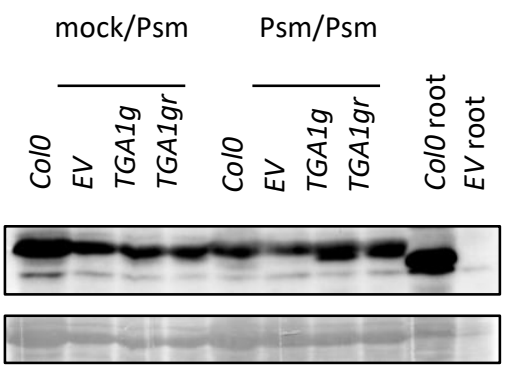



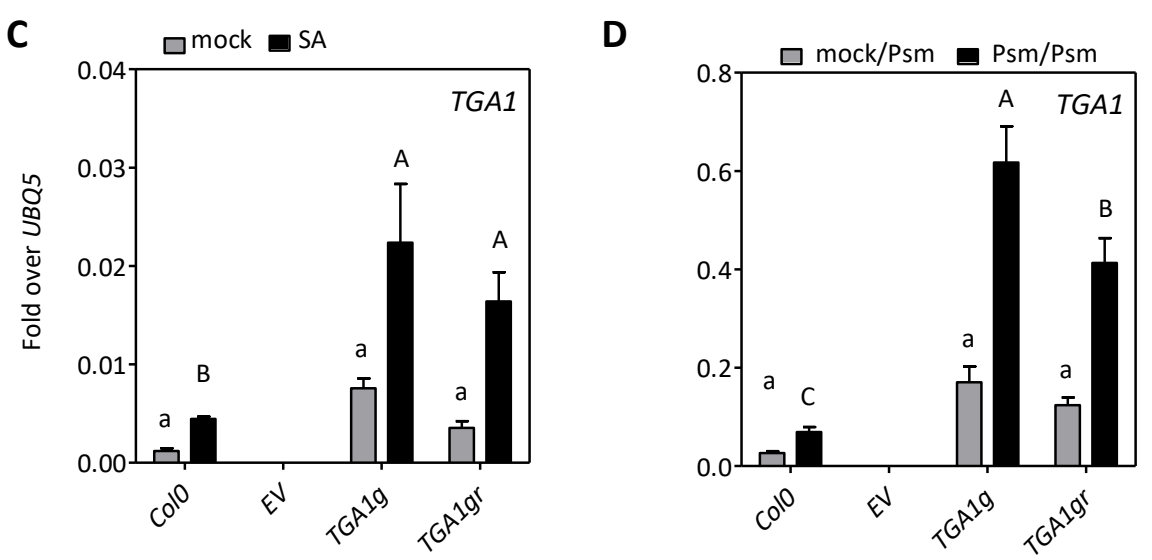

Figure 18 TGA1 transcription is induced after SA treatment and infection with Pseudomonas syringae

A Western blot analysis of protein extracts from leaves or midrib of wild-type plants. Four-week-old plants were sprayed either with mock or $1 \mathrm{mM}$ SA at $1 \mathrm{~h}$ after the subjective dawn and further incubated for $8 \mathrm{~h}$. Proteins were extracted from three leaves or four to six midribs of a single plant. Table above the Western blot indicates if the plant was treated with mock or SA. Root protein extract of wild-type plant was loaded as a positive control. TGA1 protein was detected by TGA1 antibody.

B Western blot analysis of protein extracts from leaves of wild-type plants and plants expressing empty vector (EV), TGA1 genomic clone (TGA1g) or TGA1 genomic clone carrying mutations in four critical cysteine residues (TGA1gr) under native promoter. Plants were subjected to the SAR experiment. Three older lower leaves of the five-week-old plants were mock- or Psm-pretreated. After two days, three younger upper leaves were treated with Psm. These were collected $8 \mathrm{~h}$ after the second treatment and proteins were extracted. Proteins were detected by TGA1 antibody.

C qRT-PCR analysis of TGA1 transcript levels after SA treatment of wild-type and tga1 tga 4 plants complimented either with empty vector (EV), TGA1 genomic clone (TGA1g) or TGA1 genomic clone carrying mutations in four critical cysteine residues (TGA1gr) under native promoter. Four-week-old plants were sprayed either with mock or $1 \mathrm{mM}$ SA at $1 \mathrm{~h}$ after the subjective dawn and further incubated for $8 \mathrm{~h}$. Transcript levels were normalized to transcript level of UBQ5. Bars represent the average \pm SEM of four to six plants of each genotype.

D qRT-PCR analysis of TGA1 transcript levels after (B) SAR experiment of wild-type and tga1 tga4 plants complimented either with empty vector (EV), TGA1 genomic clone (TGA1g) or TGA1 genomic clone carrying mutations in four critical cysteine residues (TGA1gr) under native promoter. Transcript levels were normalized to transcript level of UBQ5. Bars represent the average \pm SEM of five to six plants of each treatment.

Statistical analysis was performed using two-way ANOVA followed by Bonferroni's post-hoc test. Lowercase letters indicate significant differences $(P<0.05)$ between mock-pretreated samples; uppercase letters indicate significant differences $(P<0.05)$ between $(C)$ SA-treated or (D) Psm-infected samples. EV-empty vector, TGA1-TGA1 genomic clone, TGA1gr-TGA1 genomic clone with four cysteines mutated, mock-water (A, C) or magnesium chloride (B, D), SA-salicylic acid, PsmPseudomonas syringae. Experiment under $\mathbf{A}$ was performed by Dyari Mohammed. 


\subsection{Transiently expressed TGA1, TGA2 and SARD1 activate DLO1 promoter in Arabidopsis mesophyll protoplasts}

DLO1 gene, encoding for an enzyme involved in SA catabolism, showed strong TGA1/TGA4 dependence after both SA and Psm treatment. The promoter of this gene contains two TGAbinding sites (i) an extended C-box element and (ii) an A-box element, making it an interesting potential direct target of TGA1. Additionally, the promoter of DLO1 contains two SARD1binding sites. The regulation of DLO1 seems to be rather complicated, because the gene expression was reduced in the absence of NPR1, TGA2/TGA5/TGA6 and SARD1. Therefore, we were interested to unravel the regulation of this gene. To do so, we decided to use transient assay in Arabidopsis mesophyll protoplasts.

For this experiment, we generated reporter construct expressing firefly luciferase ( $L L U C)$ gene under DLO1 (DLO1:fLUC) promoter region 1849 base pairs upstream of the start codon, including the start codon, as shown in Figure 19. Also, we generated three effector plasmids, namely TGA1, TGA2 and SARD1 under the control of UBQ10 (UBIQUITIN10) promoter with the triple HA and streptavidin tag on $C$ terminal site. We used an empty vector expressing $\mathrm{HA}$ under UBQ10 promoter as a control of background promoter activity and Renilla luciferase $(r L U C)$ to normalize for transformation efficiency.

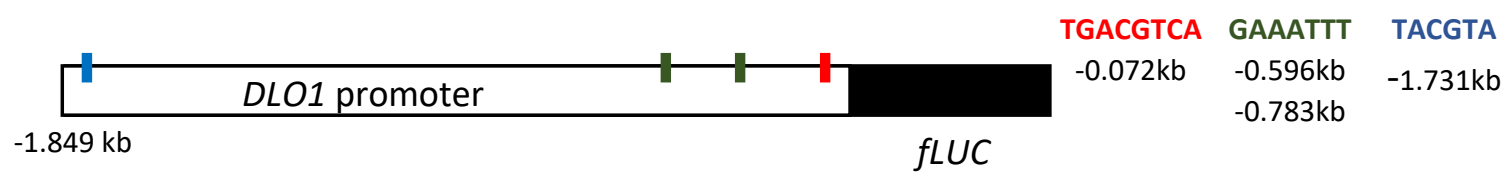

Figure 19 Scheme of the DLO1 promoter reporter construct for luciferase reporter assay.

Sequence of 1.849 kilobases upstream of the start codon including the start codon of DLO1 promoter was cloned into the destination vector to obtain plasmids with $f L U C$ (firefly luciferase) expressed under DLO1 promoter.

TGA-binding sites, an extended C-box element (TGACG) is shown in red and an A-box element (TACGTA) in blue. SARD1-binding motif (GAAATTT) is shown in green. Position upstream of TSS (Transcription Start Site of the corresponding binding motif is indicated on the right side. 


\subsubsection{DLO1 expression is reduced in mutants of clade I and clade II TGA transcription factors}

We used tga1 tga 2 tga 4 tga 5 tga6 mutant to address the role of clade I and clade II TGAs in DLO1 promoter activation. In order to address DLO1 expression in this genotype, wild-type and mutant plants were treated for eight hours either with water or salicylic acid. Expression levels of DLO1 were determined by qRT-PCR. DLO1 gene expression was significantly reduced in tga1 tga2 tga4 tga5 tga6 mutant comparing to the wild-type plants (Figure 20). Therefore, the pentuple tga 1 tga $2 \operatorname{tga} 4 \operatorname{tga} 5 \operatorname{tga} 6$ mutant can be used to address the influence of TGA1 and TGA2 on DLO1 promoter activity.

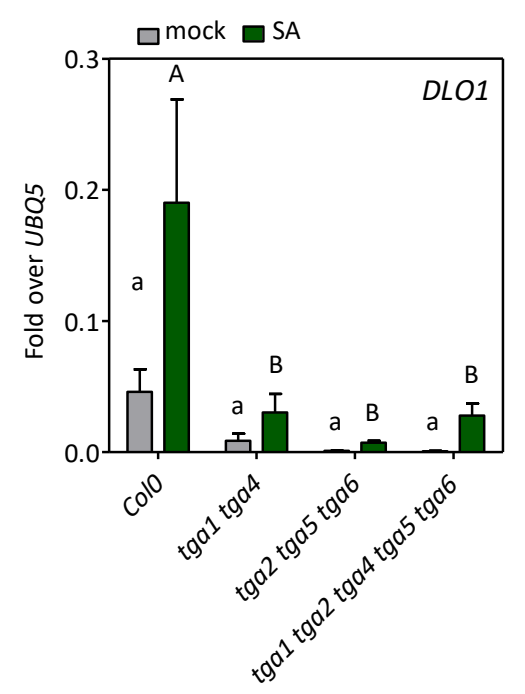

Figure 20 DLO1 expression is dependent on both clade I and clade II TGA transcription factors.

qRT-PCR analysis of DLO1 transcript levels after SA treatment of wild-type and tga1 tga4, tga1 tga5 tga 6 and $n p r 1$ plants. Four-week-old plants were sprayed either with mock or $1 \mathrm{mM} \mathrm{SA}$ at $1 \mathrm{~h}$ after the subjective dawn and further incubated for $8 \mathrm{~h}$. Transcript levels were normalized to transcript level of UBQ5. Bars represent the average \pm SEM of three to five plants of each genotype. Experiment was repeated once with similar results. All data shown here is from the same experiment.

Statistical analysis was performed using two-way ANOVA followed by Bonferroni's post-hoc. Lowercase letters indicate significant differences $(P<0.05)$ between mock-treated samples; uppercase letters indicate significant differences $(P<0.05)$ between SA-treated samples. mock-water, SA-salicylic acid.

3.6.2 TGA1 activates DLO1 promoter in the mutant of clade I and clade II TGA transcription factors

Arabidopsis mesophyll protoplasts were transformed with equal amounts of the effector and the reporter plasmids. Approximately 20 hours after transformation, reporter gene activity 
was measured using the Dual-Luciferase Reporter (DLR ${ }^{T M}$ ) assay system from Promega following manufacturer's instructions. In this experiment, we used TGA1, TGA2 and SARD1 as effectors (indicated in Figure 21A) and DLO1:fLUC as reporter. As showed in Figure 21A, we detected increase in fLUC/fLUC ratio comparing to the empty vector control only when TGA1 was added as an effector. When TGA2 or SARD1 were added there was no significant difference to the empty vector control (HA). Addition of either TGA2 or SARD1 or a combination of both to the TGA1 effector, did not significantly increase fLUC/rLUC ratio of the single TGA1 effector (Figure 21A).

It is known that TGA2 does not contain activation domain, therefore the data for TGA2 was not unexpected. However, we were intrigued that the addition of SARD1 did not lead to a stronger induction. So we analyzed SARD1 levels in the tga1 tga2 tga4 tga5 tga6 mutant plants after SA treatment and they were not significantly different from the wild-type plants (Figure 21B). We assumed that the stress levels caused by the protoplast isolation were comparable to the SA treatment. Therefore, it was plausible to think that SARD1 protein levels in protoplast were very high from the beginning and the addition of SARD1 could not activate DLO1 promoter any further.

A

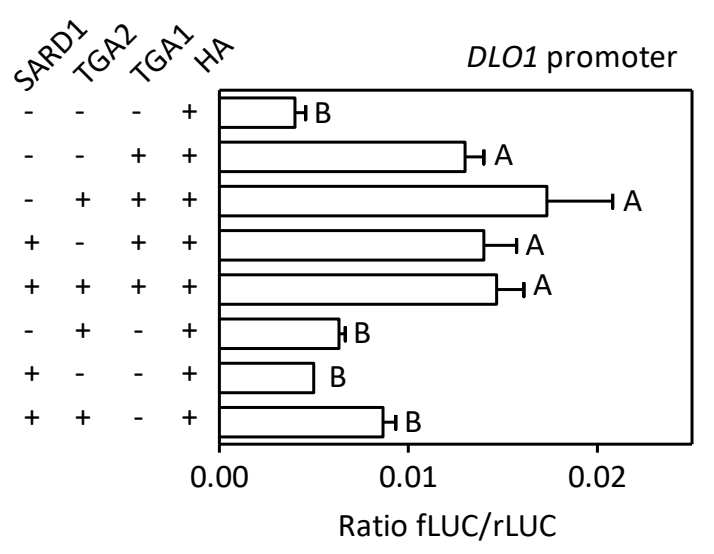

B

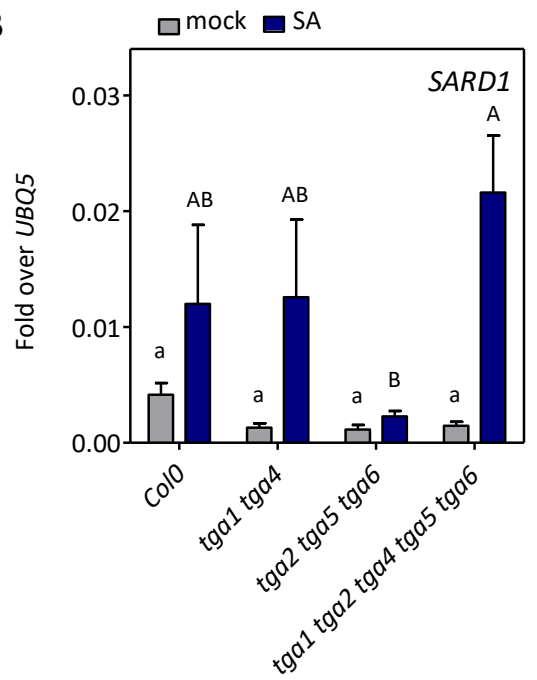

Figure $\mathbf{2 1}$ TGA1 activates DLO1 promoter in Arabidopsis mesophyll protoplasts.

A Arabidopsis mesophyll protoplasts of tga1 tga2 tga 4 tga 5 tga 6 mutant genotype were transfected with equal amounts of effectors (TGA1, TGA2, SARD1, HA) together with reporter plasmid DLO1:fLUC. Approximately 20 hours after transformation, reporter gene activity was measured using the DualLuciferase Reporter (DLR ${ }^{\mathrm{TM}}$ ) assay system from Promega following manufacturer's instructions.

B qRT-PCR analysis of SARD1 transcript levels after SA treatment of wild-type and tga1 tga4, tga1 tga5 tga 6 and $n p r 1$ plants. Four-week-old plants were sprayed either with mock or $1 \mathrm{mM} \mathrm{SA}$ at $1 \mathrm{~h}$ after the 
subjective dawn and further incubated for $8 \mathrm{~h}$. Transcript levels were normalized to transcript level of $U B Q 5$. Bars represent the average \pm SEM of three to five plants of each genotype. Experiment was repeated once with similar results. All data shown here are from the same experiment.

Statistical analysis was performed using one-way (A) or (B) two-way ANOVA followed by Bonferroni's post-hoc test. For (A) + or - indicates whether the effector (name written at the top of each column) was transformed to protoplasts. For $(B)$ lowercase letters indicate significant differences $(P<0.05)$ between mock-treated samples; uppercase letters indicate significant differences $(P<0.05)$ between SA-treated samples. mock-water, SA-salicylic acid.

\subsubsection{TGA1, TGA2 and SARD1 activate DLO1 promoter in the mutant of clade II TGA transcription factors}

Next, we wanted to address the importance of SARD1 for DLO1 promoter activity. Because of the low levels of SARD1 and DLO1 after the SA treatment in tga 2 tga5 tga6 mutant, we used this genotype for the preparation of mesophyll protoplast (Figure 20, Figure 21B). As a control, we transformed protoplasts with TGA1, TGA2 and a combination of TGA1 and SARD1 effectors. The experiment was performed as described in the previous paragraph. SARD1 as effector significantly increased $\mathrm{fLUC/rLUC}$ ratio comparing to the HA control. Interestingly, in this background, both TGA1 and TGA2 were able to activate DLO1 promoter (Figure 22).

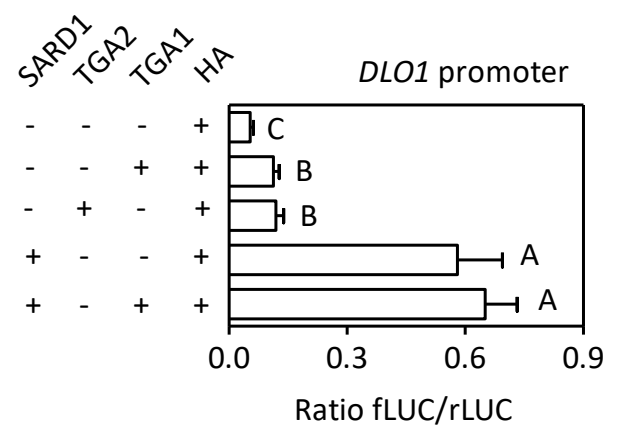

Figure 22 TGA1, TGA2 and SARD1 activate DLO1 promoter expression in Arabidopsis mesophyll protoplasts.

Arabidopsis mesophyll protoplasts of the tga $2 \operatorname{tga} 5 \operatorname{tg} a 6$ mutant genotype were transfected with equal amounts of effectors (HA, TGA1, TGA2, SARD1) together with reporter plasmid DLO1:fLUC. Approximately 20 hours after transformation, reporter gene activity was measured using the DualLuciferase Reporter (DLR ${ }^{\mathrm{TM}}$ ) assay system from Promega following manufacturer's instructions.

Statistical analysis was performed using one-way ANOVA and Bonferroni's post-hoc test. Samples with a significant difference at $P<0.01$ are indicated with different letters. + or - indicates whether the effector (name written at the top of each column) was transfected to the protoplasts. This experiment was performed by Anna Herman. 


\subsubsection{Mutation in the C-box but not in the A-box element abolishes TGA1- and TGA2-induced} DLO1 promoter activity

TGA1 was shown to bind extended to the extended C-box element (TGACG) of SARD1 promoter (Sun et al, 2017) and the A-box element (TACGTA) of ATH1 promoter (Wang et al, 2019). As illustrated in Figure 19, DLO1 promoter contains both motifs and we wanted to find out which of the two was important for TGA1-mediated induction. To do so, we mutated either the A-box or the C-box element of DLO1 promoter and fused it to $f L U C$ gene. Because TGA1-induced DLO1 expression was not very high when native TGA1 was used, we prepared TGA1-VP construct where genomic TGA1 was fused with a strong transactivation domain of herpes simplex virus protein 16 (VP16). The same construct was prepared for SARD1 and TGA2. As a control plasmid, HA-VP was used.

Arabidopsis mesophyll protoplasts of wild-type plants were transformed with equal amounts of HA-VP or TGA1-VP, TGA2-VP and SARD1-VP effector and either wild-type DLO1:fLUC, A-box mutated DLO1:fLUC or C-box box mutated DLO1:fLUC reporter plasmid. Approximately 20 hours after transformation, reporter gene activity was measured using the Dual-Luciferase Reporter (DLR ${ }^{\mathrm{TM}}$ ) assay. First, we observed a strong induction of DLO1 promoter with TGA1VP and TGA2-VP comparing to the HA control (Figure 23A). This was not the case for SARD1VP (Figure 23A). Mutation of the A-box element (TACGTA to TTTTTA) did not influence TGA1and TGA2-activated DLO1 expression and it behaved as the wild-type promoter (Figure 23B). Interestingly, mutation of the C-box element (TGACGT to TTTTTT) completely abolished TGA1and TGA2-mediated DLO1 promoter induction (Figure 23B). Thus, TGA1 and TGA2 required TGACGT sequence of DLO1 promoter in order to activate its transcription. The data for SARD1 is not conclusive because SARD1-VP was not strong inducer of DLO1 promoter (Figure 23A). 
A

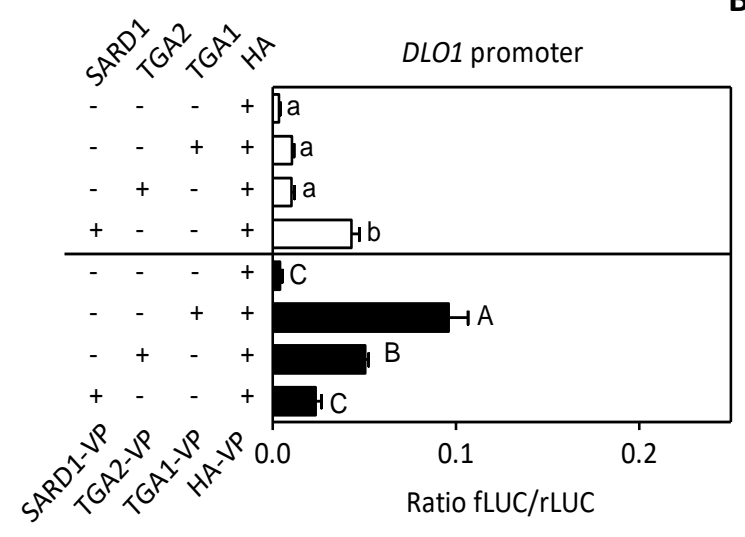

B

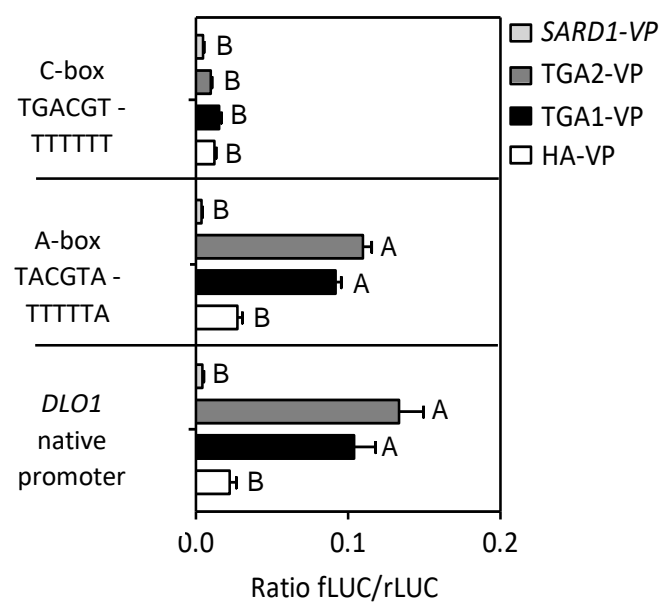

Figure 23 TGA1 needs functional C-box to activate DLO1 promoter expression in Arabidopsis mesophyll protoplasts.

Arabidopsis mesophyll protoplasts of wild-type plants were transfected with equal amounts of effectors (HA, TGA1, HA-VP, TGA1-VP) together with a reporter plasmid containing firefly luciferase under native DLO1 promoter (A) or mutated DLO1 promoter (B). Mutation was introduced either at the A-box element (TACGTA - TTTTTA) or the C-box element (TGACGT - TTTTTT). Approximately 20 hours after transformation, reporter gene activity was measured using the Dual-Luciferase Reporter $\left(D_{L R}{ }^{\mathrm{TM}}\right)$ assay system from Promega following manufacturer's instructions.

Statistical analysis was performed using two-way ANOVA and Bonferroni's post-hoc test. For (A) lowercase letters indicate significant differences $(P<0.05)$ between samples transfected with the native effector; uppercase letters indicate significant differences $(P<0.05)$ between samples transfected with the VP-tagged effector. + or - indicates whether the effector (name written at the top of each column) was transfected to protoplasts. Mutant constructs of DLO1 promoter and fusions of effectors with the transactivation domain of VP-16 were prepared by Anna Herman. This experiment was performed by Anna Herman. 


\subsection{Highly expressed CC-type glutaredoxins are not responsible for the low expression of SA-inducible genes in tga1 tga4 mutant}

3.7.1 Expression of glutaredoxin-like proteins ROXY11, ROXY12, ROXY13, ROXY14 and ROXY15 was increased in sid2 tga1 tga4 mutant

RNA sequencing data revealed 777 genes which were upregulated in sid 2 tga1 tga4 mutant compared to sid2 mutant independent of SA treatment (fold change ( $\log F C \geq-1), p<0.05$ ) (Figure 24A). In this group, we found thirteen CC-type glutaredoxins ROXYs (Figure 24B). All thirteen of them have ALWL motif on their C-terminal end, which was previously reported to be crucial for repression of TGA2/TGA5/TGA6 (Zander et al, 2014, Uhrig et al, 2016).

Therefore, we hypothesized that the highly expressed ROXYs act as repressors of TGA2/TGA5/TGA6 activity in tga1 tga4 mutant, which finally results in a lower expression of marker genes ( $D L O 1, B G L 2)$. Considering that the ROXYS are constantly upregulated in tga1 tga4 mutant, there is no de-repression of clade II TGA factors and thus the full induction is never achieved. The notion that DLO1 and BGL2 had lower induction in the tga 2 tga5 tga6 mutant, further supports the hypothesis that these TGA factors are responsible for the SAdependent transcriptional activation.

A

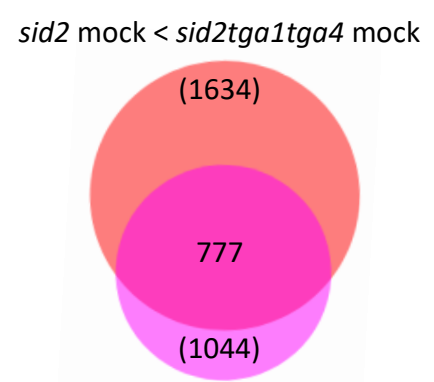

sid2 SA < sid2tga1tga4 SA
B

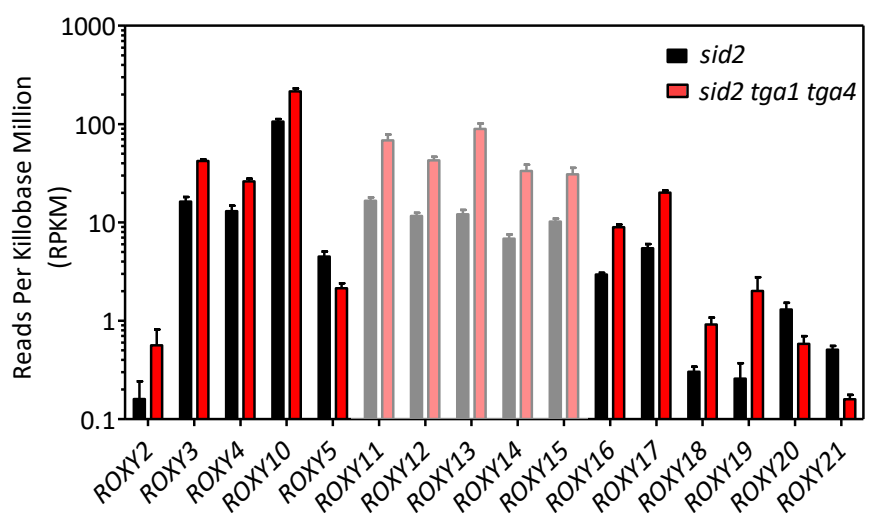

Figure 24 Expression of ROXY genes is increased in sid2 tga1 tga4 mutant

A Venn diagram of genes which are more expressed in sid2 tga1 tga4 mutant than in sid2 after eight hours of mock and SA treatment.

B Expression levels of sixteen ROXY genes which contain ALWL sequence on C terminal end in sid2 and sid2 tga 1 tga 4 mutant. For the simplicity of the graph, only mock treated samples are shown. Plants were sprayed with mock at $1 \mathrm{~h}$ after the subjective dawn and further incubated for $8 \mathrm{~h}$. Transcriptome 
analysis was performed using Illumina sequencing. Bars represent the average of Reads per Kilobase Million (RPKM) \pm SEM of four plants of each genotype. Expression of ROXY11-15 genes is shown in lighter color.

Statistical analysis was performed using RobiNA software with fold change (log2 FC $>1)$ and $P<0.05$. RPKM - Reads Per Kilobase Million.

\subsubsection{CRISPR-Cas9 was used to knock out a gene cluster}

ROXY11, ROXY12, ROXY13, ROXY14 and ROXY15 (from now on ROXY11-15) were one of the most highly expressed amongst the ROXYs (Figure 25B). These genes are arranged in a cluster on chromosome four of Arabidopsis thaliana. Thus, we could use CRISPR-Cas9 to induce deletion of the entire cluster to obtain roxy11-15 mutant in tga1 tga4 mutant background.

To do so, we designed three types of oligonucleotides, named A, B and C, each targeting different sets of genes from the cluster. The major goal of this CRISPR-Cas9 approach was to cause the deletion of five genes with oligonucleotides targeting the outermost genes ROXY15 and ROXY11 (oligo A). As a backup plan for deletion, we also targeted either ROXY15, ROXY14 or ROXY11 (oligo B) or all five genes (oligo C) (Table 20). Each delivery vector consisted of a Green-Fluorescent Protein (GFP) under the seed-specific promoter as a selection marker, a CRISPR-associated 9 (Cas9) gene under the egg-cell specific promoter and carried a combination of guide RNAs (AB, AC, BC, CB, CA, BA).

Table 20 Oligonucleotides, which were used to guide Cas9, targeted different ROXY11-15 genes

\begin{tabular}{l|l|l|l|l|l} 
gene & ROXY15 & ROXY14 & ROXY13 & ROXY12 & ROXY11 \\
\hline oligonucleotide & A, B, C & B, C & C & C & A, B, C
\end{tabular}

Plants were transformed using the floral dip method (Clough \& Bent, 1998). T1 generation seeds were visualized under a fluorescence microscope. As the vector carried a GFP gene under seed-specific promoter, fluorescence was a sign of T-DNA presence. Only the glowing seeds, were planted on the soil. Genomic DNA of five T1 plants was pooled and used for PCR genotyping with primers for outermost genes (ROXY11 and ROXY15). Only if the deletion occurred, polymerase would be able to amplify the area otherwise there would be no product due to the length of the amplified region. PCR products were separated by agarose gel electrophoresis and visualized under UV light. Plants from the pools which yielded a PCR fragment of the expected size were allowed to set seeds. Seeds of T2 were again inspected 


\section{Results}

under a microscope. In this step, we wanted to find stable lines lacking the nuclease, so only the seeds without T-DNA (non-glowing seeds) were planted on the soil. Genomic DNA was extracted from the leaves and further analyzed with PCR using the outermost primers. In a case of a successful PCR, resulting in a fragment of the expected size, new PCRs with gene specific primers (ROXY11, ROXY12, ROXY13, ROXY14 and ROXY15) were performed. Products were sent for sequencing and analysis of sequences reviled whether the plant is homo-or heterozygous.

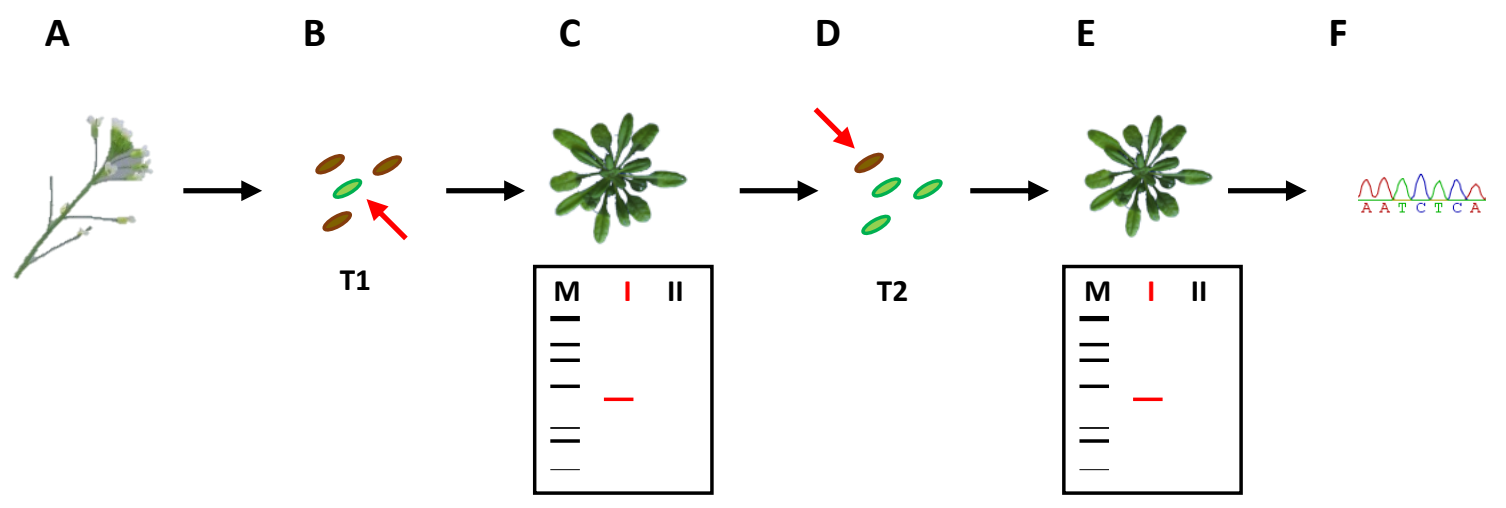

Figure 25 Timeline of CRIPRS-Cas9 transformation and mutagenesis.

A Flowering plant was transformed using Agrobacterium vector system.

B Vector delivered GFP under seed-specific promoter which enabled selection using a fluorescence microscope. Only fluorescent seeds were planted on soil (red arrow).

C Genomic DNA of these plants was analyzed via PCR using outermost primers. Only if a deletion had occurred, polymerase was be able to amplify the fragment. Plants which yielded a product of expected size were allowed to set seeds.

D T2 generation was again selected using a fluorescence microscope, only this time the seeds without GFP, which do not contain active T-DNA construct, were selected (red arrow).

E Genomic DNA of these plants was again template for PCR with outermost primers.

F PCR products were sent for sequencing and homozygous plants were allowed to set seeds which will be used in further experiments. $\mathrm{M}$ - marker.

Finally, we obtained roxy11-15 and roxy11-15 tga1 tga4 mutant using oligonucleotide combination $B C$ and AC. roxy11-15 tga1 tga4 had a 26 base pair deletion upstream of Protospacer Adjacent Motif (PAM) sequence of oligonucleotide B and roxy11-15 had a 5 base pair insertion upstream of PAM sequence of oligonucleotide A (Supplementary Figure 7). 


\subsubsection{Elevated ROXY11-15 levels in tga1 tga4 mutant do not repress TGA1/TGA4 dependent} genes after SA treatment

According to our hypothesis, ROXY11-15 are repressors of TGA2/TGA5/TGA6 in tga1 tga4 mutant. Therefore, the loss of ROXY11-15 in the tga1 tga4 mutant background would restore wild-type-like levels of target genes, such as DLO1. To test this hypothesis, we generated two types of mutants, a roxy11-15 and a roxy11-15 tga1 tga4 (heptuple) mutant.

For the experiment, four-week-old plants were treated either with water as a control or SA and samples were collected for analysis. As a readout, DLO1 levels were analyzed using qRTPCR. Following the SA treatment, there was no significant difference in DLO1 expression between tga1 tga4 mutant and roxy11-15 tga1 tga4 indicating that highly expressed ROX1115 are not responsible for lower expression of DLO1 in tga1 tga4 mutant background (Figure 26).
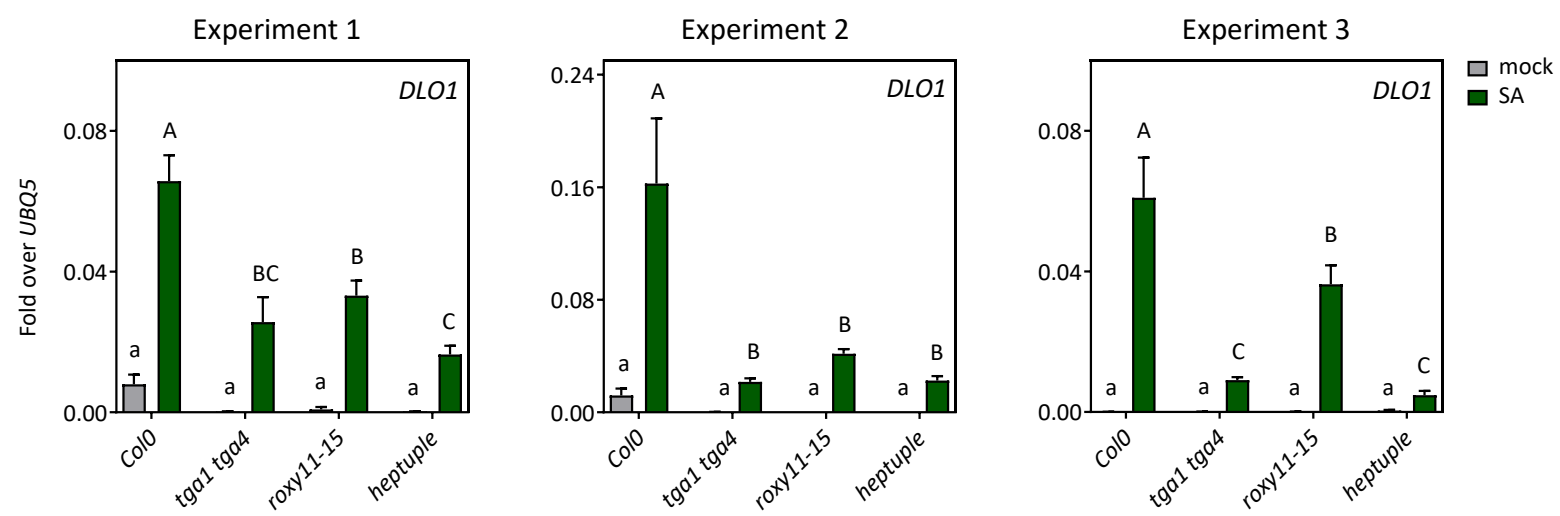

Figure 26 Low expression of DLO1 in tga1 tga4 mutant is not a consequence of high ROXY11-15 levels.

qRT-PCR analysis of DLO1 transcript levels after SA treatment of wild-type and tga1 tga4, roxy11-15 and heptuple mutant plants. Four-week-old plants were sprayed either with mock or 1mM SA at $1 \mathrm{~h}$ after the subjective dawn and further incubated for $8 \mathrm{~h}$. Transcript levels were normalized to transcript level of UBQ5. Bars represent the average \pm SEM of five to six plants of each genotype. Control plants, $\mathrm{Col}-0$ and tga 1 tga 4 mutant, were outcrossed during segregation step.

Statistical analysis was performed using two-way ANOVA followed by Bonferroni's post-hoc test. Lowercase letters indicate significant differences $(P<0.05)$ between mock-treated samples; uppercase letters indicate significant differences $(P<0.05)$ between SA-treated samples. mock-water, SA-salicylic acid. 
Interestingly, roxy11-15 mutant displayed lower expression of DLO1 as compared to wild-type plants (Figure 26-different experiments). ROXY11-15 seem not to be negative regulators of TGA1/TGA4-dependent genes but rather positive ones. Since there is no additive effect in the heptuple mutant as compared to tga1 tga4 mutant, it is plausible to hypothesize that they all work in the same cascade.

\subsubsection{Elevated ROXY11-15 levels in tga1 tga4 mutant are not important for induction of} TGA1/TGA4-dependent genes upon pathogen attack in SAR experiment

Additional experiment to test the hypothesis that ROX11-15 act as repressors of TGA2/TGA5/TGA6 in the tga1 tga4 mutant background, was induction of target genes in upon SAR. For this experiment, plants were either pretreated with Psm bacteria or mock solution. The second infection was performed two days after with Psm and samples were collected eight hours after treatment. SARD1, DLO1 and BGL2 levels were analyzed using qRT-PCR. Transcription levels of marker genes were drastically decreased in tga1 tga4 mutant, as seen before. There was no significant difference between $\operatorname{tga} 1$ tga4 and the heptuple mutant (Figure 27), as seen in the previous experiment with SA-treated plants. Once again, roxy11-15 mutant had lower levels of target genes than wild-type plants, supporting the hypothesis that ROXY11-15 could be positive regulators of TGA1/TGA4-dependent genes (Figure 27).

A

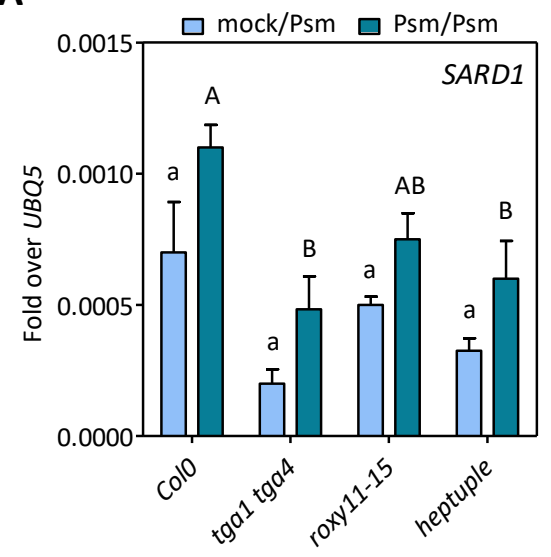

B

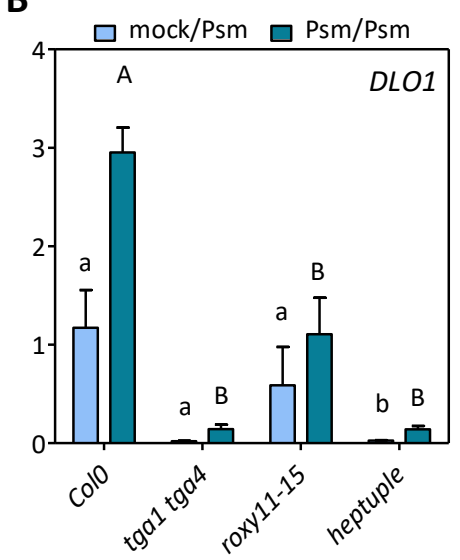

C

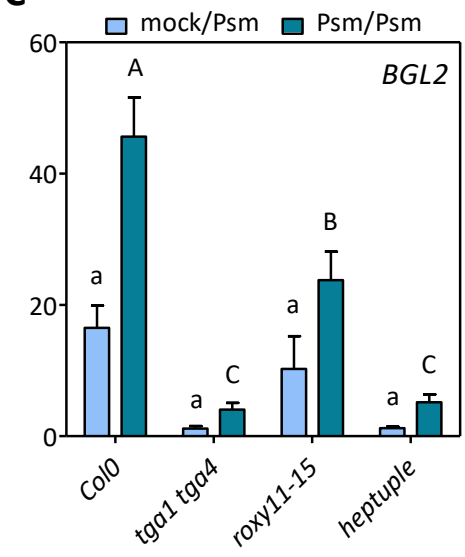

Figure 27 Lower expression of target genes in tga1 tga 4 mutant after pathogen infection is not a consequence of high ROXY11-15 levels.

qRT-PCR analysis of SARD1, DLO1 and BGL2 transcript levels after Pseudomonas infection of wild-type, tga1 tga4, roxy11-15 and heptuple mutant plants. Three older leaves of five-week-old plants were mock or Pseudomonas treated at $1 \mathrm{~h}$ after the subjective dawn on day one of the infection. After two 


\section{Results}

days, three younger upper leaves were untreated, mock or Pseudomonas treated. Three upper leaves were collected $8 \mathrm{~h}$ after secondary treatment and RNA was extracted. Transcript levels were normalized to transcript level of UBQ5. Bars represent the average \pm SEM of four to six plants of each treatment.

Statistical analysis was performed using two-way ANOVA followed by Bonferroni's post-hoc test. Lowercase letters indicate significant differences $(P<0.05)$ between mock-pretreated samples; uppercase letters indicate significant differences $(P<0.05)$ between Psm-infected samples. mockmagnesium chloride pretreated, Psm-Pseudomonas infected. This experiment was performed by Aswin Nair.

\subsubsection{Elevated ROXY11-15 levels in tga1 tga4 mutant are not important for susceptibility of tga1 tga4 mutant}

The double tga1 tga 4 mutant is reportedly more susceptible than wild-type plants after infection by virulent or avirulent strain of Pseudomonas syringae (Kesarwani et al, 2005, Shearer et al, 2012, Muthreich thesis, 2016, Sun et al, 2017). We were interested to see if ROXY11-15 genes are involved in susceptibility of tga1 tga 4 mutant. Since weak susceptibility differences between wild-type and mutants are better visible when less virulent pathogens are used, we decided to use Pseudomonas syringae pathovar tomato DC3000 strain which lacks two virulence genes avrPto/avrPtoB (PtoLavrPto/PtoB) (Lin and Martin, 2005). Plants were syringe infiltrated with a suspension of Pto $\triangle a v r P t o / P t o B\left(\mathrm{OD}_{600}=0.001\right)$. Three days after the infection, bacteria were retrieved from the infected leaves and bacterial growth was determined by the number of Colony-Forming Units (CFU). Increased susceptibility of tga1 tga4 mutant compared to wild-type was once more observed, however there was no difference between susceptibility of tga1 tga 4 mutant and the heptuple mutant. Furthermore, roxy11-15 mutant showed increase in susceptibility in one experiment but not as strong as tga1 tga4 and the heptuple mutant (Figure 28, experiment II). 


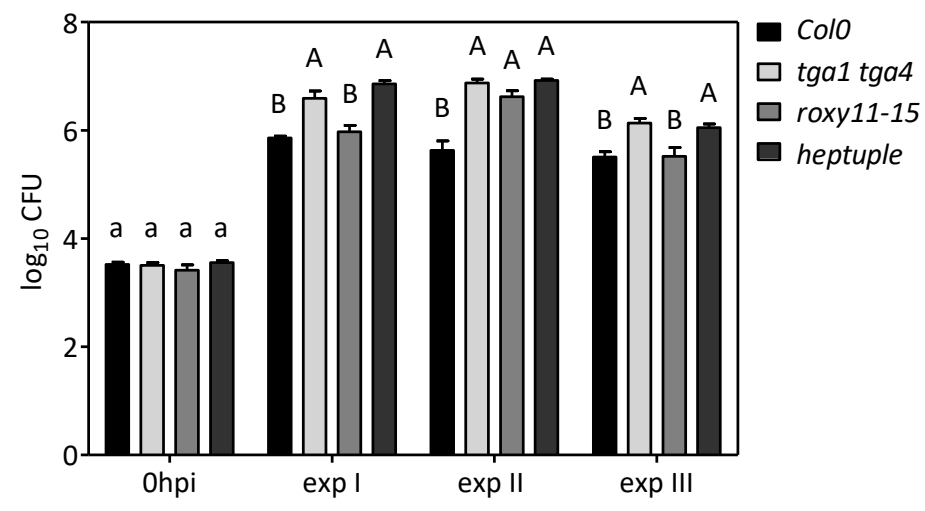

Figure 28 Heptuple mutant has the same susceptible to Pseudomonas syringae pv. tomato DC3000 $\triangle a v r$ to/avrPtoB as tga1 tga 4 mutant.

Three older leaves of five-week-old plants were syringe infiltrated with Pto $\Delta$ avrPto/PtoB solution of $\mathrm{OD}_{600}$ of 0.002 . Three leaves were collected immediately and 3 discs from each leaf were collected in $10 \mathrm{mM}$ magnesium chloride solution to serve as Ohpi samples. Other samples were collected similarly 3 days after infection. Discs were ground using metallic beads and suspension of bacteria was diluted in a series of dilution. Bacterial dilutions were plated on King's B agar plates containing respective selective antibiotics. Two days after incubation at $29{ }^{\circ} \mathrm{C}$, bacterial colonies were counted and CFU (Colony Forming Units) was calculated. Bars represent average CFU of bacteria \pm SEM from four to seven biological replicates

Experiment was repeated three times and all results are displayed in the graph. Statistical analysis was performed using one-way ANOVA and Bonferroni's post-hoc test for all the experiments separately. Lowercase letters indicate significant differences $(P<0.05)$ between mock-treated samples; uppercase letters indicate significant differences $(\mathrm{P}<0.05)$ between Pto $\Delta$ avrPto/PtoB-infected samples. CFUColony-Forming Units, hpi-hour post infection, exp-experiment, Pto $\Delta$ avrPto/PtoB-Pseudomonas syringae pv. tomato DC3000 $\triangle$ avrPto/avrPtoB. 


\subsection{ROXY9-modulated repression of TGA1 is not released in roxy6 roxy7 roxy8 roxy9 mutant}

\subsubsection{Overexpression of ROXY9 mimics tga1 tga4 mutant after SA treatment}

Ectopically expressed ROXY8 and ROXY9, which do not contain ALWL motif at the C terminus, repress the activity of TGA1/TGA4 in hyponastic growth (Li et al, 2018). We were interested to see if ROXY9 can repress TGA1/TGA4-dependent DLO1, BLG2 and PR1 induction after SA treatment. Therefore, we used SA spraying experiment under the conditions as explained above and detected the levels of the marker genes using QRT-PCR. Indeed, ROXY9 overexpression line showed decrease in DLO1, BGL2 and PR1 induction and this was not significantly different from the induction in the tga1 tga4 mutant (Figure 29).
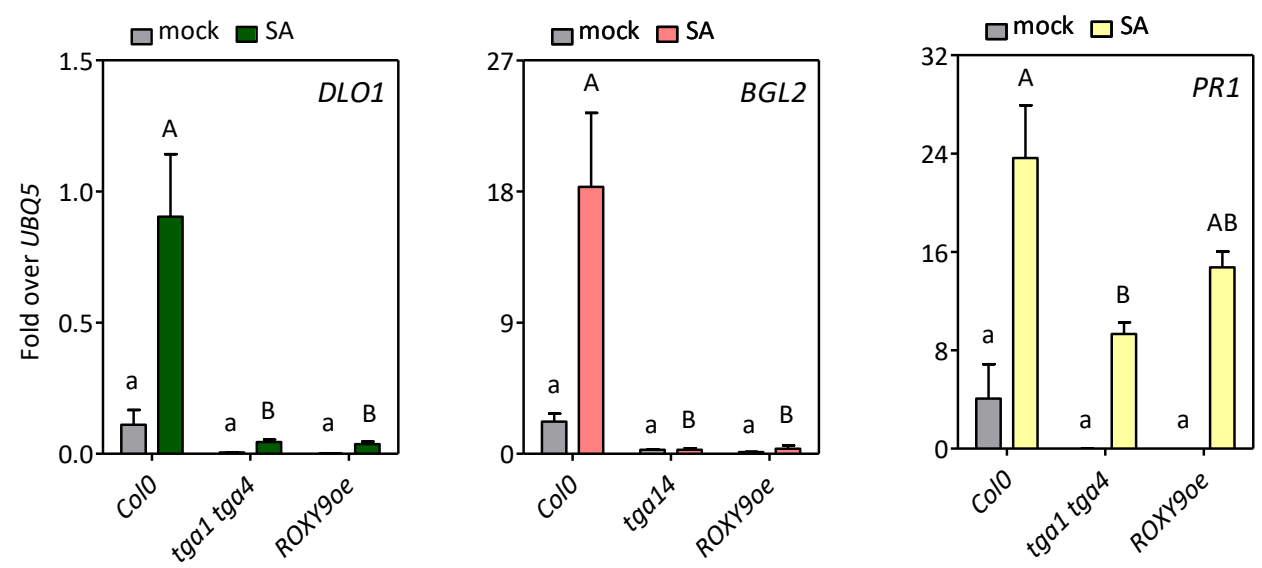

Figure 29 TGA1/TGA4-mediated gene induction after SA is repressed in ROXY9 overexpression line.

qRT-PCR analysis of DLO1, BGL2 and PR1 transcript levels after SA treatment. Four-week-old plants were sprayed either with mock or $1 \mathrm{mM} \mathrm{SA}$ at $1 \mathrm{~h}$ after the subjective dawn and further incubated for $8 \mathrm{~h}$. Transcript levels were normalized to transcript level of UBQ5. Bars represent the average \pm SEM of five to six plants of each genotype. Experiment was performed once.

Statistical analysis was performed using two-way ANOVA followed by Bonferroni's post-hoc test. Lowercase letters indicate significant differences $(P<0.05)$ between mock-treated samples; uppercase letters indicate significant differences $(P<0.05)$ between SA-treated samples. mock-water, SA-salicylic acid.

\subsubsection{Using CRISPR-Cas9 to knock-out ROXY6 and ROXY7 in roxy9 mutant background}

ROXY9 protein belongs to a group of the four CC-type glutaredoxins which do not contain ALWL motif at their $C$ terminal site. The proteins share very high homology so there is a 
possible redundancy between the four proteins. In order to address the relationship between these ROXs and clade I TGA transcription factors, we decided to generate a mutant of the four ROXYs, namely ROXYG, ROXY7, ROXY8 and ROXY9. Out of the four, I concentrated on mutating ROXY6 and ROXY7 genes using CRISPR-Cas9 technology.

ROXYG and ROXY7 are situated on different chromosomes, so we could not use the same approach as with the ROXY11-15 mutant. Instead of aiming for a big deletion, we wanted to induce a small insertion or a deletion which would lead to a premature stop codon. Oligonucleotides we used in this experiment were designed to target gene the regions around the putative active site motif. Each vector used for the transformation consisted of a BASTA gene cassette as a selection marker gene, a GFP gene under a seed-specific promoter, a Cas 9 gene under an egg-cell specific promoter and carried a combination of guide RNA targeting ROXYG and ROXY7.

Wild-type and roxy9 mutant plants were transformed using the floral dip method (Clough \& Bent, 1998). T1 generation of plants was selected with BASTA. Genomic DNA of five T1 plants which were BASTA-resistant was pooled and used for PCR genotyping with primers for ROXYG and ROXY7 genes. The products of reaction were then analyzed by T7 endonuclease 1 (T7E1) assay. The enzyme recognizes and cleaves heteroduplex DNA that is formed due to the heterozygosity of gene alleles. If a DNA fragment consists of two different single strands (e.g. a wild-type allele and a mutated fragment) it will be cut by T7E1 which will result in two fragments. If the DNA fragment consists of identical single-strands, which can be either wildtype or mutated, it will not form a heteroduplex and will not be cut. Therefore, the heterozygous plants will have two fragments when products of T7E1 assay are separated by agarose gel electrophoresis and visualized under UV-light. Plants from the pools which yielded two fragments after T7E1 assay were allowed to set seeds. Seeds of T2 were inspected under a fluorescence microscope. The vector carried a GFP under a seed-specific promoter and fluorescence was a sign of T-DNA presence. In this step, we wanted to find stabile lines, so only the seeds without T-DNA (non-glowing seeds) were planted on the soil. Genomic DNA was extracted from the leaves further analyzed via PCR and T7E1 assay. Mutants which were homozygous for a mutation in either gene were allowed to set seeds. Using this approach, we were able to generate roxy6 and roxy7 roxy9 mutants. Unfortunately, roxy6 roxy7 roxy9 mutant was not generated in this step. Therefore, roxy 6 and roxy 7 roxy 9 mutants were 
crossed to obtain the higher order mutant. The roxy8 mutant was obtained in roxy9 background using a similar approach by Katrin Treffon. Finally, the roxy 6 roxy7 roxy8 roxy 9 (from now on roxy6789) mutant was obtained through cross of roxy6 roxy 7 roxy9 and roxy8 roxy9 mutants (Supplementary Figure 8).

\subsubsection{ROXY9-mediated repression of TGA1 is not released in roxy6 roxy7 roxy8 roxy9 mutant} after SA treatment

Since ROXY9 overexpression mimics tga1 tga4 mutant, we expected that the loss of ROXY6-9 will lead to the hyper induction of TGA1/TGA4-regulated genes. We analyzed TGA1/TGA4 target genes which are less induced after SA treatment (DLO1, BGL2, PR1). The SA-spraying experiment was performed as described above. The levels of maker genes were detected using qRT-PCR. Once again, we observed lower induction of the marker genes after the SA treatment in tga1 tga4 mutant and ROXY9 overexpression lines as compared to the wild-type plants. However, the loss ROXY6-9 did not reverse this effect (Figure 30). On contrary, roxy6789 showed a decrease in expression of $D L O 1, B G L 2$ and $P R 1$ comparing to the wild-type plants.
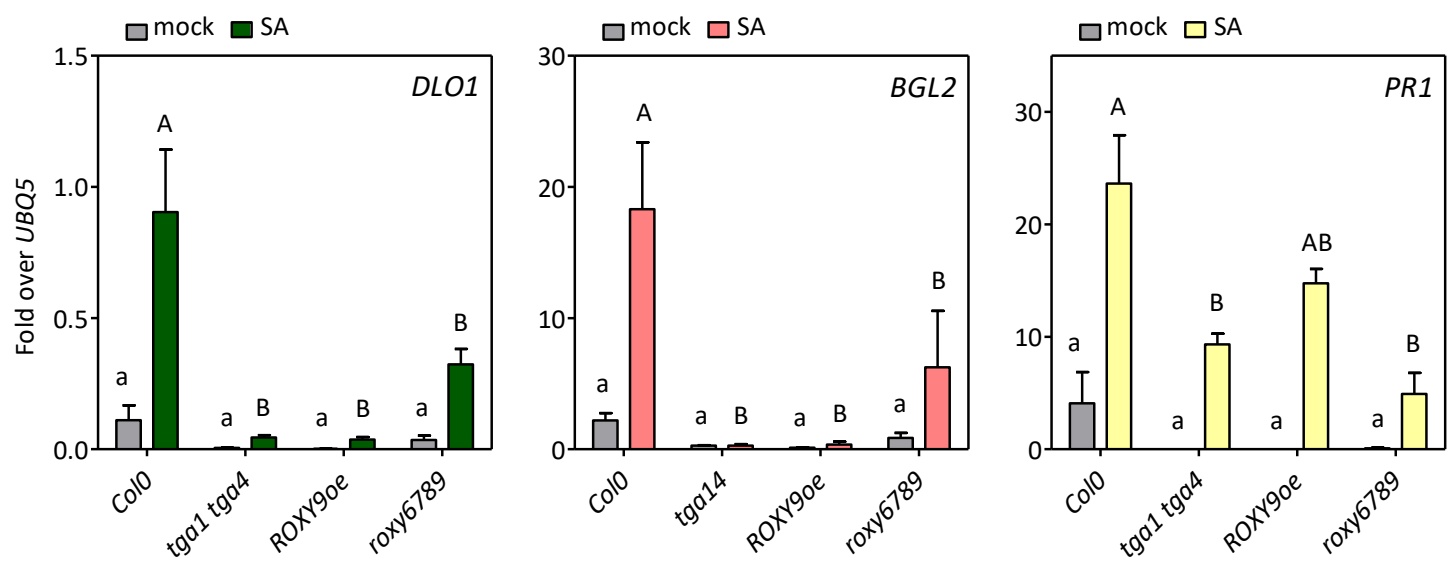

Figure 30 Repression caused by overexpression of ROXY9 is not released in roxy6-9 mutant.

qRT-PCR analysis of DLO1, BGL2 and PR1 transcript levels after SA treatment. Four-week-old plants were sprayed either with mock or $1 \mathrm{mM} \mathrm{SA}$ at $1 \mathrm{~h}$ after the subjective dawn and further incubated for $8 \mathrm{~h}$. Transcript levels were normalized to transcript level of UBQ5. Bars represent the average \pm SEM of five to six plants of each genotype. Experiment was performed once.

Statistical analysis was performed using two-way ANOVA followed by Bonferroni's post-hoc test. Lowercase letters indicate significant differences $(P<0.05)$ between mock-treated samples; uppercase letters indicate significant differences $(P<0.05)$ between SA-treated samples. mock-water, SA-salicylic acid. 


\section{Discussion}

TGA1 and TGA4 form a clade I of basic leucine zipper transcription factors. They are important for basal (Kesarwani et al. 2007) and systemic immunity (Sun et al. 2018). Based on the higher susceptibility of the tga1 tga4 npr1 mutant as compared to the tga1 tga4 and the npr1 mutant, it was concluded that modulation of at least basal immunity by TGA1/TGA4 is independent of NPR1 (Shearer et al. 2012). This finding was somewhat surprising because SA treatment facilitates TGA1-NPR1 interaction (Després et al. 2003). In the uninduced state, TGA1 forms intramolecular disulfide bridge which is reduced upon SA treatment. Because in reduced state TGA1 can interact with NPR1, it was postulated that reduced form is the active form of the protein. Moreover, TGA1 is also shown to be glutathionylated and nitrosylated in vitro (Lindermayr et al. 2010). So far, the importance of TGA1-redox state has not been addressed in vivo.

Here we report on identification of TGA1/TGA4-dependent genes downstream of SA which are part of NPR1- and TGA2/TGA5/TGA6-dependent SA pathway. Moreover, we addressed the role of TGA1-redox state using complementation strategy. The double tga1 tga4 mutant was complemented with either genomic TGA1 (TGA1g) or a TGA1 with four critical cysteines mutated to mimic the reduced form of the protein (TGA1gr). We first addressed the role of cysteine residues after SA treatment. Additional pathogen infection experiments were performed to induce not only accumulation of SA, but also nitric oxide (NO), since both molecules were reported to modify TGA1 protein (Durrant and Dong 2004; Delledonne et al. 1998).

Furthermore, we were interested in known TGA-interaction partners, CC-type glutaredoxins also known as ROXYs. A subgroup of ROXYs, which have ALWL motif on their C-terminal site, can repress TGA2/TGA5/TGA6 function. Because their transcripts were elevated in tga1 tga4 mutant, we postulated that these ROXYs repress TGA5/TGA5/TGA6 in the mutant. To address this hypothesis, we generated mutants of highly expressed ROXY11-15 genes in tga1 tga4 mutant background. Another CC-type glutaredoxin, ROXY9 protein, which does not contain ALWL motif on its C-terminal site, can repress TGA1 and TGA4 protein. In order to better understand interactions between clade I TGA factors and ROXY9, we generated the mutants of ROXY9 and its closest homologues ROXYG, ROXY7 and ROXY8 genes. 


\subsection{TGA1 is modulated by SA but what is its role downstream of SA?}

\subsubsection{Identification of TGA1/TGA4-dependent genes downstream of SA}

Using RNA sequencing strategy, we have identified 207 TGA1/TGA4-dependent genes downstream of SA (Error! Reference source not found.3). Fitting to their role in basal and $s$ ystemic immunity, the group of 207 genes was enriched in gene ontology biological processes "terms responses to bacteria" and "biotic stimulus" (Error! Reference source not found.3). As o pposed to microarray analysis of SA-treated wild-type and tga1 tga4 mutant plants from Shearer et al., we have been able to identify SA-inducible TGA1/TGA4-dependent genes (Shearer et al, 2012). It is possible that different observations are a consequence of different experimental conditions between the two labs.

\subsubsection{TGA1/TGA4-dependent genes are inducible through NPR1-TGA2/TGA5/TGA6 and SARD1/CBP60g cascade}

Because redox change of TGA1 after SA treatment facilitates NPR1-TGA1 interaction, we were interested to see if our marker genes belong to NPR1-dependent genes. Already mentioned study from Shearer and colleagues showed that TGA1 and NPR1 work in reciprocal manner (Shearer et al. 2012). They found that NPR1-dependent genes such as $P R 1$ and $P R 5$ are higher expressed in tga1 tga4 mutant than in wild-type plants after mock and SA treatment. Nevertheless, tga1 tga4 mutant was more susceptible to infection with biotrophic pathogen than the wild-type. This study showed that TGA1/TGA4 and NPR1 work independently because tga1 tga4 npr1 mutant had higher susceptibility than tga1 tga4 and npr1 mutant. Since there was a small subset of genes which was positively regulated by NPR1 and negatively by TGA1/TGA4, they proposed that TGA1/TGA4 act as repressors on these promoters. Upon reduction of disulfide bridge, TGA1 can interact with NPR1 and the repression is released. Because most of the NPR1-dependent genes were not influenced in tga1 tga4 mutant, it is postulated that NPR1 mostly works through other TGA factors.

Interestingly, Kesarwani and colleagues also reported that tga1 tga4 and tga1 single mutant are more susceptible to pathogen, but in their hands, transcript levels of $P R 1$ in tga1 single mutant was lower than in the wild-type plants (Kesarwani et al. 2007). 
Here we showed that TGA1/TGA4-dependent marker genes were also NPR1-dependent, indicating that they work in the same pathway. Moreover, SA-induction was also dependent on TGA2/TGA5/TGA6 and SARD1/CBP60g (Figure 9, Figure 10and Supplementary Figure 4). SA marker genes which were also TGA1/TGA4-dependent as BGL2 and PR1 showed stronger and more reproducible dependency on NPR1-TGA2/TGA5/TGA6 and SARD1/CBP60g than on TGA1/TGA4. Thus, we think that in general, SA induction pathway is established through NPR1-TGA2/TGA5/TGA6 downstream of which SARD1/CBP60g play a role. Importance of TGA1/TGA4 for induction of these genes varied among different experiments and was highly influenced by growth conditions. In contrast to BGL2 and PR1, induction of DLO1 (DOWNEY MILDEW RESISTANCE6-LIKE OXYGENASE1) was reproducibly TGA1/TGA4-dependent. DLO1 encodes for a salicylic acid 3-hydroxylase, an SA-catabolism enzyme (Zhang et al. 2013). Mutant of DLO1 and its closest homologue DMR6 (DOWNY MILDEW RESISTANT6) accumulates higher levels of $S A$ and $P R$ genes and is consequently more resistant to infection by biotrophic pathogen (Zeilmaker et al. 2015). We showed here that DLO1 gene was SA-inducible and therefore it belonged to a negative feedback regulation of SA response. Because of its importance, the regulation of this gene is rather complex. We think there are two modes of regulation (i) basal regulation via TGA1-NPR1 and (ii) SA-inducible regulation which is established on two levels (a) NPR1-TGA2/TGA5/TGA6 regulate SARD1 (b) SARD1 regulates DLO1 expression (Figure 9). Because DLO1 gene is not only TGA1/TGA4-dependent after SA treatment but also under mock conditions, we postulate that TGA1/TGA4 act as amplifiers of this gene and NPR1-TGA2/TGA5/TGA6 together with SARD1 serve as SA-inducible activators (Figure 31). 


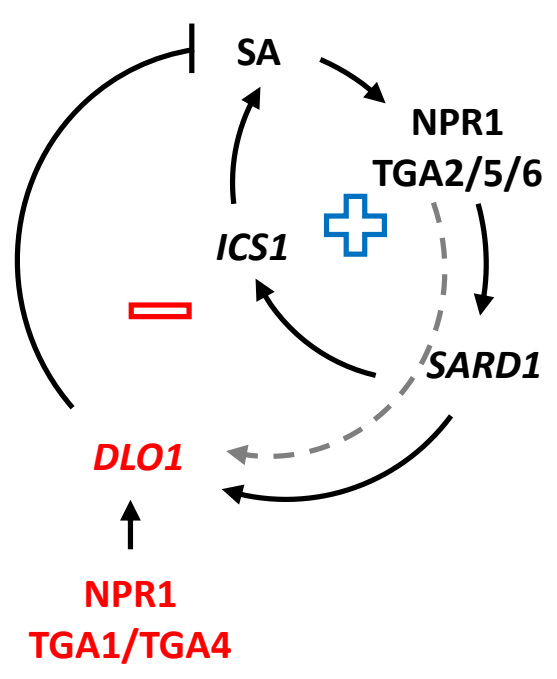

Figure 31 Negative feedback loop of SA-regulation.

SA activates NPR1, which interacts with TGA2/TGA5/TGA6 to induce transcription. NPR1 and TGA2/TGA5/TGA6 are responsible for increasing expression of SARD1, which is another transcription factor. Through regulation of SA biosynthesis gene ICS1, SARD1 is a part of a positive feedback loop which leads to SA accumulation. Another direct target of SARD1 is DLO1, which encodes for an SA catabolism enzyme. Because DLO1 degrades SA, it is a part of negative feedback loop. TGA1/TGA4 and potentially NPR1 act as amplifiers of DLO1 transcription. Not to exclude the possibility that DLO1 is a direct target of TGA2/TGA5/TGA6-NPR1 complex, a grey dashed arrow is indicated.

Blue plus marks positive feedback loop consisting of SA-NPR1-TGA2/TGA5/TGA6-SARD1-ICS1. Red minus marks negative feedback loop consisting of SA-NPR1-TGA2/TGA5/TGA6-SARD1-DLO1. Full black arrows show direct regulation and a grey dashed arrow shows a possible regulation.

\subsubsection{TGA1, TGA2 and SARD1 activate DLO1 promoter in Arabidopsis mesophyll protoplasts} and this is dependent on TGACGTCA motif in DLO1 promoter

Here we demonstrated that TGA1, TGA2 and SARD1 can activate DLO1 promoter in protoplasts and the activation was dependent on the C-box element (TGACGTCA) at DLO1 promoter (Figure 22 and Figure 23). Interestingly, the A-box element of DLO1 was not important for TGA1- and TGA2-modulated DLO1 promoter activation. This motif was previously identified as a binding element of TGA1a from tobacco using Electrophoretic Mobility Shift Assay (EMSA) (Izawa et al. 1993). I was not shown until recently that TGA1/TGA4 can bind this promoter in plants. The proteins were detected at the A-box element of ATH1 (ARABIDOPSIS THALIANA BOMEOBOX GENE1) promoter of Arabidopsis apices (Wang et al. 2019).

It could be that the binding-affinity of TGA1 and TGA4 toward different elements is due to the different interaction partners. Their role in development is connected with BOP1/BOP2 while 
their role in immunity is connected with NPR1 (Wang et al. 2019; Després et al. 2003). Interestingly, BOP1/BOP2 and NPR1 belong to the same protein family (Hepworth et al. 2005). Thus, it is possible that these interactors guide TGA1/TGA4 towards different promoter regions.

As opposed to TGA2 which preferentially binds to as1-element, which consists of two TGA binding sites separated by 12 base pairs, TGA1 can also bind to a single TGACG motif (Lam and Lam 1995). Because DLO1 promoter has only one TGA binding site, we postulated it was a direct target of TGA1. The data from protoplast assay with TGA1-VP and TGA2-VP shows that both proteins are able to activate DLO1 promoter (Figure 22). As mentioned above, the activation was dependent on TGACGTCA motif (Figure 23). One possibility is that the factors bind as heterodimers. A study from tobacco showed TGA1 homodimer or heterodimer with either TGA2.1 or TGA2.2 occupied only one TGA motif of as1-element (Niggeweg et al. 2000). The same element of DLO1 promoter could be occupied with heterodimers. However, if we postulate that TGA1/TGA4 are amplifiers of DLO1 transcription, than the promoter is either bound by TGA1 homodimer or TGA1-TGA2 heterodimer (Figure 32A and Figure 32B).

DLO1 promoter contains two SARD1 binding sites and it was activated in presence of SARD1 in protoplasts (Figure 10 and Figure 22). Thus, we think that DLO1 is a direct target of SARD1. In fact, we termed SARD1 as an SA-switchable component of DLO1 regulation. Because activation of SARD1 was TGA1/TGA4-dependent only in mock situation but not after SA treatment, TGA1/TGA4 are not responsible for induction of SARD1 after SA (Figure 5). We showed that SARD1 induction is TGA2/TGA5/TGA6- and NPR1-dependent before and after SA treatment (Figure 10). Therefore, we propose a model where SARD1 is directly regulated via TGA2/TGA5/TGA6-NPR1 complex downstream of SA (Figure 32B). Finally, SARD1 binds to DLO1 promoter and induces it (Figure 32C). An easy experiment to address the binding of SARD1 to DLO1 promoter, would be to mutate SARD1-binding site at the promoter and measure the promoter activity using Dual-Luciferase Reporter (DLR ${ }^{\mathrm{TM}}$ ) assay in protoplasts.

Although data from protoplast assay leave many questions open, we can say that TGA1, TGA2 and SARD1 activated DLO1 promoter. Moreover, activation via TGA1 and TGA2 was strongly dependent on TGACGTCA motif at the promoter. 

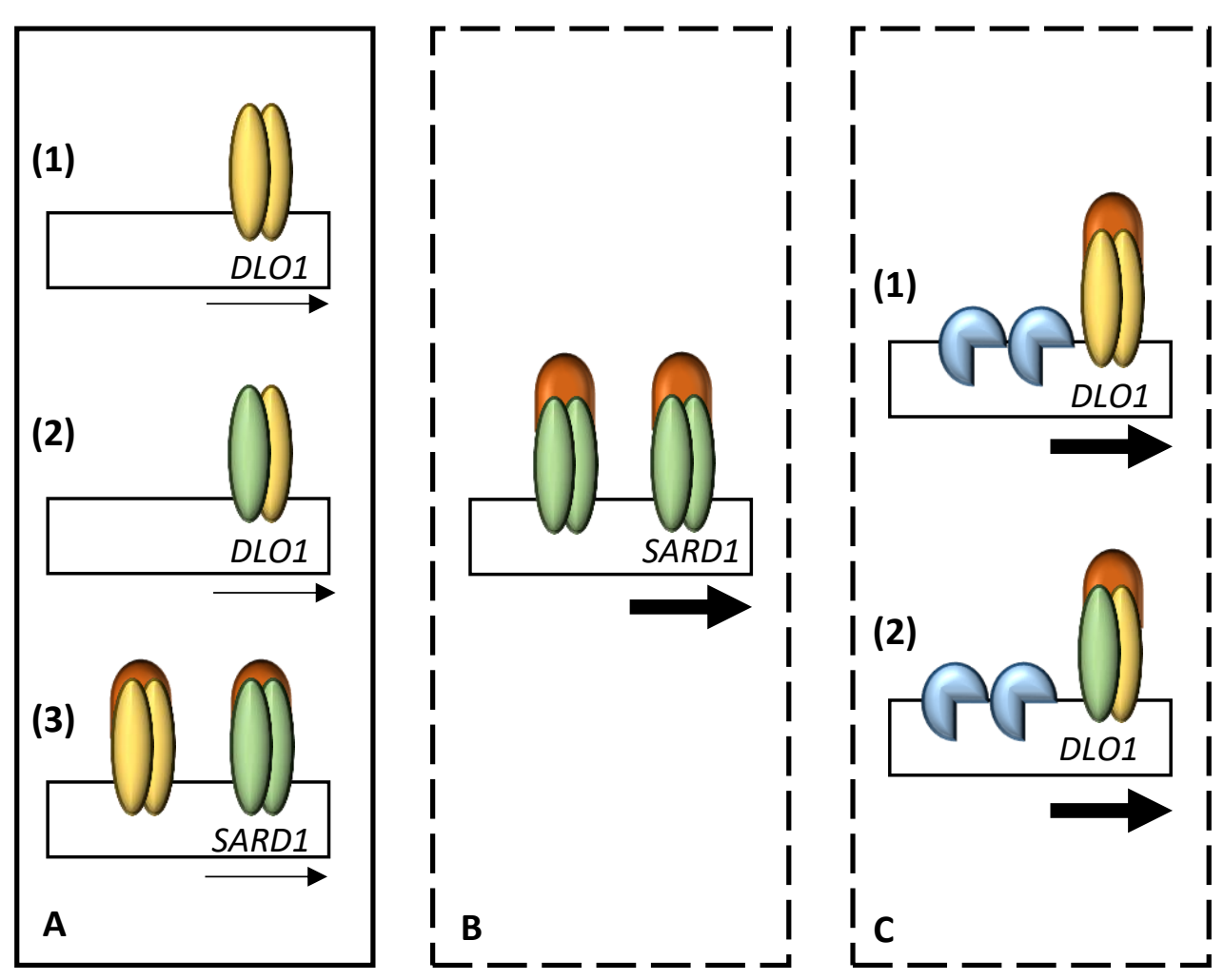

Figure 32 Proposed models for regulation of DLO1 transcription.

A Without outside stimulus, DLO1 could be regulated by either TGA1 homodimers (1) or TGA1-TGA2 heterodimers (2) binding to a single palindrome in the promoter region. On the other hand, SARD1 transcription is regulated by TGA1-NPR1 and TGA2-NPR1 (3).

B After SA treatment, first regulatory step includes induction of SARD1 via TGA2/TGA5/TGA6-NPR1.

C Downstream of SARD1 transcription, there are two possibilities of $D L O 1$ regulation. DLO1 is directly regulated by TGA1-NPR1 complex and SARD1 (1) or TGA1-TGA2-NPR1 ternary complex and SARD1 (2).

Transcriptional factors are color coded, TGA1 is shown in yellow, TGA2 in green, SARD1 in blue and transcriptional coactivator NPR1 in orange. Black box represents uninduced conditions and dashed box induced state after SA treatment. Arrows beneath the promoter indicate transcriptional activation with thickness corresponding to the activation strength. 


\subsection{Are critical cysteine residues important for TGA1/TGA4 role downstream of SA?}

\subsubsection{Critical cysteine residues are not important for induction of TGA1/TGA4-dependent genes downstream of salicylic acid}

Current model for TGA1 protein regulation via reduction of disulfide bond, glutathionylation and nitrosylation is taken as a fact although in vivo data for this model is missing (Després et al. 2003; Lindermayr et al. 2010). Our data challenges this model showing that TGA1-redox state is not important for its activity. Després and colleagues showed that already in uninduced state, that is when plants were mock-treated, approximately half of the TGA1protein pool was reduced. In our TGA1gr complementation line TGA1 protein existed only in reduced state. Thus, one would expect that at least the basal levels of TGA1/TGA4-dependent genes would be increased in this complementation line. This was not observed.

Using pharmacological treatment with SA, we showed that TGA1 redox state was not important for induction of downstream genes (Figure 12). Perhaps under our conditions, already the native TGA1 (TGA1g) was fully reduced and thus we did not observe any difference between the reduced and native form of the complementation. Another explanation for the observation could be that NPR1 protein is the limiting factor for induction of genes. If there would be unlimited amount of NPR1 in the nucleus, perhaps we would be able to see stronger or faster activation of downstream genes in TGA1gr complementation lines. Unfortunately, NPR1 does not only interact with TGA1/TGA4 but also with other TGA factors and the constitutive nuclear expression would have pleiotropic effects (Zhang et al. 1999; Després et al. 2003). Another option would be to complement tga1 tga4 mutant with the TGA1-oxidized form which is thought to be inactive (Després et al, 2003). So far, there is no described method which would prevent disulfide bridge from reduction in vivo.

\subsubsection{The critical cysteine residues are not important for induction of TGA1/TGA4- dependent genes after Pseudomonas syringae infection}

Infection with hemi-biotrophic pathogen Pseudomonas syringae leads to the accumulation of salicylic acid and nitric oxide (NO) in infected tissue (Durrant and Dong 2004; Delledonne et 
al. 1998).Moreover, NO treatment alone was also shown to induce defense genes (Palmieri et al. 2008). Because both are reported to modulate TGA1-redox state, we used infection assay to induce accumulation of both molecules and address the role of the critical cysteine residues. We demonstrated that TGA1/TGA4-marker genes were inducible by Pseudomonas syringae and that this induction was indeed TGA1/TGA4-dependent (Figure 13). Again, we could show that TGA1-redox state was not important for the activation of TGA1/TGA4dependent genes after Pseudomonas infection (Figure 14). In hand with this goes the data that TGA1 transcription was induced upon infection, indicating that activation of genes might not be due to redox regulation but rather de novo synthesis of TGA1 protein (Figure 18). However, if TGA1 would be autoregulated, there would be the chance that redox regulation would play a role.

\subsubsection{Induction of TGA1/TGA4-dependent genes after pathogen infection depends on NPR1-TGA2/TGA5/TGA6 and SARD1/CBP60g}

Upon pathogen infection, TGA1 was reported to be a transcriptional activator of SARD1 (Sun et al. 2018). This correlated with lower levels of SARD1 transcript and consequently less SA accumulation. Under our condition, SARD1 control by TGA1/TGA4 varies in different experiments (Figure 13 and Figure 27). Nevertheless, expression of two other TGA1/TGA4dependent marker genes, DLO1 and BGL2, was always lower in tga1 tga4 mutant after infection (Figure 13 and Figure 27). Thus, we agree with the proposed model that TGA1 functions upstream of SARD1 and SA, but we think there is also an additional function of TGA1 which is not described yet and this influences expression of our marker genes DLO1 and BGL2. Since the induction of marker genes was also dependent on NPR1-TGA2/TGA5/TGA6 and SA, TGA1-dependent genes could be a part of an SA amplification loop.

We propose the following model, described in the figure below (Figure 33). Pathogen infection leads to the activation of TGA1-NPR1 complex. Because basal levels of SARD1 were lower in both tga1 tga4 and npr1 mutant, we propose that they act together to activate the transcription of SARD1 (Figure 33) (Sun et al. 2018). Downstream of NPR1-TGA1, SARD1 activates ICS1 transcription. Accumulation of SA leads to a redox shift in the cell towards more reducing conditions and consequently increase of nuclear NPR1 protein levels. Interaction of NPR1 with TGA2/TGA5/TGA6 activates transcription of downstream genes. Because SARD1 
levels were lower in tga $2 \operatorname{tga} 5 \operatorname{tga} 6$ mutant than in the wild-type but not to the same extent as in tga1 tga4 mutant, there is an additional activation of SARD1 by TGA2/TGA5/TGA6-NPR1 which in favor activates ICS1 (Figure 13) (Figure 33-blue arrows). It is possible that NPR1TGA2/TGA5/TGA6 feed into the SA-activation loop on a higher level by regulation of TGA1 (Figure 33-blue arrow with a question mark). Moreover, NPR1-TGA2/TGA5/TGA6 either directly or through regulation of SARD1 activate transcription of DLO1. Activity of DLO1 leads to degradation of SA thus suppresses its accumulation (Figure 33-red loop). Because DLO1 is less expressed in tga1 tga4 mutant and TGA1 activates its promoter in protoplast, we cannot exclude there is an additional regulation of DLO1 directly through TGA1.

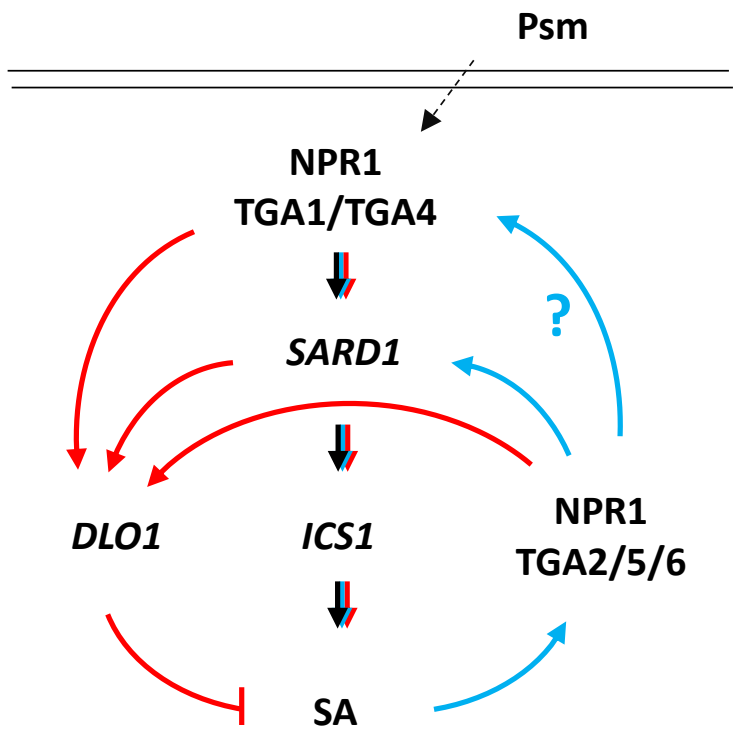

Figure 33 Proposed model for function of TGA1/TGA4 in activation of defense response after pathogen infection.

Pseudomonas syringae (Psm) infection causes changes which lead to activation of NPR1-TGA1/TGA4 complex through an unknown mechanism. NPR1-TGA1/TGA4 activate SARD1 transcription which leads to SA biosynthesis and accumulation (Sun et al, 2018). Downstream of SA biosynthesis, NPR1TGA2/TGA5/TGA6 complex activates defense genes either directly or indirectly through SARD1. Through regulation of SA-biosynthesis gene ICS1, SARD1 is a part of a positive feedback loop which leads to $S A$ accumulation, here shown in blue. It could also be that TGA1 transcript is regulated directly through NPR1-TGA2/TGA5/TGA6 and TGA1 is also a part of a positive feedback loop (blue arrow with question mark). Another direct target of SARD1 is DLO1, SA catabolism enzyme. Because DLO1 degrades $S A$, it is a part of negative feedback loop shown in red. Because TGA1, TGA2 and SARD1 activate DLO1 promoter in Arabidopsis protoplast, they could all be direct regulators of DLO1 (red arrows).

Positive feedback loop resulting in SA accumulation is shown in blue and negative feedback loop resulting in SA degradation is shown in red. Full arrows show direct regulation and dashed arrows show indirect regulation. 


\subsection{Do highly expressed glutaredoxins in tga1 tga4 mutant suppress the activity of TGA2/TGA5/TGA6?}

\subsubsection{ROXY11-15 are not repressors of TGA2/TGA5/TGA6 in tga1 tga4 mutant}

CC-type glutaredoxins, ROXYs, are known to interact with TGA transcription factors. The first described ROXY1/ROXY2 are known repressors of PAN in flower development and activators of TGA9/TGA10 in anther development (Xing et al. 2005; Murmu et al. 2010).

ROXY19 was described as negative regulator of TGA2/TGA5/TGA6 (Ndamukong et al. 2007; Zander et al. 2012; Zander et al. 2010; Huang et al. 2016). Although all ROXYs were able to interact with TGA2, only the ones with a C-terminal ALWL motif were able to repress its activity (Zander et al. 2012). This motif was responsible for interaction with transcriptional corepressor TOPLESS (Uhrig et al. 2017). All ROXYs, but ROXY6-9, possess this motif. Here we showed that all ALWL containing ROXYs are higher expressed in sid2 tga1 tga4 mutant than in the sid2 mutant regardless of SA treatment (Figure 24). Because of their known role as repressors of TGA2/TGA5/TGA6, we wanted to address if the highly expressed ROXYs were responsible for repression of TGA2/TGA5/TGA6 in tga1 tga4 mutant which was observed as lower expression of TGA1/TGA4-dependent genes. To do so, we used CRISPR-Cas9 technology to induce deletion of a cluster consisting of five ROXY genes, namely ROXY11-15.

If the hypothesis was correct, it was expected that the knock-out of the five ROXY genes in tga1 tga4 mutant would restore wild-type-like behavior. We demonstrated that the knockout of ROXY11-15 genes in tga1 tga4 mutant did not restore wild-type behavior. The mutant had lower induction of DLO1 than wild-type, just like tga1 tga4 mutant (Figure 26). Similarly, after pathogen infection, induction of marker genes was significantly lower than in the wildtype but not different than in the tga1 tga4 mutant (Figure 27). Moreover, the susceptibility of roxy11-15 tga1 tga4 to biotrophic pathogen Pseudomonas syringae was not significantly different than the susceptibility of tga1 tga4 mutant (Figure 28). This all led to conclusion that ROXY11-15 are not responsible for repression of TGA2/TGA5/TGA6 in tga1 tga4 mutant. However, we cannot exclude that the other highly expressed ROXYs redundantly repress TGA2/TGA5/TGA6 proteins in tga1 tga4 mutant. 


\section{Discussion}

Interestingly, SA and pathogen induction of marker genes in roxy11-15 mutant was lower than in the wild-type plants (Figure 26 and Figure 27). Since the induction was still stronger than in tga1 tga4 mutant, it could be that ROXY11-15 and TGA1/TGA4 work in the same cascade, where ROXY11-15 are placed upstream. Because this observation varied between experiments, we cannot draw any conclusion from this data.

\subsection{Because TGA1 is repressed by ectopically expressed ROXY9, does ROXY9 loss-of-function have a reverse effect?}

\subsubsection{ROXY9-mediated repression of TGA1/TGA4 is not released after SA treatment}

ROXY9 belongs to those four ROXYs (ROXY6, ROXY7, ROXY8 and ROXY9) that do not contain an ALWL motif. When over-expressed, ROXY9 represses TGA1/TGA4-regulated hyponastic growth (Li et al. 2019). We show here that it also repressed TGA1/TGA4-dependent activation of SA-inducible genes (Figure 29). One of the aims of the project was to construct a roxy6 roxy7 roxy8 roxy9 quadruple mutant in order to obtain loss-of-function evidence for the repressing function of ROXYs. We used CRIPRS-Cas9 technology to obtain single mutants and crossing to obtain higher order mutants.

Although we expected overall release of repression of TGA1/TGA4 in roxy6789 mutant, this was not observed after SA treatment. On contrary, roxy6789 mutant displayed lower levels of marker genes before and after SA treatment (Figure 30). Further experiments have to be performed to verify the preliminary data. 


\section{Summary}

TGA1 and TGA4 belong to a family of basic leucine zipper transcription factors. They are important activators of salicylic acid (SA) biosynthesis upon pathogen infection (Sun et al. 2018). Moreover, they interact with a master regulator of SA signaling NPR1 in a redox-dependent manner (Després et al. 2003; Lindermayr et al. 2010). However, the role of TGA1 and TGA4 downstream of SA signaling was not known. Here, we report on a group of TGA1/TGA4-dependent genes which were inducible by SA and pathogen infection. The identified genes belonged to the known signaling pathway downstream of SA consisting of NPR1, TGA2/TGA5/TGA6 and SARD1/CBP60g. Because the marker genes were both TGA1/TGA4- and NPR1-dependent, they were used to investigate the redox regulation of TGA1. The tga1 tga 4 mutant was complemented either with a wild-type TGA1 protein or a TGA1 protein mutated in such way to mimic the reduced, active form of the protein. We showed that the two types of complementation lines restore wild-type-like behavior after SA treatment and pathogen infection. Thus, we concluded that the redox state of the four critical cysteine residues is not important for the function of TGA1 downstream of SA signaling.

In addition to the direct redox-regulation of the cysteine residues, TGA1 and TGA4 proteins are negatively regulated by CC-type glutaredoxins ROXYs (Li et al. 2019). To investigate regulation of TGA factors by glutaredoxins, we generated mutants of two groups of ROXY proteins. The two groups repress either clade I TGA or clade II TGA factors depending on the presence of the C terminal ALWL motif (Zander et al. 2012; Uhrig et al. 2017). The members of the ALWL group, which repress clade II TGA transcription factors, were highly expressed in tga1 tga4 mutant. We showed here that the mutation in five ALWL ROXY genes (ROXY11-15) did not restore wild-type like behavior in tga1 tga4 mutant. However, it is plausible that the twelve remaining ROXYs repress clade II TGA factors in roxy1115 tga1 tga4 mutant. Furthermore, we generated a mutant of the four ROXY genes lacking the ALWL motif (ROXY6-9), which repress TGA1 and TGA4 function when overexpressed. Here, we showed that the mutation in ROXY6-9 did not lead to the hyperactivity of clade I TGA factors after SA spraying and pathogen infection. However, it is possible that the repression is lifted under different conditions. 


\section{Publication Bibliography}

Alfano, James R.; Collmer, Alan (2004): Type III secretion system effector proteins: double agents in bacterial disease and plant defense. In Annual review of phytopathology 42, pp. 385-414. DOI: 10.1146/annurev.phyto.42.040103.110731.

Alvarez, José M.; Riveras, Eleodoro; Vidal, Elena A.; Gras, Diana E.; Contreras-López, Orlando; Tamayo, Karem P. et al. (2014): Systems approach identifies TGA1 and TGA4 transcription factors as important regulatory components of the nitrate response of Arabidopsis thaliana roots. In The Plant journal : for cell and molecular biology 80 (1), pp. 1-13. DOI: 10.1111/tpj.12618.

Ashburner, Michael; Ball, Catherine A.; Blake, Judith A.; Botstein, David; Butler, Heather; Cherry, J. Michael et al. (2000): Gene Ontology: tool for the unification of biology. In Nature genetics 25 (1), pp. 25-29. DOI: 10.1038/75556.

Beckers, G. J. M.; Spoel, S. H. (2006): Fine-Tuning Plant Defence Signalling: Salicylate versus Jasmonate. In Plant biology (Stuttgart, Germany) 8 (1), pp. 1-10. DOI: 10.1055/s-2005-872705.

Berendzen, Kenneth W.; Weiste, Christoph; Wanke, Dierk; Kilian, Joachim; Harter, Klaus, Dröge-Laser, Wolfgang (2012): Bioinformatic cis-element analyses performed in Arabidopsis and rice disclose bZIPand MYB-related binding sites as potential AuxRE-coupling elements in auxin-mediated transcription. In BMC plant biology 12 (125).

Bigeard, Jean; Colcombet, Jean; Hirt, Heribert (2015): Signaling mechanisms in pattern-triggered immunity (PTI). In Molecular plant 8 (4), pp. 521-539. DOI: 10.1016/j.molp.2014.12.022.

Cao, Hui; Bowling, Scott A.; Gordon, Susan A.; Dong, Xinnian (1994): Characterization of an Arabidopsis mutant that is nonresponsive to inducers of systemic acquired resistance. In The Plant cell 6, pp. 15831592.

Chen, Yun-Chu; Holmes, Eric C.; Rajniak, Jakub; Kim, Jung-Gun; Tang, Sandy; Fischer, Curt R. et al. (2018): N-hydroxy-pipecolic acid is a mobile metabolite that induces systemic disease resistance in Arabidopsis. In Proceedings of the National Academy of Sciences of the United States of America 115 (21), E4920-E4929. DOI: 10.1073/pnas.1805291115.

Choi, Jaemyung; Huh, Sung Un; Kojima, Mikiko; Sakakibara, Hitoshi; Paek, Kyung-Hee; Hwang, Ildoo (2010): The cytokinin-activated transcription factor ARR2 promotes plant immunity via TGA3/NPR1dependent salicylic acid signaling in Arabidopsis. In Developmental cell 19 (2), pp. 284-295. DOI: 10.1016/j.devcel.2010.07.011.

Chomczynski, P. (1993): A reagent for the single-step simultaneous isolation of RNA, DNA and proteins from cell and tissue samples. In BioTechniques 15 (3), 532-4, 536-7.

Chuang, Chiou-Fen; Running, Mark P.; Williams, Robert W.; Meyerowitz, Elliot M. (1999): The PERIANTHIA gene encodes a bZIP protein involved in the determination of floral organ number in Arabidopsis thaliana. In Genes \& Development 13, pp. 334-344. 
Clough, S. J.; Bent, A. F. (1998): Floral dip: a simplified method for Agrobacterium-mediated transformation of Arabidopsis thaliana. In The Plant journal : for cell and molecular biology 16 (6), pp. 735-743. DOI: 10.1046/j.1365-313x.1998.00343.x.

Couturier, Jérémy; Jacquot, Jean-Pierre; Rouhier, Nicolas (2009): Evolution and diversity of glutaredoxins in photosynthetic organisms. In Cellular and molecular life sciences : CMLS 66 (15), pp. 2539-2557. DOI: 10.1007/s00018-009-0054-y.

Delaney, T. P.; Friedrich, L.; Ryals, J. A. (1995): Arabidopsis signal transduction mutant defective in chemically and biologically induced disease resistance. In Proceedings of the National Academy of Sciences of the United States of America 92, pp. 6602-6606.

Delledonne, Massimo; Xia, Yiji; Dixon, Richard A.; Lamb, Chris (1998): Nitric oxide functions as a signal in plant disease resistance. In Nature 394, pp. 585-588.

Dempsey, D'Maris Amick; Vlot, A. Corina; Wildermuth, Mary C.; Klessig, Daniel F. (2011): Salicylic Acid biosynthesis and metabolism. In The arabidopsis book 9, e0156. DOI: 10.1199/tab.0156.

Després, Charles; Chubak, Catherine; Rochon, Amanda; Clark, Rena; Bethune, Terry; Desveaux, Darrell; Fobert, Pierre R. (2003): The Arabidopsis NPR1 disease resistance protein is a novel cofactor that confers redox regulation of DNA binding activity to the basic domain/leucine zipper transcription factor TGA1. In The Plant cell 15 (9), pp. 2181-2191. DOI: 10.1105/tpc.012849.

Després, Charles; DeLong, Catherine; Glaze, Sarah; Liu, Enwu; Fobert, Pierre R. (2000): The Arabidopsis NPR1/NIM1 protein enhances the DNA binding activity of a subgroup of the TGA family of bZIP transcription factors. In The Plant cell 12, pp. 279-290.

Ding, Pingtao; Rekhter, Dmitrij; Ding, Yuli; Feussner, Kirstin; Busta, Lucas; Haroth, Sven et al. (2016): Characterization of a Pipecolic Acid Biosynthesis Pathway Required for Systemic Acquired Resistance. In The Plant cell 28 (10), pp. 2603-2615. DOI: 10.1105/tpc.16.00486.

Ding, Yuli; Sun, Tongjun; Ao, Kevin; Peng, Yujun; Zhang, Yaxi; Li, Xin; Zhang, Yuelin (2018): Opposite Roles of Salicylic Acid Receptors NPR1 and NPR3/NPR4 in Transcriptional Regulation of Plant Immunity. In Cell 173 (6), 1454-1467.e15. DOI: 10.1016/j.cell.2018.03.044.

Durrant, W. E.; Dong, X. (2004): Systemic acquired resistance. In Annual review of phytopathology 42, pp. 185-209. DOI: 10.1146/annurev.phyto.42.040803.140421.

Engler, Carola; Gruetzner, Ramona; Kandzia, Romy; Marillonnet, Sylvestre (2009): Golden gate shuffling: a one-pot DNA shuffling method based on type lls restriction enzymes. In PloS one 4 (5), e5553. DOI: 10.1371/journal.pone.0005553.

Feechan, Angela; Kwon, Eunjung; Yun, Byung-Wook; Wang, Yiqin; Pallas, Jacqueline A.; Loake, Gary J. (2005): A central role for S-nitrosothiols in plant disease resistance. In PNAS 102 (22), pp. 8054-8059. DOI: $10.1073 /$ pnas.0501456102.

Flor, Harold Henry (1971): Current Status of the Gene-For-Gene Concept. In Annual review of phytopathology 9, pp. 275-296.

Fu, Zheng Qing; Yan, Shunping; Saleh, Abdelaty; Wang, Wei; Ruble, James; Oka, Nodoka et al. (2012): NPR3 and NPR4 are receptors for the immune signal salicylic acid in plants. In Nature 486 (7402), pp. 228-232. DOI: 10.1038/nature11162. 
Glazebrook, Jane (2005): Contrasting mechanisms of defense against biotrophic and necrotrophic pathogens. In Annual review of phytopathology 43, pp. 205-227. DOI: 10.1146/annurev.phyto.43.040204.135923.

Gutsche, Nora; Thurow, Corinna; Zachgo, Sabine; Gatz, Christiane (2015): Plant-specific CC-type glutaredoxins: functions in developmental processes and stress responses. In Biological chemistry 396 (5), pp. 495-509. DOI: 10.1515/hsz-2014-0300.

Hanahan, Douglas (1983): Studies on transformation of Escherichia coli with plasmids. In Journal of Molecular Biology 166 (4), pp. 557-580. DOI: 10.1016/S0022-2836(83)80284-8.

Hartmann Michael; Kim Denis; Bernsdorff Friederike; Ajami-Rashidi Ziba; Scholten Nicola; Schreiber Stefan; Zeier Tatyana, Schuck Stefan; Reichel-Deland Vanessa; Zeier Jürgen. Biochemical Principles and Functional Aspects of Pipecolic Acid Biosynthesis in Plant Immunity. Plant Physiol. 2017;174(1):124153. doi:10.1104/pp.17.00222.

Hartmann, Michael; Zeier, Tatyana; Bernsdorff, Friederike; Reichel-Deland, Vanessa; Kim, Denis; Hohmann, Michele et al. (2018): Flavin Monooxygenase-Generated N-Hydroxypipecolic Acid Is a Critical Element of Plant Systemic Immunity. In Cell 173 (2), 456-469.e16. DOI: 10.1016/j.cell.2018.02.049.

Hauck, Paula; Thilmony, Roger; He, Sheng Yang (2003): A Pseudomonas syringae type III effector suppresses cell wall-based extracellular defense in susceptible Arabidopsis plants. In Proceedings of the National Academy of Sciences of the United States of America 100 (14), pp. 8577-8582. DOI: 10.1073/pnas.1431173100.

Hepworth, Shelley R.; Zhang, Yuelin; McKim, Sarah; Li, Xin; Haughn, George W. (2005): BLADE-ONPETIOLE-dependent signaling controls leaf and floral patterning in Arabidopsis. In The Plant cell 17 (5), pp. 1434-1448. DOI: 10.1105/tpc.104.030536.

Houston, Kelly; Tucker, Matthew R.; Chowdhury, Jamil; Shirley, Neil; Little, Alan (2016): The Plant Cell Wall: A Complex and Dynamic Structure As Revealed by the Responses of Genes under Stress Conditions. In Frontiers in plant science 7, p. 984. DOI: 10.3389/fpls.2016.00984.

Huang, Li-Jun; Li, Ning; Thurow, Corinna; Wirtz, Markus; Hell, Rüdiger; Gatz, Christiane (2016): Ectopically expressed glutaredoxin ROXY19 negatively regulates the detoxification pathway in Arabidopsis thaliana. In BMC plant biology 16 (1), p. 200. DOI: 10.1186/s12870-016-0886-1.

Hulsen, Tim; Vlieg, Jacob de; Alkema, Wynand (2008): BioVenn -a web application for the comparison and visualization of biological lists using area-proportional Venn diagrams. In BMC genomics 9, p. 488. DOI: 10.1186/1471-2164-9-488.

Izawa, Takeshi; Foster, Randy; Chua, Nam-Hai (1993): Plant bZIP Protein DNA Binding Specificity. In Journal of Molecular Biology 230, pp. 1131-1144.

Jakoby, Marc; Weisshaara, Bernd; Dröge-Laser, Wolfgang; Vicente-Carbajosac, Jesus; Tiedemannd, Jens; Kroj, Thomas; Parcy, François (2002): bZIP transcription factors in Arabidopsis. In Trends in Plant Science 7 (3), 106-111.

Jones, Jonathan D. G.; Dangl, Jeffery L. (2006): The plant immune system. In Nature 444 (7117), pp. 323-329. DOI: 10.1038/nature05286. 
Jyoti Shah; Frank Tsui; and Daniel F. Klessig (1997): Characterization of a Salicylic Acid-Insensitive Mutant (sai1) of Arabidopsis thaliana, Identified in a Selective Screen Utilizing the SA-Inducible Expression of the tms2 Gene. In MPMI 10, pp. 69-78.

Katagiri, Fumiaki; Lam, Eric; Chua, Nam-Hai (1989): Two tobacco DNA-binding proteins with homology to the nuclear factor CREB. In Nature 340, 727-730.

Katagiri, Fumiaki; Thilmony, Roger; He, Sheng Yang (2002): The Arabidopsis thaliana-pseudomonas syringae interaction. In The arabidopsis book 1, e0039. DOI: 10.1199/tab.0039.

Katzen, Federico (2007): Gateway $\left({ }^{\oplus}\right)$ recombinational cloning: a biological operating system. In Expert opinion on drug discovery 2 (4), pp. 571-589. DOI: 10.1517/17460441.2.4.571.

Kesarwani, Meenu; Yoo, Jungmin; Dong, Xinnian (2007): Genetic interactions of TGA transcription factors in the regulation of pathogenesis-related genes and disease resistance in Arabidopsis. In Plant physiology 144 (1), pp. 336-346. DOI: 10.1104/pp.106.095299.

Kim, Yongsin; Gilmour, Sarah J.; Chao, Lumen; Park, Sungchung; Thomashow, Michael F. (2019) Arabidopsis CAMTA transcription factors regulate pipecolic acid biosynthesis and priming for immunity genes. Molecular Plant 13: 157-168.

Kinkema, Mark; Fan, Weihua; Dong, Xinnian (2000): Nuclear Localization of NPR1 Is Required for Activation of PR Gene Expression. In The Plant cell 12, pp. 2339-3250.

Koncz, Csaba; Schell, Jeff (1986): The promoter of TL-DNA gene 5 controls the tissue-specific expression of chimaeric genes carried by a novel type of Agrobacterium binary vector. In Molecular and General Genetics 204, pp. 383-396.

Lam, E.; Lam, Y. K. (1995): Binding site requirements and differential representation of TGF factors in nuclear ASF-1 activity. In Nucleic acids research 23 (18), pp. 3778-3785.

Lee, Hyo-Jun; Park, Young-Joon; Seo, Pil Joon; Kim, Ju-Heon; Sim, Hee-Jung; Kim, Sang-Gyu; Park, Chung-Mo (2015): Systemic Immunity Requires SnRK2.8-Mediated Nuclear Import of NPR1 in Arabidopsis. In The Plant cell 27 (12), pp. 3425-3438. DOI: 10.1105/tpc.15.00371.

Li, Ning; Muthreich, Martin; Huang, Li-Jun; Thurow, Corinna; Sun, Tongjun; Zhang, Yuelin; Gatz, Christiane (2019): TGACG-BINDING FACTORs (TGAs) and TGA-interacting CC-type glutaredoxins modulate hyponastic growth in Arabidopsis thaliana. In The New phytologist 221 (4), pp. 1906-1918. DOI: $10.1111 /$ nph.15496.

Li, Shutian; Lauri, Andrea; Ziemann, Mark; Busch, Andrea; Bhave, Mrinal; Zachgo, Sabine (2009): Nuclear activity of ROXY1, a glutaredoxin interacting with TGA factors, is required for petal development in Arabidopsis thaliana. In The Plant cell 21 (2), pp.429-441. DOI: 10.1105/tpc.108.064477.

Lin, Nai-Chun; Martin, Gregory B. (2005): An avrPto/avrPtoB mutant of Pseudomonas syringae pv. tomato DC3000 does not elicit Pto-mediated resistance and is less virulent on tomato. In Molecular plant-microbe interactions : MPMI 18 (1), pp. 43-51. DOI: 10.1094/MPMI-18-0043.

Lindermayr, Christian; Sell, Simone; Müller, Bernd; Leister, Dario; Durner, Jörg (2010): Redox regulation of the NPR1-TGA1 system of Arabidopsis thaliana by nitric oxide. In The Plant cell 22 (8), pp. 28942907. DOI: 10.1105/tpc.109.066464. 
Livak, K. J.; Schmittgen, T. D. (2001): Analysis of relative gene expression data using real-time quantitative PCR and the 2(-Delta Delta C(T)) Method. In Methods (San Diego, Calif.) 25 (4), pp. 402408. DOI: $10.1006 /$ meth.2001.1262.

Lohse, Marc; Bolger, Anthony M.; Nagel, Axel; Fernie, Alisdair R.; Lunn, John E.; Stitt, Mark; Usadel, Björn (2012): RobiNA: a user-friendly, integrated software solution for RNA-Seq-based transcriptomics. In Nucleic acids research 40 (Web Server issue), W622-7. DOI: 10.1093/nar/gks540.

Martin, Jennifer L. (1995): Thioredoxin -a fold for all reasons. In Structure 3, pp. 245-250.

Mattanovich, D.; Rüker, F.; Machado, A. C.; Laimer, M.; Regner, F.; Steinkellner, H. et al. (1989): Efficient transformation of Agrobacterium spp. by electroporation. In Nucleic acids research 17 (16), p. 6747. DOI: $10.1093 /$ nar/17.16.6747.

Mean, Richard James; Pierides, Alkis; Deltas, C. Constantinou; Koptides, Michael (2004): Modification of the enzyme mismatch cleavage method using $\mathrm{T7}$ endonuclease I and silver staining. In BioTechniques 36 (5), pp. 758-760. DOI: 10.2144/04365BM01.

Meyer, Yves; Siala, Wafi; Bashandy, Talaat; Riondet, Christophe; Vignols, Florence; Reichheld, Jean Philippe (2008): Glutaredoxins and thioredoxins in plants. In Biochimica et biophysica acta 1783 (4), pp. 589-600. DOI: 10.1016/j.bbamcr.2007.10.017.

Mi, Huaiyu; Muruganujan, Anushya; Ebert, Dustin; Huang, Xiaosong; Thomas, Paul D. (2019): PANTHER version 14: more genomes, a new PANTHER GO-slim and improvements in enrichment analysis tools. In Nucleic acids research 47 (D1), D419-D426. DOI: 10.1093/nar/gky1038.

Mishina, Tatiana E.; Zeier, Jürgen (2006): The Arabidopsis flavin-dependent monooxygenase FMO1 is an essential component of biologically induced systemic acquired resistance. In Plant physiology 141 (4), pp. 1666-1675. DOI: 10.1104/pp.106.081257.

Mou, Zhonglin; Fan, Weihua; Dong, Xinnian (2003): Inducers of Plant Systemic Acquired Resistance Regulate NPR1 Function through Redox Changes. In Cell 113 (7), pp. 935-944. DOI: 10.1016/S00928674(03)00429-X.

Murmu, Jhadeswar; Bush, Michael J.; DeLong, Catherine; Li, Shutian; Xu, Mingli; Khan, Madiha et al. (2010): Arabidopsis basic leucine-zipper transcription factors TGA9 and TGA10 interact with floral glutaredoxins ROXY1 and ROXY2 and are redundantly required for anther development. In Plant physiology 154 (3), pp. 1492-1504. DOI: 10.1104/pp.110.159111.

Návarová, Hana; Bernsdorff, Friederike; Döring, Anne-Christin; Zeier, Jürgen (2012): Pipecolic acid, an endogenous mediator of defense amplification and priming, is a critical regulator of inducible plant immunity. In The Plant cell 24 (12), pp. 5123-5141. DOI: 10.1105/tpc.112.103564.

Nawrath, Christiane; Heck, Silvia; Parinthawong, Nonglak; Métraux, Jean-Pierre (2002): EDS5, an essential component of salicylic acid-dependent signaling for disease resistance in Arabidopsis, is a member of the MATE transporter family. In The Plant cell 14 (1), pp. 275-286. DOI: 10.1105/tpc.010376.

Ndamukong, Ivan; Abdallat, Ayed Al; Thurow, Corinna; Fode, Benjamin; Zander, Mark; Weigel, Ralf; Gatz, Christiane (2007): SA-inducible Arabidopsis glutaredoxin interacts with TGA factors and 
suppresses JA-responsive PDF1.2 transcription. In The Plant journal : for cell and molecular biology 50 (1), pp. 128-139. DOI: 10.1111/j.1365-313X.2007.03039.x.

Niggeweg, Ricarda; Thurow, Corinna; Weigel, Ralf; Pfitzner, Ursula; Gatz, Christiane (2000): Tobacco TGA factors differ with respect to interaction with NPR1, activation potential and DNA-binding properties. In Plant Molecular Biology 42, pp. 775-788.

Palmieri, M. Cristina; Sell, Simone; Huang, Xi; Scherf, Matthias; Werner, Thomas; Durner, Jörg; Lindermayr, Christian (2008): Nitric oxide-responsive genes and promoters in Arabidopsis thaliana: a bioinformatics approach. In Journal of experimental botany 59 (2), pp. 177-186. DOI: 10.1093/jxb/erm345.

Pauwels, Laurens; Barbero, Gemma Fernández; Geerinck, Jan; Tilleman, Sofie; Grunewald, Wim; Pérez, Amparo Cuéllar et al. (2010): NINJA connects the co-repressor TOPLESS to jasmonate signalling. In Nature 464 (7289), pp. 788-791. DOI: 10.1038/nature08854.

Rekhter, Dmitrij; Lüdke, Daniel; Ding, Yuli; Feussner, Kirstin; Zienkiewicz, Krzysztof; Lipka, Volker et al. (2019): Isochorismate-derived biosynthesis of the plant stress hormone salicylic acid. In Science (New York, N.Y.) 365 (6452), pp. 498-502. DOI: 10.1126/science.aaw1720.

Rekhter, Dmitrij; Mohnike, Lennart; Feussner, Kirstin; Zienkiewicz, Krzysztof; Zahng uelin et al. (2019): Enhanced Disease Susceptibility 5 (EDS5) is required for $\mathrm{N}$-hydroxy pipecolic acid formation bioRxiv 630723; doi: https://doi.org/10.1101/630723.

Rochon, Amanda; Boyle, Patrick; Wignes, Tracy; Fobert, Pierre R.; Després, Charles (2006): The coactivator function of Arabidopsis NPR1 requires the core of its BTB/POZ domain and the oxidation of C-terminal cysteines. In The Plant cell 18 (12), pp.3670-3685. DOI: 10.1105/tpc.106.046953.

Seyfferth C, Tsuda K. Salicylic acid signal transduction: the initiation of biosynthesis, perception and transcriptional reprogramming. Front Plant Sci. 2014 Dec 9;5:697. doi: 10.3389/fpls.2014.00697. PMID: 25538725; PMCID: PMC4260477.

Shearer, Heather L.; Cheng, Yu Ti; Wang, Lipu; Liu, Jinman; Boyle, Patrick; Després, Charles et al. (2012): Arabidopsis clade I TGA transcription factors regulate plant defenses in an NPR1independent fashion. In Molecular plant-microbe interactions : MPMI 25 (11), pp. 1459-1468. DOI: 10.1094/MPMI-09-11-0256.

Shearer, Heather L.; Wang, Lipu; DeLong, Catherine; Després, Charles; Fobert, Pierre R. (2009): NPR1 enhances the DNA binding activity of the Arabidopsis bZIP transcription factor TGA7This paper is one of a selection of papers published in a Special Issue from the National Research Council of Canada Plant Biotechnology Institute. In Botany 87 (6), pp. 561-570. DOI: 10.1139/B08-143.

Song, Jong Tae; Lu, Hua; McDowell, John M.; Greenberg, Jean T. (2004): A key role for ALD1 in activation of local and systemic defenses in Arabidopsis. In The Plant journal : for cell and molecular biology 40 (2), pp. 200-212. DOI: 10.1111/j.1365-313X.2004.02200.x.

Spoel, Steven H.; Mou, Zhonglin; Tada, Yasuomi; Spivey, Natalie W.; Genschik, Pascal; Dong, Xinnian (2009): Proteasome-mediated turnover of the transcription coactivator NPR1 plays dual roles in regulating plant immunity. In Cell 137 (5), pp. 860-872. DOI: 10.1016/j.cell.2009.03.038. 
Ströher, Elke; Millar, A. Harvey (2012): The biological roles of glutaredoxins. In The Biochemical journal 446 (3), pp. 333-348. DOI: 10.1042/BJ20112131.

Sun, Tongjun; Busta, Lucas; Zhang, Qian; Ding, Pingtao; Jetter, Reinhard; Zhang, Yuelin (2018): TGACGBINDING FACTOR 1 (TGA1) and TGA4 regulate salicylic acid and pipecolic acid biosynthesis by modulating the expression of SYSTEMIC ACQUIRED RESISTANCE DEFICIENT 1 (SARD1) and CALMODULIN-BINDING PROTEIN 60g (CBP60g). In The New phytologist 217 (1), pp. 344-354. DOI: 10.1111/nph.14780.

Sun Tongjun; Huang Jianhua; Xu Yan; Verma Vani; Jing Beibei; Sun Yulin; Ruiz Orduna Alberto; Tian Hainan; Huang Xingchuan, Xia Shitou; Schafer Laurel; Jetter Reinhard; Zhang Yuelin; Li Xin (2020) Redundant CAMTA Transcription Factors Negatively Regulate the Biosynthesis of Salicylic Acid and NHydroxypipecolic Acid by Modulating the Expression of SARD1 and CBP60g. Mol Plant. 2020;13(1):144156. doi:10.1016/j.molp.2019.10.016

Sun, Tongjun; Zhang, Yaxi; Li, Yan; Zhang, Qian; Ding, Yuli; Zhang, Yuelin (2015): ChIP-seq reveals broad roles of SARD1 and CBP60g in regulating plant immunity. In Nature communications 6, p. 10159. DOI: 10.1038/ncomms10159.

Tada, Yasuomi; Spoel, Steven H.; Pajerowska-Mukhtar, Karolina; Mou, Zhonglin; Song, Junqi; Wang, Chun et al. (2008): Plant immunity requires conformational changes corrected of NPR1 via Snitrosylation and thioredoxins. In Science (New York, N.Y.) 321 (5891), pp.952-956. DOI: 10.1126/science.1156970.

The Gene Ontology Resource: 20 years and still GOing strong (2019). In Nucleic acids research 47 (D1), D330-D338.

Uhrig, Joachim F.; Huang, Li-Jun; Barghahn, Sina; Willmer, Moritz; Thurow, Corinna; Gatz, Christiane (2017): CC-type glutaredoxins recruit the transcriptional co-repressor TOPLESS to TGA-dependent target promoters in Arabidopsis thaliana. In Biochimica et biophysica acta. Gene regulatory mechanisms 1860 (2), pp. 218-226. DOI: 10.1016/j.bbagrm.2016.11.001.

van Wersch, Rowan; Li, Xin; Zhang, Yuelin (2016): Mighty Dwarfs: Arabidopsis Autoimmune Mutants and Their Usages in Genetic Dissection of Plant Immunity. In Frontiers in plant science 7, p. 1717. DOI: 10.3389/fpls.2016.01717.

Vernooij, Bernard; Friedrich, Leslie; Morse, Alison; Reist, Roland; Kolditz-Jawhar, Rachida; Ward, Eric et al. (1994): Salicylic Acid Is Not the Translocated Signal Responsible for Inducing Systemic Acquired Resistance but Is Required in Signal Transduction. In The Plant cell 6 (959-965).

Wang, Lin; Tsuda, Kenichi; Sato, Masanao; Cohen, Jerry D.; Katagiri, Fumiaki; Glazebrook, Jane (2009): Arabidopsis CaM binding protein CBP60g contributes to MAMP-induced SA accumulation and is involved in disease resistance against Pseudomonas syringae. In PLoS pathogens 5 (2), e1000301. DOI: 10.1371/journal.ppat.1000301.

Wang, Lin; Tsuda, Kenichi; Truman, William; Sato, Masanao; Le Nguyen, V.; Katagiri, Fumiaki; Glazebrook, Jane (2011): CBP60g and SARD1 play partially redundant critical roles in salicylic acid signaling. In The Plant journal : for cell and molecular biology 67 (6), pp. 1029-1041. DOI: 10.1111/j.1365-313X.2011.04655.x. 
Wang, Ying; Salasini, Brenda C.; Khan, Madiha; Devi, Bhaswati; Bush, Michael; Subramaniam, Rajagopal; Hepworth, Shelley R. (2019): Clade I TGACG-Motif Binding Basic Leucine Zipper Transcription Factors Mediate BLADE-ON-PETIOLE-Dependent Regulation of Development. In Plant physiology 180 (2), pp. 937-951. DOI: 10.1104/pp.18.00805.

Weber, L.; Meigel, W. N.; Rauterberg, J. (1977): SDS-polyacrylamide gel electrophoretic determination of type I and type III collagen in small skin samples. In Archives for dermatological research = Archiv fur dermatologische Forschung 258 (3), pp. 251-257.

Whalen, M. C.; Innes, R. W.; Bent, A. F.; Staskawicz, B. J. (1991): Identification of Pseudomonas syringae pathogens of Arabidopsis and a bacterial locus determining avirulence on both Arabidopsis and soybean. In The Plant cell 3 (1), pp. 49-59.

Wildermuth, Mary C.; Dewdney, Julia; Wu, Gang; Ausubel, Frederick M. (2001): Isochorismate synthase is required to synthesize salicylic acid for plant defence. In Nature 414, 562-566.

Wu, Yue; Di Zhang; Chu, Jee Yan; Boyle, Patrick; Wang, Yong; Brindle, lan D. et al. (2012): The Arabidopsis NPR1 protein is a receptor for the plant defense hormone salicylic acid. In Cell reports 1 (6), pp. 639-647. DOI: 10.1016/j.celrep.2012.05.008.

Xing, Shuping; Rosso, Mario G.; Zachgo, Sabine (2005): ROXY1, a member of the plant glutaredoxin family, is required for petal development in Arabidopsis thaliana. In Development (Cambridge, England) 132 (7), pp. 1555-1565. DOI: 10.1242/dev.01725.

Xing, Shuping; Zachgo, Sabine (2008): ROXY1 and ROXY2, two Arabidopsis glutaredoxin genes, are required for anther development. In The Plant journal : for cell and molecular biology 53 (5), pp. 790801. DOI: 10.1111/j.1365-313X.2007.03375.x.

Yeats, Trevor H.; Rose, Jocelyn K. C. (2013): The formation and function of plant cuticles. In Plant physiology 163 (1), pp. 5-20. DOI: 10.1104/pp.113.222737.

Yoo, Sang-Dong; Cho, Young-Hee; Sheen, Jen (2007): Arabidopsis mesophyll protoplasts: a versatile cell system for transient gene expression analysis. In Nature protocols 2 (7), pp. 1565-1572. DOI: 10.1038/nprot.2007.199.

Zander, Mark; Chen, Shuxia; Imkampe, Julia; Thurow, Corinna; Gatz, Christiane (2012): Repression of the Arabidopsis thaliana jasmonic acid/ethylene-induced defense pathway by TGA-interacting glutaredoxins depends on their C-terminal ALWL motif. In Molecular plant 5 (4), pp. 831-840. DOI: $10.1093 / \mathrm{mp} / \mathrm{ssr} 113$.

Zander, Mark; La Camera, Sylvain; Lamotte, Olivier; Métraux, Jean-Pierre; Gatz, Christiane (2010): Arabidopsis thaliana class-II TGA transcription factors are essential activators of jasmonic acid/ethylene-induced defense responses. In The Plant journal : for cell and molecular biology 61 (2), pp. 200-210. DOI: 10.1111/j.1365-313X.2009.04044.x.

Zander, Mark; Thurow, Corinna; Gatz, Christiane (2014): TGA Transcription Factors Activate the Salicylic Acid-Suppressible Branch of the Ethylene-Induced Defense Program by Regulating ORA59 Expression. In Plant physiology 165 (4), pp. 1671-1683. DOI: 10.1104/pp.114.243360.

Zeilmaker, Tieme; Ludwig, Nora R.; Elberse, Joyce; Seidl, Michael F.; Berke, Lidija; van Doorn, Arjen et al. (2015): DOWNY MILDEW RESISTANT 6 and DMR6-LIKE OXYGENASE 1 are partially redundant but 
distinct suppressors of immunity in Arabidopsis. In The Plant journal : for cell and molecular biology 81 (2), pp. 210-222. DOI: 10.1111/tpj.12719.

Zhang, Kewei; Halitschke, Rayko; Yin, Changxi; Liu, Chang-Jun; Gan, Su-Sheng (2013): Salicylic acid 3hydroxylase regulates Arabidopsis leaf longevity by mediating salicylic acid catabolism. In Proceedings of the National Academy of Sciences of the United States of America 110 (36), pp. 14807-14812. DOI: 10.1073/pnas.1302702110.

Zhang, Yaxi; Xu, Shaohua; Ding, Pingtao; Wang, Dongmei; Cheng, Yu Ti; He, Jing et al. (2010): Control of salicylic acid synthesis and systemic acquired resistance by two members of a plant-specific family of transcription factors. In Proceedings of the National Academy of Sciences of the United States of America 107 (42), pp. 18220-18225. DOI: 10.1073/pnas.1005225107.

Zhang, Yuelin; Fan, Weihua; Kinkema, Mark; Li, Xin; Dong, Xinnian (1999): Interaction of NPR1 with basic leucine zipper protein transcription factors that bind sequences required for salicylic acid induction of the PR-1 gene. In Proceedings of the National Academy of Sciences of the United States of America 96, pp. 6523-6528.

Zhang, Yuelin; Tessaro, Mark J.; Lassner, Michael; Li, Xin (2003): Knockout analysis of Arabidopsis transcription factors TGA2, TGA5, and TGA6 reveals their redundant and essential roles in systemic acquired resistance. In The Plant cell 15 (11), pp. 2647-2653. DOI: 10.1105/tpc.014894. 


\section{Abbreviations}

\begin{tabular}{|c|c|}
\hline$\%$ & Per cent \\
\hline${ }^{\circ} \mathrm{C}$ & Degree Celsius \\
\hline$\infty$ & infinity \\
\hline A. thaliana & Arabidopsis thaliana \\
\hline A. tumefaciens & Agrobacterium tumefaciens \\
\hline ALD1 & AGD2-LIKE DEFENSE RESPONSE PROTEIN 1 \\
\hline ANOVA & Analysis of Variance \\
\hline as-1 & Activation sequence-1 \\
\hline ATH1 & ARABIDOPSIS THALIANA HOMEOBOX GENE 1 \\
\hline ATP & Adenosine triphosphate \\
\hline BGL2 & $\beta-1,3-G L U C A N A S E$ \\
\hline BOP & BLADE-ON-PETIOLE \\
\hline $\mathrm{bp}$ & Base pair \\
\hline BTB/POZ & Broad-Complex, Tramtrack, and Bric-a-Brac/POX virus and Zinc finger \\
\hline bZIP & Basic domain/leucine zipper \\
\hline c & Centi $\left(10^{-2}\right)$ \\
\hline C, Cys & Cysteine residue \\
\hline CaMV & Cauliflower Mosaic Virus \\
\hline Cas & CRISPR-associated \\
\hline CC- & Coiled-Coil \\
\hline cDNA & Coding DNA \\
\hline $\mathrm{Col}-\mathrm{O}$ & Colombia-0 (Arabidopsis thaliana ecotype), wild type \\
\hline CRISPR & Clustered regularly interspaced short palindromic repeats \\
\hline cr-RNA & CRISPR RNA \\
\hline dATP & Deoxy Adenosine Triphosphate \\
\hline dCTP & Deoxy Cytidine Triphosphate \\
\hline DEG & Differentially Expressed Genes \\
\hline dGTP & Deoxy Guanosine Triphosphate \\
\hline DLO1 & DMR6-LIKE OXYGENASE 1 \\
\hline DMR6 & DOWNY MILDEW RESISTANT 6 \\
\hline DNA & Deoxyribonucleic Acid \\
\hline dNTPs & Deoxyribonucteotides \\
\hline dTTP & Deoxy Tymidine Triphosphate \\
\hline dYT & Yeast extract and Tryptone media \\
\hline E. coli & Escherichia coli \\
\hline EDS5 & ENHANCED DISEASE SUSCEPTIBILITY 5 \\
\hline EDTA & Ethylene Diamine Tetra-acetic Acid \\
\hline ET & Ethylene \\
\hline ETI & Effector triggered immunity \\
\hline
\end{tabular}




\begin{tabular}{|c|c|}
\hline EtOH & Ethanol \\
\hline ETS & Effector triggered susceptibility \\
\hline EV & Empty Vector \\
\hline $\mathrm{F}$ & Faraday \\
\hline FDR & False Discovery Rate \\
\hline FeS & Iron Sulfur \\
\hline FMO1 & FLAVIN-DEPENDENT MONOOXYGENASE 1 \\
\hline fwd & Forward \\
\hline g & Gram \\
\hline GFP & Green Fluorescent Protein \\
\hline GO & Gene ontology \\
\hline gRNA & guide RNA \\
\hline GRX & Glutaredoxin \\
\hline GSH & Glutathione \\
\hline GSNO & S-Nitrosoglutathione \\
\hline GUS & $\beta$-glucuronidase \\
\hline $\mathrm{HA}$ & Human influenca hemagglutinin \\
\hline HR & Hypersensitive Response \\
\hline INA & 2,6-dichloroisonicotinic acid \\
\hline$J A$ & Jasmonic acid \\
\hline $\mathrm{k}$ & Kilo $\left(10^{3}\right)$ \\
\hline L & Liter \\
\hline $\mathrm{L}^{-1}$ & Per liter \\
\hline LD & Long Day \\
\hline LRR & Leucine-Rich Repeat \\
\hline $\mathrm{m}$ & Meter \\
\hline $\mathrm{m}$ & Mili $\left(10^{-3}\right)$ \\
\hline $\mathrm{M}$ & Molarity (mol per liter) \\
\hline MAMP & Microbial Associated Molecular Patterns \\
\hline $\min$ & Minute \\
\hline $\mathrm{n}$ & Nano $\left(10^{-9}\right)$ \\
\hline NADPH & Nicotinamide Adenine Dinucleotide Phosphate \\
\hline NB & Nucleotide-Binding \\
\hline NHP & N-hydroxypipecolic acid \\
\hline NINJA & NOVEL INTERACTOR OF JAZ \\
\hline NO & Nitric Oxide \\
\hline NOS & Reactive Nitrogen Species \\
\hline NPR1 & NONEXPRESSOR OF PATHOGENESIS-RELATED GENES 1 \\
\hline NRT & NITRATE TRANSPORTER \\
\hline OD600 & Optical density at wavelength $600 \mathrm{~nm}$ \\
\hline$p$ & $\begin{array}{l}P \text {-value (probability of obtaining a test statistic assuming that the nul } \\
\text { hypothesis is true) }\end{array}$ \\
\hline PAL & PHENYALANIN AMMONIA-LYASE \\
\hline
\end{tabular}




\begin{tabular}{|c|c|}
\hline PAM & Protospacer Adjacent Motif \\
\hline PAMP & Pathogen-Associated Molecular Patterns \\
\hline PAN & PERIANTHIA \\
\hline PBS3 & AVRPPHB SUSCEPTIBLE 3 \\
\hline PCA & Principal components analysis \\
\hline PCD & Programmed Cell Death \\
\hline PCR & Polymerase Chain Reaction \\
\hline Pip & Pipecolic acid \\
\hline PR1 & PATHOGENESIS RELATED 1 \\
\hline pre-crRNA & Precursor-CRISPR RNA \\
\hline PR-genes & PATHOGENESIS-RELATED genes \\
\hline pro & Promoter \\
\hline PRR & Pattern Recognition Receptors \\
\hline Psm & Pseudomonas syringae pathovar maculicola ES4326 \\
\hline PTI & PAMP triggered immunity \\
\hline qRT-PCR & Quantitative Real-Time PCR \\
\hline rev & Reverse \\
\hline R-gene & RESISTANCE gene \\
\hline RNA & Ribonucleic Acid \\
\hline ROS & Reactive Oxygen Species \\
\hline ROXY & CC-type glutaredoxin \\
\hline RPKM & Reads per kilobase million \\
\hline $\mathrm{rpm}$ & Rotations per minute \\
\hline RT & Room Temperature \\
\hline SA & Salicylic Acid \\
\hline SAR & Systemic Acquired Resistance \\
\hline SARD1 & SYSTEMIC ACQUIRED RESISTANCE DEFICIENT 1 \\
\hline SARD4 & SYSTEMIC ACQUIRED RESISTANCE DEFICIENT 4 \\
\hline SD & Short day \\
\hline SDS & Sodium Dodecyl Sulfate \\
\hline SDS-PAGE & SDS-Polyacrylamide Gel Electrophoresis \\
\hline sec & Second \\
\hline SEM & Standard Error of the Mean \\
\hline sgRNA & Single guide RNA \\
\hline snc1 & Suppressor of npr1-consititive 1 \\
\hline $\mathrm{t}$ & Terminator \\
\hline T3SS & Type three Secretion System \\
\hline T7E1 & T7 endonuclease 1 \\
\hline TGA & TGACGTCA cis-element-binding proteins \\
\hline TGA1g & TGA1 genomic \\
\hline TGA1gr & TGA1 genomic reduced \\
\hline TIR & Toll and Interleukin 1 Receptor proteins \\
\hline TPL & TOPLESS \\
\hline
\end{tabular}




\begin{tabular}{l|l}
\hline TPR & TPL-RELATED PROTEINS \\
\hline tracrRNA & Trans-activating RNA \\
\hline Tris & Tris-hydroxymethylamino methane \\
\hline TRX & THIOREDOXIN \\
\hline TSS & Transcription Start Site \\
\hline$U$ & Unit \\
\hline$U B Q$ & UBIQUITIN \\
\hline$V$ & Volt \\
\hline VP16 & Herpes simplex Virus Protein 16 \\
\hline$w / v$ & Weight per volume \\
\hline YEB & Yeast Extract Broth media \\
\hline$\alpha$ & Alpha, antibody \\
\hline$\beta$ & Beta \\
\hline$\lambda$ & Lambda virus \\
\hline$\mu$ & Micro (10-6) \\
\hline$\Omega$ & Ohm
\end{tabular}




\section{Supplementary Data}

\subsection{Supplementary Figures}

A

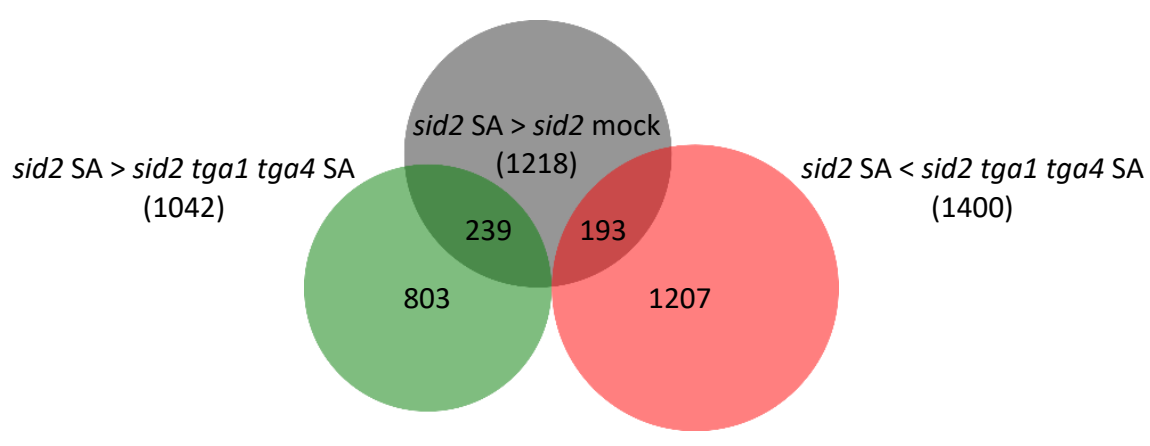

B

C

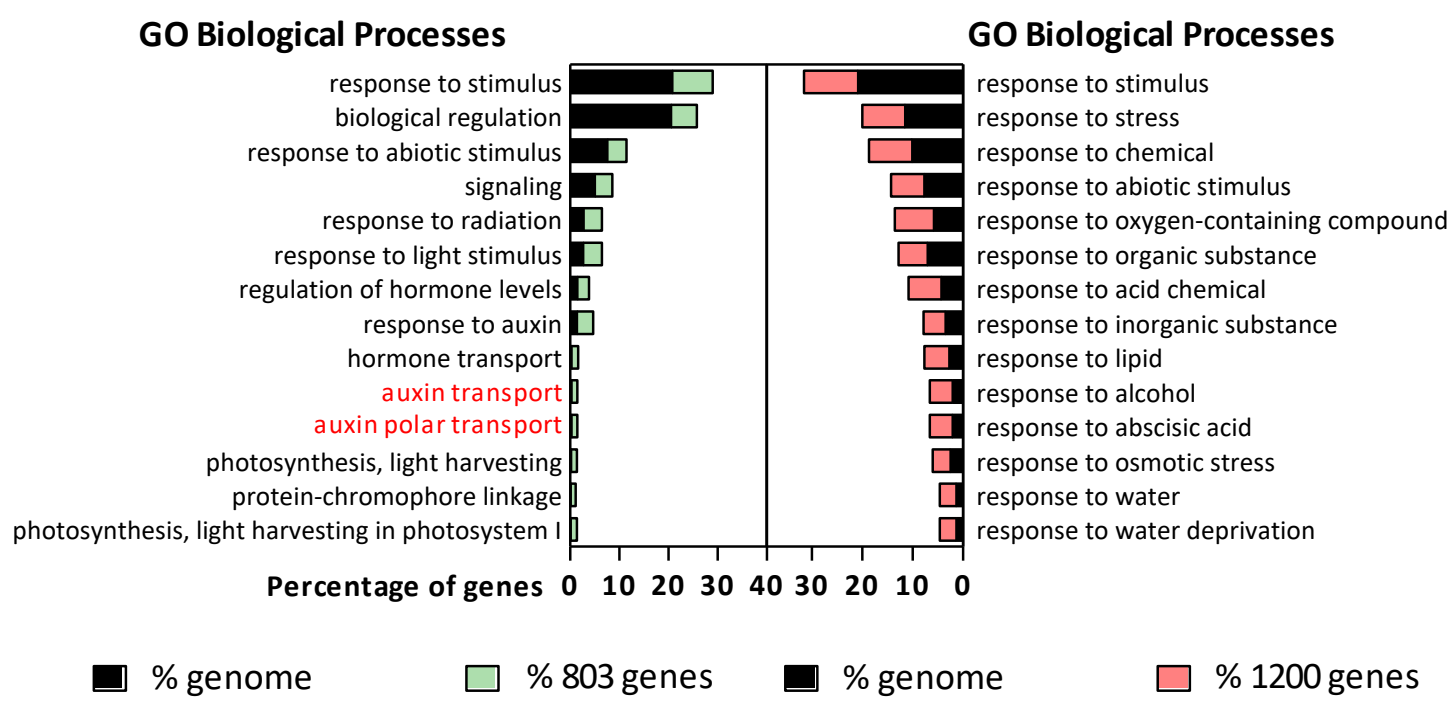

Supplementary Figure 1 Analysis of genes which were less and more expressed in sid2 tga1 tga4 mutant twenty-four hours after SA treatment comparing to SA-inducible genes in sid 2 background

A Venn diagram was used to analyze the relation of SA-inducible genes in sid2 (gray circle) and genes which were more (red circle) and less (green circle) expressed in sid 2 tga1 tga4 mutant after SA treatment.

B The group of 803 genes, which were not SA-inducible in sid 2 but were less expressed in the sid 2 tga 1 tga 4 mutant background was analyzed for the GO (Gene Ontology) enrichment in biological processes (light green bars). The Arabidopsis genome was used as a background set (black bars). Indicated in red are GO terms which were previously found to be downregulated in tga1 tga4 mutant (Li et al, 2019). 
E The group of 1200 genes, which were not SA-inducible in sid 2 but were more expressed in the sid2 tga1 tga 4 mutant background was analyzed for the GO (Gene Ontology) enrichment in biological processes (light red bars). The Arabidopsis genome was used as a background set (black bars).

Differentially expressed genes were determined as FC (fold change (log2 FC $\geq 1$ or $F C \leq-1), p<0.05$ ). Statistical analysis was performed using RobiNA software. Gene Ontology analysis platform was used for the GO enrichment analysis (http://geneontology.org/). The software uses Arabidopsis genome reference list consisting of 27581 genes. Statistical analysis was performed using Fisher test and False discovery rate $(F D R)<0.05(B), F D R<10^{-5}(C)$. Top 14 significant are shown.

A

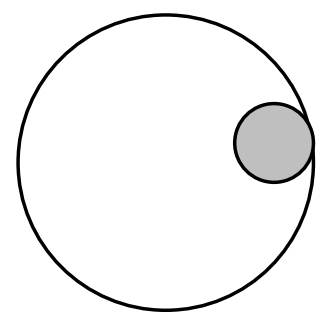

$7.8 \%$
B

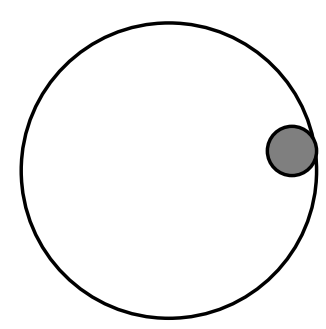

$4.4 \%$

\section{GO Biological Processes}

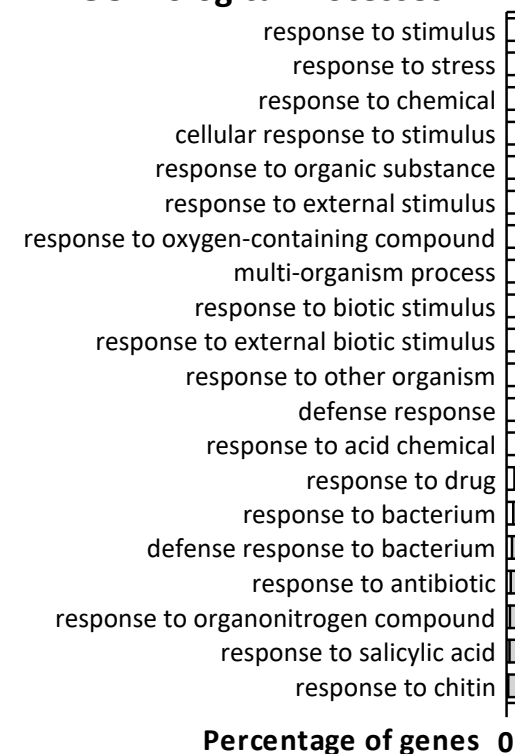

$\%$ in genome $\%$ in 2145 genes (8h)

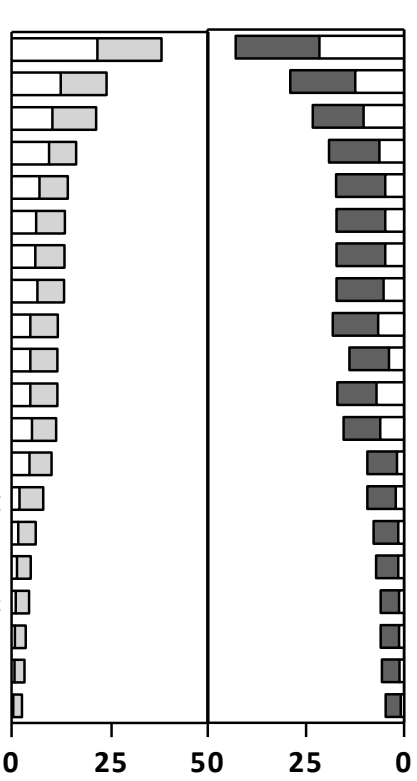

GO Biological Processes

response to stimulus response to stress response to chemical response to external stimulus response to biotic stimulus response to external biotic stimulus response to other organism defense response multi-organism process defense response to other organism response to organic substance response to oxygen-containing compound response to bacterium response to drug defense response to bacterium immune system process innate immune response immune response response to antibiotic response to salicylic acid

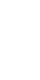


B $4.4 \%$ of Arabidopsis genome was induced twenty-four hour after SA treatment. The group of 1218 genes that were SA-inducible in sid2 after twenty-four hours was analyzed for the GO enrichment in biological processes using Arabidopsis genome as background.

Differentially expressed genes were determined as FC (fold change (log2 FC $\geq 1$ or $F C \leq-1), p<0.05$ ). Statistical analysis was performed using RobiNA software. Gene Ontology analysis platform was used for the GO enrichment analysis (http://geneontology.org/). The software uses Arabidopsis genome reference list consisting of 27581 genes. Statistical analysis was performed using Fisher test and False discovery rate $(F D R)<10^{-16}(A), F D R<10^{-19}(B)$. Top 20 most significant are shown.
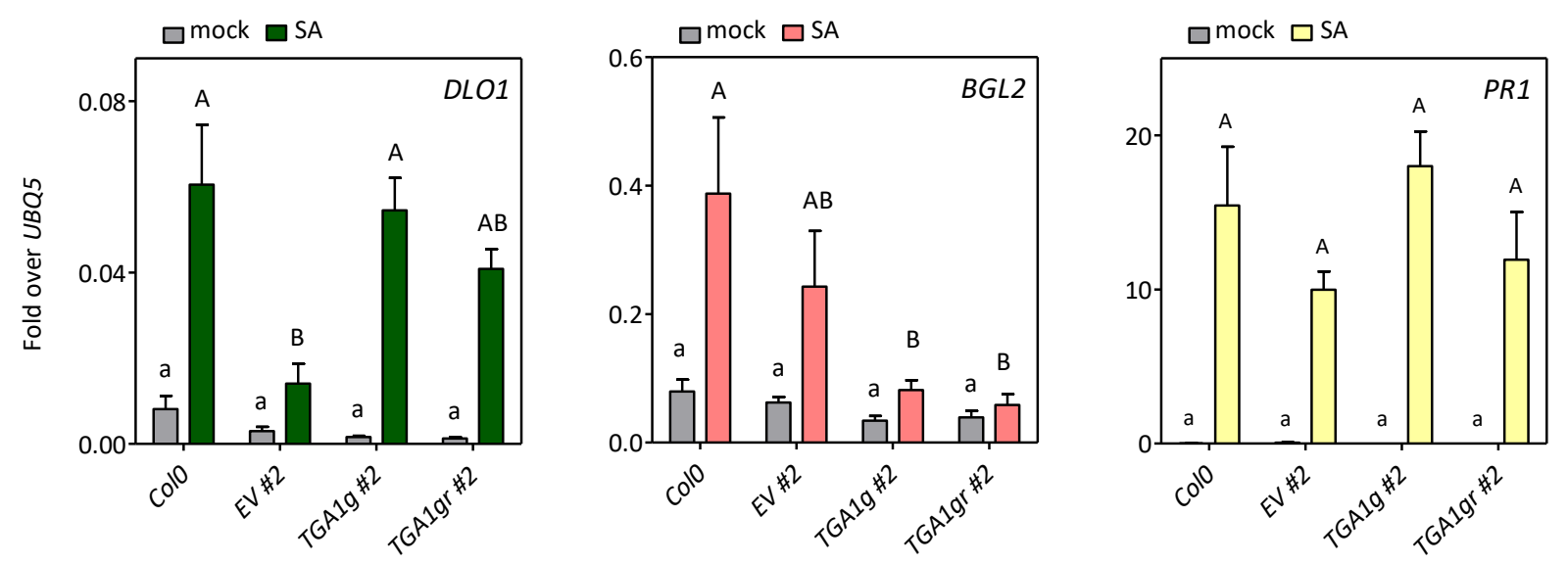

Supplementary Figure 3 Complementation of tga1 tga4 mutant is not influenced by the redox state of four critical cysteine residues.

qRT-PCR analysis of DLO1, BGL2 and PR1 transcript levels after SA treatment of wild-type and tga1 tga4 plants complimented either with empty vector (EV), TGA1 genomic clone (TGA1g) or TGA1 genomic clone carrying mutations in the four critical cysteine residues (TGA1gr) under native promoter. Four-week-old plants were sprayed either with mock or $1 \mathrm{mM} \mathrm{SA}$ at $1 \mathrm{~h}$ after the subjective dawn and further incubated for $8 \mathrm{~h}$. Transcript levels were normalized to transcript level of UBQ5. Bars represent the average \pm SEM of four to six plants of each genotype. Experiment was repeated once with similar results. All data shown here are from the same experiment.

Statistical analysis was performed using two-way ANOVA followed by Bonferroni's post-hoc test. Lowercase letters indicate significant differences $(P<0.05)$ between mock-treated samples; uppercase letters indicate significant differences $(\mathrm{P}<0.05)$ between SA-treated samples. $E V$-empty vector, TGA1TGA1 genomic clone, TGA1gr-TGA1 genomic clone with four cysteines mutated, mock-water, SAsalicylic acid. 
A
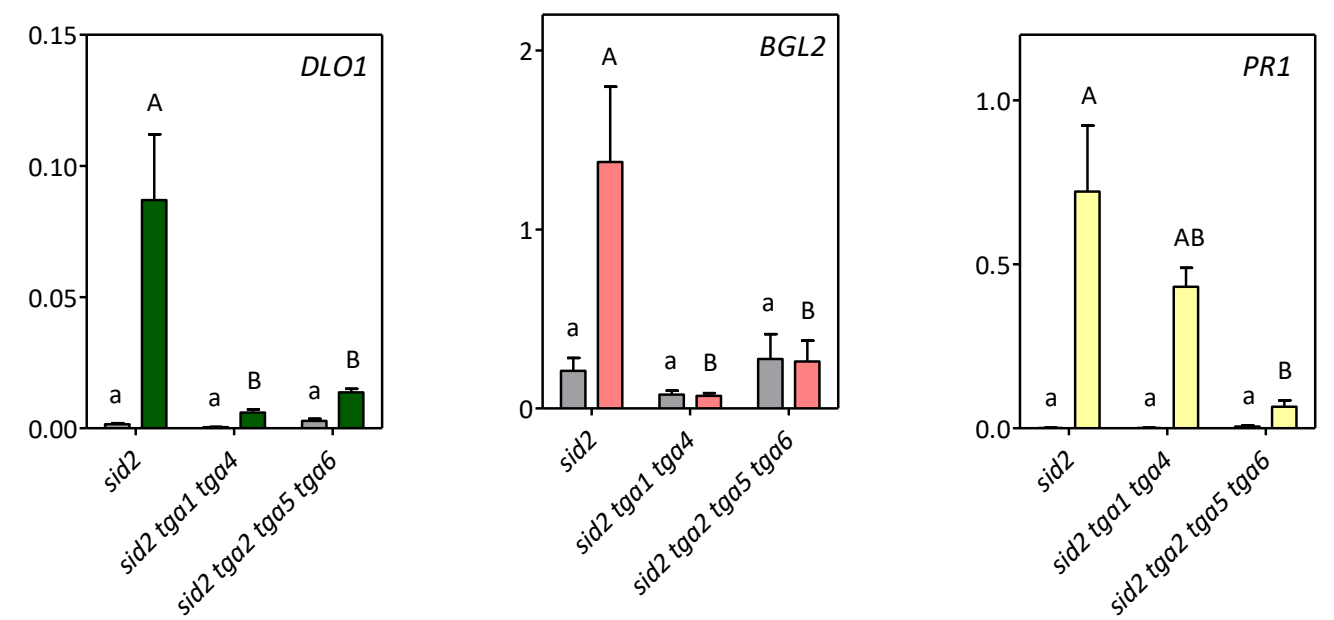

B
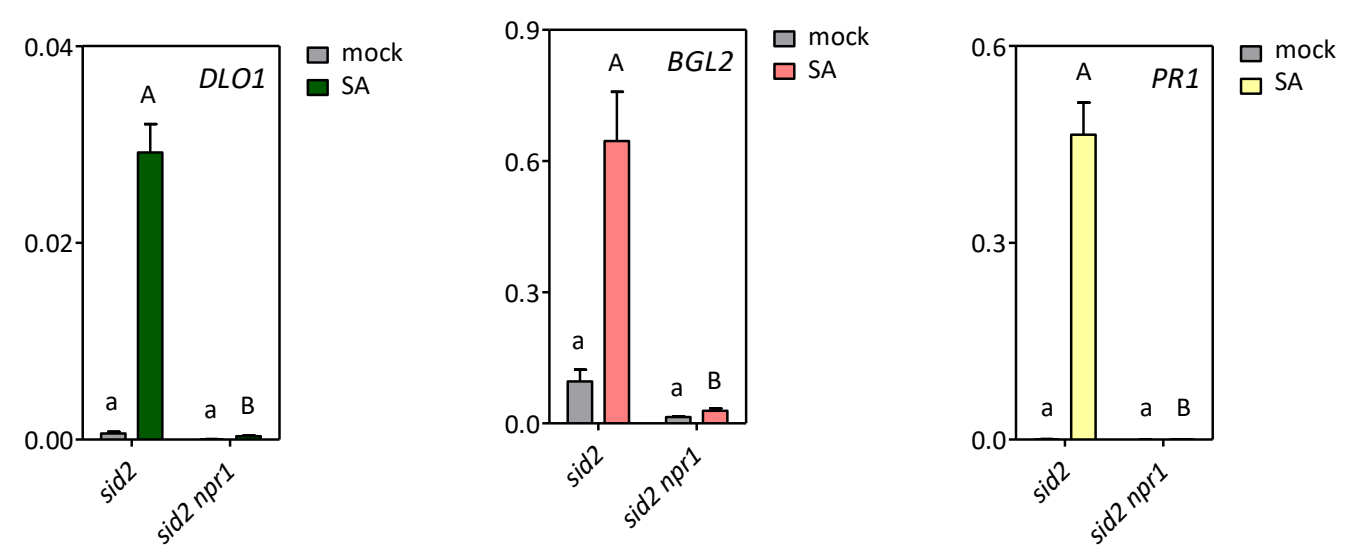

Supplementary Figure 4 Induction of $D L O 1, B G L 2$ and $P R 1$ genes after SA treatment is regulated by clade I and II TGA transcription factors and NPR1.

A qRT-PCR analysis of DLO1, BGL2 and PR1 transcript levels after SA treatment of sid2, sid2 tga1 tga4 and sid 2 tga 2 tga 5 tga6. Four-week-old plants were sprayed either with mock or $1 \mathrm{mM} \mathrm{SA}$ at $1 \mathrm{~h}$ after the subjective dawn and further incubated for $8 \mathrm{~h}$. Transcript levels were normalized to transcript level of UBQ5. Bars represent the average \pm SEM of four to six plants of each genotype. Experiment was repeated once with similar results. Shown data are taken from the same experiment.

B qRT-PCR analysis of DLO1, BGL2 and PR1 transcript levels after SA treatment of sid2 and sid2 npr1. Four-week-old plants were sprayed either with mock or $1 \mathrm{mM} \mathrm{SA}$ at $1 \mathrm{~h}$ after the subjective dawn and further incubated for $8 \mathrm{~h}$. Transcript levels were normalized to transcript level of UBQ5. Bars represent the average \pm SEM of four to seven plants of each genotype. Experiment was repeated once with similar results. Shown data are taken from the same experiment.

Statistical analysis was performed using two-way ANOVA followed by Bonferroni's post-hoc test. Lowercase letters indicate significant differences $(P<0.05)$ between mock-treated samples; uppercase letters indicate significant differences $(P<0.05)$ between SA-treated samples. mock-water, SA-salicylic acid 
A

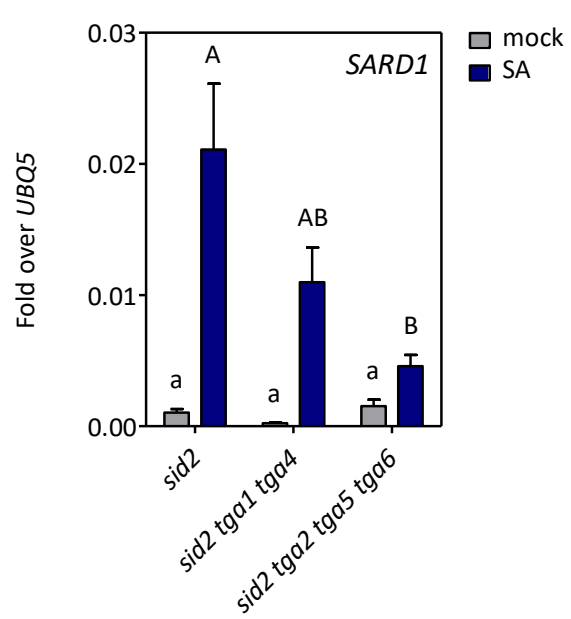

B

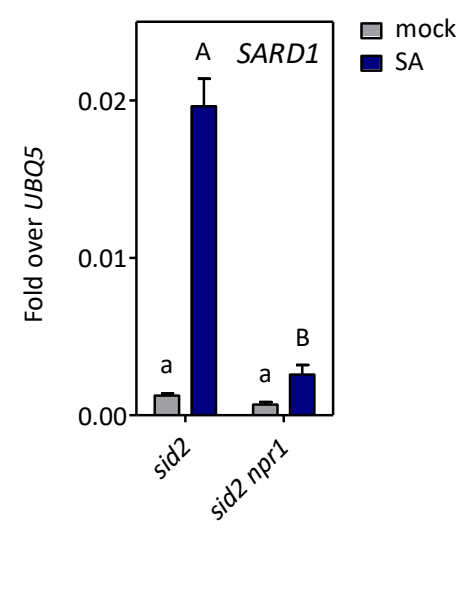

Supplementary Figure 5 TGA2/TGA5/TGA6 and NPR1 control SARD1 expression downstream of SA.

A qRT-PCR analysis of SARD 1 transcript levels after SA treatment of sid2, sid2 tga1 tga4 and sid2 tga 2 tga5 tga6. Four-week-old plants were sprayed either with mock or $1 \mathrm{mM} \mathrm{SA}$ at $1 \mathrm{~h}$ after the subjective dawn and further incubated for $8 \mathrm{~h}$. Transcript levels were normalized to transcript level of UBQ5. Bars represent the average \pm SEM of four to six plants of each genotype. Experiment was repeated once with similar results. Shown data are taken from the same experiment.

B qRT-PCR analysis of DLO1, BGL2 and PR1 transcript levels after SA treatment of sid2 and sid2 npr1. Four-week-old plants were sprayed either with mock or $1 \mathrm{mM} \mathrm{SA}$ at $1 \mathrm{~h}$ after the subjective dawn and further incubated for $8 \mathrm{~h}$. Transcript levels were normalized to transcript level of UBQ5. Bars represent the average \pm SEM of four to seven plants of each genotype. Experiment was repeated once with similar results. Shown data are taken from the same experiment

Statistical analysis was performed using two-way ANOVA followed by Bonferroni's post-hoc test. Lowercase letters indicate significant differences $(P<0.05)$ between mock-treated samples; uppercase letters indicate significant differences $(P<0.05)$ between SA-treated samples. mock-water, SA-salicylic acid 
A

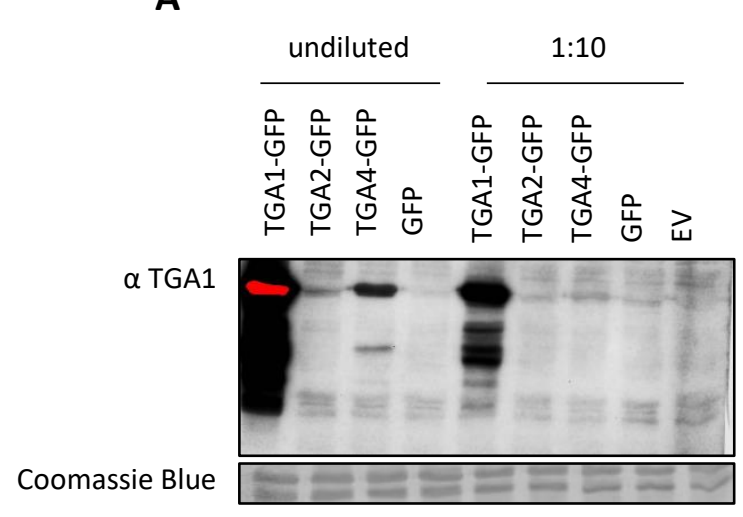

B

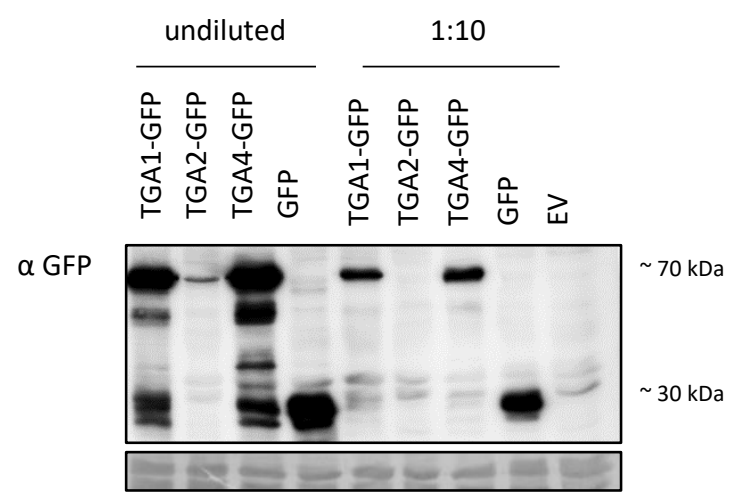

Supplementary Figure 6 TGA1 antibody is specific for TGA1 protein

Escherichia coli (BL1 strain) bacterium was transformed with a plasmid containing TGA1-GFP, TGA2GFP, TGA4-GFP and GFP gene regulated by a T7 promoter and an empty vector (EV) control plasmid. Isopropyl-ß-D-thiogalactopyranoside (IPTG) induces the expression by removing the lac-repressor on the T7 polymerase gene. T7 polymerase subsequently activates the T7 promoter. Bacterial cultures were harvested 3 hours after IPTG induction. Cells were sonicated and lysate was centrifuged. Supernatant was mixed with $4 \times$ SDS loading buffer. Diluted samples were mixed with $E$. coli expressing empty vector in 1 to 10 ratios.

A Western blot analysis of protein extracts of $E$. coli expressing TGA1-GFP, TGA2-GFP, TGA4-GFP and GFP gene regulated by a T7 promoter and an empty vector (EV). TGA1 protein was detected using TGA1 antibody.

B Western blot analysis of protein extracts of $E$. coli expressing TGA1-GFP, TGA2-GFP, TGA4-GFP and GFP gene regulated by a T7 promoter and an empty vector (EV). GFP protein fusions were detected using GFP antibody.

EV-empty vector, GFP-Green Fluorescent Protein, kDa-kilo Dalton. Experiment was performed by Dyari Mohammed. 


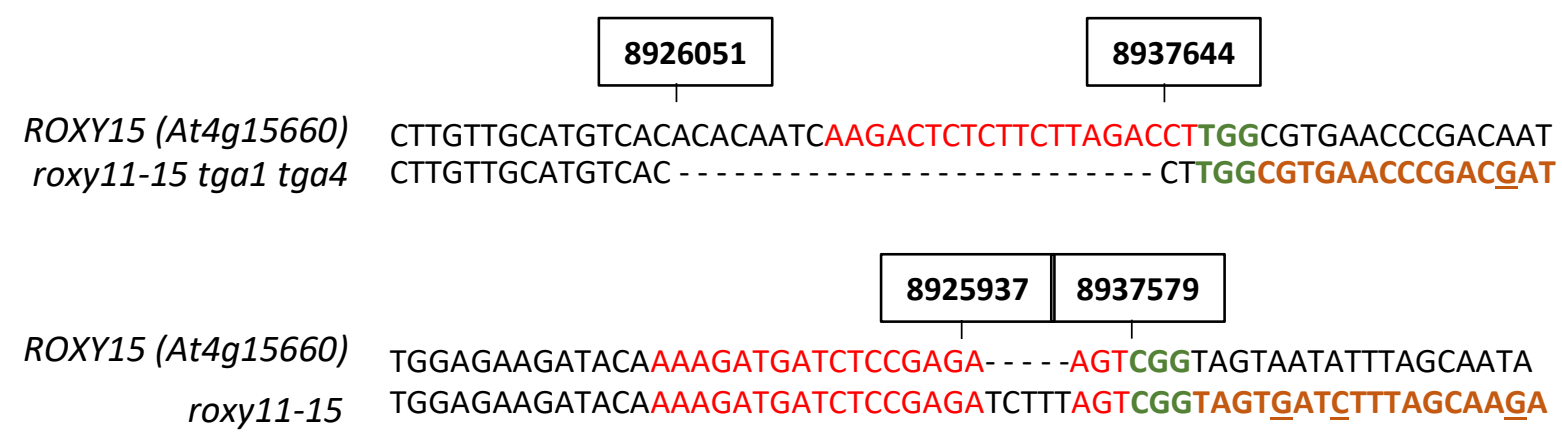

Supplementary Figure 7 Alignment of genomic ROXY15 with chimeric ROXY15 from roxy11-15 and roxy11-15 tga1 tga4 mutant generated by CRISPR-Cas9.

ROXY11-15 genes are organized in a fifteen kilobase long cluster at the chromosome four of Arabidopsis thaliana. We used CRISPR-Cas9 technology to knock out the whole cluster of genes. To do so, we designed three types of oligonucleotides, named A, B and C, each targeting different sets of genes from the cluster. The major goal of this CRISPR-Cas9 approach was to cause the deletion of five genes with oligonucleotides targeting the outermost genes ROXY15 and ROXY11.

roxy11-15 tga1 tga4 had a 26 base pair deletion upstream of PAM (green) sequence targeted by oligonucleotide (red) resulting in chimeric product of ROXY15 (black) and ROXY11 (brown) gene. roxy11-15 had a 5 base pair insertion upstream of PAM (green) sequence of oligonucleotide (red) resulting in chimeric product of ROXY15 (black) and ROXY11 (brown) gene. Nucleotide number of chromosome four of Arabidopsis thaliana is shown in black boxes above sequences. Single nucleotide differences between ROXY15 and ROXY11 genes are underlined. 
ROXY6 (At1g06830) ATGGACAAAGTTATGAGAATGTCGTCCGAAAAAGGGG- TGGTTATATTTACCAAGAGCTC roxy6 ATGGACAAAGTTATGAGAATGTCGTCCGAAAAAGGGGGTGGTTATATTTACCAAGAGCTC

ROXY7 (At2g30540) GTTGCATGTCCTATGCGGTCCAAGTACTTTTCCAAGACCTTGGGGTTCACCCAACAGTCCA roxy7 GTTGCATGTCCTATG- GGTCCAAGTACTTTTCCAAGACCTTGGGGTTCACCCAACAGTCCA
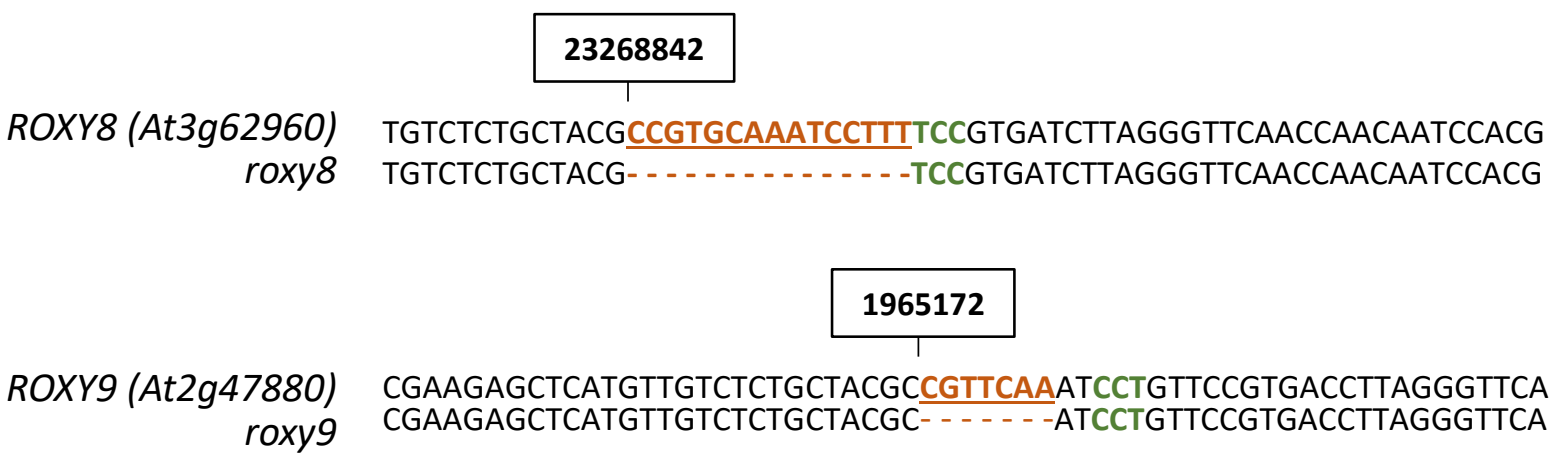

Supplementary Figure 8 Alignment of genomic ROXY6-9 with mutant alleles from roxy6-9 generated by CRISPR-Cas9.

roxy6 had a single base pair insertion upstream of PAM (green) sequence resulting in frame shift.

roxy7 had a single base pair deletion upstream of PAM (green) sequence resulting in frame shift.

roxy8 had 16 base pair deletion resulting in frame shift.

roxy 9 had 7 base pair deletion resulting in frame shift.

All frame shifts led to premature stop codon. Nucleotide number of chromosomes of Arabidopsis thaliana is shown in black boxes above sequences. Nucleotide differences between wild-type and mutated alleles are marked in brown and underlined. 


\subsection{Supplementary Tables}

Supplementary Table 1207 genes which are SA-inducible in sid2 and less expressed in sid2 tga1 tga4 after SA treatment for eight hours.

\begin{tabular}{|c|c|c|c|c|c|}
\hline \multicolumn{2}{|c|}{ Identification } & \multicolumn{2}{|c|}{ SA induction in sid 2} & \multicolumn{2}{|c|}{ sid2 tga1 tga4 vs sid2 (SA) } \\
\hline AGI code & Note & $\log _{2} \mathrm{FC}$ & p-value & $\log _{2} \mathrm{FC}$ & p-value \\
\hline AT3G13130 & transmembrane protein & 3,83 & 0,0156812 & $-4,62$ & 0,0037142 \\
\hline AT4G11070 & WRKY family transcription factor & 4,17 & 0,0030552 & $-4,46$ & 0,0023906 \\
\hline AT3G21520 & $\begin{array}{l}\text { transmembrane protein\%2C } \\
\text { putative (DUF679 domain } \\
\text { membrane protein 1) }\end{array}$ & 7,37 & $4,172 \times 10-07$ & $-3,27$ & 0,0011429 \\
\hline AT5G47850 & CRINKLY4 related 4 & 5,67 & $7,194 \times(10)-05$ & $-3,15$ & 0,0033905 \\
\hline AT4G23150 & $\begin{array}{l}\text { cysteine-rich RLK (RECEPTOR-like } \\
\text { protein kinase) } 7\end{array}$ & 3,77 & 0,0110347 & $-3,06$ & 0,016671 \\
\hline AT4G13890 & $\begin{array}{l}\text { Pyridoxal phosphate (PLP)- } \\
\text { dependent transferases } \\
\text { superfamily protein }\end{array}$ & 6,11 & $9,227 \times(10)-05$ & $-2,95$ & 0,0055005 \\
\hline AT4G23140 & $\begin{array}{l}\text { cysteine-rich RLK (RECEPTOR-like } \\
\text { protein kinase) } 6\end{array}$ & 2,99 & 0,0005327 & $-2,88$ & 0,000243 \\
\hline AT4G15270 & glucosyltransferase-like protein & 4,39 & 0,0017018 & $-2,87$ & 0,015952 \\
\hline AT4G19970 & $\begin{array}{l}\text { nucleotide-diphospho-sugar } \\
\text { transferase family protein }\end{array}$ & 5,47 & $1,744 \times(10)-10$ & $-2,85$ & $1,98 \times(10)-06$ \\
\hline AT4G10500 & $\begin{array}{l}\text { 2-oxoglutarate (2OG) and Fe(II)- } \\
\text { dependent oxygenase superfamily } \\
\text { protein }\end{array}$ & 4,93 & 0,0014105 & $-2,80$ & 0,0211106 \\
\hline AT3G24900 & receptor like protein 39 & 3,63 & 0,0090811 & $-2,79$ & 0,025206 \\
\hline AT5G24540 & beta glucosidase 31 & 4,81 & $4,006 \times(10)-05$ & $-2,73$ & 0,0045573 \\
\hline AT1G65484 & transmembrane protein & 2,32 & 0,0020778 & $-2,68$ & 0,0001567 \\
\hline AT3G21770 & Peroxidase superfamily protein & 1,14 & 0,0343554 & $-2,67$ & $1,496 \times(10)-05$ \\
\hline AT4G16260 & $\begin{array}{l}\text { Glycosyl hydrolase superfamily } \\
\text { protein }\end{array}$ & 6,98 & 0,000207 & $-2,63$ & 0,0457931 \\
\hline AT2G32680 & receptor like protein 23 & 3,17 & 0,0005875 & $-2,61$ & 0,0011654 \\
\hline AT2G29100 & glutamate receptor 2.9 & 6,08 & 0,0003037 & $-2,57$ & 0,0358677 \\
\hline AT4G35380 & $\begin{array}{l}\text { SEC7-like guanine nucleotide } \\
\text { exchange family protein }\end{array}$ & 2,45 & 0,000699 & $-2,54$ & 0,0006428 \\
\hline AT4G34380 & $\begin{array}{l}\text { Transducin/WD40 repeat-like } \\
\text { superfamily protein }\end{array}$ & 2,85 & 0,0120157 & $-2,48$ & 0,0220134 \\
\hline AT3G25010 & receptor like protein 41 & 3,54 & 0,0102415 & $-2,48$ & 0,0331024 \\
\hline AT5G22530 & hypothetical protein & 5,74 & $1,576 \times(10)-11$ & $-2,32$ & $7,119 \times(10)-05$ \\
\hline AT1G26380 & $\begin{array}{l}\text { FAD-binding Berberine family } \\
\text { protein }\end{array}$ & 5,07 & 0,0008618 & $-2,31$ & 0,0364358 \\
\hline AT3G60470 & $\begin{array}{l}\text { transmembrane protein } \% 2 C \\
\text { putative (DUF247) }\end{array}$ & 6,03 & $6,47 \times(10)-18$ & $-2,30$ & $9,019 \times(10)-07$ \\
\hline AT2G43000 & NAC domain containing protein 42 & 5,19 & $2,663 \times(10)-07$ & $-2,20$ & 0,003876 \\
\hline AT5G24240 & $\begin{array}{l}\text { phosphatidylinositol 4-kinase } \\
\text { gamma-like protein }\end{array}$ & 3,89 & $2,117 \times(10)-05$ & $-2,18$ & 0,0056948 \\
\hline AT5G56960 & $\begin{array}{l}\text { basic helix-loop-helix (bHLH) DNA- } \\
\text { binding family protein }\end{array}$ & 4,64 & 0,0006323 & $-2,18$ & 0,0292528 \\
\hline AT5G38250 & Protein kinase family protein & 4,60 & $4,246 \times(10)-05$ & $-2,18$ & 0,0096756 \\
\hline AT3G15518 & hypothetical protein & 4,13 & 0,0006077 & $-2,18$ & 0,0306736 \\
\hline
\end{tabular}




\begin{tabular}{|c|c|c|c|c|c|}
\hline AT4G18430 & RAB GTPase homolog A1E & 6,71 & $4,74 \times(10)-08$ & $-2,16$ & 0,0065938 \\
\hline AT2G02930 & glutathione S-transferase F3 & 3,35 & $1,177 \times(10)-05$ & $-2,16$ & 0,0005087 \\
\hline AT5G38310 & hypothetical protein & 3,07 & 0,0002048 & $-2,15$ & 0,0037925 \\
\hline AT5G18470 & $\begin{array}{l}\text { Curculin-like (mannose-binding) } \\
\text { lectin family protein }\end{array}$ & 3,94 & $2,392 \times(10)-05$ & $-2,15$ & 0,0052902 \\
\hline AT3G62990 & $\begin{array}{l}\text { myelin transcription factor-like } \\
\text { protein }\end{array}$ & 5,50 & 0,0035274 & $-2,12$ & 0,0378565 \\
\hline AT1G14080 & fucosyltransferase 6 & 4,86 & 0,0008758 & $-2,11$ & 0,0432473 \\
\hline AT3G56500 & serine-rich protein-like protein & 4,39 & $3,612 \times(10)-07$ & $-2,11$ & 0,0016676 \\
\hline AT5G10760 & $\begin{array}{l}\text { Eukaryotic aspartyl protease family } \\
\text { protein }\end{array}$ & 2,72 & $2,03 \times(10)-07$ & $-2,09$ & $4,442 \times(10)-06$ \\
\hline AT5G64550 & loricrin-like protein & 1,06 & 0,0014603 & $-2,08$ & $8,579 \times(10)-10$ \\
\hline AT5G44460 & calmodulin like 43 & 8,22 & $1,582 \times(10)-17$ & $-2,06$ & $7,054 \times(10)-05$ \\
\hline AT1G21310 & extensin 3 & 4,06 & $1,886 \times(10)-10$ & $-2,06$ & 0,0001088 \\
\hline AT1G12160 & $\begin{array}{l}\text { Flavin-binding monooxygenase } \\
\text { family protein }\end{array}$ & 2,24 & $2,145 \times(10)-05$ & $-2,03$ & $2,199 \times(10)-06$ \\
\hline AT1G13470 & hypothetical protein (DUF1262) & 4,39 & $2,48 \times(10)-13$ & $-2,03$ & $4,13 \times(10)-05$ \\
\hline AT5G66390 & Peroxidase superfamily protein & 2,24 & $1,088 \times(10)-06$ & $-2,02$ & $1,314 \times(10)-05$ \\
\hline AT4G23200 & $\begin{array}{l}\text { cysteine-rich RLK (RECEPTOR-like } \\
\text { protein kinase) } 12\end{array}$ & 2,20 & 0,0094722 & $-2,01$ & 0,0095225 \\
\hline AT5G11920 & 6-\%261-fructan exohydrolase & 5,00 & $4,417 \times(10)-08$ & $-1,99$ & 0,0048626 \\
\hline AT4G21926 & hypothetical protein & 1,70 & 0,0191399 & $-1,99$ & 0,0227745 \\
\hline AT1G21110 & O-methyltransferase family protein & 2,77 & 0,0001323 & $-1,99$ & 0,0024611 \\
\hline AT5G38900 & Thioredoxin superfamily protein & 6,10 & $1,211 \times(10)-05$ & $-1,98$ & 0,0495583 \\
\hline AT4G04540 & $\begin{array}{l}\text { cysteine-rich RLK (RECEPTOR-like } \\
\text { protein kinase) } 39\end{array}$ & 4,67 & $2,31 \times(10)-06$ & $-1,98$ & 0,0052368 \\
\hline AT5G65210 & $\begin{array}{l}\text { bZIP transcription factor family } \\
\text { protein }\end{array}$ & 1,31 & 0,0001641 & $-1,98$ & $2,858 \times(10)-09$ \\
\hline AT1G12940 & nitrate transporter 2.5 & 4,33 & $4,125 \times(10)-07$ & $-1,96$ & 0,0059527 \\
\hline AT3G13850 & LOB domain-containing protein 22 & 1,76 & 0,0011598 & $-1,95$ & 0,0039612 \\
\hline AT1G74140 & $\begin{array}{l}\text { Rhomboid-related intramembrane } \\
\text { serine protease family protein }\end{array}$ & 6,25 & $8,021 \times(10)-11$ & $-1,94$ & 0,0036968 \\
\hline AT3G15356 & Legume lectin family protein & 4,21 & $1,964 \times(10)-06$ & $-1,93$ & 0,0053166 \\
\hline AT1G67810 & sulfur E2 & 5,03 & $6,399 \times(10)-08$ & $-1,89$ & 0,0067711 \\
\hline AT1G02920 & glutathione S-transferase 7 & 5,15 & $9,347 \times(10)-07$ & $-1,89$ & 0,014685 \\
\hline AT1G14540 & Peroxidase superfamily protein & 6,44 & $4,799 \times(10)-14$ & $-1,89$ & 0,0002486 \\
\hline AT3G16530 & Legume lectin family protein & 3,17 & $5,376 \times(10)-05$ & $-1,89$ & 0,0026181 \\
\hline AT3G45860 & $\begin{array}{l}\text { cysteine-rich RLK (RECEPTOR-like } \\
\text { protein kinase) } 4\end{array}$ & 1,48 & 0,0365017 & $-1,88$ & 0,0035643 \\
\hline AT1G76960 & transmembrane protein & 3,10 & 0,0001048 & $-1,87$ & 0,0083992 \\
\hline AT1G51890 & $\begin{array}{l}\text { Leucine-rich repeat protein kinase } \\
\text { family protein }\end{array}$ & 5,61 & $1,001 \times(10)-06$ & $-1,87$ & 0,0260717 \\
\hline AT5G22520 & hypothetical protein & 3,88 & 0,0007381 & $-1,87$ & 0,0264465 \\
\hline AT5G24110 & WRKY DNA-binding protein 30 & 4,27 & $3,21 \times(10)-07$ & $-1,86$ & 0,0025829 \\
\hline AT1G58390 & $\begin{array}{l}\text { Disease resistance protein (CC-NBS- } \\
\text { LRR class) family }\end{array}$ & 2,73 & 0,0082365 & $-1,85$ & 0,0206837 \\
\hline AT4G18250 & $\begin{array}{l}\text { receptor Serine/Threonine kinase- } \\
\text { like protein }\end{array}$ & 4,57 & $3,943 \times(10)-15$ & $-1,85$ & $3,133 \times(10)-05$ \\
\hline AT3G28580 & $\begin{array}{l}\text { P-loop containing nucleoside } \\
\text { triphosphate hydrolases } \\
\text { superfamily protein }\end{array}$ & 4,23 & $1,595 \times(10)-05$ & $-1,84$ & 0,0144474 \\
\hline
\end{tabular}




\begin{tabular}{|c|c|c|c|c|c|}
\hline AT2G04450 & nudix hydrolase homolog 6 & 6,06 & $2,433 \times(10)-09$ & $-1,84$ & 0,0158594 \\
\hline AT5G47220 & $\begin{array}{l}\text { ethylene responsive element } \\
\text { binding factor } 2\end{array}$ & 2,59 & 0,0004058 & $-1,83$ & 0,0044622 \\
\hline AT1G76800 & $\begin{array}{l}\text { Vacuolar iron transporter (VIT) } \\
\text { family protein }\end{array}$ & 1,17 & 0,0482251 & $-1,82$ & 0,0056609 \\
\hline AT2G15220 & $\begin{array}{l}\text { Plant basic secretory protein (BSP) } \\
\text { family protein }\end{array}$ & 1,71 & 0,0183084 & $-1,81$ & 0,0043837 \\
\hline AT3G45290 & $\begin{array}{l}\text { Seven transmembrane MLO family } \\
\text { protein }\end{array}$ & 2,38 & $4,999 \times(10)-11$ & $-1,81$ & $2,713 \times(10)-08$ \\
\hline AT1G66465 & transmembrane protein & 3,47 & 0,000611 & $-1,79$ & 0,0235156 \\
\hline AT5G07760 & $\begin{array}{l}\text { formin homology } 2 \text { domain- } \\
\text { containing protein / FH2 domain- } \\
\text { containing protein }\end{array}$ & 3,72 & $8,014 \times(10)-07$ & $-1,78$ & 0,0013157 \\
\hline AT4G03450 & Ankyrin repeat family protein & 3,56 & $3,286 \times(10)-07$ & $-1,76$ & 0,0030628 \\
\hline AT4G22710 & $\begin{array}{l}\text { cytochrome P450\%2C family } \\
706 \% 2 \text { C subfamily A\%2C } \\
\text { polypeptide } 2\end{array}$ & 2,30 & $8,295 \times(10)-07$ & $-1,74$ & $2,309 \times(10)-05$ \\
\hline AT1G02930 & glutathione S-transferase 6 & 5,70 & $8,154 \times(10)-09$ & $-1,73$ & 0,0160953 \\
\hline AT1G35210 & hypothetical protein & 3,14 & $2,261 \times(10)-05$ & $-1,71$ & 0,0061621 \\
\hline AT3G57240 & beta-1\%2C3-glucanase 3 & 4,41 & $2,146 \times(10)-09$ & $-1,71$ & 0,0059765 \\
\hline AT3G02840 & ARM repeat superfamily protein & 5,91 & $2,007 \times(10)-08$ & $-1,69$ & 0,016496 \\
\hline AT2G15390 & fucosyltransferase 4 & 3,07 & 0,0001271 & $-1,68$ & 0,0080676 \\
\hline AT1G11125 & hypothetical protein & 1,35 & 0,0217496 & $-1,67$ & 0,0068445 \\
\hline AT5G37600 & hypothetical protein & 2,15 & $6,366 \times(10)-09$ & $-1,65$ & $1,622 \times(10)-06$ \\
\hline AT4G19370 & $\begin{array}{l}\text { chitin synthase } \% 2 C \text { putative } \\
\text { (DUF1218) }\end{array}$ & 3,74 & $2,082 \times(10)-05$ & $-1,65$ & 0,0174459 \\
\hline AT4G11470 & $\begin{array}{l}\text { cysteine-rich RLK (RECEPTOR-like } \\
\text { protein kinase) } 31\end{array}$ & 3,33 & $5,246 \times(10)-05$ & $-1,64$ & 0,0069021 \\
\hline AT2G06185 & & 3,75 & $3,111 \times(10)-10$ & $-1,63$ & 0,0024732 \\
\hline AT4G08300 & $\begin{array}{l}\text { nodulin MtN21 /EamA-like } \\
\text { transporter family protein }\end{array}$ & 1,46 & 0,0005288 & $-1,63$ & $5,146 \times(10)-05$ \\
\hline AT3G47050 & Glycosyl hydrolase family protein & 8,40 & $5,618 \times(10)-21$ & $-1,63$ & 0,0007805 \\
\hline AT2G26390 & $\begin{array}{l}\text { Serine protease inhibitor (SERPIN) } \\
\text { family protein }\end{array}$ & 3,85 & 0,0003356 & $-1,61$ & 0,0246111 \\
\hline AT1G33790 & jacalin lectin family protein & 1,36 & 0,0040581 & $-1,61$ & 0,0002922 \\
\hline AT4G18253 & $\begin{array}{l}\text { receptor Serine/Threonine kinase- } \\
\text { like protein }\end{array}$ & 3,67 & $4,567 \times(10)-08$ & $-1,61$ & 0,0028741 \\
\hline AT5G55450 & $\begin{array}{l}\text { Bifunctional inhibitor/lipid-transfer } \\
\text { protein/seed storage } 2 \mathrm{~S} \text { albumin } \\
\text { superfamily protein }\end{array}$ & 3,19 & $6,283 \times(10)-06$ & $-1,61$ & 0,0075276 \\
\hline AT5G47130 & Bax inhibitor-1 family protein & 1,66 & $2,031 \times(10)-05$ & $-1,61$ & $8,913 \times(10)-05$ \\
\hline AT1G67000 & Protein kinase superfamily protein & 1,95 & 0,0009783 & $-1,60$ & 0,0017296 \\
\hline AT4G15417 & RNAse II-like 1 & 7,32 & $3,936 \times(10)-07$ & $-1,58$ & 0,0343237 \\
\hline AT1G26420 & $\begin{array}{l}\text { FAD-binding Berberine family } \\
\text { protein }\end{array}$ & 5,66 & $1,141 \times(10)-06$ & $-1,58$ & 0,0407873 \\
\hline AT1G30370 & $\begin{array}{l}\text { alpha/beta-Hydrolases superfamily } \\
\text { protein }\end{array}$ & 4,43 & $4,065 \times(10)-08$ & $-1,58$ & 0,0124057 \\
\hline AT3G23120 & receptor like protein 38 & 1,36 & 0,0044552 & $-1,57$ & 0,006309 \\
\hline AT3G26320 & $\begin{array}{l}\text { cytochrome } \mathrm{P} 450 \% 2 \mathrm{C} \text { family } \\
71 \% 2 \mathrm{C} \text { subfamily } \mathrm{B} \% 2 \mathrm{C} \\
\text { polypeptide } 36\end{array}$ & 1,46 & $5,084 \times(10)-05$ & $-1,55$ & $5,343 \times(10)-07$ \\
\hline AT3G09405 & Pectinacetylesterase family protein & 3,38 & 0,0003413 & $-1,53$ & 0,0367592 \\
\hline AT3G07520 & glutamate receptor 1.4 & 1,23 & 0,0336689 & $-1,53$ & 0,002948 \\
\hline
\end{tabular}




\begin{tabular}{|c|c|c|c|c|c|}
\hline AT5G27420 & carbon/nitrogen insensitive 1 & 3,17 & $3,192 \times(10)-05$ & $-1,52$ & 0,0204087 \\
\hline AT4G22840 & Sodium Bile acid symporter family & 1,06 & 0,000568 & $-1,52$ & $9,271 \times(10)-07$ \\
\hline AT3G48630 & hypothetical protein & 5,17 & $3,613 \times(10)-07$ & $-1,52$ & 0,0361361 \\
\hline AT5G61070 & $\begin{array}{l}\text { histone deacetylase of the } \\
\text { RPD3/HDA1 superfamily } 18\end{array}$ & 1,84 & $4,691 \times(10)-05$ & $-1,52$ & 0,0010144 \\
\hline AT3G11010 & receptor like protein 34 & 2,21 & $2,043 \times(10)-05$ & $-1,52$ & 0,0055797 \\
\hline AT3G28890 & receptor like protein 43 & 2,74 & $3,169 \times(10)-08$ & $-1,50$ & 0,0008683 \\
\hline AT5G07780 & $\begin{array}{l}\text { Actin-binding FH2 (formin } \\
\text { homology 2) family protein }\end{array}$ & 1,82 & 0,0007281 & $-1,49$ & 0,0049687 \\
\hline AT5G57220 & $\begin{array}{l}\text { cytochrome P450\%2C family } \\
81 \% 2 \mathrm{C} \text { subfamily F\%2C polypeptide } \\
2\end{array}$ & 1,70 & 0,0002408 & $-1,49$ & 0,0003919 \\
\hline AT1G33030 & O-methyltransferase family protein & 5,45 & $2,467 \times(10)-08$ & $-1,49$ & 0,0212972 \\
\hline AT2G43140 & $\begin{array}{l}\text { basic helix-loop-helix (bHLH) DNA- } \\
\text { binding superfamily protein }\end{array}$ & 4,85 & $6,028 \times(10)-18$ & $-1,49$ & 0,0002508 \\
\hline AT4G23280 & $\begin{array}{l}\text { cysteine-rich RLK (RECEPTOR-like } \\
\text { protein kinase) } 20\end{array}$ & 4,18 & $3,738 \times(10)-06$ & $-1,48$ & 0,0244202 \\
\hline AT1G74590 & glutathione S-transferase TAU 10 & 1,74 & 0,0022598 & $-1,47$ & 0,0062441 \\
\hline AT3G50930 & cytochrome BC1 synthesi & 2,99 & 0,0003257 & $-1,46$ & 0,0318143 \\
\hline AT1G77200 & $\begin{array}{l}\text { Integrase-type DNA-binding } \\
\text { superfamily protein }\end{array}$ & 1,54 & 0,0277759 & $-1,46$ & 0,0427121 \\
\hline AT5G18661 & transmembrane protein & 4,09 & $9,151 \times(10)-06$ & $-1,45$ & 0,0444811 \\
\hline AT4G02520 & glutathione S-transferase PHI 2 & 4,92 & $4,487 \times(10)-08$ & $-1,45$ & 0,0394074 \\
\hline AT1G49000 & transmembrane protein & 4,47 & $1,513 \times(10)-06$ & $-1,45$ & 0,0489093 \\
\hline AT1G24140 & Matrixin family protein & 2,36 & 0,000645 & $-1,43$ & 0,0266724 \\
\hline AT4G14450 & hypothetical protein & 4,31 & $6,291 \times(10)-07$ & $-1,42$ & 0,0374456 \\
\hline AT4G23810 & WRKY family transcription factor & 4,12 & $2,277 \times(10)-05$ & $-1,42$ & 0,0460651 \\
\hline AT1G66960 & Terpenoid cyclases family protein & 5,35 & $1,669 \times(10)-07$ & $-1,42$ & 0,0433531 \\
\hline AT1G12290 & $\begin{array}{l}\text { Disease resistance protein (CC-NBS- } \\
\text { LRR class) family }\end{array}$ & 2,94 & $2,051 \times(10)-05$ & $-1,41$ & 0,0149593 \\
\hline AT1G11300 & $\begin{array}{l}\text { G-type lectin S-receptor-like } \\
\text { Serine/Threonine-kinase }\end{array}$ & 1,23 & 0,0247265 & $-1,39$ & 0,0058527 \\
\hline AT3G50480 & homolog of RPW8 4 & 4,47 & $6,936 \times(10)-08$ & $-1,38$ & 0,0371792 \\
\hline AT1G14260 & $\begin{array}{l}\text { RING/FYVE/PHD zinc finger } \\
\text { superfamily protein }\end{array}$ & 2,57 & $1,116 \times(10)-06$ & $-1,38$ & 0,0016024 \\
\hline AT1G10340 & Ankyrin repeat family protein & 3,62 & $1,134 \times(10)-05$ & $-1,36$ & 0,0472626 \\
\hline AT1G13550 & hypothetical protein (DUF1262) & 3,64 & $3,546 \times(10)-13$ & $-1,36$ & 0,001282 \\
\hline AT3G61280 & $\begin{array}{l}\text { O-glucosyltransferase rumi-like } \\
\text { protein (DUF821) }\end{array}$ & 1,25 & 0,0001559 & $-1,36$ & $1,659 \times(10)-05$ \\
\hline AT1G69720 & heme oxygenase 3 & 1,63 & $6,463 \times(10)-06$ & $-1,35$ & $2,58 \times(10)-05$ \\
\hline AT1G80130 & $\begin{array}{l}\text { Tetratricopeptide repeat (TPR)-like } \\
\text { superfamily protein }\end{array}$ & 1,48 & $7,693 \times(10)-08$ & $-1,35$ & $8,052 \times(10)-07$ \\
\hline AT1G14880 & PLANT CADMIUM RESISTANCE 1 & 5,14 & $1,958 \times(10)-14$ & $-1,35$ & 0,012417 \\
\hline AT5G48570 & $\begin{array}{l}\text { FKBP-type peptidyl-prolyl cis-trans } \\
\text { isomerase family protein }\end{array}$ & 1,89 & 0,0008842 & $-1,34$ & 0,0063089 \\
\hline AT2G19190 & $\begin{array}{l}\text { FLG22-induced receptor-like kinase } \\
1\end{array}$ & 7,32 & $1,224 \times(10)-26$ & $-1,34$ & 0,0024351 \\
\hline AT2G26650 & $\mathrm{K}+$ transporter 1 & 1,12 & 0,0020268 & $-1,33$ & $6,97 \times(10)-05$ \\
\hline AT4G23220 & cysteine-rich RECEPTOR-like kinase & 1,92 & $5,026 \times(10)-06$ & $-1,33$ & 0,0011626 \\
\hline AT5G10520 & ROP binding protein kinases 1 & 1,59 & 0,0004003 & $-1,32$ & 0,0018164 \\
\hline AT2G43150 & $\begin{array}{l}\text { Proline-rich extensin-like family } \\
\text { protein }\end{array}$ & 2,04 & $7,069 \times(10)-12$ & $-1,30$ & $4,042 \times(10)-06$ \\
\hline
\end{tabular}




\begin{tabular}{|c|c|c|c|c|c|}
\hline AT4G39830 & Cupredoxin superfamily protein & 4,19 & $2,43 \times(10)-16$ & $-1,30$ & 0,0019542 \\
\hline AT5G02490 & $\begin{array}{l}\text { Heat shock protein } 70 \text { (Hsp 70) } \\
\text { family protein }\end{array}$ & 4,04 & $1,631 \times(10)-06$ & $-1,29$ & 0,0462019 \\
\hline AT2G29720 & $\begin{array}{l}\text { FAD/NAD(P)-binding } \\
\text { oxidoreductase family protein }\end{array}$ & 2,93 & $3,191 \times(10)-08$ & $-1,28$ & 0,0031046 \\
\hline AT4G37290 & transmembrane protein & 2,46 & 0,0020341 & $-1,28$ & 0,0309924 \\
\hline AT1G80120 & LURP-one-like protein (DUF567) & 1,24 & 0,0268152 & $-1,28$ & 0,0074919 \\
\hline AT5G47960 & RAB GTPase homolog A4C & 2,20 & 0,0039651 & $-1,28$ & 0,0359747 \\
\hline AT3G02610 & $\begin{array}{l}\text { plant stearoyl-acyl-carrier } \\
\text { desaturase family protein }\end{array}$ & 1,21 & 0,0064512 & $-1,28$ & 0,0025592 \\
\hline AT5G40240 & $\begin{array}{l}\text { nodulin MtN21 /EamA-like } \\
\text { transporter family protein }\end{array}$ & 1,08 & 0,0002874 & $-1,26$ & $1,404 \times(10)-05$ \\
\hline AT1G67360 & $\begin{array}{l}\text { Rubber elongation factor protein } \\
\text { (REF) }\end{array}$ & 2,10 & 0,0011445 & $-1,26$ & 0,0278485 \\
\hline AT3G25020 & receptor like protein 42 & 2,15 & $3,135 \times(10)-09$ & $-1,24$ & 0,000686 \\
\hline AT5G45380 & $\begin{array}{l}\text { urea-proton symporter } \\
\text { DEGRADATION OF UREA } 3 \text { (DUR3) }\end{array}$ & 3,81 & $5,729 \times(10)-18$ & $-1,23$ & 0,0004854 \\
\hline AT4G21903 & MATE efflux family protein & 3,38 & $3,698 \times(10)-11$ & $-1,23$ & 0,0038259 \\
\hline AT3G53235 & hypothetical protein & 1,65 & 0,0002818 & $-1,23$ & 0,0116453 \\
\hline AT2G21550 & $\begin{array}{l}\text { Bifunctional dihydrofolate } \\
\text { reductase/thymidylate synthase }\end{array}$ & 1,18 & 0,0068213 & $-1,22$ & 0,0024884 \\
\hline AT1G17147 & VQ motif-containing protein & 1,54 & 0,0151508 & $-1,21$ & 0,0456248 \\
\hline AT5G05320 & $\begin{array}{l}\text { FAD/NAD(P)-binding } \\
\text { oxidoreductase family protein }\end{array}$ & 1,66 & $2,407 \times(10)-05$ & $-1,21$ & 0,0005402 \\
\hline AT2G46150 & $\begin{array}{l}\text { Late embryogenesis abundant } \\
\text { (LEA) hydroxyproline-rich } \\
\text { glycoprotein family }\end{array}$ & 3,03 & $2,129 \times(10)-07$ & $-1,21$ & 0,0222347 \\
\hline AT1G18570 & myb domain protein 51 & 2,57 & $4,738 \times(10)-11$ & $-1,20$ & 0,0002996 \\
\hline AT1G65486 & transmembrane protein & 1,65 & $1,855 \times(10)-07$ & $-1,20$ & 0,0003927 \\
\hline AT5G45440 & $\begin{array}{l}\text { P-loop containing nucleoside } \\
\text { triphosphate hydrolases } \\
\text { superfamily protein }\end{array}$ & 3,06 & $7,398 \times(10)-11$ & $-1,18$ & 0,0027834 \\
\hline AT3G05650 & receptor like protein 32 & 1,16 & $1,837 \times(10)-05$ & $-1,18$ & 0,0001371 \\
\hline AT4G23230 & cysteine-rich RECEPTOR-like kinase & 1,98 & $2,129 \times(10)-05$ & $-1,17$ & 0,0076209 \\
\hline AT3G52400 & syntaxin of plants 122 & 3,05 & $4,42 \times(10)-06$ & $-1,17$ & 0,0270814 \\
\hline AT4G27480 & $\begin{array}{l}\text { Core-2/I-branching beta-1\%2C6-N- } \\
\text { acetylglucosaminyltransferase } \\
\text { family protein }\end{array}$ & 1,31 & 0,0007331 & $-1,17$ & 0,0020566 \\
\hline AT3G52430 & $\begin{array}{l}\text { alpha/beta-Hydrolases superfamily } \\
\text { protein }\end{array}$ & 2,21 & $5,083 \times(10)-05$ & $-1,16$ & 0,0189494 \\
\hline AT3G26500 & $\begin{array}{l}\text { plant intracellular ras group-related } \\
\text { LRR } 2\end{array}$ & 4,05 & $4,054 \times(10)-09$ & $-1,16$ & 0,0245337 \\
\hline AT4G26120 & $\begin{array}{l}\text { Ankyrin repeat family protein / } \\
\text { BTB/POZ domain-containing } \\
\text { protein }\end{array}$ & 3,15 & $1,036 \times(10)-09$ & $-1,15$ & 0,0048892 \\
\hline AT5G58940 & $\begin{array}{l}\text { calmodulin-binding receptor-like } \\
\text { cytoplasmic kinase } 1\end{array}$ & 2,89 & $8,155 \times(10)-10$ & $-1,15$ & 0,0029082 \\
\hline AT4G14390 & Ankyrin repeat family protein & 4,89 & $2,812 \times(10)-19$ & $-1,15$ & 0,0054623 \\
\hline AT3G23230 & $\begin{array}{l}\text { Integrase-type DNA-binding } \\
\text { superfamily protein }\end{array}$ & 2,20 & 0,0001706 & $-1,14$ & 0,031412 \\
\hline AT5G65090 & DNAse I-like superfamily protein & 3,43 & $4,697 \times(10)-07$ & $-1,14$ & 0,0448194 \\
\hline AT5G22250 & $\begin{array}{l}\text { Polynucleotidyl transferase } \% 2 \mathrm{C} \\
\text { ribonuclease } \mathrm{H} \text {-like superfamily } \\
\text { protein }\end{array}$ & 1,50 & 0,000873 & $-1,14$ & 0,0032321 \\
\hline
\end{tabular}




\begin{tabular}{|c|c|c|c|c|c|}
\hline AT5G60800 & $\begin{array}{l}\text { Heavy metal } \\
\text { transport/detoxification } \\
\text { superfamily protein }\end{array}$ & 2,14 & $3,867 \times(10)-06$ & $-1,13$ & 0,0042004 \\
\hline AT5G64530 & xylem NAC domain 1 & 1,13 & 0,0077468 & $-1,12$ & 0,0100867 \\
\hline AT4G30230 & hypothetical protein & 1,60 & $8,75 \times(10)-07$ & $-1,11$ & 0,0002783 \\
\hline AT3G25510 & $\begin{array}{l}\text { disease resistance protein (TIR- } \\
\text { NBS-LRR class) family protein }\end{array}$ & 2,92 & $2,438 \times(10)-05$ & $-1,11$ & 0,0367384 \\
\hline AT5G67450 & zinc-finger protein 1 & 2,95 & $2,227 \times(10)-09$ & $-1,10$ & 0,0169416 \\
\hline AT4G24570 & dicarboxylate carrier 2 & 2,56 & 0,000151 & $-1,10$ & 0,0402864 \\
\hline AT2G38860 & $\begin{array}{l}\text { Class I glutamine amidotransferase- } \\
\text { like superfamily protein }\end{array}$ & 2,34 & $5,681 \times(10)-13$ & $-1,09$ & $7,486 \times(10)-05$ \\
\hline AT1G08450 & calreticulin 3 & 2,55 & $6,121 \times(10)-11$ & $-1,09$ & 0,0014734 \\
\hline AT3G55950 & CRINKLY4 related 3 & 2,38 & $1,769 \times(10)-13$ & $-1,09$ & $4,973 \times(10)-05$ \\
\hline AT3G54640 & tryptophan synthase alpha chain & 1,68 & $1,155 \times(10)-08$ & $-1,09$ & $2,633 \times(10)-05$ \\
\hline AT1G22180 & $\begin{array}{l}\text { Sec14p-like phosphatidylinositol } \\
\text { transfer family protein }\end{array}$ & 1,37 & 0,0001901 & $-1,08$ & 0,00095 \\
\hline AT5G27030 & TOPLESS-related 3 & 1,75 & $3,694 \times(10)-09$ & $-1,07$ & $6,321 \times(10)-05$ \\
\hline AT1G28370 & ERF domain protein 11 & 2,76 & $1,393 \times(10)-09$ & $-1,07$ & 0,0035271 \\
\hline AT2G18000 & TBP-associated factor 14 & 3,28 & $1,107 \times(10)-09$ & $-1,07$ & 0,0109719 \\
\hline AT5G53370 & $\begin{array}{l}\text { pectin methylesterase PCR } \\
\text { fragment } \mathrm{F}\end{array}$ & 2,23 & $4,114 \times(10)-14$ & $-1,06$ & 0,0001657 \\
\hline AT3G48090 & $\begin{array}{l}\text { alpha/beta-Hydrolases superfamily } \\
\text { protein }\end{array}$ & 1,36 & 0,0013034 & $-1,06$ & 0,0136647 \\
\hline AT1G25220 & $\begin{array}{l}\text { anthranilate synthase beta subunit } \\
1\end{array}$ & 1,81 & $9,472 \times(10)-06$ & $-1,06$ & 0,0026402 \\
\hline AT3G06755 & & 1,49 & 0,0005365 & $-1,05$ & 0,007607 \\
\hline AT1G21460 & Nodulin MtN3 family protein & 1,04 & 0,0014404 & $-1,05$ & 0,0002126 \\
\hline AT1G20060 & $\begin{array}{l}\text { ATP binding microtubule motor } \\
\text { family protein }\end{array}$ & 1,33 & 0,0006228 & $-1,04$ & 0,0104154 \\
\hline AT3G28930 & $\begin{array}{l}\text { AIG2-like (avirulence induced gene) } \\
\text { family protein }\end{array}$ & 2,14 & $2,481 \times(10)-06$ & $-1,03$ & 0,0062296 \\
\hline AT1G33840 & LURP-one-like protein (DUF567) & 4,57 & $2,244 \times(10)-10$ & $-1,03$ & 0,0272209 \\
\hline AT5G54490 & pinoid-binding protein 1 & 2,65 & $5,27 \times(10)-06$ & $-1,03$ & 0,0399328 \\
\hline AT2G47130 & $\begin{array}{l}\text { NAD(P)-binding Rossmann-fold } \\
\text { superfamily protein }\end{array}$ & 3,72 & $1,449 \times(10)-12$ & $-1,03$ & 0,0250235 \\
\hline AT5G64870 & $\begin{array}{l}\text { SPFH/Band 7/PHB domain- } \\
\text { containing membrane-associated } \\
\text { protein family }\end{array}$ & 1,19 & 0,009279 & $-1,02$ & 0,0228105 \\
\hline AT2G32160 & $\begin{array}{l}\text { S-adenosyl-L-methionine- } \\
\text { dependent methyltransferases } \\
\text { superfamily protein }\end{array}$ & 1,69 & $2,754 \times(10)-09$ & $-1,02$ & 0,0002036 \\
\hline AT5G38340 & $\begin{array}{l}\text { Disease resistance protein (TIR- } \\
\text { NBS-LRR class) family }\end{array}$ & 1,52 & 0,0138478 & $-1,02$ & 0,025269 \\
\hline AT2G41100 & $\begin{array}{l}\text { Calcium-binding EF hand family } \\
\text { protein }\end{array}$ & 2,74 & $2,302 \times(10)-07$ & $-1,02$ & 0,0187996 \\
\hline AT1G79400 & cation/H+ exchanger 2 & 5,64 & $3,164 \times(10)-20$ & $-1,02$ & 0,0180847 \\
\hline AT5G54710 & Ankyrin repeat family protein & 2,27 & $2,54 \times(10)-13$ & $-1,02$ & 0,0001646 \\
\hline AT4G31230 & $\begin{array}{l}\text { kinase with adenine nucleotide } \\
\text { alpha hydrolases-like domain- } \\
\text { containing protein }\end{array}$ & 2,22 & $4,104 \times(10)-06$ & $-1,02$ & 0,0133015 \\
\hline AT3G52460 & $\begin{array}{l}\text { hydroxyproline-rich glycoprotein } \\
\text { family protein }\end{array}$ & 2,60 & $5,779 \times(10)-11$ & $-1,01$ & 0,0022338 \\
\hline AT2G31880 & $\begin{array}{l}\text { Leucine-rich repeat protein kinase } \\
\text { family protein }\end{array}$ & 1,69 & 0,0001615 & $-1,01$ & 0,0188305 \\
\hline
\end{tabular}




\begin{tabular}{l|l|r|r||r|r}
\hline AT4G21940 & $\begin{array}{l}\text { calcium-dependent protein kinase } \\
\text { 15 }\end{array}$ & 1,16 & 0,0256103 & $-1,00$ & 0,0419332 \\
\hline AT3G20960 & $\begin{array}{l}\text { cytochrome P450\%2C family } \\
\text { 705\%2C subfamily A\%2C } \\
\text { polypeptide 33 }\end{array}$ & 1,09 & 0,0060072 & $-1,00$ & 0,0057996 \\
\hline AT4G23030 & MATE efflux family protein & 2,02 & $1,49 \times(10)-05$ & $-1,00$ & 0,0166317 \\
\hline AT1G29240 & $\begin{array}{l}\text { transcription initiation factor TFIID } \\
\text { subunit\%2C putative (DUF688) }\end{array}$ & 1,46 & $4,262 \times(10)-07$ & $-1,00$ & 0,0003396 \\
\hline AT3G04720 & pathogenesis-related 4 & 2,17 & $3,417 \times(10)-05$ & $-1,00$ & 0,0182848 \\
\hline AT4G18630 & hypothetical protein (DUF688) & 1,38 & $7,325 \times(10)-05$ & $-1,00$ & 0,0030807
\end{tabular}

Supplementary Table 2239 genes which are SA-inducible in sid 2 and less expressed in sid $2 \operatorname{tga} 1$ tga 4 after SA treatment after twenty-four hours.

\begin{tabular}{|c|c|c|c|c|c|}
\hline \multicolumn{2}{|c|}{ Identification } & \multicolumn{2}{|c|}{ SA induction in sid2 (24h) } & \multicolumn{2}{|c|}{$\begin{array}{l}\text { sid2 tga1 tga4 vs sid2 (SA- } \\
\text { 24h) }\end{array}$} \\
\hline AGI code & Note & $\log _{2} \mathrm{FC}$ & AGI code & Note & $\log _{2} \mathrm{FC}$ \\
\hline AT4G21840 & methionine sulfoxide reductase $B 8$ & 8,46 & 0,0047453 & $-7,45$ & 0,0096877 \\
\hline AT1G71390 & receptor like protein 11 & 7,09 & 0,0108189 & $-7,17$ & 0,0108974 \\
\hline AT5G63225 & $\begin{array}{l}\text { Carbohydrate-binding X8 domain } \\
\text { superfamily protein }\end{array}$ & 5,80 & 0,0401604 & $-6,56$ & 0,0218432 \\
\hline AT3G57260 & beta-1\%2C3-glucanase 2 & 6,49 & 0,0072196 & $-6,26$ & 0,0092069 \\
\hline AT4G04500 & $\begin{array}{l}\text { cysteine-rich RLK (RECEPTOR-like } \\
\text { protein kinase) } 37\end{array}$ & 6,24 & 0,0190337 & $-6,17$ & 0,0199461 \\
\hline AT1G33950 & $\begin{array}{l}\text { Avirulence induced gene (AIG1) } \\
\text { family protein }\end{array}$ & 7,34 & 0,0014564 & $-6,11$ & 0,004351 \\
\hline AT1G09080 & $\begin{array}{l}\text { Heat shock protein } 70 \text { ( Hsp 70) } \\
\text { family protein }\end{array}$ & 5,76 & 0,0261898 & $-5,73$ & 0,0276625 \\
\hline AT3G49340 & $\begin{array}{l}\text { Cysteine proteinases superfamily } \\
\text { protein }\end{array}$ & 4,71 & 0,0302368 & $-5,22$ & 0,0213777 \\
\hline AT4G36430 & Peroxidase superfamily protein & 4,54 & 0,0108028 & $-4,98$ & 0,0055653 \\
\hline AT3G45330 & $\begin{array}{l}\text { Concanavalin A-like lectin protein } \\
\text { kinase family protein }\end{array}$ & 6,01 & 0,0019067 & $-4,78$ & 0,0060355 \\
\hline AT4G17660 & Protein kinase superfamily protein & 4,86 & 0,0296364 & $-4,73$ & 0,0343175 \\
\hline AT4G39020 & SH3 domain-containing protein & 4,19 & 0,0282675 & $-4,72$ & 0,019904 \\
\hline AT5G66890 & $\begin{array}{l}\text { Leucine-rich repeat (LRR) family } \\
\text { protein }\end{array}$ & 4,29 & 0,0300554 & $-4,56$ & 0,0239332 \\
\hline AT4G23150 & $\begin{array}{l}\text { cysteine-rich RLK (RECEPTOR-like } \\
\text { protein kinase) } 7\end{array}$ & 4,73 & 0,0009428 & $-4,55$ & 0,0014025 \\
\hline AT3G44350 & NAC domain containing protein 61 & 5,05 & 0,0103461 & $-4,34$ & 0,026985 \\
\hline AT4G23310 & $\begin{array}{l}\text { cysteine-rich RLK (RECEPTOR-like } \\
\text { protein kinase) } 23\end{array}$ & 5,11 & 0,0083659 & $-4,28$ & 0,0262395 \\
\hline AT5G45090 & phloem protein 2-A7 & 4,47 & 0,0109615 & $-4,26$ & 0,0153471 \\
\hline AT4G21850 & methionine sulfoxide reductase B9 & 4,71 & 0,0008276 & $-4,20$ & 0,0026934 \\
\hline AT1G75040 & pathogenesis-related protein 5 & 5,09 & 0,0001463 & $-4,20$ & 0,0016724 \\
\hline AT4G10500 & $\begin{array}{l}\text { 2-oxoglutarate (2OG) and Fe(II)- } \\
\text { dependent oxygenase superfamily } \\
\text { protein }\end{array}$ & 6,10 & $4,083 \times(10)-05$ & $-4,10$ & 0,0032648 \\
\hline AT4G15150 & glycine-rich protein & 3,56 & 0,0055515 & $-4,09$ & 0,0028348 \\
\hline AT3G15536 & other_RNA & 5,35 & 0,0080679 & $-4,07$ & 0,0343876 \\
\hline AT4G04510 & $\begin{array}{l}\text { cysteine-rich RLK (RECEPTOR-like } \\
\text { protein kinase) } 38\end{array}$ & 4,71 & 0,0085636 & $-4,03$ & 0,0243258 \\
\hline AT1G58225 & hypothetical protein & 3,87 & 0,0445509 & $-3,98$ & 0,0372764 \\
\hline
\end{tabular}




\begin{tabular}{|c|c|c|c|c|c|}
\hline AT4G17670 & $\begin{array}{l}\text { senescence-associated family } \\
\text { protein (DUF581) }\end{array}$ & 3,25 & 0,0017219 & $-3,98$ & 0,0001694 \\
\hline AT5G55410 & $\begin{array}{l}\text { Bifunctional inhibitor/lipid-transfer } \\
\text { protein/seed storage } 2 \mathrm{~S} \text { albumin } \\
\text { superfamily protein }\end{array}$ & 5,96 & $2,181 \times(10)-06$ & $-3,97$ & $9,453 \times(10)-05$ \\
\hline AT3G57950 & cotton fiber protein & 5,46 & 0,013328 & $-3,95$ & 0,0492141 \\
\hline AT3G24900 & receptor like protein 39 & 5,71 & $8,101 \times(10)-05$ & $-3,74$ & 0,0068191 \\
\hline AT4G05030 & Copper transport protein family & 5,38 & 0,0064951 & $-3,73$ & 0,0294175 \\
\hline AT1G33960 & $\begin{array}{l}\text { P-loop containing nucleoside } \\
\text { triphosphate hydrolases } \\
\text { superfamily protein }\end{array}$ & 6,64 & $8,132 \times(10)-05$ & $-3,54$ & 0,0179227 \\
\hline AT1G01680 & plant U-box 54 & 4,75 & 0,002389 & $-3,52$ & 0,0191081 \\
\hline AT3G25010 & receptor like protein 41 & 4,89 & 0,0002237 & $-3,43$ & 0,007257 \\
\hline AT2G18660 & plant natriuretic peptide $A$ & 5,54 & 0,0002607 & $-3,39$ & 0,0169427 \\
\hline AT5G55460 & $\begin{array}{l}\text { Bifunctional inhibitor/lipid-transfer } \\
\text { protein/seed storage } 2 \mathrm{~S} \text { albumin } \\
\text { superfamily protein }\end{array}$ & 4,27 & $1,677 \times(10)-05$ & $-3,39$ & 0,0005828 \\
\hline AT1G04600 & myosin XI A & 3,18 & 0,0298744 & $-3,38$ & 0,0237923 \\
\hline AT3G06890 & transmembrane protein & 2,46 & 0,0075998 & $-3,38$ & 0,000617 \\
\hline AT5G40010 & AAA-ATPase 1 & 6,70 & 0,0003033 & $-3,36$ & 0,0324549 \\
\hline AT1G57560 & myb domain protein 50 & 3,12 & 0,0008809 & $-3,34$ & 0,0005515 \\
\hline AT4G01130 & $\begin{array}{l}\text { GDSL-like Lipase/Acylhydrolase } \\
\text { superfamily protein }\end{array}$ & 1,50 & $2,6 \times(10)-05$ & $-3,33$ & $5,023 \times(10)-17$ \\
\hline AT5G18470 & $\begin{array}{l}\text { Curculin-like (mannose-binding) } \\
\text { lectin family protein }\end{array}$ & 3,55 & $5,903 \times(10)-05$ & $-3,32$ & 0,0001547 \\
\hline AT5G26220 & ChaC-like family protein & 3,85 & 0,0238815 & $-3,31$ & 0,047149 \\
\hline AT2G43580 & Chitinase family protein & 6,18 & 0,0002629 & $-3,28$ & 0,0145103 \\
\hline AT2G13810 & $\begin{array}{l}\text { AGD2-like defense response } \\
\text { protein } 1\end{array}$ & 6,03 & $3,93 \times(10)-05$ & $-3,27$ & 0,0116075 \\
\hline AT3G13640 & RNAse I inhibitor protein 1 & 2,73 & 0,0038735 & $-3,19$ & 0,0011035 \\
\hline AT4G23140 & $\begin{array}{l}\text { cysteine-rich RLK (RECEPTOR-like } \\
\text { protein kinase) } 6\end{array}$ & 3,75 & $6,528 \times(10)-06$ & $-3,19$ & 0,0001372 \\
\hline AT3G13610 & $\begin{array}{l}\text { 2-oxoglutarate (2OG) and Fe(II)- } \\
\text { dependent oxygenase superfamily } \\
\text { protein }\end{array}$ & 6,29 & $2,814 \times(10)-08$ & $-3,17$ & 0,0015059 \\
\hline AT4G23160 & cysteine-rich RECEPTOR-like kinase & 3,63 & $1,984 \times(10)-05$ & $-3,15$ & 0,0001206 \\
\hline AT5G57010 & calmodulin-binding family protein & 3,91 & 0,0070255 & $-3,13$ & 0,0298466 \\
\hline AT3G46520 & actin-12 & 3,62 & 0,0084914 & $-3,12$ & 0,0156111 \\
\hline AT1G09932 & $\begin{array}{l}\text { Phosphoglycerate mutase family } \\
\text { protein }\end{array}$ & 4,12 & 0,0057383 & $-3,11$ & 0,0302517 \\
\hline AT4G39030 & MATE efflux family protein & 4,00 & 0,0001764 & $-3,07$ & 0,0031978 \\
\hline AT1G53100 & $\begin{array}{l}\text { Core-2/I-branching beta-1\%2C6-N- } \\
\text { acetylglucosaminyltransferase } \\
\text { family protein }\end{array}$ & 7,42 & $3,053 \times(10)-09$ & $-3,07$ & 0,0009374 \\
\hline AT2G32680 & receptor like protein 23 & 4,15 & $2,646 \times(10)-06$ & $-3,06$ & 0,0004017 \\
\hline AT4G04540 & $\begin{array}{l}\text { cysteine-rich RLK (RECEPTOR-like } \\
\text { protein kinase) } 39\end{array}$ & 3,82 & $7,433 \times(10)-06$ & $-3,04$ & 0,0001887 \\
\hline AT1G66960 & Terpenoid cyclases family protein & 6,14 & $3,476 \times(10)-11$ & $-3,03$ & $7,227 \times(10)-05$ \\
\hline AT4G16260 & $\begin{array}{l}\text { Glycosyl hydrolase superfamily } \\
\text { protein }\end{array}$ & 3,91 & 0,0108409 & $-3,00$ & 0,040848 \\
\hline AT4G30430 & tetraspanin9 & 5,12 & $1,236 \times(10)-05$ & $-2,98$ & 0,0028582 \\
\hline AT2G43000 & NAC domain containing protein 42 & 4,67 & $1,231 \times(10)-07$ & $-2,98$ & 0,0002824 \\
\hline
\end{tabular}




\begin{tabular}{|c|c|c|c|c|c|}
\hline AT1G76040 & $\begin{array}{l}\text { calcium-dependent protein kinase } \\
29\end{array}$ & 2,97 & 0,0001039 & $-2,96$ & 0,0001235 \\
\hline AT1G21240 & wall associated kinase 3 & 5,47 & 0,0004513 & $-2,95$ & 0,038816 \\
\hline AT5G10760 & $\begin{array}{l}\text { Eukaryotic aspartyl protease family } \\
\text { protein }\end{array}$ & 4,02 & $8,243 \times(10)-16$ & $-2,91$ & $2,856 \times(10)-09$ \\
\hline AT3G10590 & $\begin{array}{l}\text { Duplicated homeodomain-like } \\
\text { superfamily protein }\end{array}$ & 2,11 & 0,0171256 & $-2,87$ & 0,0027154 \\
\hline AT5G08240 & transmembrane protein & 5,49 & $2,957 \times(10)-06$ & $-2,84$ & 0,0078885 \\
\hline AT5G39670 & $\begin{array}{l}\text { Calcium-binding EF-hand family } \\
\text { protein }\end{array}$ & 4,34 & 0,0020225 & $-2,83$ & 0,0342107 \\
\hline AT3G13100 & $\begin{array}{l}\text { multidrug resistance-associated } \\
\text { protein } 7\end{array}$ & 3,67 & 0,0015076 & $-2,82$ & 0,0127447 \\
\hline AT4G28790 & $\begin{array}{l}\text { basic helix-loop-helix (bHLH) DNA- } \\
\text { binding superfamily protein }\end{array}$ & 2,52 & 0,0039153 & $-2,77$ & 0,0016224 \\
\hline AT5G09175 & $\begin{array}{l}\text { Natural antisense transcript } \\
\text { overlaps with AT5G64000 }\end{array}$ & 5,45 & 0,0012862 & $-2,76$ & 0,0355706 \\
\hline AT3G01175 & transmembrane protein & 2,93 & 0,041433 & $-2,74$ & 0,0440835 \\
\hline AT3G45410 & $\begin{array}{l}\text { Concanavalin A-like lectin protein } \\
\text { kinase family protein }\end{array}$ & 2,19 & 0,02732 & $-2,73$ & 0,0062917 \\
\hline AT4G23200 & $\begin{array}{l}\text { cysteine-rich RLK (RECEPTOR-like } \\
\text { protein kinase) } 12\end{array}$ & 2,95 & 0,0002084 & $-2,71$ & 0,0006703 \\
\hline AT3G17690 & cyclic nucleotide gated channel 19 & 4,09 & $7,092 \times(10)-05$ & $-2,68$ & 0,0072361 \\
\hline AT5G02220 & cyclin-dependent kinase inhibitor & 3,23 & 0,0003606 & $-2,66$ & 0,0039943 \\
\hline AT1G76955 & Expressed protein & 2,19 & $3,218 \times(10)-09$ & $-2,64$ & $6,463 \times(10)-13$ \\
\hline AT1G58420 & $\begin{array}{l}\text { Uncharacterized conserved protein } \\
\text { UCP031279 }\end{array}$ & 2,61 & 0,0102589 & $-2,61$ & 0,0123598 \\
\hline AT3G55700 & $\begin{array}{l}\text { UDP-Glycosyltransferase } \\
\text { superfamily protein }\end{array}$ & 3,46 & 0,0026046 & $-2,55$ & 0,0248289 \\
\hline AT4G38560 & $\begin{array}{l}\text { phospholipase-like protein (PEARLI } \\
\text { 4) family protein }\end{array}$ & 3,46 & 0,0025213 & $-2,55$ & 0,0240562 \\
\hline AT3G60540 & $\begin{array}{l}\text { Preprotein translocase Sec\%2C } \\
\text { Sec61-beta subunit protein }\end{array}$ & 3,37 & $4,791 \times(10)-05$ & $-2,55$ & 0,0021708 \\
\hline AT3G26470 & $\begin{array}{l}\text { Powdery mildew resistance } \\
\text { protein } \% 2 C \text { RPW8 domain- } \\
\text { containing protein }\end{array}$ & 3,99 & 0,0002534 & $-2,54$ & 0,0153138 \\
\hline AT5G11920 & 6-\%261-fructan exohydrolase & 5,73 & $1,444 \times(10)-11$ & $-2,53$ & 0,0005445 \\
\hline AT5G60760 & $\begin{array}{l}\text { P-loop containing nucleoside } \\
\text { triphosphate hydrolases } \\
\text { superfamily protein }\end{array}$ & 1,43 & 0,0426681 & $-2,52$ & 0,0012217 \\
\hline AT4G23210 & $\begin{array}{l}\text { cysteine-rich RLK (RECEPTOR-like } \\
\text { protein kinase) } 13\end{array}$ & 4,68 & $6,725 \times(10)-07$ & $-2,51$ & 0,0045132 \\
\hline AT1G19340 & $\begin{array}{l}\text { Methyltransferase MT-A70 family } \\
\text { protein }\end{array}$ & 1,10 & 0,0091031 & $-2,51$ & $5,073 \times(10)-08$ \\
\hline AT5G22520 & hypothetical protein & 3,93 & 0,0001074 & $-2,51$ & 0,0101017 \\
\hline AT4G03450 & Ankyrin repeat family protein & 3,66 & $1,144 \times(10)-07$ & $-2,46$ & 0,0002906 \\
\hline AT1G76960 & transmembrane protein & 4,47 & $2,03 \times(10)-08$ & $-2,45$ & 0,0011298 \\
\hline AT3G28580 & $\begin{array}{l}\text { P-loop containing nucleoside } \\
\text { triphosphate hydrolases } \\
\text { superfamily protein }\end{array}$ & 3,66 & $2,909 \times(10)-05$ & $-2,41$ & 0,0058072 \\
\hline AT5G66640 & DA1-related protein 3 & 2,49 & 0,0123137 & $-2,38$ & 0,0162303 \\
\hline AT1G35230 & arabinogalactan protein 5 & 5,15 & $1,099 \times(10)-05$ & $-2,35$ & 0,036132 \\
\hline AT1G21310 & extensin 3 & 3,88 & $1,142 \times(10)-11$ & $-2,33$ & $2,349 \times(10)-05$ \\
\hline AT1G68200 & $\begin{array}{l}\text { Zinc finger C-x8-C-x5-C-x3-H type } \\
\text { family protein }\end{array}$ & 2,04 & 0,0114954 & $-2,33$ & 0,0053719 \\
\hline AT5G64510 & tunicamycin induced protein & 3,13 & $1,287 \times(10)-05$ & $-2,33$ & 0,0011459 \\
\hline
\end{tabular}




\begin{tabular}{|c|c|c|c|c|c|}
\hline AT1G14260 & $\begin{array}{l}\text { RING/FYVE/PHD zinc finger } \\
\text { superfamily protein }\end{array}$ & 2,24 & $1,89 \times(10)-05$ & $-2,33$ & $3,377 \times(10)-06$ \\
\hline AT1G30040 & gibberellin 2-oxidase & 2,07 & $1,583 \times(10)-05$ & $-2,32$ & $2,205 \times(10)-06$ \\
\hline AT3G45860 & $\begin{array}{l}\text { cysteine-rich RLK (RECEPTOR-like } \\
\text { protein kinase) } 4\end{array}$ & 2,00 & 0,001073 & $-2,32$ & 0,0002401 \\
\hline AT5G47850 & CRINKLY4 related 4 & 3,99 & 0,0011281 & $-2,27$ & 0,0449571 \\
\hline AT5G52740 & Copper transport protein family & 2,29 & 0,0072632 & $-2,27$ & 0,0085848 \\
\hline AT5G10380 & RING/U-box superfamily protein & 2,84 & 0,0005037 & $-2,27$ & 0,0049622 \\
\hline AT5G37600 & hypothetical protein & 3,63 & $2,67 \times(10)-21$ & $-2,25$ & $2,599 \times(10)-09$ \\
\hline AT4G18253 & $\begin{array}{l}\text { receptor Serine/Threonine kinase- } \\
\text { like protein }\end{array}$ & 3,65 & $1,232 \times(10)-09$ & $-2,24$ & 0,000155 \\
\hline AT5G22530 & hypothetical protein & 2,91 & $1,059 \times(10)-05$ & $-2,24$ & 0,0005826 \\
\hline AT3G07520 & glutamate receptor 1.4 & 2,67 & $2,68 \times(10)-06$ & $-2,23$ & $9,026 \times(10)-05$ \\
\hline AT1G72540 & Protein kinase superfamily protein & 4,73 & $4,115 \times(10)-05$ & $-2,19$ & 0,0328851 \\
\hline AT5G43910 & $\begin{array}{l}\text { pfkB-like carbohydrate kinase } \\
\text { family protein }\end{array}$ & 3,64 & 0,000564 & $-2,18$ & 0,0372566 \\
\hline AT1G74940 & $\begin{array}{l}\text { cyclin-dependent kinase } \% 2 C \\
\text { putative (DUF581) }\end{array}$ & 1,96 & $8,881 \times(10)-08$ & $-2,16$ & $2,614 \times(10)-09$ \\
\hline AT1G66465 & transmembrane protein & 4,12 & $1,024 \times(10)-05$ & $-2,16$ & 0,0138979 \\
\hline AT5G55450 & $\begin{array}{l}\text { Bifunctional inhibitor/lipid-transfer } \\
\text { protein/seed storage } 2 \mathrm{~S} \text { albumin } \\
\text { superfamily protein }\end{array}$ & 3,04 & 0,0005445 & $-2,15$ & 0,0088262 \\
\hline AT1G68840 & related to $A B \mid 3 / V P 12$ & 1,89 & 0,0157952 & $-2,14$ & 0,0081371 \\
\hline AT5G53830 & VQ motif-containing protein & 2,39 & 0,0015019 & $-2,14$ & 0,0039333 \\
\hline AT2G17710 & Big1 & 1,75 & $4,868 \times(10)-05$ & $-2,13$ & $1,295 \times(10)-06$ \\
\hline AT4G18250 & $\begin{array}{l}\text { receptor Serine/Threonine kinase- } \\
\text { like protein }\end{array}$ & 3,20 & $5,133 \times(10)-10$ & $-2,12$ & $2,793 \times(10)-05$ \\
\hline AT1G59590 & ZCF37 & 2,23 & 0,0149707 & $-2,10$ & 0,0234709 \\
\hline AT1G61120 & terpene synthase 04 & 4,96 & $2,329 \times(10)-05$ & $-2,09$ & 0,0228356 \\
\hline AT4G30500 & transmembrane protein (DUF788) & 1,68 & 0,0001404 & $-2,08$ & $2,748 \times(10)-06$ \\
\hline AT3G21520 & $\begin{array}{l}\text { transmembrane protein\%2C } \\
\text { putative (DUF679 domain } \\
\text { membrane protein 1) }\end{array}$ & 3,96 & 0,0004796 & $-2,08$ & 0,0447334 \\
\hline AT1G65790 & receptor kinase 1 & 3,35 & $2,489 \times(10)-05$ & $-2,07$ & 0,0088421 \\
\hline AT3G52710 & hypothetical protein & 1,78 & $6,265 \times(10)-06$ & $-2,06$ & $4,03 \times(10)-07$ \\
\hline AT4G24190 & $\begin{array}{l}\text { Chaperone protein htpG family } \\
\text { protein }\end{array}$ & 2,30 & $5,285 \times(10)-09$ & $-2,05$ & $4,262 \times(10)-07$ \\
\hline AT5G14930 & senescence-associated gene 101 & 2,81 & $1,707 \times(10)-05$ & $-2,05$ & 0,0016395 \\
\hline AT4G23030 & MATE efflux family protein & 2,62 & $1,975 \times(10)-06$ & $-2,05$ & 0,0001931 \\
\hline AT3G02550 & LOB domain-containing protein 41 & 1,82 & 0,001811 & $-2,04$ & 0,0002933 \\
\hline AT5G48290 & $\begin{array}{l}\text { Heavy metal } \\
\text { transport/detoxification } \\
\text { superfamily protein }\end{array}$ & 4,96 & $6,081 \times(10)-06$ & $-2,04$ & 0,0260838 \\
\hline AT2G04430 & nudix hydrolase homolog 5 & 3,30 & 0,0001123 & $-2,03$ & 0,0186783 \\
\hline AT1G77510 & PDI-like 1-2 & 3,46 & $4,885 \times(10)-13$ & $-2,01$ & $2,875 \times(10)-05$ \\
\hline AT3G52748 & other_RNA & 4,16 & $9,612 \times(10)-05$ & $-2,01$ & 0,0403351 \\
\hline AT4G14640 & calmodulin 8 & 2,07 & 0,0177867 & $-2,00$ & 0,0268207 \\
\hline AT2G24850 & tyrosine aminotransferase 3 & 5,12 & $3,528 \times(10)-10$ & $-1,99$ & 0,0089679 \\
\hline AT5G56050 & $\begin{array}{l}\text { late embryogenesis abundant (LEA) } \\
\text { hydroxyproline-rich glycoprotein } \\
\text { family protein }\end{array}$ & 1,66 & 0,0308942 & $-1,98$ & 0,0083913 \\
\hline
\end{tabular}




\begin{tabular}{|c|c|c|c|c|c|}
\hline AT3G22400 & $\begin{array}{l}\text { PLAT/LH2 domain-containing } \\
\text { lipoxygenase family protein }\end{array}$ & 1,38 & 0,0012661 & $-1,97$ & $1,486 \times(10)-05$ \\
\hline AT3G52430 & $\begin{array}{l}\text { alpha/beta-Hydrolases superfamily } \\
\text { protein }\end{array}$ & 2,82 & $2,026 \times(10)-07$ & $-1,95$ & 0,0003818 \\
\hline AT4G30560 & cyclic nucleotide gated channel 9 & 1,45 & $8,957 \times(10)-06$ & $-1,93$ & $9,747 \times(10)-09$ \\
\hline AT3G50480 & homolog of RPW8 4 & 3,96 & $1,084 \times(10)-07$ & $-1,89$ & 0,009983 \\
\hline AT2G31865 & poly(ADP-ribose) glycohydrolase 2 & 3,91 & 0,000124 & $-1,88$ & 0,0474932 \\
\hline AT5G10570 & $\begin{array}{l}\text { basic helix-loop-helix (bHLH) DNA- } \\
\text { binding superfamily protein }\end{array}$ & 1,33 & 0,003735 & $-1,88$ & $7,685 \times(10)-05$ \\
\hline AT5G60800 & $\begin{array}{l}\text { Heavy metal } \\
\text { transport/detoxification } \\
\text { superfamily protein }\end{array}$ & 1,46 & 0,0006122 & $-1,87$ & $1,75 \times(10)-05$ \\
\hline AT1G65484 & transmembrane protein & 2,80 & 0,0003045 & $-1,87$ & 0,011258 \\
\hline AT4G28400 & $\begin{array}{l}\text { Protein phosphatase } 2 \text { C family } \\
\text { protein }\end{array}$ & 1,56 & 0,0075675 & $-1,87$ & 0,0015811 \\
\hline AT5G02490 & $\begin{array}{l}\text { Heat shock protein } 70 \text { (Hsp 70) } \\
\text { family protein }\end{array}$ & 2,84 & 0,0001423 & $-1,87$ & 0,012655 \\
\hline AT1G02920 & glutathione S-transferase 7 & 3,60 & $6,65 \times(10)-05$ & $-1,86$ & 0,0333108 \\
\hline AT1G64710 & $\begin{array}{l}\text { GroES-like zinc-binding alcohol } \\
\text { dehydrogenase family protein }\end{array}$ & 1,46 & 0,0001031 & $-1,84$ & $1,138 \times(10)-06$ \\
\hline AT1G61260 & cotton fiber (DUF761) & 1,66 & 0,0089611 & $-1,84$ & 0,003863 \\
\hline AT5G17330 & glutamate decarboxylase & 2,59 & 0,0035973 & $-1,84$ & 0,0359155 \\
\hline AT1G67810 & sulfur E2 & 3,33 & $4,742 \times(10)-05$ & $-1,83$ & 0,0295368 \\
\hline AT2G19130 & $\begin{array}{l}\text { S-locus lectin protein kinase family } \\
\text { protein }\end{array}$ & 1,66 & 0,0002964 & $-1,83$ & 0,0001034 \\
\hline AT2G32140 & transmembrane receptor & 3,59 & 0,0001777 & $-1,83$ & 0,0416053 \\
\hline AT3G62600 & DNAJ heat shock family protein & 2,28 & $1,53 \times(10)-06$ & $-1,81$ & 0,0002204 \\
\hline AT3G48090 & $\begin{array}{l}\text { alpha/beta-Hydrolases superfamily } \\
\text { protein }\end{array}$ & 2,21 & $1,328 \times(10)-06$ & $-1,80$ & 0,0001232 \\
\hline AT1G64065 & $\begin{array}{l}\text { Late embryogenesis abundant } \\
\text { (LEA) hydroxyproline-rich } \\
\text { glycoprotein family }\end{array}$ & 1,86 & 0,0054711 & $-1,78$ & 0,0081461 \\
\hline AT1G56150 & $\begin{array}{l}\text { SAUR-like auxin-responsive protein } \\
\text { family }\end{array}$ & 3,30 & $3,837 \times(10)-09$ & $-1,76$ & 0,0008837 \\
\hline AT1G30190 & cotton fiber protein & 2,02 & 0,0194912 & $-1,74$ & 0,0327113 \\
\hline AT5G35735 & Auxin-responsive family protein & 3,47 & $4,348 \times(10)-27$ & $-1,73$ & $5,317 \times(10)-08$ \\
\hline AT4G39210 & $\begin{array}{l}\text { Glucose-1-phosphate } \\
\text { adenylyltransferase family protein }\end{array}$ & 1,14 & 0,0012605 & $-1,72$ & $8,969 \times(10)-07$ \\
\hline AT3G22100 & $\begin{array}{l}\text { basic helix-loop-helix (bHLH) DNA- } \\
\text { binding superfamily protein }\end{array}$ & 1,62 & 0,0007296 & $-1,71$ & 0,0004341 \\
\hline AT4G13000 & $\begin{array}{l}\text { AGC (cAMP-dependent\%2C cGMP- } \\
\text { dependent and protein kinase C) } \\
\text { kinase family protein }\end{array}$ & 1,75 & 0,0149939 & $-1,71$ & 0,019533 \\
\hline AT3G08970 & $\begin{array}{l}\text { DNAJ heat shock } \mathrm{N} \text {-terminal } \\
\text { domain-containing protein }\end{array}$ & 2,59 & $5,37 \times(10)-05$ & $-1,71$ & 0,008612 \\
\hline AT1G02930 & glutathione S-transferase 6 & 4,22 & $6,586 \times(10)-07$ & $-1,70$ & 0,0367988 \\
\hline AT5G65210 & $\begin{array}{l}\text { bZIP transcription factor family } \\
\text { protein }\end{array}$ & 1,60 & $2,003 \times(10)-06$ & $-1,70$ & $2,615 \times(10)-07$ \\
\hline AT4G26120 & $\begin{array}{l}\text { Ankyrin repeat family protein / } \\
\text { BTB/POZ domain-containing } \\
\text { protein }\end{array}$ & 1,69 & 0,0003066 & $-1,70$ & 0,0002693 \\
\hline AT2G04450 & nudix hydrolase homolog 6 & 4,39 & $3,067 \times(10)-07$ & $-1,68$ & 0,043627 \\
\hline AT4G14365 & hypothetical protein & 3,38 & $1,874 \times(10)-05$ & $-1,68$ & 0,0319825 \\
\hline AT3G54960 & PDI-like 1-3 & 2,58 & $2,062 \times(10)-13$ & $-1,67$ & $4,418 \times(10)-06$ \\
\hline
\end{tabular}




\begin{tabular}{|c|c|c|c|c|c|}
\hline AT5G17760 & $\begin{array}{l}\text { P-loop containing nucleoside } \\
\text { triphosphate hydrolases } \\
\text { superfamily protein }\end{array}$ & 3,19 & $1,668 \times(10)-07$ & $-1,61$ & 0,008245 \\
\hline AT4G23885 & hypothetical protein & 2,30 & 0,0001549 & $-1,58$ & 0,008991 \\
\hline AT3G11010 & receptor like protein 34 & 3,54 & $2,025 \times(10)-09$ & $-1,58$ & 0,0079541 \\
\hline AT3G28890 & receptor like protein 43 & 3,12 & $7,492 \times(10)-09$ & $-1,57$ & 0,0036796 \\
\hline AT1G21750 & PDI-like 1-1 & 3,39 & $4,547 \times(10)-19$ & $-1,57$ & $5,328 \times(10)-05$ \\
\hline AT4G29520 & nucleophosmin & 2,47 & $1,917 \times(10)-09$ & $-1,57$ & 0,0001565 \\
\hline AT3G03640 & beta glucosidase 25 & 3,21 & $5,494 \times(10)-05$ & $-1,57$ & 0,0422562 \\
\hline AT3G23120 & receptor like protein 38 & 2,64 & $1,411 \times(10)-05$ & $-1,56$ & 0,0130367 \\
\hline AT3G03870 & transmembrane protein & 1,36 & 0,0112841 & $-1,56$ & 0,0030765 \\
\hline AT5G54490 & pinoid-binding protein 1 & 2,47 & $1,861 \times(10)-06$ & $-1,54$ & 0,0014014 \\
\hline AT1G13470 & hypothetical protein (DUF1262) & 4,19 & $3,777 \times(10)-14$ & $-1,52$ & 0,0052457 \\
\hline AT1G36370 & serine hydroxymethyltransferase 7 & 1,74 & 0,0223694 & $-1,51$ & 0,0454353 \\
\hline AT4G04570 & $\begin{array}{l}\text { cysteine-rich RLK (RECEPTOR-like } \\
\text { protein kinase) } 40\end{array}$ & 1,19 & 0,0001616 & $-1,51$ & $4,032 \times(10)-06$ \\
\hline AT3G51980 & ARM repeat superfamily protein & 1,27 & 0,0004974 & $-1,50$ & $5,198 \times(10)-05$ \\
\hline AT2G41100 & $\begin{array}{l}\text { Calcium-binding EF hand family } \\
\text { protein }\end{array}$ & 1,97 & $4,035 \times(10)-05$ & $-1,48$ & 0,0026776 \\
\hline AT5G27420 & carbon/nitrogen insensitive 1 & 2,27 & 0,0017335 & $-1,48$ & 0,0447733 \\
\hline AT2G29090 & $\begin{array}{l}\text { cytochrome } \mathrm{P} 450 \% 2 \mathrm{C} \text { family } \\
707 \% 2 \mathrm{C} \text { subfamily } \mathrm{A} \% 2 \mathrm{C} \\
\text { polypeptide } 2\end{array}$ & 2,38 & $1,443 \times(10)-06$ & $-1,47$ & 0,0017559 \\
\hline AT5G61430 & $\begin{array}{l}\text { NAC domain containing protein } \\
100\end{array}$ & 2,25 & $1,617 \times(10)-05$ & $-1,47$ & 0,0020215 \\
\hline AT1G35210 & hypothetical protein & 2,16 & 0,0007401 & $-1,44$ & 0,0212354 \\
\hline AT5G44390 & $\begin{array}{l}\text { FAD-binding Berberine family } \\
\text { protein }\end{array}$ & 1,49 & $1,121 \times(10)-06$ & $-1,43$ & $5,709 \times(10)-06$ \\
\hline AT5G61390 & $\begin{array}{l}\text { Polynucleotidyl transferase } \% 2 \mathrm{C} \\
\text { ribonuclease } \mathrm{H} \text {-like superfamily } \\
\text { protein }\end{array}$ & 2,18 & $1,446 \times(10)-06$ & $-1,43$ & 0,0017278 \\
\hline AT1G10690 & cyclin-dependent kinase inhibitor & 1,14 & 0,001876 & $-1,41$ & 0,0001003 \\
\hline AT4G34135 & UDP-glucosyltransferase 73B2 & 1,35 & 0,0025775 & $-1,41$ & 0,0020573 \\
\hline AT1G67970 & heat shock transcription factor $A 8$ & 2,84 & $5,373 \times(10)-20$ & $-1,41$ & $1,698 \times(10)-06$ \\
\hline AT4G12720 & MutT/nudix family protein & 2,57 & 0,0001186 & $-1,41$ & 0,0346772 \\
\hline AT2G31880 & $\begin{array}{l}\text { Leucine-rich repeat protein kinase } \\
\text { family protein }\end{array}$ & 2,70 & $1,358 \times(10)-08$ & $-1,41$ & 0,0042766 \\
\hline AT1G19230 & $\begin{array}{l}\text { Riboflavin synthase-like } \\
\text { superfamily protein }\end{array}$ & 2,00 & 0,0008739 & $-1,40$ & 0,0259286 \\
\hline AT1G11300 & $\begin{array}{l}\text { G-type lectin S-receptor-like } \\
\text { Serine/Threonine-kinase }\end{array}$ & 1,25 & 0,027013 & $-1,40$ & 0,0128349 \\
\hline AT2G15042 & $\begin{array}{l}\text { Leucine-rich repeat (LRR) family } \\
\text { protein }\end{array}$ & 1,07 & 0,0122342 & $-1,39$ & 0,0022716 \\
\hline AT5G61790 & calnexin 1 & 1,83 & $1,027 \times(10)-08$ & $-1,39$ & $4,449 \times(10)-05$ \\
\hline AT3G45640 & mitogen-activated protein kinase 3 & 1,23 & 0,0021048 & $-1,35$ & 0,0006973 \\
\hline AT2G47130 & $\begin{array}{l}\text { NAD(P)-binding Rossmann-fold } \\
\text { superfamily protein }\end{array}$ & 3,63 & $3,358 \times(10)-12$ & $-1,35$ & 0,0125374 \\
\hline AT1G24150 & formin homologue 4 & 2,36 & $4,675 \times(10)-05$ & $-1,35$ & 0,0283148 \\
\hline AT1G66880 & Protein kinase superfamily protein & 1,94 & $5,206 \times(10)-07$ & $-1,35$ & 0,000903 \\
\hline AT2G40095 & $\begin{array}{l}\text { Alpha/beta hydrolase related } \\
\text { protein }\end{array}$ & 2,54 & 0,0001398 & $-1,34$ & 0,0436434 \\
\hline
\end{tabular}




\begin{tabular}{|c|c|c|c|c|c|}
\hline AT4G21400 & $\begin{array}{l}\text { cysteine-rich RLK (RECEPTOR-like } \\
\text { protein kinase) } 28\end{array}$ & 2,18 & $3,865 \times(10)-05$ & $-1,34$ & 0,0129562 \\
\hline AT1G21520 & hypothetical protein & 3,60 & $1,907 \times(10)-08$ & $-1,32$ & 0,0380118 \\
\hline AT5G12930 & inactive rhomboid protein & 1,31 & 0,0205344 & $-1,32$ & 0,0154024 \\
\hline AT1G22070 & transcription factor TGA3 & 1,09 & 0,0004009 & $-1,32$ & $2,761 \times(10)-05$ \\
\hline AT5G57685 & glutamine dumper 3 & 1,08 & 0,0029859 & $-1,30$ & 0,0003104 \\
\hline AT5G13190 & GSH-induced LITAF domain protein & 1,71 & 0,001285 & $-1,30$ & 0,0140636 \\
\hline AT1G65240 & $\begin{array}{l}\text { Eukaryotic aspartyl protease family } \\
\text { protein }\end{array}$ & 2,74 & $1,159 \times(10)-05$ & $-1,30$ & 0,0126986 \\
\hline AT5G64870 & $\begin{array}{l}\text { SPFH/Band 7/PHB domain- } \\
\text { containing membrane-associated } \\
\text { protein family }\end{array}$ & 1,43 & 0,0023682 & $-1,28$ & 0,0073876 \\
\hline AT1G04980 & PDI-like 2-2 & 2,58 & $4,045 \times(10)-14$ & $-1,27$ & 0,0005964 \\
\hline AT4G05590 & pyruvate carrier-like protein & 1,10 & 0,0242346 & $-1,27$ & 0,0087129 \\
\hline AT3G24090 & $\begin{array}{l}\text { putative glucosamine-fructose-6- } \\
\text { phosphate aminotransferase }\end{array}$ & 2,05 & $7,296 \times(10)-08$ & $-1,26$ & 0,0010623 \\
\hline AT5G39030 & Protein kinase superfamily protein & 1,17 & 0,0014495 & $-1,25$ & 0,0008702 \\
\hline AT2G02810 & UDP-galactose transporter 1 & 2,97 & $9,642 \times(10)-14$ & $-1,25$ & 0,0024138 \\
\hline AT3G17420 & glyoxysomal protein kinase 1 & 1,84 & 0,0002184 & $-1,25$ & 0,015095 \\
\hline AT3G52400 & syntaxin of plants 122 & 2,89 & $8,807 \times(10)-07$ & $-1,25$ & 0,0396749 \\
\hline AT3G13380 & BRI1-like 3 & 2,65 & $9,353 \times(10)-09$ & $-1,22$ & 0,0079046 \\
\hline AT3G44900 & cation/H+ exchanger 4 & 1,31 & 0,0126867 & $-1,22$ & 0,0159921 \\
\hline AT1G71110 & transmembrane protein & 1,10 & 0,0009986 & $-1,22$ & 0,0003044 \\
\hline AT1G67000 & Protein kinase superfamily protein & 1,39 & 0,0232616 & $-1,21$ & 0,0397374 \\
\hline AT1G14480 & Ankyrin repeat family protein & 1,46 & $4,263 \times(10)-05$ & $-1,19$ & 0,0011862 \\
\hline AT4G37690 & $\begin{array}{l}\text { Galactosyl transferase } \\
\text { GMA12/MNN10 family protein }\end{array}$ & 1,68 & 0,0016375 & $-1,18$ & 0,0338384 \\
\hline AT1G61470 & $\begin{array}{l}\text { Polynucleotidyl transferase } \% 2 \mathrm{C} \\
\text { ribonuclease } \mathrm{H} \text {-like superfamily } \\
\text { protein }\end{array}$ & 1,35 & 0,0163643 & $-1,18$ & 0,0282807 \\
\hline AT5G42020 & $\begin{array}{l}\text { Heat shock protein } 70 \text { (Hsp 70) } \\
\text { family protein }\end{array}$ & 1,99 & $2,114 \times(10)-08$ & $-1,17$ & 0,0024962 \\
\hline AT1G09210 & calreticulin $1 b$ & 1,33 & $9,24 \times(10)-05$ & $-1,14$ & 0,0016966 \\
\hline AT3G18770 & Autophagy-related protein 13 & 1,09 & 0,0007505 & $-1,14$ & 0,0004709 \\
\hline AT5G21090 & $\begin{array}{l}\text { Leucine-rich repeat (LRR) family } \\
\text { protein }\end{array}$ & 1,16 & 0,0019631 & $-1,13$ & 0,0027894 \\
\hline AT5G45800 & $\begin{array}{l}\text { Leucine-rich repeat protein kinase } \\
\text { family protein }\end{array}$ & 1,21 & $4,479 \times(10)-05$ & $-1,11$ & 0,0002849 \\
\hline AT5G54720 & Ankyrin repeat family protein & 1,27 & 0,0145911 & $-1,11$ & 0,0482934 \\
\hline AT3G60470 & $\begin{array}{l}\text { transmembrane protein\%2C } \\
\text { putative (DUF247) }\end{array}$ & 3,15 & $4,562 \times(10)-07$ & $-1,09$ & 0,0455912 \\
\hline AT3G52480 & transmembrane protein & 1,08 & 0,0183989 & $-1,09$ & 0,0169711 \\
\hline AT3G23280 & hypothetical protein & 1,28 & $8,615 \times(10)-06$ & $-1,08$ & 0,0001456 \\
\hline AT5G12890 & $\begin{array}{l}\text { UDP-Glycosyltransferase } \\
\text { superfamily protein }\end{array}$ & 1,13 & 0,000165 & $-1,08$ & 0,0004635 \\
\hline AT4G22780 & ACT domain repeat 7 & 1,12 & 0,0004499 & $-1,07$ & 0,0009513 \\
\hline AT4G39830 & Cupredoxin superfamily protein & 2,68 & $2,404 \times(10)-08$ & $-1,06$ & 0,0291891 \\
\hline AT1G08450 & calreticulin 3 & 2,29 & $9,076 \times(10)-10$ & $-1,06$ & 0,0074537 \\
\hline AT5G07340 & Calreticulin family protein & 1,63 & $1,653 \times(10)-08$ & $-1,06$ & 0,0004927 \\
\hline AT1G72280 & $\begin{array}{l}\text { endoplasmic reticulum } \\
\text { oxidoreductins } 1\end{array}$ & 2,11 & $6,821 \times(10)-07$ & $-1,05$ & 0,0175291 \\
\hline
\end{tabular}




\begin{tabular}{l|l||r|r|r|r}
\hline AT1G32700 & $\begin{array}{l}\text { PLATZ transcription factor family } \\
\text { protein }\end{array}$ & 1,06 & 0,0038667 & $-1,05$ & 0,0040435 \\
\hline AT5G54710 & Ankyrin repeat family protein & 1,24 & $1,159 \times(10)-05$ & $-1,04$ & 0,0004844 \\
\hline AT3G23110 & receptor like protein 37 & 1,03 & 0,0059882 & $-1,03$ & 0,0074254 \\
\hline AT2G39705 & ROTUNDIFOLIA like 8 & 1,56 & 0,000621 & $-1,03$ & 0,0186671 \\
\hline AT1G77810 & $\begin{array}{l}\text { Galactosyltransferase family } \\
\text { protein }\end{array}$ & 1,87 & $1,72 \times(10)-06$ & $-1,02$ & 0,0105229 \\
\hline AT1G80460 & $\begin{array}{l}\text { Actin-like ATPase superfamily } \\
\text { protein }\end{array}$ & 1,06 & 0,0010917 & $-1,01$ & 0,0023086 \\
\hline AT2G24240 & $\begin{array}{l}\text { BTB/POZ domain with WD40/YVTN } \\
\text { repeat-like protein }\end{array}$ & 1,89 & $7,986 \times(10)-08$ & $-1,00$ & 0,005515 \\
& & &
\end{tabular}




\section{Acknowledgements}

I would like to express my sincere gratitude to all the people who made this possible.

First and foremost, I thank my family. Thank you, Mama and Tata for all the sacrifices you took just so I can be here today. Thank you, Ita and Lucija for believing in me and supporting me throughout my life.

Thank you, Joshua for all the love and support you provided during the last ten years of my life. And thank you for leaving everything and coming with me for a craziest ride so far. I am sure, we will have some more. And thank you, Anić family, for letting him go.

Thank you to all my friends spread around the globe. I never imagined having such a support and love from all of you. Thank you to my building mates and their dearest Aswin, Daniel, Christina, Louisa, Ali, Lisa, Sina, Denise and Pascal. I really love you guys and I would not be able to do this without you. Thank you to my Canadian mates Karen, Krešo, Sean, Yang, Ana, Kevin, Ludo and Woody for babysitting me in Vancouver. I was such a dream, honestly. Thank you to IRTG people Mimi, Jessi, Dimi, Sven, Lara, Milena, Karl, Athanas, Ling and Kisore for the support you provided. Thank you to beautiful people surrounding me outside the lab, Magda and Marinkica, Clemance and Pierra, Jakob and Mareen. Thank you to my friends back home, for being excited for me and living this opportunity with me. Thank you, Tena, Terka, Petra, Mia, Vale, Simke, Susi and Ljiljo. I love you all.

Thank you to all current and former members of the Gatz lab. Thank you, Anna, Kathi and Ronny, for teaching me everything you know and doing it so patiently. Thank you for all the experiments you have done for me without complaining, at least not too much. Thank you, Guido, for all the patience you had with my constant interruptions of your work. I am truly grateful for your ability to find an answer to any question. Thank you, senior researches, Joachim and Irene for your wisdom and advices. Thank you, former members of the lab, Jan, Ning, Li-Jun, Armin, Katrin and my ex-students Jasmin and Dyari. Thank you, Feli for preparing all the soil pots for the last three years. And welcome new students, Anja and Isha. I hope you will like it here.

Finally, I would like to thank two people who dared to take a student completely out of the frame and trusted her to fulfill the task. Corinna, you have been taking care of me since the 
first day I stepped in this building three years ago. Almost everything I know about research and how it should be conducted, I have learnt from you. Thank you for this.

Thank you, professor, for giving me the opportunity to do this. Thank you for the guidance and all your advices.

It has been a bumpy road, but I am glad I took it. 
